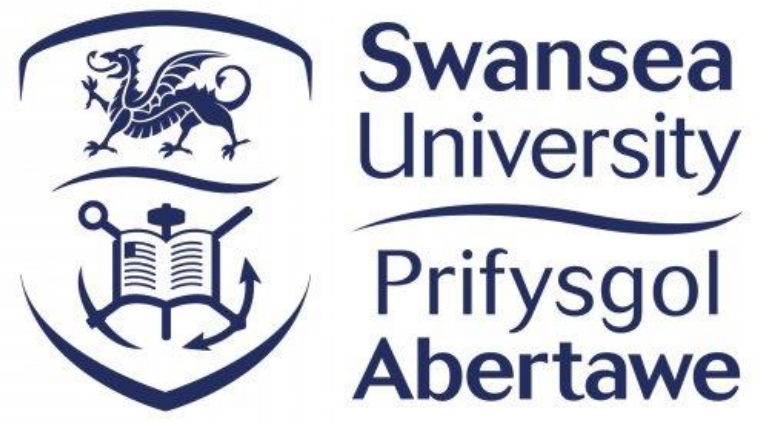

\title{
Harbouring Aliens: Ports as Habitats for Non-native Species
}

\author{
Samuel John Holmes
}

Submitted to Swansea University in fulfilment of the requirements for the Degree of Doctor of Philosophy

\section{Swansea University}

2021 


\section{SUMMMARY}

Non-native species are widely regarded as a significant environmental threat and have been associated with biodiversity loss, species extinctions and the altering of ecosystem services. Shipping is the largest transport vector for aquatic species worldwide and ports, being central to this industry, are considered potential 'hotspots' for non-native species introductions. Ports are often heavily industrialised areas which can hinder access and the use of standard survey methods. As such, our knowledge of non-native species within UK ports is poor. As the foundation for this research I developed two different settlement surveys designed specifically to overcome some of the obstacles associated with working within active port environments. Surveys were deployed across five ports in South Wales, UK. I detected 13 non-native species and described the wider fouling communities present within each studied port. Whole community structure and their succession varied highly between ports, with salinity being the primary driver of differences between port communities. Significant differences in the observed non-native species between ports, independent of geographic proximity, highlighted the need to monitor individual ports with a view to implementing bespoke, effective management strategies. Colonisation of different material substrates was quantified and revealed that whilst the community structure varied between material types, non-native species would readily colonise all studied materials. These findings have particular importance for the management of non-native species within ports and have been used to inform port biosecurity procedures within the studied region. The successful deployment of the tailored survey methods within active ports will encourage regular monitoring for non-native species within UK ports. This research also showed that collaborations between researchers and port authorities can be highly effective for both parties and may indeed be necessary if we are to successfully manage aquatic non-native species within the UK. 


\section{DECLARATION AND STATEMENTS}

DECLARATION

This work has not previously been accepted in substance for any degree and is not being concurrently submitted in candidature for any degree.

Signed ...... (candidate)

Date ......28/10//2020

\section{STATEMENT 1}

This thesis is the result of my own investigations, except where otherwise stated. Where correction services have been used, the extent and nature of the correction is clearly marked in a footnote(s).

Other sources are acknowledged by footnotes giving explicit references. A bibliography is appended.

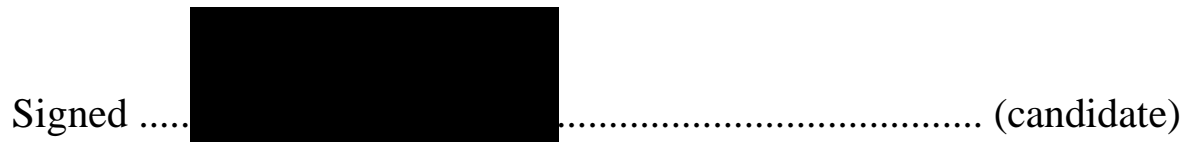

Date .....28/10/2020

\section{STATEMENT 2}

I hereby give consent for my thesis, if accepted, to be available for photocopying and for inter-library loan, and for the title and summary to be made available to outside organisations.

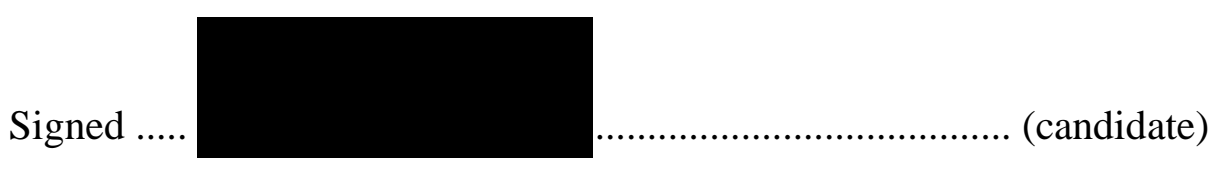

Date 


\section{Authorship Declaration}

The following people and institutions contributed to the publication of work undertaken as part of this thesis:

Candidate Samuel John Holmes (Biosciences)

Author 1 Ruth Callaway (Swansea University)

\section{Author Details and their Roles:}

Paper 1: Design and implementation of two surveys targeted at describing fouling communities and identifying non-native species within active ports (published 2020)

\section{Located in Chapter 1}

Candidate contributed to the conception of the research, survey planning and design, data collection, data analysis, writing

Author 1 contributed to the conception of the research, survey design and reviewing of drafts.

Paper 2: Fouling communities and non-native species within five ports along the Bristol Channel, South Wales, UK (published 2021)

\section{Located in Chapter 2}

Candidate contributed conception of the research, survey planning and design, data collection, data analysis, writing

Author 1 contributed to the conception of the research, survey design and reviewing of drafts. 
We the undersigned agree with the above stated "proportion of work undertaken" for each of the above published peer-reviewed manuscripts contributing to this thesis:

Signed Candidate

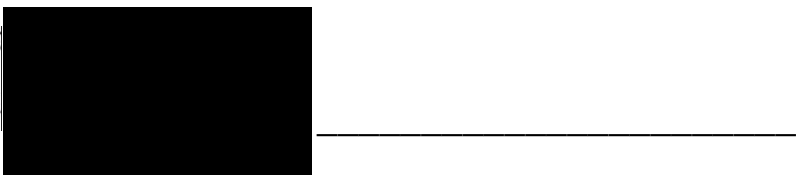

Author 1

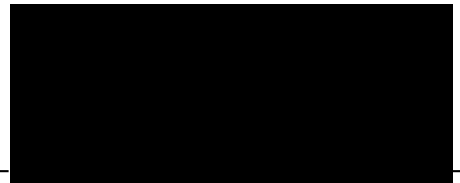

Ethics: Ethical approval for this research was granted by the Swansea University College of Science ethics panel. Approval number: SU-Ethics-Student-040920/3058.

Funding: Funds were secured through the Knowledge Economy Skills Scholarships:

Knowledge Economy Skills Scholarships (KESS 2) is a pan-Wales higher level skills initiative led by Bangor University on behalf of the HE sector in Wales. It is part funded by the Welsh Government's European Social Fund (ESF) convergence programme for West Wales and the Valleys.

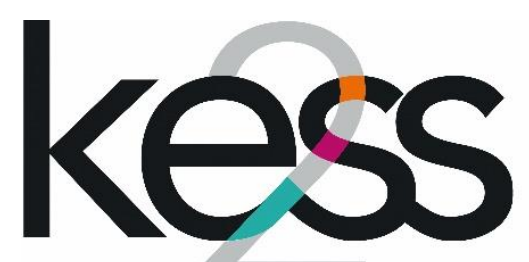

Ysgoloriaethau Sgiliau Economi Gwybodaeth Knowledge Economy Skills Scholarships

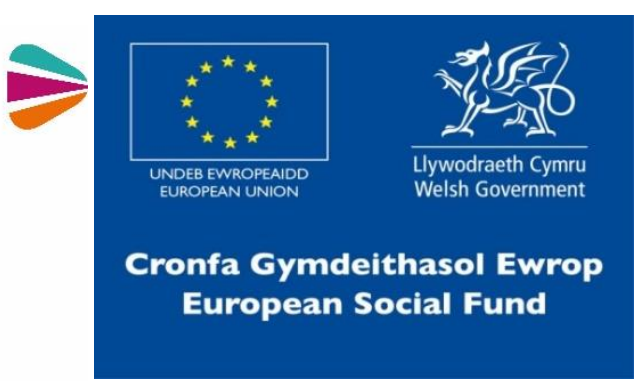




\section{TABLE OF CONTENTS}

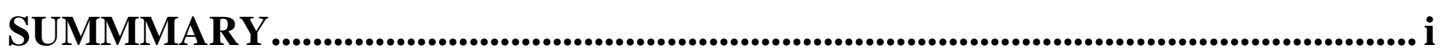

DECLARATION AND STATEMENTS..................................................................... ii

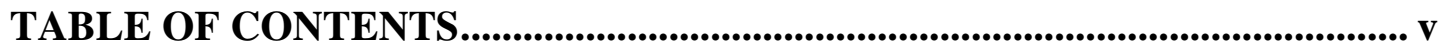

ACKNOWLEDGEMENTS........................................................................... vii

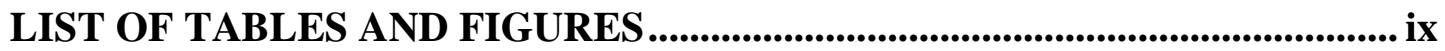

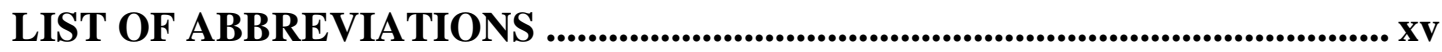

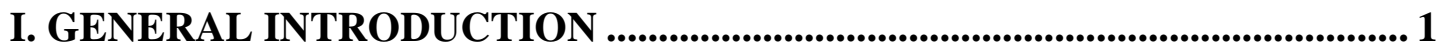

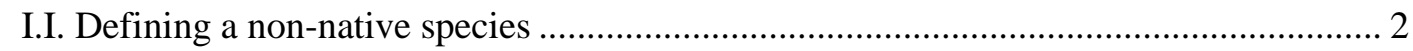

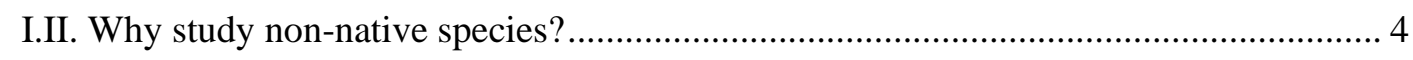

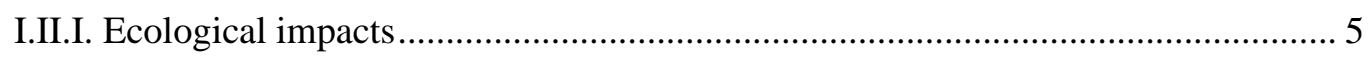

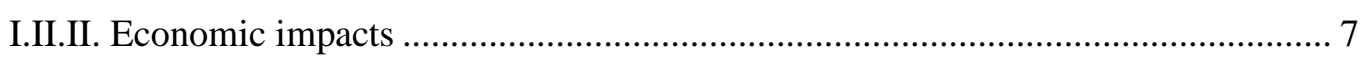

I.II.III. Ecosystem service approach ................................................................... 8

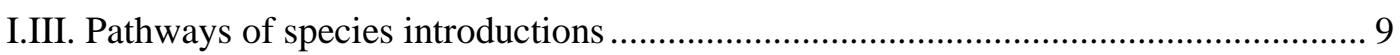

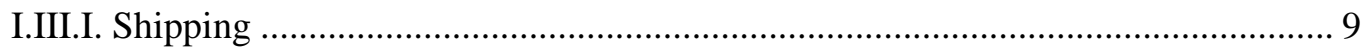

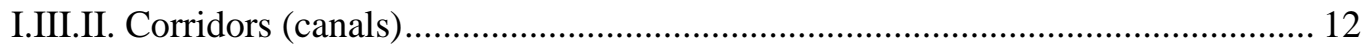

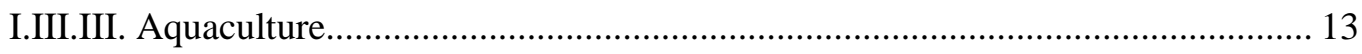

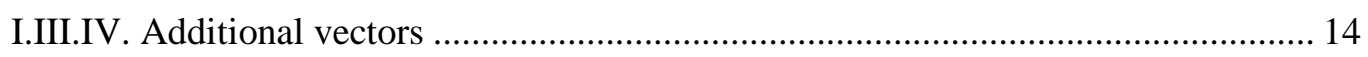

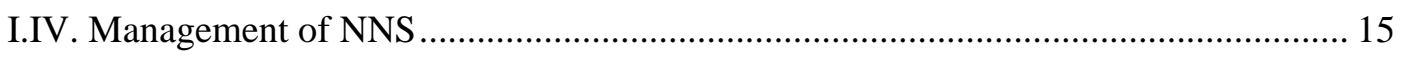

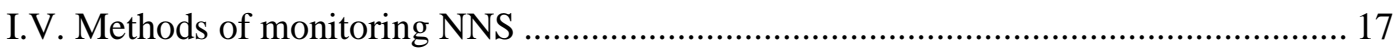

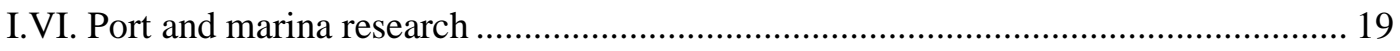

I.VI.I. Quantified literature search into port- and marina-based surveys....................... 20

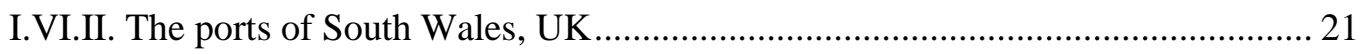

II. THESIS AIMS AND OBJECTIVES....................................................... 25

CHAPTER 1: Design and implementation of two surveys targeted at describing fouling communities and identifying non-native species within active ports* ${ }^{*} . .29$

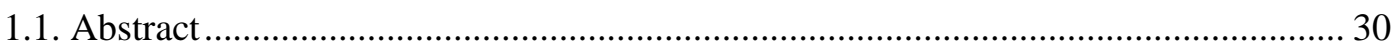

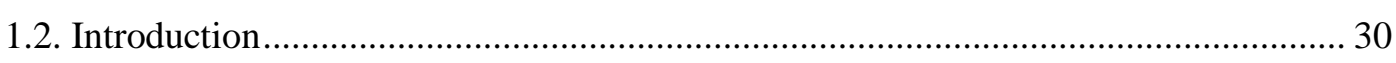

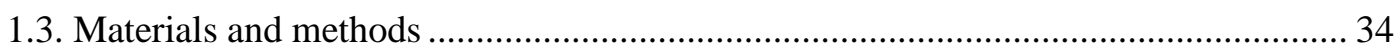




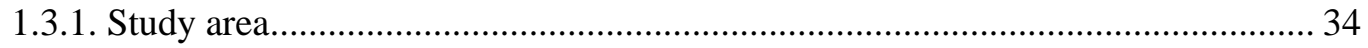

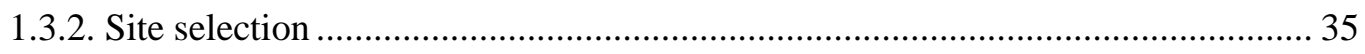

1.3.3. Successional Settlement Survey (SSS) ……....................................................... 36

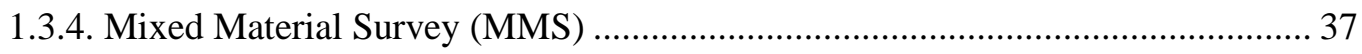

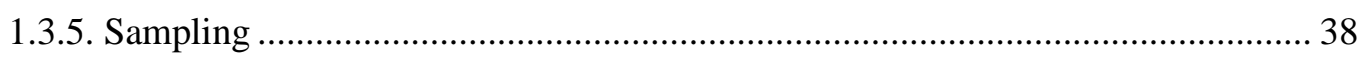

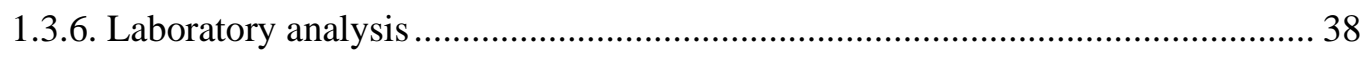

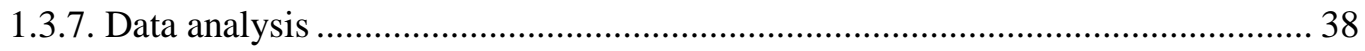

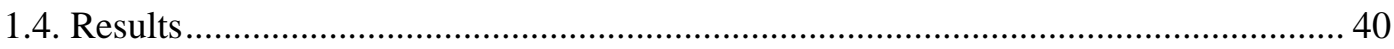

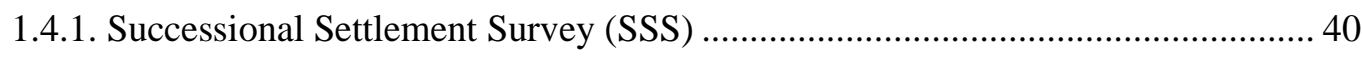

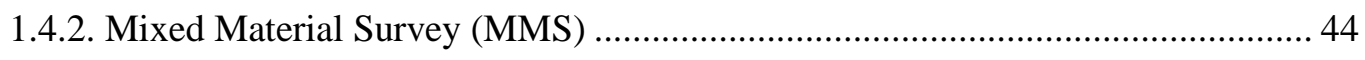

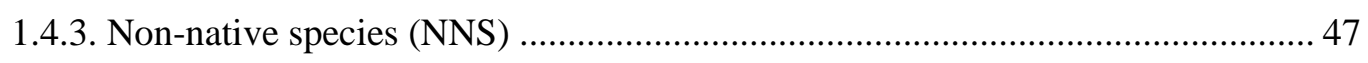

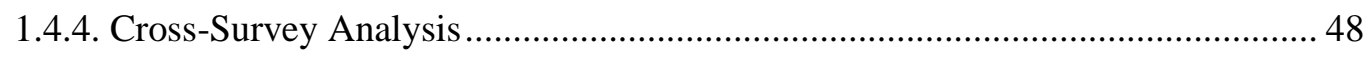

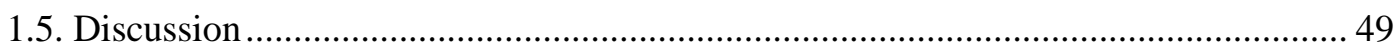

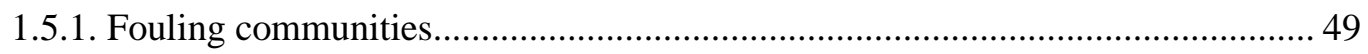

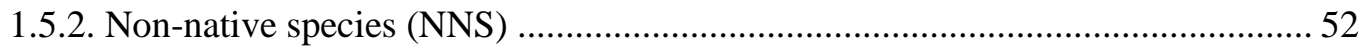

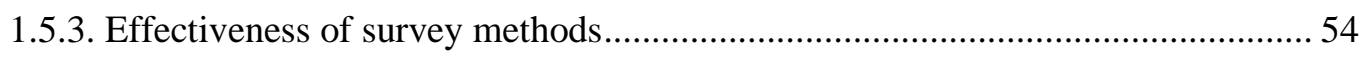

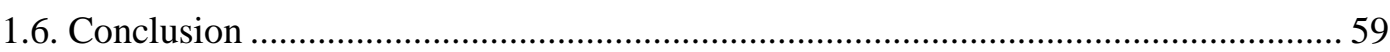

CHAPTER 2: Fouling communities and non-native species within five ports along the Bristol Channel, South Wales, UK ${ }^{*}$................................................... 61

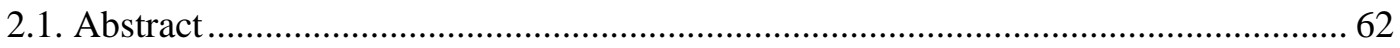

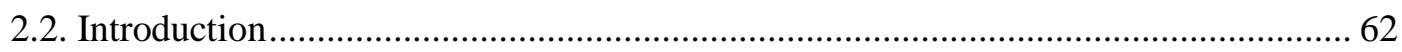

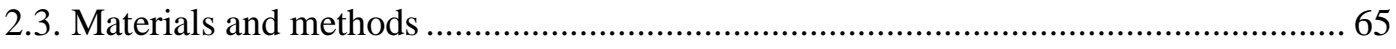

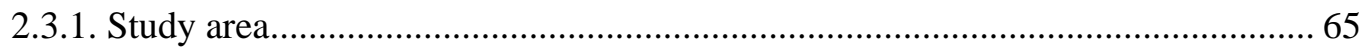

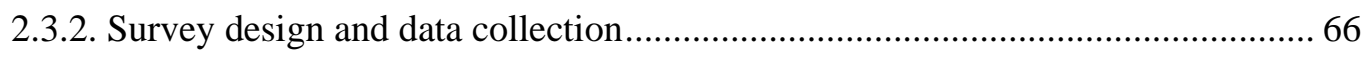

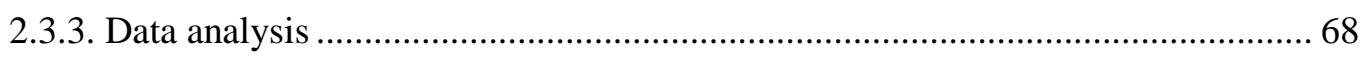

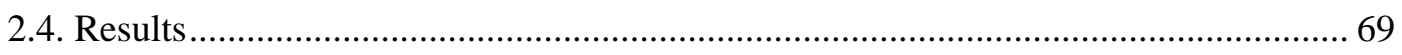

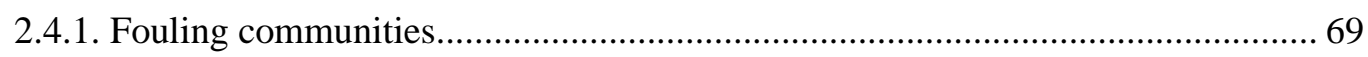

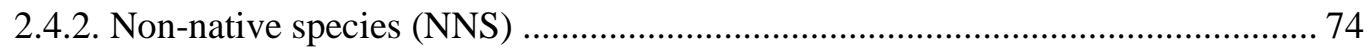

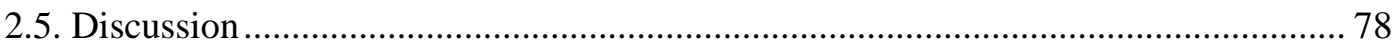

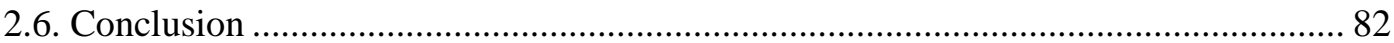

CHAPTER 3: Material selection by fouling non-native species present in port

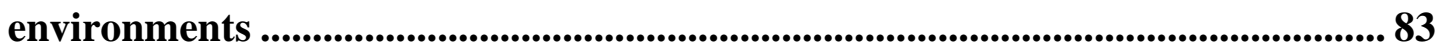

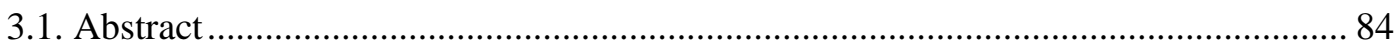

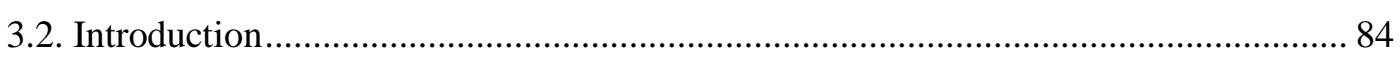

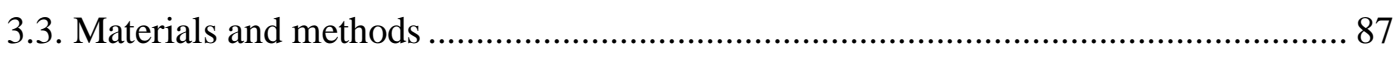

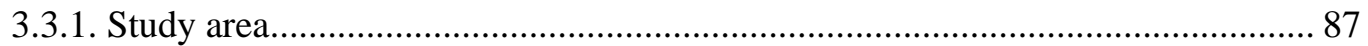


3.3.2. Survey design

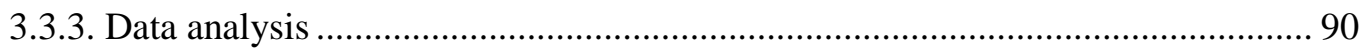

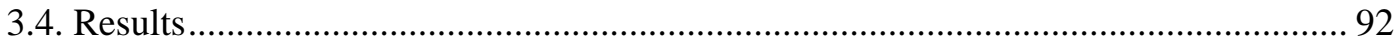

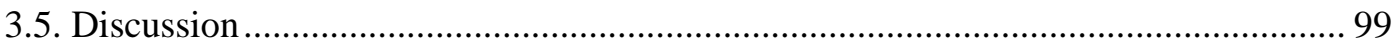

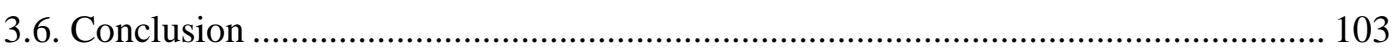

CHAPTER 4: Validating the use of plastic settlement tiles in describing fouling communities and identifying non-native species within active port environments 104

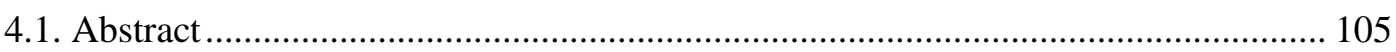

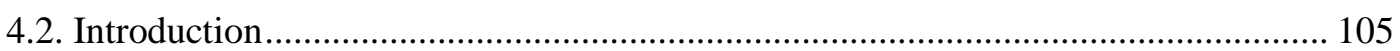

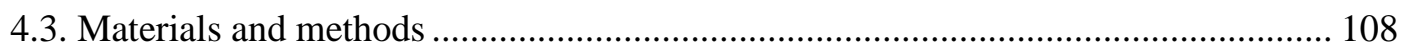

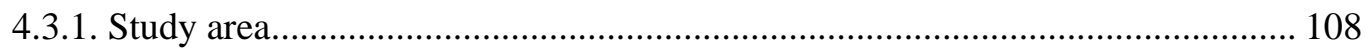

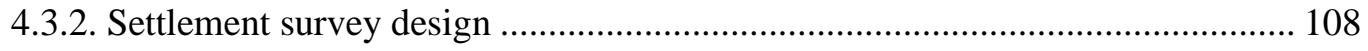

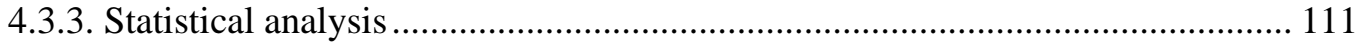

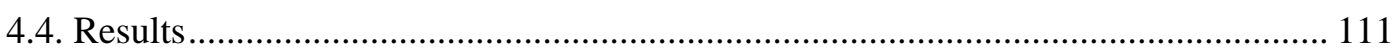

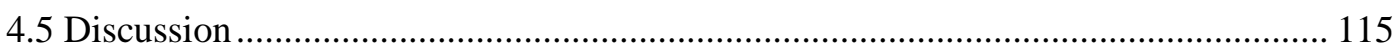

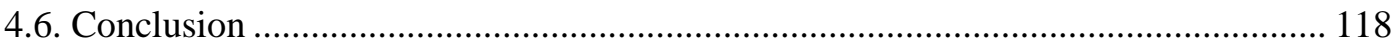

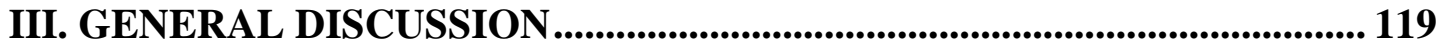

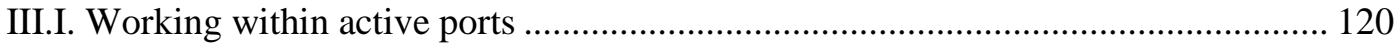

III.I.I. Future direction of port-based surveys ......................................................... 125

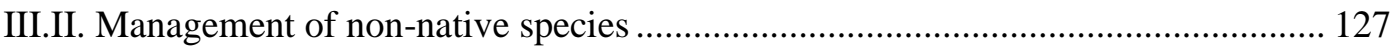

III.III. Non-native species in the wider fouling community .......................................... 130

III.IV. A note on impacts of the Covid-19 pandemic ....................................................... 132

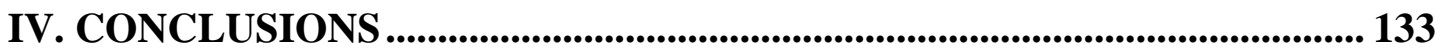

V. APPENDIX I: SUPPLEMENTARY TABLES ................................................ 135

VI. APPENDIX II: ENVIRONMENTAL VARIABLES (CHAPTER 2) ......... 145

VII. APPENDIX III: SAMPLING PHOTOS.............................................. 148

VIII. APPENDIX IV: NON-NATIVE SPECIES FIELD IDENTIFICAITON

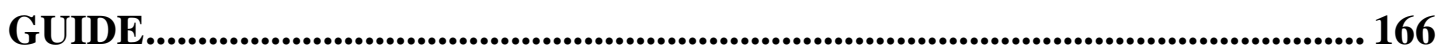

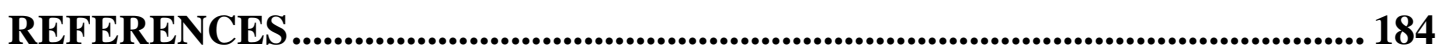




\section{ACKNOWLEDGEMENTS}

I have to start by giving the most incredible amount of thanks to my supervisor, $\mathrm{Dr}$ Ruth Callaway, whose advice, guidance and support have made this $\mathrm{PhD}$ first a possibility and now a reality. Ruth, I have thoroughly enjoyed working with you these last few years and I shall forever be indebted to you for all your help.

I would like to thank Martin Lightwood, Elton Prance and Christine Nelson, all from Associated British Ports, who have given me huge amounts of advice for working within their ports and, ultimately, without whom this research may very well have not been possible. I extend this thanks to all of the GPMO guys at ABP (Matt, Jordan, Gary, and everyone else) for the many, many hours spent helping me in the field. Many thanks to Dr Tom Fairchild and Dr John Griffin for your time, use of the lab and associated expensive equipment, and the snippets of sound advice throughout the $\mathrm{PhD}$. Thanks to everyone who has helped me out in the labs, even if it was just putting up with the occasional bad smells I would bring in with my benthic samples apologies, again. Thanks to Phil Hopkins in the university workshop for helping me in the construction of my survey materials. Thanks to all the fellow $\mathrm{PhD}$ students and general postgrad/ research community, especially those who I was fortunate to share an office with. We had many laughs and the PhD was definitely more enjoyable because of all of you. Similar thanks go to all my friends and family, near and far, who have provided plenty of support and memories away from the $\mathrm{PhD}$ these last few years which has helped me stumble my way to this point.

Mom and dad, thanks so much for your ongoing support throughout but especially these last few months. You have always supported me in my scientific endeavours even without knowing or understanding a great deal about what I get up to. Quite how you managed to read this entire thesis and remain interested is beyond me so thanks very much for the enthusiasm and input.

There are countless people who have helped me a tremendous amount throughout this $\mathrm{PhD}$, be it directly or indirectly, who I have not been able to name here. I would like to throw a blanket 'thank you' over all of you and hope to be able to provide many of you with thankyous in person soon. Thanks all. 


\section{LIST OF TABLES AND FIGURES}

Table 1. Mean abundance of NNS per $500 \mathrm{~cm}^{2}$ ( \pm standard error) of each NNS recorded within the two survey types: Successional Settlement Survey (SSS) and Mixed Material Survey (MMS).

Table 2. Salinity, shipping traffic and species richness of ports at the Bristol Channel (East to West). Salinity range over 11 months (2019), mean shipping traffic calculated from ABP logs; total number of species colonising settlement plates in 2019.

Table 3. Non-native species recorded in South Wales ports. GB establishment status and existing presence in the Bristol Channel based on data from NBN Atlas (NBN Atlas, 2020). * indicates species previously known to inhabit the recorded port(s) but not the Bristol Channel.

Table 4. Salinity and temperature ranges present in each of the five studied ports.

Table 5. Total abundance of non-native species observed within each surveyed port.

Table 6. Pairwise comparisons between total abundance of fauna colonising different materials in ports (Dunn's tests) Bold p-values indicate significant differences at the $95 \%$ significance level.

Table 7. Results of PERMANOVA main and pairwise tests to determine the effect of material type on whole community composition and total abundance of organisms within the ports of Barry and Swansea. Only material pairs that yielded a significant $\mathrm{p}$ value are listed. Significant $\mathrm{p}$ values $(\mathrm{p}<0.05)$ are highlighted in bold. $\mathrm{p}$ values for main tests $=$ permutation $\mathrm{p}$, for pairwise tests $=$ Monte Carlo $\mathrm{p}$.

Table 8. Output data from a one-way SIMPER analysis with the factor 'material type'. Analysis conducted using $\log (\mathrm{X}+1)$ transformed community abundance data for two material types: plastic and slate. Plastic: $n=12$. Slate: $n=10$. 
Supplementary Table S1. Total abundance/ area covered $\left(\mathrm{cm}^{2}\right)$ of species within each survey month, identified as part of the Successional Settlement Survey. Area covered is denoted by a ${ }^{* * \star}$, all other numbers are abundance values. Species are grouped within Phylum. NNS denoted by a ‘ ${ }^{\dagger}$.

Supplementary Table S2. Total abundance and area covered $\left(\mathrm{cm}^{2}\right)$ of species identified from materials forming the Mixed Material Survey. Area covered is denoted by '*', all other values are abundance counts. NNS denoted by a '†'.

Supplementary Table S3. Abundance count or measure of coverage $\left(\mathrm{cm}^{2}\right)$ for each species recorded within each of the five studied ports: Newport, Cardiff, Barry, Port Talbot, Swansea. * denotes species recorded as area covered when abundance counts were not possible. ${ }^{\dagger}$ denotes non-native species.

Supplementary Table S4. Abundance count or measure of coverage $\left(\mathrm{cm}^{2}\right)$ for each species recorded within each of the five studied ports: Newport, Cardiff, Barry, Port Talbot, Swansea. * denotes species recorded as area covered when abundance counts were not possible. ${ }^{\dagger}$ denotes non-native species.

Supplementary Table S5. Non-native species observed colonising different material types in each of the five studied ports. Port denoted by letter: Newport $=\mathrm{N}$, Cardiff $=$ C, Barry = B, Swansea $=$ S. No non-native species observed from Port Talbot.

Supplementary Table S6. Abundance count or measure of coverage $\left(\mathrm{cm}^{2}\right)$ for each species recorded colonising plastic and slate settlement tiles. * denotes species recorded as area covered when abundance counts were not possible. ${ }^{\dagger}$ denotes nonnative species.

Figure 1. Adapted and simplified from Blackburn et al. (2011); 'A proposed unified framework for biological invasions'. The invasion process is broken down into stages (green) with species having to overcome necessary barriers (blue) to progress along the invasion pathway. Different terminology (red) is highlighted for different stages of the invasion process.

Figure 2. Schematic representation of the search criteria and selection process involved in finding literature relevant to the literature search described in section I.VI.I. 
Figure 3. Location of five Associated British Ports within South Wales.

Figure 4. Map of each of the ports of Newport, Cardiff, Barry, Port Talbot and Swansea showing the position of individual docks within each port and the position relative to either the Bristol Channel or Severn Estuary.

Figure 5. Map outlining the position of survey sites within the Port of Swansea. Each zone contains a location for the deployment of both a 'Successional Settlement Survey' and a 'Mixed Material Survey'. Zones are outlined showing paired sites.

Figure 6. Successional Settlement Survey materials prior to deployment.

Figure 7. Mixed Material Survey materials prior to deployment.

Figure 8. nMDS plot of colonising species communities collected within the Successional Settlement Survey. Plot based on a resemblance matrix created using a Bray Curtis similarity indices of $\log (\mathrm{X}+1)$ transformed abundance data. Samples labelled by factor 'Month'; symbols represent location factor 'Zone'; June samples were removed from plot since communities were so species-poor that they could not be plotted in a meaningful way in relation to subsequent months.

Figure 9. Colonisation of settlement tiles in Swansea Port $\left(450 \mathrm{~cm}^{2}, \mathrm{n}=3\right)$. A: total number of species recorded. B: number of non-native species (NNS). C: abundance count of Ascidiella scabra. D: surface area coverage $\left(\mathrm{cm}^{2}\right)$ of colonial ascidians. E: abundance count of amphipods. F: abundance count of Aurelia aurita polyps.

Figure 10. Percentage cover of PMMA tiles recorded each month within the Successional Settlement Survey $\left(225 \mathrm{~cm}^{2}, \mathrm{n}=3\right)$. 'Front' refers to the orientation of tiles facing away from port walls, 'Rear' refers to the orientation of tiles facing the port walls.

Figure 11. nMDS plot of samples collected within the MMS. Raw data transformed using a $\log (\mathrm{X}+1)$ transformation. Plot based on a resemblance matrix created using a Bray Curtis similarity index. Samples labelled by factor 'Zone'; symbols represent factor 'Material'.

Figure 12. Percentage cover of organisms present on selected materials from within the MMS after eight months. $\mathrm{n}=3$ for each material. 
Figure 13. Total abundance of organisms per $500 \mathrm{~cm}^{2}$ recorded on each material used within the MMS. Abundance counts standardised using the surface area of each material to allow for direct comparison. $\mathrm{n}=3$.

Figure 14. Average species richness recorded with the two survey types 'successional settlement survey' (SSS) and 'mixed material survey' (MMS). Species per $2700 \mathrm{~cm}^{2}$ for SSS (sum of 6 tiles per zone), $13533 \mathrm{~cm}^{2}$ for MMS (sum of all materials per zone); $\mathrm{n}=3$.

Figure 15. Location and layout of the five ports surveyed as part of this research. Charts of ports by Associated British Ports. https://www.abports.co.uk/

Figure 16. Survey materials prior to deployment.

Figure 17. nMDS plot of fouling community samples collected within 5 ports at bimonthly intervals throughout 2019. Raw data averaged for each sample point ( $\mathrm{n}=$ 2-3) and transformed using a $\log (\mathrm{X}+1)$ transformation. Plot based on a resemblance matrix created using a Bray Curtis similarity index. Samples with no species were removed.

Figure 18. Colonisation of settlement plates at five ports over 10 months. Records per sampling month in the ports of Newport, Cardiff, Barry, Port Talbot and Swansea. A: Mean number of species $450 \mathrm{~cm}^{-2}$. B: mean square root abundance per sample. C: Mean percentage cover per sample. Error bars show standard deviation. $\mathrm{n}$ $=3$ with the exception of Newport (Sep, Oct), Cardiff (Oct), Port Talbot and Swansea (Jul, Sep, Oct), where $n=2$. Abundance based on countable organisms only, percentage cover based on all organisms.

Figure 19. Distance-based redundancy analysis (dbRDA) exploring the amount of variation between communities that may be explained by six abiotic variables (salinity, temperature, $\mathrm{pH}, \mathrm{DO}, \mathrm{ORP}$, ship traffic). Analysis completed using a similarity matrix based on a Bray-Curtis similarity index and square root transformed abundance data. 
Figure 20. Spatial and temporal distribution of the three most abundant non-native species. A: Amphibalanus improvisus as recorded in the ports of Newport and Cardiff. B: Bugulina stolonifera as recorded in the port of Swansea. C: Ficopomatus enigmaticus as recorded in the port of Cardiff. B. stolonifera and F. enigmaticus were each present in only one sample from within the ports of Barry and Newport, respectively, and these data were omitted from the figure.

Figure 21. Location and layout of the five ports surveyed as part of this research. Charts of ports by Associated British Ports. https://www.abports.co.uk/

Figure 22. a: Survey materials prior to deployment. b: schematic list of materials in sequence.

Figure 23. Square root abundance of all recorded organisms per $500 \mathrm{~cm}^{2}$ of surface area for each material group. Data pooled across all ports. $n=12$.

Figure 24. Square root abundance of non-native species per $500 \mathrm{~cm}^{2}$ of surface area for each material group. Data pooled across all ports. $n=12$.

Figure 25. nMDS plot of community samples recorded on 10 different material types within four surveyed ports. Plot based on a similarity matrix created using BrayCurtis index of square root transformed abundance/ coverage data for each sample. Samples labelled by factor 'Material': B = Brick, M = Mop, PMMA (S) = Sanded PMMA, PMMA $($ US $)=$ Unsanded PMMA, PR = Polypropylene rope, PVC $(\mathrm{S})=$ Sanded PVC, PVC $($ US $)=$ Unsanded PVC, $S R=$ Sisal rope, $S=$ Steel, $\mathrm{W}=$ Wood Symbols represent factor 'Port'. Port Talbot samples removed as they were so species-poor they could not be plotted in a meaningful way.

Figure 26. Square root abundance \pm sd of non-natives species (NNS) recorded on each material type in each of four ports: Newport, Cardiff, Barry, and Swansea. No NNS were recorded in the Port of Port Talbot. $n=3$ (Barry, Swansea), $n=2$ (Newport), $\mathrm{n}=1$ (Cardiff).

Figure 27. Location of sites for the deployment of survey materials (slate and plastic) within the Port of Swansea.

Figure 28. Survey materials prior to deployment. a: PMMA tiles, b: Slate tiles Figure 29. Camera rig used to record images of port wall fouling communities 
Figure 30. nMDS plot of fouling community samples observed on two material types and at two sampling sites. Data labels indicate sampling site (site 1 or site 2), symbols indicate material type (plastic or slate). Plot based on a resemblance matrix created using a Bray-Curtis similarity index and $\log (\mathrm{X}+1)$ transformed abundance count data.

Figure 31. a: Mean number of species $450 \mathrm{~cm}^{-2}$ and $\mathrm{b}$ : mean abundance of organisms $450 \mathrm{~cm}^{-2}$ recorded colonising plastic and slate settlement tiles. Data presented for all documented countable species and for non-native species (NNS) only. Error bars show standard deviation. Plastic: $\mathrm{n}=12$. Slate: $\mathrm{n}=10$. '*' denote a significant difference in the colonisation of plastic and slate (Wilcox tests, $\mathrm{p}<0.05$ ).

Figure 32. Still images extracted from video footage of port walls. 


\section{LIST OF ABBREVIATIONS}

\begin{tabular}{|c|c|}
\hline $\mathrm{ABP}$ & Associated British Ports \\
\hline ALWC & Accelerated Low Water Corrosion \\
\hline BWC & Ballast Water Convention \\
\hline $\mathrm{cm}$ & Centimetres \\
\hline $\mathrm{dbRDA}$ & Distance-based Redundancy Analysis \\
\hline DEFRA & Department for Environment, Food \& Rural Affairs \\
\hline DistLM & Distance-based Linear Model \\
\hline DNA & Deoxyribonucleic acid \\
\hline DO & Dissolved Oxygen \\
\hline eDNA & Environmental DNA \\
\hline EEA & European Ecosystem Assessment \\
\hline EU & European Union \\
\hline HELCOM & $\begin{array}{l}\text { Baltic Marine Environment Protection Commission - } \\
\text { Helsinki Commission }\end{array}$ \\
\hline IMO & International Maritime Organisation \\
\hline $\mathrm{L}$ & Litres \\
\hline $\mathrm{m}$ & Metres \\
\hline $\mathrm{mg}$ & Milligrams \\
\hline mi & Miles \\
\hline $\mathrm{mm}$ & Millimetres \\
\hline MMS & Mixed Material Survey \\
\hline $\mathrm{NBN}$ & National Biodiversity Network \\
\hline NGS & Next Generation Sequencing \\
\hline nMDS & Non-metric Multidimensional Scaling \\
\hline NNS & Non-native Species \\
\hline ORP & Oxidation Reduction Potential \\
\hline OSPAR & $\begin{array}{l}\text { A cross government collaboration following the Oslo } \\
\text { and } \underline{\text { Paris conventions }}\end{array}$ \\
\hline PBS & Port Baseline Survey \\
\hline
\end{tabular}


PMMA

PVC

RAS

ROV

SSS

UK

UN

UNCED

UNEP
Poly(methyl methacrylate)

Polyvinyl Chloride

Rapid Assessment Survey

Remotely Operated Vehicle

Successional Settlement Survey

United Kingdom

United Nations

United Nations Conference on Environment and

Development

United Nations Environment Programme 


\section{GENERAL INTRODUCTION}




\section{I.I. Defining a non-native species}

A non-native species (NNS) can be considered as a species 'whose presence within a region is attributable to human actions that enabled them to overcome fundamental biogeographical barriers' (Richardson et al., 2011, Blackburn et al., 2014). Taking the marine environment as an example, a brackish water species may be transported via human actions from a native brackish water environment through a fully marine environment, which the species would otherwise have been unable to overcome, and into a non-native brackish water environment which it can successfully inhabit. Invasion biology is a field of science in which the correct use of terminology, and importantly the clear definition of terms, is crucial to the way we understand and report ecological phenomena.

Non-native, non-indigenous, invasive, alien, introduced, established: these are just a few, and by no means all, of the most frequently used terms in invasion biology to describe the presence and spread of species within locations outside of their natural ranges (e.g. Eno et al., 1997; Ruiz et al., 1997; Bax et al., 2003; Molnar et al., 2008; Dupont et al., 2010; Katsenevakis et al., 2014; Bishop et al., 2015; Booy et al., 2017). Of the terms listed above, 'non-native', 'non-indigenous', and 'alien' can be considered synonymous and are the broadest classification for a species which is found outside of its natural range, following the definition quoted in the opening sentence of this section (Richardson et al., 2011, Blackburn et al., 2014). 'Introduced', 'established', and 'invasive' are terms which each independently describe a species which is in a specific stage of the invasion process (Figure 1; from Blackburn et al., 2011). An introduced species is in the introduction phase of invasion, following an introduction to novel location outside of its native range but has not yet developed a reproducing population (Blackburn et al., 2011). An established species has been able to survive within a novel location and develop a self-sustained reproducing population (Blackburn et al., 2011). An invasive species is a non-native species which has been able to spread beyond the location of initial introduction and invade new locations (Blackburn et al., 2011). That is to say then that all introduced, established and invasive species are non-native species, but not all non-native species are necessarily established or invasive. 
'Invasive' is a term associated with particularly concerning species and is used regularly in policy regulations and legislature to focus on the species which have the highest potential to cause significant impacts (e.g. Molnar et al., 2008; Kettunen et al., 2009; UK Government, 2015; Gallardo et al., 2016; Welsh Government, 2017).

Attempts to outline clear definitions for terms, and to avoid the use of synonymous terms, have been published over the last 15 or so years (Colautti and MacIsaac, 2004; Blackburn et al., 2011, 2014; Richardson et al., 2011). Blackburn et al. (2011) developed arguably the most approachable framework (Figure 1), accessible and relatable to invasion biologists in different fields, to unify the way in which species introduction and invasion were reported. Following a unified framework would enable more accurate reporting, monitoring, and tracking of species dispersal (Blackburn et al., 2011).

Clearly defined terminology does not however overcome the debate surrounding species dispersals which have more complex causes that include the indirect influence of human activity. For example, species range shifts facilitated by climate change do not directly rely on human actions, such as shipping or aquaculture, but arguably may not have occurred if not for the longer-term actions of humans which has driven climate change. I feel the definitions approach of Sorte et al. (2010) to be the most appropriate, whereby species introductions and range shifts can be clearly separated by following the notion that introductions must be facilitated directly by human actions.

Literature cited here was sourced using searches incorporating synonymous terms so as to capture the full spectrum of published literature. There will also be a tendency to focus on aquatic non-native species as opposed to terrestrial ones, based on the nature of the research conducted within this thesis. For this chapter and indeed the entire thesis I have chosen to use the term non-native species (NNS), which is the commonly used term by researchers based in the UK. 


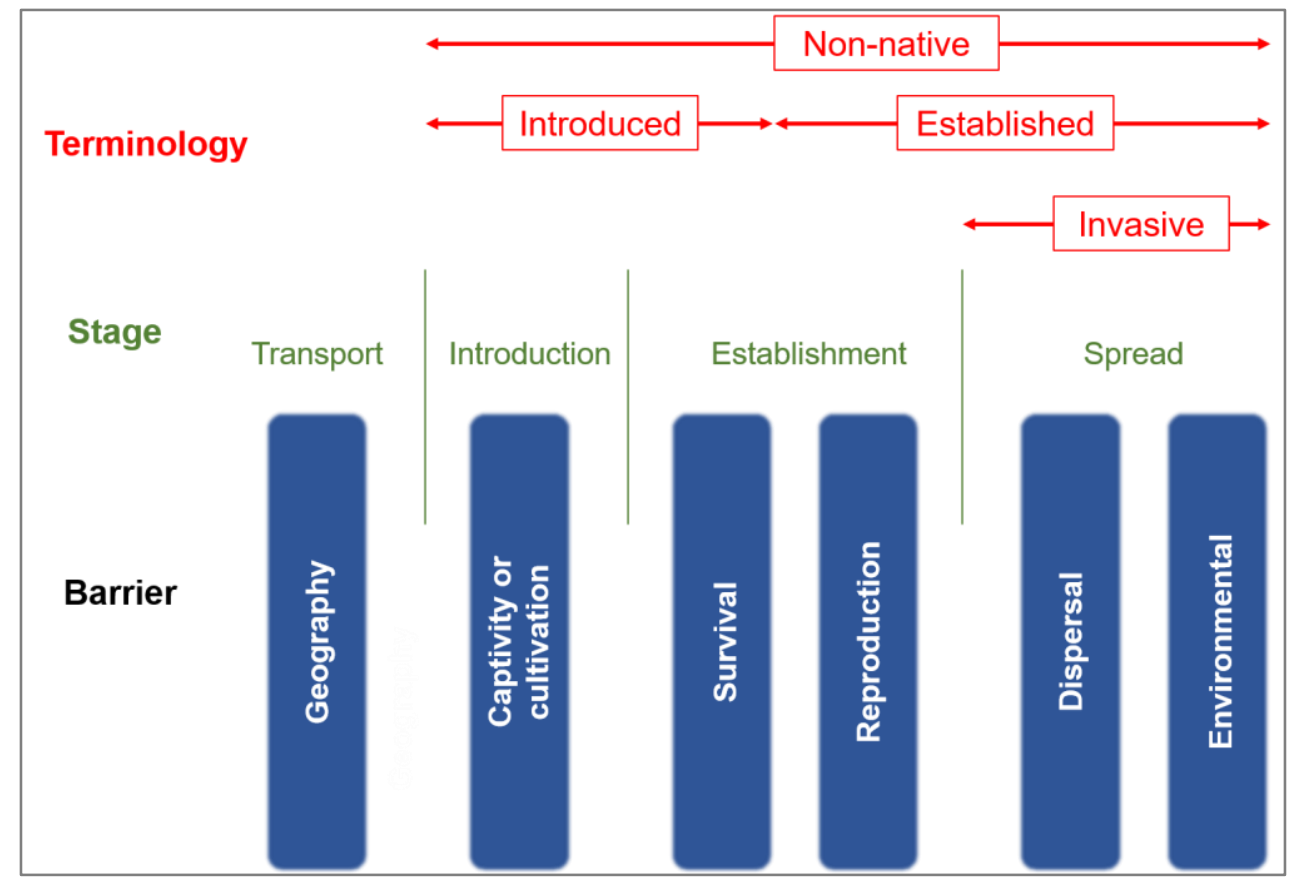

Figure 1. Adapted and simplified from Blackburn et al. (2011); 'A proposed unified framework for biological invasions'. The invasion process is broken down into stages (green) with species having to overcome necessary barriers (blue) to progress along the invasion pathway. Different terminology (red) is highlighted for different stages of the invasion process.

\section{I.II. Why study non-native species?}

In a time where the spotlight on human mediated impacts in the natural world has arguably never been brighter, the importance of monitoring the dispersal of species beyond their native ranges is becoming increasingly important from an ecological, economic, and political point of view. High-profile invasions include the 'Killer Algae', Caulerpa taxifolia, in the Mediterranean (Meinesz and Hesse, 1991; Boudouresque and Verlaque, 2002), the European shore crab, Carcinus maenas, in North America (Roman, 2006) and the rapid invasion of the Black Sea by Mnemiopsis leidyi (Shiganova, 1998). These examples have become such highprofile cases primarily for the scale of impacts towards both human activity and on the environment, as well as the fact that these have all been well studied examples of successful invasions having been identified as NNS early in the invasion process. 


\section{I.II.I. Ecological impacts}

Ecologically, the process of species dispersal and survival within non-native communities has been of interest for many years (Ruiz et al., 1997, 2000; Fridley et al., 2007). As more and more research has been conducted, with more species introductions carefully studied as case studies, there has been a wealth of information published describing both large-scale impacts (for example the loss of biodiversity; Bax et al., 2003) and small-scale impacts (for example species specific competition; Shucksmith et al., 2009) of NNS on natural, native, environments.

Biodiversity loss is a primary concern, with NNS having been suggested to be one of the biggest threats facing biodiversity loss worldwide (Sala et al., 2000; Bax et al., 2003; Molnar et al., 2008; McGeoch et al., 2010). Many of the benefits we gain from the environment, be it for food, culture or for climate regulation, are enhanced with increased biodiversity (UN, 1992). The preservation and prevention of loss of natural biodiversity has therefore been a key principle for a long time in environmental policy and research alike (UN, 1992; UNEP, 2011). Biodiversity policy has continually been shifting and improving goals and targets over time based on an increased evidence base documenting the scale of biodiversity loss and the importance of biodiversity, and NNS are often highlighted as a cause for concern (Sala et al., 2000; Molnar et al., 2008). The main driver of biodiversity loss by NNS is through local species extinctions (Bax et al., 2003; Molnar et al., 2008; McGeoch et al., 2010). McGeoch et al. (2010) provided perhaps the most detailed analysis into the impact of NNS on the loss of species and subsequently biodiversity, whereby they found that NNS 'pressure is driving declines in biodiversity, with all indications that the overall impact on species is increasing'. A more recent review of species extinctions dating back to AD 1500 suggested that NNS are the 'second most common threat associated with species that have gone completely extinct' from five major taxa: plants, amphibians, reptiles, birds, and mammals (Bellard et al., 2016). Ultimately though, the larger-scale impacts of species extinctions and biodiversity loss stem from the accumulation of smaller-scale impacts on native habitats and species. 
Smaller-scale impacts are often reported and discussed in reference to specific species rather than the 'bigger picture' approach when discussing biodiversity loss and extinctions (e.g. Folino-Rorem and Indelicato, 2005; Shucksmith et al., 2009; Charles et al., 2018). Impacts are wide ranging and vary depending on the type of species and the environment into which it is introduced, with impacts generally becoming more severe as the density of the NNS increases (Herbert et al., 2016). NNS have been associated with the loss of higher trophic groups and shifts in food webs to become dominated by lower order consumers (Byrnes et al., 2007). They are known to modify and engineer habitats (Crooks, 2009; Heiman and Micheli, 2010), can directly compete with native species for resources (Shucksmith et al., 2009), and they serve as vectors for disease (Peeler et al., 2011). Most ecological impacts of NNS are associated with established species (Gallardo et al., 2016), however NNS can also cause significant changes to an environment before they become an established species (Ricciardi and Cohen, 2007; Ricciardi et al., 2013).

Whilst much of the focus of the ecological impacts of NNS is towards negative impacts, there is a wealth of research describing the positive impacts that NNS can have on the environment (Wonham et al., 2005; Goodenough, 2010; Schlaepfer et al., 2011; Katsanevakis et al., 2014). An example is the ecosystem engineering Pacific oyster (Magallana gigas). Whilst the displacement of important native species and habitats such as Sabellaria reefs is a clearly negative impact, there is evidence to suggest that $M$. gigas can promote an increase in abundance and diversity of native sessile invertebrates by providing a surface for larval settlement (Padilla, 2010; Herbert et al., 2016). NNS colonisation in low diversity areas has also been found to reduce the recruitment of further NNS, therefore making them more resistant to invasions. However, this is also true for the recruitment of native species (Marraffini and Geller, 2015). Whilst there are well documented positive impacts of NNS, the overriding opinion from both research scientists and policy makers alike remains that NNS should be carefully managed and where possible eradicated from introduced locations (Blackburn et al., 2011; Giakoumi et al., 2019). The risk of negative and irreversible impacts of NNS on the native environment outweighs the potential for positive changes. 
A major problem when attempting to report and document the ecological impacts of NNS is the lack of consistency in quantification (Jeschke et al., 2014). This is often based on the different approaches that researchers take to define impacts as well as the differences in the primary focus of research, for example whether the focus is on the impact to human values or solely ecological (Hulme et al., 2013; Jeschke et al., 2014). The use of ecosystem services to help define ecological impacts is becoming increasingly popular, as this offers a more standardised approach (section I.II.III.; Liquete et al., 2013; Katsanevakis et al., 2014).

\section{I.II.II. Economic impacts}

A number of efforts to quantify the economic impacts of NNS have been published over the last 20 years (Pimentel et al., 2001; Lovell and Stone, 2005; Williams et al., 2010). Reviews generally focus on a national scale, or, if international, within groups of associated countries for example the European Union (e.g. Kettunen et al., 2009). Estimations of the cost of NNS can be highly varied depending on the industries considered in each analysis, with some analyses covering a wide range of industries from tourism to aquaculture whilst others focus on only one sector (e.g. Schultz et al., 2011; Fitridge et al., 2012). To give an example of the potential scale of NNS impacts on an economy, a complete review of the cost of invasive NNS to the British economy has estimated annual losses of $£ 1.7$ billion (Williams et al., 2010).

There have also been efforts to quantify the economic losses caused by specific NNS within a country or region. The European green crab, Carcinus maenas, which is an invasive NNS in North America has been attributed to cause losses of \$18.6 - \$22.6 million per year associated with commercial and recreational shellfisheries and eelgrass restoration efforts on the East coast of the USA (Abt Associates, 2008). Abt associates (2008) also make note that there are likely wider impacts of this species on a number of other fisheries, although these were not quantified. Another well documented impact of a NNS on fisheries is the comb jelly Mnemiopsis ledyi in the Black Sea. 
This species was able to rapidly increase in abundance following an accidental introduction, thought to be from shipping, and ultimately led to the near collapse of anchovy fisheries within the Black Sea causing significant economic losses (Shiganova, 1998).

A major factor contributing to economic losses with respect to aquatic NNS is biofouling. Biofouling organisms can have wide ranging and significant impacts on marine, coastal, and freshwater industry, including maritime trade and fisheries and aquaculture (Floerl and Coutts, 2009; Fitridge et al., 2012). Owing to the large financial implication of this problem there has been a considerable amount of published research either documenting incidences of biofouling or attempting to find effective solutions (Parr et al., 1998; Floerl and Coutts, 2009; Coutts et al., 2010; Callow and Callow, 2011; Ram et al., 2011; McKenzie et al., 2016).

\section{I.II.III. Ecosystem service approach}

Since the publishing of the Millennium Ecosystem Assessment in 2005 (Millennium Ecosystem Assessment, 2005) and a more specific review of marine ecosystem services in 2013 (Liquete et al., 2013), a number of published studies on the impacts of NNS have focussed on how NNS alter ecosystem services (Schlaepfer et al., 2011; Katsanevakis et al., 2014; Vilà and Hulme, 2017). This has encouraged researchers to consider potential impacts across a range of sectors, many of which are of more interest to national and international organisations than purely ecological impacts (for example impacts on human health and the economics of food provision). As we have seen, impacts of NNS introductions can be highly varied (Herbert et al., 2016) and can be both positive and negative (Katsanevakis et al., 2014). Ecosystem services are also being applied in horizon scanning assessments, whereby future potential threats are considered and predicted to improve management and limit the overall impact (Roy et al., 2014). Roy et al. (2018) outline a robust framework for the risk assessment of biological invasions in which the assessment of impacts on ecosystem services is considered one of a number of minimum standards to meet. 
The use of ecosystem services has assisted researchers in bridging the gap between research and policy, whereby impacts of NNS on ecosystem services can be more easily described and presented to policy makers covering a whole range of impacts and therefore presenting the bigger picture. In turn, many governmental strategies and legislation surrounding NNS now focus on addressing the impacts on NNS on ecosystem services (DEFRA, 2011; EU, 2014).

\section{I.III. Pathways of species introductions}

Aquatic species are transported and introduced globally into environments outside of their natural range along several different pathways. Shipping, corridors (e.g. the Suez Canal), and aquaculture are considered the primary vectors for species introductions worldwide, and will therefore take the focus of this section (Carlton and Geller, 1993; Ruiz et al., 1997; Naylor et al., 2001; Molnar et al., 2008; Katsanevakis et al., 2013; Seebens et al., 2013).

\section{I.III.I. Shipping}

Shipping is the most commonly reported source of species introductions and can transport species either within ballast water or through biofouling of species settled directly onto ships hulls. With every port in the world reportedly connected by only two ship voyages (Kaluza et al., 2010; Keller et al., 2011), and an estimated 3500 million tons of ballast water transferred annually through merchant shipping (Endresen et al., 2004), it is no surprise that shipping has been accredited for species introductions thousands of miles from their native ranges. Transport of species through ballast water has long been considered an important vector for NNS, having been hypothesised as early as 1903 (Ostenfeld, 1908, from Carlton, 1985), and has taken much of the research focus over the past 30 or so years (Bailey, 2015).

Ballast water is essential to a ship's function, serving to alter draft, trim and stability to maintain the safe operation of vessels at sea. Organisms are taken on board vessels during the planktonic larval phase of life cycles, within water taken into ballast tanks. Once in ballast tanks, water is generally preserved without mixing with any external water during transit, and only exchanged upon approach to the destination port (Carlton, 1985; Bailey, 2015). 
Maintaining this relatively constant environment within ballast tanks promotes survival of NNS larvae, compared to biofouling organisms settled directly onto ship hulls which must survive changes in environmental conditions during a ships voyage (Schimanski et al., 2016). Our understanding of the transport potential of ballast water has developed significantly following the initial work of Carlton (1985), with the focus of publications shifting from identifying ballast water as a vector for NNS to risk mitigation and management (Bailey, 2015).

Owing to the international nature of shipping, management plans must be a unified effort from a significant percentage of the world's sea trading nations to have any hope of reducing the invasion potential. Efforts to manage ballast water as a vector for NNS introduction first reached the forefront of international discussion at the United Nations Conference on Environment and Development (UNCED) in 1992, wherein it was written in Agenda 21 that an action of the International Maritime Organisation (IMO) would be to implement rules on ballast water discharge with the goal of preventing the spread of NNS (UNCED, 1992; Firestone and Corbett, 2005). 2004 saw the next big step towards global unity on ballast water management with the Ballast Water Convention (BWC), actioned by the IMO. This convention was pivotal in outlining clearly defined measures that can easily be implemented and enforced by member states to reduce the risk of NNS introduction through ballast water. Regulations that directly affect shipping activity are regulations 'D1' and 'D2' (IMO, 2004). Regulation 'D1' states that no less than 95\% of ballast water must be exchanged at least 200 nautical miles from shore, in water at least $200 \mathrm{~m}$ deep. Regulation 'D2' is a more stringent measure and states that ballast water may only be discharged if it meets a specific set of criteria focussed on the number of organisms and microbes (e.g. Escherichia coli and Vibrio cholera) present in ballast water samples. Proceedings of this convention began to be enforced on $8^{\text {th }}$ September 2017, stating all ships must conform to the 'D1' standard, and all new ships to the 'D2' standard. By 2024, all ships new and old must conform to the 'D2' standard. At the time of writing, $85 \mathrm{UN}$ member states have ratified this legislature, representing more than $90 \%$ of the world merchant shipping tonnage (IMO, 2020). There are notable absentees from that list, including the UK and the USA, however, ships from states absent from the list must still follow ballast water management procedures when entering any other ratifying state (IMO, 2020). 
Whilst the UK has not yet ratified the BWC, a recent update from the government has outlined that they are "committed to acceding to the Convention and implementing it into UK law', and that the scheduled 2020 launch of the consultation has been delayed 'to allow industry to focus on maintaining the flow of essential goods during the COVID-19 crisis' (Maritime and Coastguard Agency, 2020). Nonratifying states may also use the BWC as guidance in forming independent legislature, and indeed may build on the BWC to enforce more stringent management measures.

Whilst much of the recent published scientific literature on ballast water has focussed on methods to successfully and cost effectively treat ballast water, removing any organisms and pathogens (e.g. Tsolaki and Diamadopoulos, 2010; Wright et al., 2010; Pereira and Brinati, 2012; Lacasa et al., 2013), international efforts are still based heavily on the BWC. It is possible that over time the BWC will be amended to incorporate an appropriate treatment method, if one is successfully identified.

Biofouling is understood to be a less common mode of NNS introduction than through ballast water. Due to extreme changes in environmental conditions often experienced during voyages (Schimanski et al., 2016), this mode of introduction is more commonly associated with relatively short ship transits (e.g. between Northern European ports) and movements of recreational vessels which generally travel short distances (Murray et al., 2011; Martínez-Laiz et al., 2019; Ulman et al., 2019). Common fouling species tend to be resilient species, capable of surviving a wide range of physical and physiological stressors, traits that also make these species likely to become successful NNS after relocation (Olyarnik et al., 2009). Common among fouling organisms are barnacles which have proven successful in settlement on both organic and non-organic matter, including boat hulls, plastics, and even ID leg rings of migratory sea birds (Gollasch, 2002; Tøttrup et al., 2010; Li et al., 2016). The latter is a prime example of the potential invasiveness of certain species and particularly the ability to spread over small distances once becoming established within a region. This secondary spread of NNS is arguably the biggest concern regarding the biofouling vector as it is accelerating the invasion process and taking a species from 'introduced' to 'invasive' (Davidson et al., 2010; Roche et al., 2014; Martínez-Laiz et al., 2019). 
As discussed previously (section I.II.II.), biofouling on ship hulls can have significant economic ramifications regarding the efficiency of maritime trade (Schultz et al., 2011) and, along with guidelines surrounding the management of biofouling on commercial vessels (IMO, 2011), this has driven a conscious effort to reduce the amount of hull fouling. However, hull fouling on recreational vessels has remained a largely unregulated vector for the spread of NNS (Roche et al., 2014). Whilst there have been guidelines published specifically discussing the management of hull fouling on recreational vessels (IMO, 2013) there can be a significant lag period between the time when guidelines are published and the guidelines actually being enforced widely by member states, as we have seen with regard to the Ballast Water Convention. As our understanding of hull fouling as a vector for the spread of NNS has increased there has been a shift in the focus of research efforts towards identifying factors which may contribute to hull fouling as well as techniques to effectively manage this vector (Dafforn et al., 2009; Coutts et al., 2010; McDonald et al., 2014; Roche et al., 2014; Schimanski et al., 2016, 2017).

\section{I.III.II. Corridors (canals)}

Introductions via canals are deemed to have occurred independently to shipping activities along these waterways, with organisms gradually expanding their range through a canal and into an adjoining body of water rather than having been directly transported by vessel movements. The construction of canals however is directly related to maritime trade. A review into the pathways of marine species concluded corridors to be the third most common pathway for species introduction worldwide, accounting for $17 \%$ of all introductions (Molnar et al., 2008). However, a similar study with a European focus has since revealed corridors to be the second most common pathway (Katsenevakis et al., 2013). The difference between these two studies has been directly associated with the role that the Suez Canal plays in introducing NNS to the Mediterranean Sea (Katsenevakis et al., 2013). Almost all species introductions via canals in Europe can be attributed to the Suez Canal which, following various structural modifications since its initial construction, has provided a corridor whereby water conditions are not unfavourable to the gradual passage of organisms either as adults or through larval dispersal (Rilov and Galil, 2009; Katsanevakis et al., 2013). 
Indeed, it is thought that over half of the NNS in the Mediterranean Sea were introduced via the Suez Canal (Galil et al., 2014). Concern over the likelihood of future invasions through the Suez Canal has been strongly voiced in recent years (Galil et al., 2015ab) and continues to be a key feature of research into biological invasions within the Mediterranean (Galil et al., 2017, 2021; Tsiamis et al., 2020).

\section{I.III.III. Aquaculture}

There are two ways in which aquaculture can serve to transport species around the world. Firstly, there is the direct transport of target species for cultivation in aquaculture farms, and secondly there is the indirect transport of associated species which may be present along with target species. The Pacific oyster, Magallana gigas, is one example of a target species which has become established and invasive outside of the culture areas. M. gigas was introduced for aquaculture numerous times through the latter half of the $20^{\text {th }}$ Century and has since established populations across Europe (Streftaris and Zenetos, 2006; Lallias et al., 2015). The indirect spread of species associated with target species tends to occur when the shipment is not thoroughly cleaned and is frequently associated with the spread of macroalgal species (Geburzi and McCarthy, 2018). The introduction and spread of Sargassum muticum around Europe, for example, has been related to the transport of M. gigas for culture (Critchley et al., 1983; Harries et al., 2007). Ecosystem engineering organisms, such as reef builders, that are transported for aquaculture purposes (such as M. gigas) are of particular concern regarding the unintentional transport of additional species as these species are able to provide a favourable habitat for the colonisation of additional species and therefore increase the chance of successful introductions (Ruiz et al., 2000; Markert et al., 2010).

Of all the pathways for species introductions that have been and will be discussed here, aquaculture is probably the most well controlled and managed, which is due in part to the strict licensing surrounding the establishment of an aquaculture facility (Savini et al., 2010). There are also various well-established pieces of European legislation surrounding the use of non-native species in aquaculture, which outline risk assessment and risk mitigation procedures to assist in the control of this vector (e.g. EU, 2007; EU, 2008). 
These control measures are thought to have contributed to the observed decline over the years in the number of non-native species being introduced through aquaculture in Europe (Katsenevakis et al., 2013).

\section{I.III.IV. Additional vectors}

Aside from the three primary vectors described above, there are a range of other vectors that are considered to be less of a threat for species introductions globally. Of these smaller vectors the aquarium trade is generally considered to be the biggest introducer of species worldwide and considered to be the fourth most significant pathway behind shipping, corridors and aquaculture (Molnar et al., 2008; Katsanevakis et al., 2013). Literature addressing the aquarium trade specifically as a vector for NNS that should be monitored, regulated and managed only really emerged in the early to mid-2000s (e.g. Padilla and Williams, 2004; Gertzen et al., 2008) despite research highlighting aquariums as sources of NNS introduction being published in the 1990s (Meinesz and Hesse, 1991; Mills et al., 1993). Much of the published literature on this subject focusses on freshwater species, owing largely to the greater number of introductions within the freshwater environment and the greater number of freshwater fish available on the global aquarium market (MacedaVeiga et al., 2013; Tricarico et al., 2017). Most notable of all aquarium based NNS introductions within the marine environment is that of Caulerpa taxifolia within the Mediterranean Sea. C. taxifolia is a species of Chlorophyta used commonly in the aquarium trade as a decorative plant, and successfully invaded the Mediterranean Sea after initially being introduced to the region from an aquarium in Monaco in the mid1980s (Meinesz and Hesse, 1991; Boudouresque and Verlaque, 2002). As a case study for the progression of an organism from introduced to invasive, $C$. taxifolia within the Mediterranean offers one of the most comprehensively studied and documented accounts of spread through a region, having been monitored for over 30 years since the initial introduction and has now been recorded as prolific in the western Mediterranean (EEA, 2012). Indeed, without such a highly studied and highprofile example of an aquarium-based introduction, this mechanism of introduction may not have been considered an important factor to regulate within invasion biology as early as the 1990 s - 2000s. 
An additional vector which has been gathering increased research attention over the last decade or so is rafting. Rafting is a means of transport almost exclusively for fouling organisms, which colonise materials and drift across water bodies to become introduced in a non-native range (Gregory et al., 2009). This vector has been associated with dislodged man-made structures (e.g. buoys and pontoons) which may then drift into new locations (Astudillo et al., 2009; Carlton et al., 2017). Carlton et al. (2017) report the transport of 289 Japanese coastal species to North America and Hawaii over 6 years following damage caused by the 2011 East Japan earthquake and subsequent tsunami. Recently, there has been a large focus on marine litter as a rafting vector (Gregory et al., 2009; Rech et al., 2016, 2018; Miralles et al., 2018). Historically rafting as a vector for NNS dispersal would have been possible on natural debris such as fallen trees, however, the extreme increase in the amount of marine litter in recent decades has thrust this vector to the forefront of invasion research (Gregory et al., 2009) and is particularly concerning given the unpredictability and difficulty in monitoring, controlling and managing introductions. Indeed, it is thought that rafting is the third most significant pathway for species introductions into British marine and brackish waters, behind shipping and aquaculture (Minchin et al., 2013).

\section{I.IV. Management of NNS}

Given the potentially significant impacts that NNS can have on the environment and economy it is understandable that the management of NNS has been a key focus in the literature over the last two decades. Initial efforts to outline the most effective management strategies focussed on the stage of invasion, with different management schemes available at different stages of invasion (Blackburn et al., 2011). A species in the establishment stage of invasion may, for example, be managed through containment to limit the spread whilst a species in the spreading stage may only be managed through impact mitigation or, rarely, through eradication (Blackburn et al., 2011). However, this is a 'unified framework' and is designed with both terrestrial and aquatic NNS in mind. 
Management of aquatic NNS can be considerably more difficult than that of terrestrial species. This is in part due to the difficulties in monitoring for aquatic introductions compared to terrestrial, but also due to the reproductive traits of many aquatic species (Geburzi and McCarthy, 2018). A number of reproductive traits have been identified as common among successful aquatic NNS. Planktonic larval stages can increase dispersal and play an important role in the secondary spread of a species following an initial introduction, enabling the species to move rapidly through the invasion process, with some NNS displaying prolonged larval stages by comparison to native species which further increases the dispersal potential (Viard et al., 2006; Delaney et al., 2012; Geburzi and McCarthy, 2018). Furthermore, aquatic NNS in general favour an r-selected reproductive strategy (with early maturation, short generation time, high fecundity, and rapid growth rates). A number of species have also been found to express a degree of plasticity in reproductive strategy by alternating between $\mathrm{r}$ - and $\mathrm{k}$-selected strategies, enabling these species to take advantage of a range of different ecological scenarios to maximise the chance of establishment (Sakai, 2001). As such, eradication is rarely a successful management technique in aquatic systems (Sambrook et al., 2014; Cook et al., 2015; Booy et al., 2017). The opinion on what may be considered 'successful' management of an invasion differs between terrestrial and aquatic systems. In both systems the ultimate goal of management is to eradicate the non-native species, however for the reasons explained above this is rarely achieved in aquatic and especially marine. Successful management of invasive species in aquatic systems will often take the form of containment, halting the spread of a species.

Much of the focus for management of aquatic NNS has taken a species specific, case study style approach (e.g. Anderson, 2005; Folino-Rorem and Indelicato, 2005; Coutts and Forrest, 2007; Sambrook et al., 2014;). However, a recent review of management strategies in marine systems has suggested an alternative generalised approach to the decision-making process, whereby appropriate management actions for any given species can be chosen based on a functionally similar model species (Giakoumi et al., 2019). This method would reduce the time spent in deciding upon an appropriate management action and in turn maximise the chance of successfully managing the NNS (Giakoumi et al., 2019). 
Whilst the approach to NNS management has been subtly changing over the years, one theme has remained a constant feature in every approach; management strategies are always most effective following an early detection of a species introduction (Blackburn et al., 2011; Dahlstrom et al., 2011; Giakoumi et al., 2019; Boon et al., 2020). This is particularly important if an aquatic species is to be targeted for eradication (Sambrook et al., 2014; Booy et al., 2017). It follows then that a key aspect of management is the regular and routine monitoring for NNS introductions.

\section{I.V. Methods of monitoring NNS}

There are countless methods for monitoring NNS, either to detect new species introductions or to monitor established NNS. Methods typically vary based on the environment which is being monitored and the types of species which are being targeted. The focus of this thesis is on the identification of NNS within port fouling communities and this section will therefore share a similar focus.

Common methods for surveying fouling communities and identifying NNS that have been used extensively since the late $20^{\text {th }}$ century are settlement surveys and rapid assessment surveys. Settlement, or colonisation, surveys generally take the form of a settlement tile or settlement panel which is suspended in the water for a period of weeks or months before being retrieved (e.g. Sutherland and Karlson, 1977; Dobretsov et al., 2005; Bangor University, 2015; Leclerc et al., 2019). Upon retrieval, organisms that have colonised the material are identified to provide an understanding of the fouling community within the surveyed environment. Settlement panels can vary in material, size, shape, and colour depending on the specific research questions being answered. For example, there have been a number of studies published which investigate the effect of orientation on the formation of fouling communities, in which case different shaped settlement panels may be deployed (e.g. Glasby, 2000; Glasby and Connell, 2001; Floerl et al., 2012; Bangor University, 2015;). Most studies however use a plastic settlement panel in the size range of $170-400 \mathrm{~cm}^{2}$ (e.g. Sutherland and Karlson, 1977; Glasby, 2000; Glasby and Connell, 2001; Bangor University, 2015; Collin et al., 2015). 
Rapid assessment surveys (RAS) differ from settlement surveys in that they take advantage of materials which are already present within an environment and observe the fouling community which has been forming over a number of years or even decades (Cohen et al., 2005; Arenas et al., 2006; Collin et al., 2015; Rohde et al., 2017). RAS serve to quickly survey sites of interest, focussing on structures of particular interest to invasion biologists such as artificial structures including pontoons, mooring ropes, and buoys (e.g. Cohen et al., 2005; Arenas et al., 2006). This type of survey can be either shore based, by taking advantage of low tides or by removing materials from the water for access, or it can apply the use of divers and underwater video techniques (Marraffini et al., 2017).

Direct comparisons between each survey type has proven both methods to be successful in describing fouling communities and identifying NNS (Marraffini et al., 2017; Bangor University, 2015), however each does have advantages over the other which can be important factors in determining which method is most appropriate for a given research goal. Settlement surveys offer a standardised methodology, allowing for year on year comparisons when monitoring communities, as well as taking into account smaller and more cryptic species a lot more easily than in RAS, ultimately leading to the identification of more species (Marraffini et al., 2017; Rohde et al., 2017). Settlement surveys are also frequently less labour intensive than RAS, particularly when the RAS utilises diver surveys (Marraffini et al., 2017). RAS are however quicker, hence 'rapid' assessment, require less planning, provide insight into colonisation across a range of different material types, and are generally cheaper than settlement surveys (Rohde et al., 2017). A combination of the two survey types is considered to be the most robust approach to survey fouling communities (HELCOM, 2013; Rohde et al., 2017). Both of these methods however rely heavily on the ability to accurately taxonomically identify organisms, either in situ or in a laboratory, which often necessitates a significant time commitment of expertise to enable continued monitoring.

More recent approaches to invasion biology adopt molecular techniques. With the development of Next Generation Sequencing (NGS) and the discovery of environmental DNA (eDNA) as a source of both invertebrate and vertebrate DNA, there has been an increased application in invasion biology (Ficetola et al., 2008; Rollins, 2015; Goldberg et al., 2016). 
NGS applications in this field are broad, enabling researchers to identify the origins of non-natives, identify species present in an environment using eDNA metabarcoding, and to investigate in more detail the genomic traits associated with successful NNS (Rius et al., 2015). For many researchers, NGS offers a solution to the need for time investments and taxonomic experience in lab analyses and can also save time in field sample collection as samples can be handled with less care as would be needed to accurately identify species visually. Currently there are two primary reasons stopping NGS from taking over as the chief method underpinning non-native research: the cost, and the need for a more complete record of species genomes. I have no doubt that as these two concerns are addressed then molecular approaches, primarily utilising eDNA metabarcoding, will become the primary method for monitoring of NNS. There has indeed already been research published to suggest the overall cost of metabarcoding is less than that of a traditional visual survey methods (Borrell et al., 2017).

\section{I.VI. Port and marina research}

Ports and marinas are of particular interest within the field of invasion ecology, owing primarily to the integral role these sites play in international shipping and recreational vessel movements, which we know to be key vectors for NNS dispersal (section I.III.I.; Carlton and Geller, 1993; Katsanevakis et al., 2013; Seebens et al., 2013; Bailey, 2015; Martínez-Laiz et al., 2019). Ports serve as the most likely and often most common location for NNS to settle after being transported directly into these locations through shipping (Seebens et al., 2013). Provided the environmental conditions in a given port or marina are suitable for an introduced species to survive, the abundance of artificial structures and often sheltered environment offer ideal habitats for NNS to thrive (Mineur et al., 2012; Foster et al., 2016). Marinas in particular are also relatively easy places to access to carry out surveys, by comparison to intertidal and subtidal rocky shorelines, and have therefore been the focus of numerous studies into NNS (e.g. Arenas et al., 2006; Borrell et al., 2017; Marins et al., 2010; Marraffini et al., 2017). For these reasons, several longterm NNS monitoring programmes have been established in ports and marinas around the world, following the early work of Hewitt and Martin (1996, 2001). 


\section{I.VI.I. Quantified literature search into port- and marina-based surveys}

Despite the level of legislature surrounding commercial shipping by comparison to recreational vessels (section I.III.I.) there is a marked disparity in the amount of published research conducted in ports and marinas, whereby the majority of peer-reviewed publications with a focus on NNS are based on research conducted within marinas rather than ports. Whilst there is no known previous mention in the literature as to why marinas are studied more than ports, I suggest the reason is more to do with a lack of accessibility rather than a lack of research interest.

To quantify this, I have conducted a quantified literature search pooling published research based on a series of search terms (Figure 2) using the Web of Science. The initial pools of search results were filtered down based on relevance to this research topic and, importantly, whether the publication featured primary data collection from within either marinas or ports. An initial search focussing on marinas and harbours yielded 425 publications, of which 118 were deemed relevant, compared to an initial 247 for ports of which only 38 were relevant. Rather strikingly, of the 38 studies conducting research either wholly or partly within port environments 15 were based in Europe, of which none contained data collected from UK ports. Further to this, of the 15 European based studies, 6 were associated with a recent initiative termed the 'Port Baseline Survey' (PBS) initiative which acts to improve the understanding of a range of aspects of port communities in the Adriatic (Di Poi et al., 2019; Kraus et al., 2019; Mandic et al., 2019; Petrocelli et al., 2019; Travizi et al., 2019; Vidjak et al., 2019). This is a very positive initiative; however, it does mean that 6 of the 15 European based studies feature data collected from the same (up to 12) Adriatic ports. Clearly there is the need for further initiatives similar to the PBS.

It is worth noting that there are several technical or industry reports featuring data collected within ports or marinas worldwide (e.g. Eno et al., 1997; Floerl et al., 2012; Bangor University, 2015; Hurst, 2016). These are generally commissioned and carried out by government bodies and therefore often fall outside of the realm of peer-reviewed publications, which means they do not feature within the literature search. Again, these are weighted toward the collection of data from marinas rather than ports, which is particularly the case within the UK. 
a)

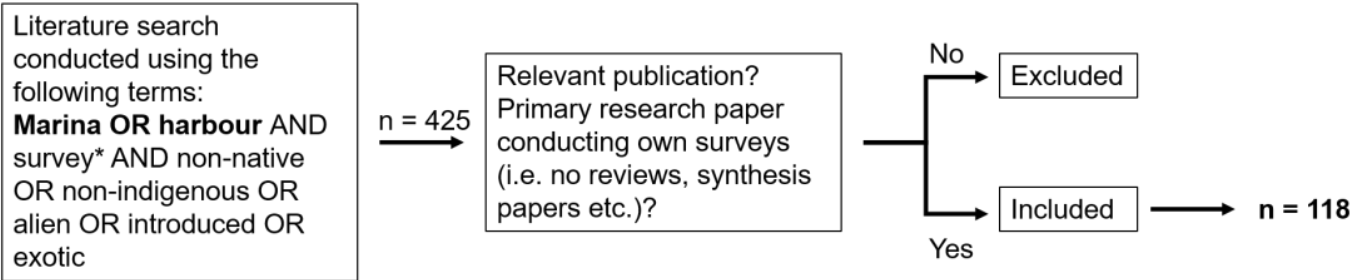

b)

Literature search conducted using the following terms:

Port AND survey* AND

non-native OR non-

indigenous $\mathrm{OR}$ alien $\mathrm{OR}$

introduced OR exotic

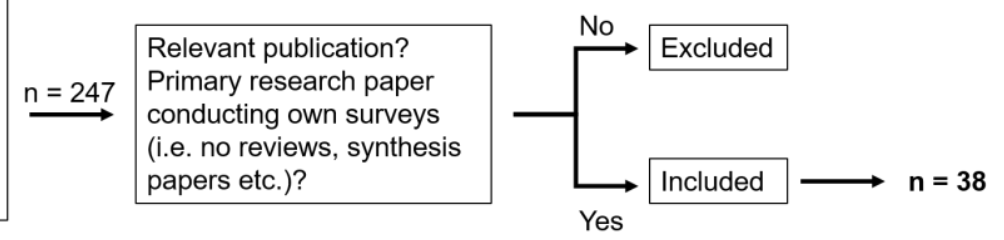

Figure 2. Schematic representation of the search criteria and selection process involved in finding literature relevant to the literature search described in section I.VI.I.

\section{I.VI.II. The ports of South Wales, UK}

There are six active ports along the South Wales coast. From east to west these are the ports of Newport, Cardiff, Barry, Port Talbot, Swansea, and Milford Haven (Figure 3). Milford Haven is the only port of the six to be controlled by an independent organisation, the Milford Haven Port Authority, whereas the remaining five are all owned and run by Associated British Ports (ABP). ABP is the largest UK port authority and has control over 24 ports in England, Scotland and Wales. This body of research has been part funded by and completed in collaboration with ABP, and as such will focus on the five ABP operated South Wales ports of Newport, Cardiff, Barry, Port Talbot and Swansea. 


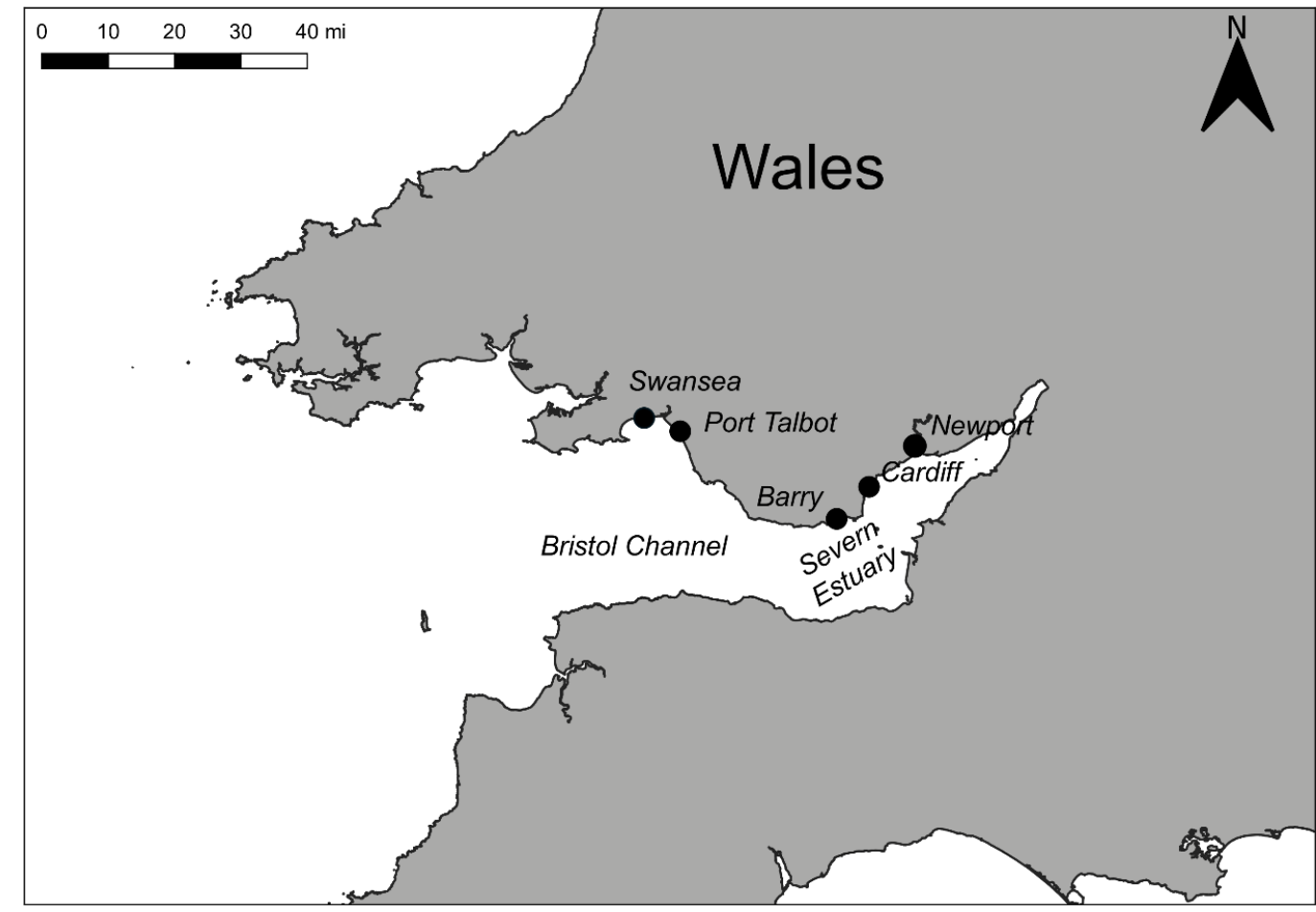

Figure 3. Location of five Associated British Ports within South Wales.

These ports are all active today, although to a varying degree. In terms of cargo tonnage handled per year by each port, Newport handles over 1.8 million, Cardiff over 1.8 million, Barry over 300,000, Port Talbot over 6.6 million, and Swansea over 520,000 (Associated British Ports, 2020). These ports all comprise of a series of enclosed docks which are isolated from the adjoining water body (Bristol Channel or Severn Estuary) by a lock (Figure 4). This means that the habitats within the ports are all subtidal up to a depth of at least $10 \mathrm{~m}$ in all active shipping areas.

Despite the fact that ports are known to be high risk areas for the introduction of NNS and that there is active legislation outlining efforts to detect, manage and mitigate the impacts of NNS (Dahlstrom et al., 2011; UK Government, 2015; Boon et al., 2020), there has been very little information published as to the organisms known to inhabit these ports. Across all five ports, there have been two research papers published, back in the mid-1900s, describing aspects of the subtidal community in the Port of Swansea (Naylor, 1957, 1965). Naylor (1957) recorded the first UK occurrence of the crab Brachynotus sexdentatus as well as making some reference to other species identified during surveys, before publishing the results of a more thorough survey of benthic fauna nearly 10 years later (Naylor, 1965). 
There are additional mentions of the Port of Swansea in further publications relating to NNS within the UK, however these are short remarks of no more than a sentence or two and relate to the non-native bryozoan Bugula neritina (Ryland, 1960; Eno et al., 1997; Arenas et al., 2006). Outside of the peer-reviewed literature there have been four recorded occurrences of the non-native amphipod Gammarus tigrinus (three in Port Talbot, one in Newport; NBN Atlas, 2020). These sporadic pieces of information comprise the entire baseline knowledge of port fouling communities within the ports of Newport, Cardiff, Barry, Port Talbot, and Swansea. Outside of the information provided by Naylor from the Port of Swansea there is also no information on the native species that inhabit each port, which would be essential in understanding the role NNS play in these communities and the extent with which NNS may impact these environments.

Collaborating with ABP has offered a unique opportunity to work extensively within these ports to fill in some glaring gaps in the monitoring of non-native species, develop effective survey techniques for use within active ports, and generally advance our understanding of the role ports may play in the introduction of NNS within the UK. 


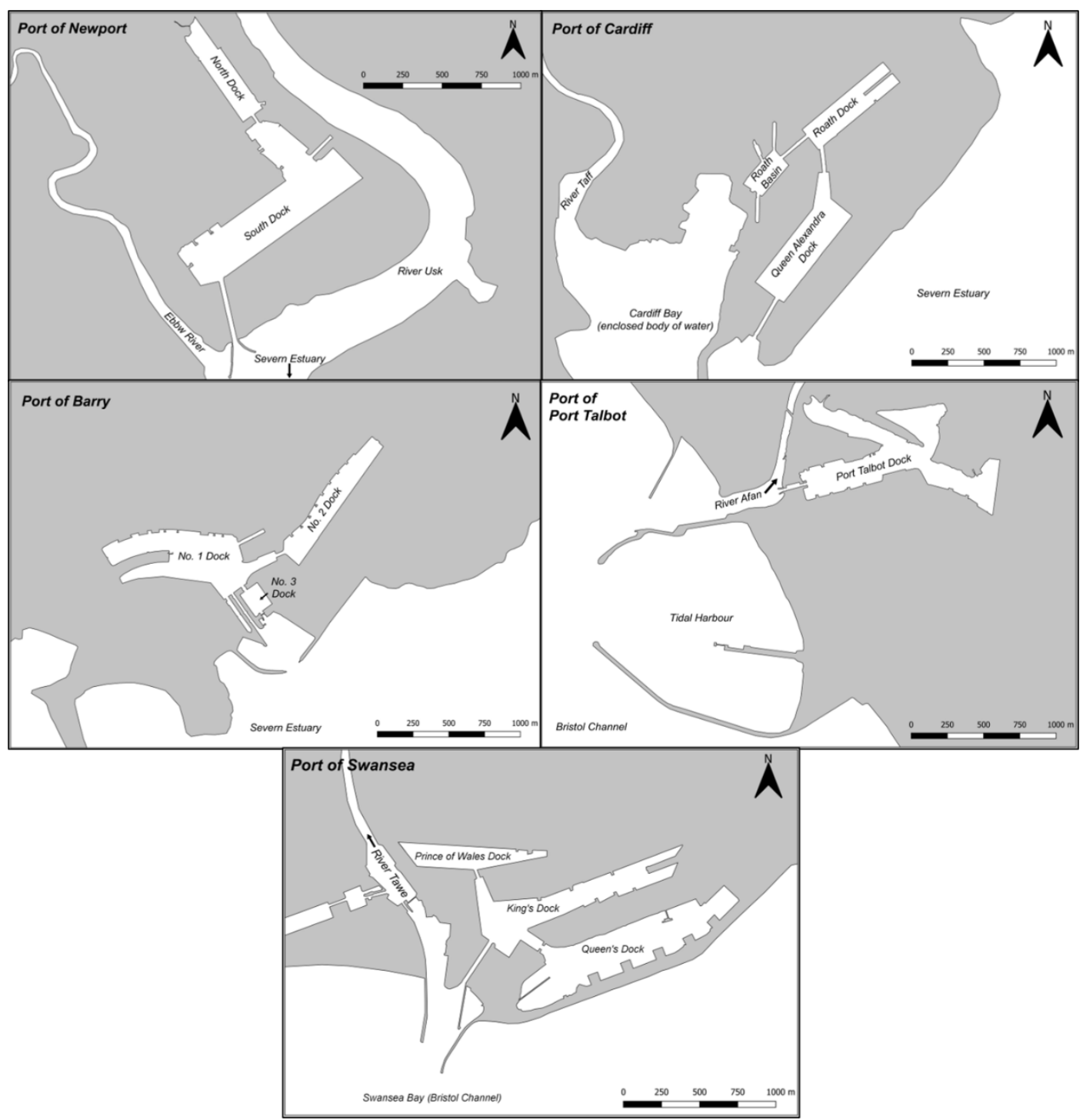

Figure 4. Map of each of the ports of Newport, Cardiff, Barry, Port Talbot and Swansea showing the position of individual docks within each port and the position relative to either the Bristol Channel or Severn Estuary. 


\section{THESIS AIMS AND OBJECTIVES}


As we have seen, the monitoring of non-native species (NNS) is essential in developing and implementing the most effective schemes of management (Blackburn et al., 2011; Giakoumi et al., 2019). Whilst ports are regarded to be one of the highest risk areas for aquatic species introductions these areas are rarely studied, to the point that we often do not have any records of either native or non-native species from within many ports (section I.V.I.). There have been a small number of successfully implemented long-term monitoring programmes within ports around the world, including in Australia, New Zealand and Orkney, Scotland (Hewitt and Martin, 1996, 2001; Inglis et al., 2005; Campbell et al., 2007; Kakkonen et al., 2019) which provide valuable insight into the introduction and spread of NNS in and around ports. The implementation of such programmes is however rare. There is legislation outlining a duty for the managers of ports, marinas and associated infrastructure to document the NNS that are present within their waters (Dahlstrom et al., 2011; Boon et al., 2020), however there is no guidance provided on how this data can be collected and no standardised survey methodology that can be implemented. It is no real surprise then that the resolution and quality of NNS data is poor from within port environments in the UK.

Ports are not easy places to work in. These are heavily industrialised, busy, and generally quite hostile places to conduct surveys by comparison to most other coastal locations and coastal infrastructure. Many of the commonly used survey techniques used to describe fouling communities and identify NNS (see section I.V.) cannot be used effectively within port environments. Diver surveys can be ruled out based on safety regulations, rapid assessment surveys are inhibited by the lack of access to submerged materials, and even settlement studies can be limited in their use due to a lack of suitable locations for deployment amongst active working vessels (personal observation). The only comprehensive guide outlining in detail the survey procedures necessary to identify NNS within port environments is that of the joint HELCOM/ OSPAR guidelines which, despite being focussed largely on the monitoring of ships' ballast water, does recommend the combined use of settlement surveys and rapid assessment to identify NNS within port fouling communities (HELCOM, 2013). These guidelines do however refer to the fact that rapid assessment surveys may not always be feasible for use within active ports (HELCOM, 2013). 
I would further suggest that the use of settlement surveys in the conventional form, using single settlement tiles, may not be the most effective use of the limited space available within ports. The joint HELCOM/ OSPAR guidelines are also not adopted by the UK to offer a standardised methodology for port surveys, which remains an issue to be resolved.

As such my key objectives were as follows:

1. Design a survey methodology which can be used to identify NNS within active ports, and which may serve in the future as a standardised method across numerous ports

2. Provide a baseline record of the species which form fouling communities within the ports of Newport, Cardiff, Barry, Port Talbot, and Swansea

3. Identify and report the presence of any non-native species present within these ports

4. Develop an understanding of the formation of fouling communities within port environments, encompassing elements such as rate of colonisation, community succession, and differences in colonisation between substrate types, all of which may assist in the future management of NNS

5. Evaluate the use of plastic as a substrate within settlement surveys

6. Produce a NNS field guide tailored for use by port employees which contains all NNS recorded from each port

Chapter 1 seeks to develop a tailored survey methodology targeted at describing fouling communities and identifying NNS, specifically from within active port environments. I designed two settlement surveys which will offer insights into different aspects of colonisation and the formation of fouling communities, and importantly overcome some of the difficulties that ports present for conventional survey methods. This chapter can be seen as a preliminary study, trialling the use and effectiveness of these new surveys within the Port of Swansea. Objective 1 is primarily addressed within this chapter, with an element of objectives 2 and 3 but for the Port of Swansea only. This chapter has been accepted for publication in the Journal of the Marine Biological Association of the UK and is currently in print. 
Chapters 2 and 3 focus on objectives 2, 3 and 4, whereby the survey methods trialled in Chapter 1 are deployed across all five ports. Chapter 2 focusses specifically on one survey method, the Successional Settlement Survey, and chapter 3 on the second survey method, the Mixed Material Survey. Chapter 2 has been submitted for publication to Estuarine and Coastal Shelf Science.

Chapter 4 can be considered as a control study aimed at validating the use of plastic as a substrate in settlement surveys, as has been used extensively in previous studies (section I.V.). Our increased understanding of the effects of plastic on the development of marine organisms (Avio et al., 2017; Haegerbaeumer et al., 2019; Gardon et al., 2020) has raised doubts over the use of plastics to accurately represent natural fouling communities. For this chapter I deployed an adapted survey to quantify any differences in the colonisation of plastic and natural (slate) substrates. This chapter specifically addresses objective 5 .

Objective 6 is addressed in Appendix IV and takes the form of a field guide. This is not therefore directly a data chapter but instead provides a synopsis of all the NNS identified across the four data chapters. This field guide has been given to ABP and will be used not only within their South Wales ports but potentially in all 24 of their UK ports to promote the passive monitoring and recording of port NNS. 


\section{CHAPTER 1: Design and implementation}

of two surveys targeted at describing fouling communities and identifying nonnative species within active ports*

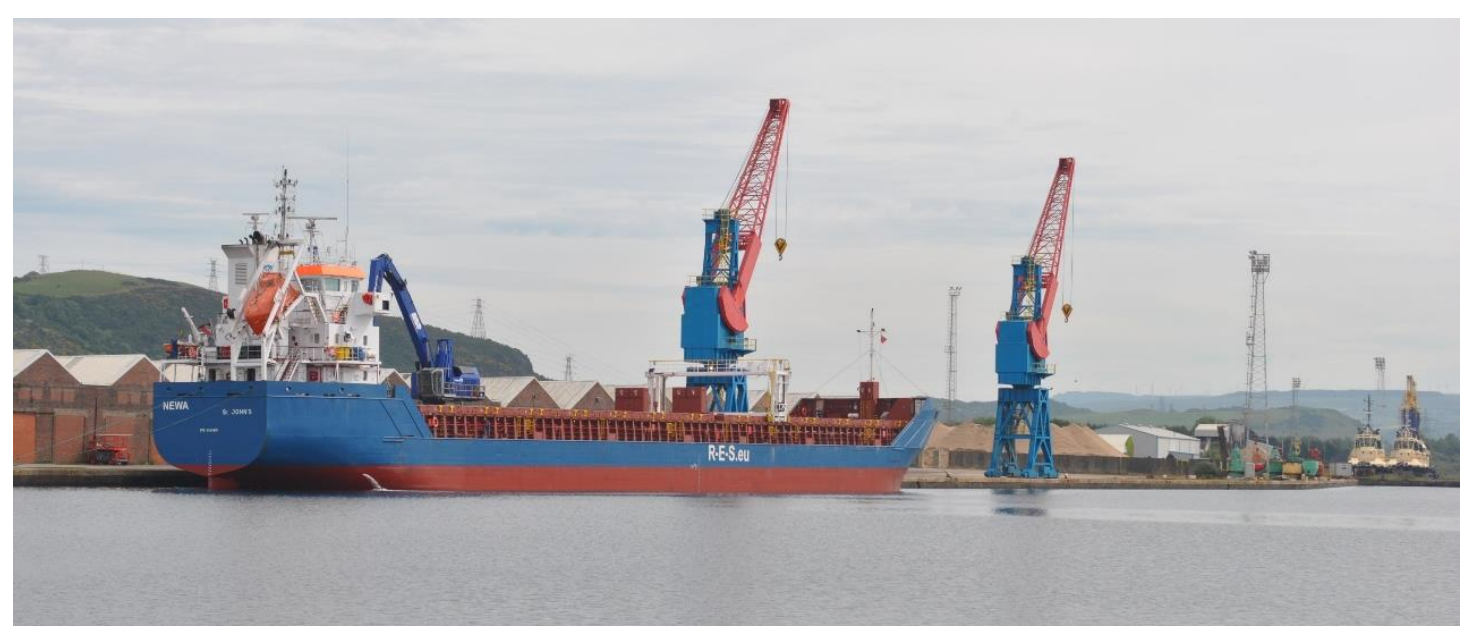

*A version of this chapter has been published as: Holmes, $\mathbf{S}$ and Callaway, R., 2020.

Design and implementation of two surveys targeted at describing fouling communities and identifying non-native species within active ports. Journal of the Marine Biological Association of the UK. 100, 1191-1204. 


\subsection{Abstract}

Ports have long been considered 'high-risk' areas for the introduction of nonnative species (NNS) and should therefore be a focus of NNS monitoring. The industrial nature of active ports can, however, provide various problems when attempting to carry out monitoring programmes. Current methodologies designed to identify NNS and to describe fouling communities have not been developed specifically for use in active ports and can encounter a number of issues when used in these environments. Here, two surveys were developed and trialled within an active port in South Wales, UK, designed to describe fouling communities, identify NNS and overcome some of the major limitations to conducting surveys within ports. Over a six-month period, fouling communities dominated by solitary ascidians developed in each survey. Seven NNS were identified, mostly species already recorded in the 1950s, including the Mediterranean crab Brachynotus sexdentatus, and the more recently introduced Japanese skeleton shrimp Caprella mutica. Each survey was evaluated independently with respect to key factors, including the ability to detect NNS and practical aspects of using these survey methods in an applied context. I conclude that whilst each survey can function independently, the use of both survey types in conjunction offers the most robust solution to identifying NNS and describing wider fouling communities within active ports. This research has implications for the future monitoring and management of NNS within UK ports.

\subsection{Introduction}

Non-native species (NNS) have long been considered as one of the biggest threats to biodiversity, the stability of marine communities, and ecosystem functioning (Sala et al., 2000; Bax et al., 2003; Molnar et al., 2008; McGeoch et al., 2010; Rohde et al., 2017). NNS can give rise to significant ecological and economic damages, however, a major concern is their high variation and unpredictability of impacts (Pimentel et al., 2001; Lovell and Stone, 2005; Katsanevakis et al., 2014). Coupled with this is the differing nature of impacts, for example both positive and negative impact at the ecosystem service level rather than the overall perceived impact of species (Katsanevakis et al., 2014). 
In general, the preferred approach is to prevent the introduction and spread of NNS rather than to undertake expensive eradication or control measures post establishment (Puth and Post, 2005; Pyšek and Richardson, 2010; Rohde et al., 2017). Implementing effective monitoring programmes to identify the arrival of NNS, serving as an early warning, is key in preventing establishment (Anderson, 2007; Rohde et al., 2017).

Marine organisms have likely been transported and become established around the world for thousands of years (Aubet, 2001; Carlton and Hodder, 1995). However, globalisation has led to the rapid increase in species introductions observed over the last few decades (Streftaris et al., 2005; Floerl et al., 2009; Hulme, 2009; Maceda-Veiga et al., 2013; Sardain et al., 2019). Maritime trade has long been recognised as the primary invasion vector for marine NNS (Ruiz et al., 1997; Katsanevakis et al., 2013; Williams et al., 2013; Bailey, 2015), either through ballast water or hull fouling, meaning that ports are considered to be more at risk of invasion by NNS than natural coastal habitats.

Despite the strong link between ports, maritime trade and NNS, there is relatively little published research aimed specifically at describing communities within ports (Bailey, 2015). This may be due in part to the limitations in terms of ease of access and safety when working within active ports, as well as the lack of need or desire for port owners to publish any findings from private surveys that may have been undertaken within their ports. By contrast, marinas are frequently studied worldwide as habitats for NNS (Canning-Clode et al., 2013; Guerra-García et al., 2015; Foster et al., 2016; Shenkar et al., 2018). Whilst marinas offer more accessibility and safety than ports, the habitats and factors influencing communities are often different even to the nearest port. Marinas are more commonly associated with the local spread of NNS through recreational boating (Martínez-Laiz et al., 2019), rather than being the initial site of species introduction, which should be the focus when attempting to prevent invasions. Effective management of NNS is made much more difficult when there is a lack of survey data (Campbell, 2011; Dahlstrom et al., 2011; Azmi et al., 2015), highlighting the importance of establishing long-term local and regional monitoring efforts. This should be focussed on the most likely sites for novel introductions which, in most cases, are ports that are linked to the global maritime trade network. 
Various methodologies for the monitoring of fouling communities and associated NNS have been trialled and published over the last few decades (e.g. Cohen et al., 2005; Arenas et al., 2006; Floerl et al., 2012). Rapid assessment surveys (RAS) are a favoured method and have been successfully applied in ports and marinas around the world (Cohen et al., 2005; Mineur et al., 2012; Bishop et al., 2015). However, the industrial nature of ports can provide difficulties when attempting to safely conduct this type of survey. Traditionally, RAS have targeted existing submerged structures (e.g. pontoons, buoys, ropes and chains; Cohen et al., 2005; Arenas et al., 2006; Mineur et al., 2012), where well-established fouling communities can be surveyed without the need to deploy some form of settlement material. This type of survey benefits from being a quicker and cheaper method than most alternatives, and it covers a range of different structures, materials and habitats. It is, however, difficult to record small and cryptic organisms which may be inhabiting structures as the rapid assessment does not use destructive sampling (Rohde et al., 2017). Further, it is not feasible to compare colonisation quantitatively among sites due to the non-standardised area units. In larger and more active ports, RAS may not always be a viable option due to the lack of long-term submerged structures, safe access to suitable sites, and port health and safety regulations.

Settlement and colonisation experiments are another chosen method for surveying fouling communities. This method has been heavily used over the past few decades and has been adapted into various designs, using a range of materials and deployed in a range of environments (Floerl et al., 2012; Bangor University, 2015; Cook et al., 2015). Generally, some form of plastic is used as a virgin settlement surface for larval settlement and development due to the low cost and weight of this material, with most survey designs applying a single plastic tile suspended in the water column and deployed for a period of several months. The advantage of this survey type is the ability to record quantified data and the option to choose suitable sites for deployment, which is particularly beneficial for use within active ports. The need for an extended deployment period with settlement surveys, often at least three or four months, and the associated higher costs that follow, are the main reasons why RAS have increased in use over the last couple of decades. 
Various studies have applied both RAS and settlement experiments, either in an effort to compare the accuracy of each method or to provide a more robust survey (Cook et al., 2015; Hurst, 2016; Marraffini et al., 2017). Perhaps the most important finding when comparing the two methods within the same study is the accuracy of identifying NNS (Lehtiniemi et al., 2015). Comparisons have shown that both settlement surveys and RAS are liable to miss certain NNS but are reliable at identifying the majority of NNS present (Cook et al., 2015; Marraffini et al., 2017). Cook et al. (2015) reported that settlement surveys and RAS each missed two species which were found in the other survey type, suggesting that the most robust surveys would incorporate elements of each survey type. Arguably, the most comprehensive guide for surveying within ports is the HELCOM/ OSPAR combined strategy targeted for use within the Baltic Sea (HELCOM, 2013). Whilst this strategy suggests a preference for the combined use of RAS and settlement surveys, it concedes that RAS may not be a viable option in all ports. Despite this, no alternative adapted survey type is offered, with it being suggested that a traditional form of settlement survey alone would be sufficient (HELCOM, 2013).

It follows that both survey types could be adapted for use within ports, to include beneficial traits of each whilst overcoming some of the limitations to working within active ports. These modified surveys could also provide some key information which currently neither RAS nor settlement experiments offer and yet which may prove valuable in informing targeted biosecurity plans (e.g. colonisation rate and community succession over the deployment period).

The aim of this research was to design a survey method tailored to describe the fouling community within an active port, focussing on identifying non-native species that may be present. The objectives were to:

a) quantify the succession of faunal colonisation,

b) compare colonisation success and fouling communities among different sites within the port,

c) identify differences in faunal colonisation between materials typically present in ports 
Two survey methods were developed and tested in an active port, the Port of Swansea, Wales, UK. The relative success of each survey method was assessed with respect to understanding the fouling community and detecting NNS. The potential role of the surveyed port as a vector for NNS into the region was considered.

\subsection{Materials and methods}

\subsubsection{Study area}

Research was conducted within the Port of Swansea, South Wales, UK. This port is an enclosed area consisting of three connected docks linked to the Bristol Channel via a lock. The oldest of these three docks, the Prince of Wales Dock, was constructed in the late $19^{\text {th }}$ century with the other two docks, King's Dock and Queen's Dock, being constructed in the early $20^{\text {th }}$ century. Historically the Port of Swansea has traded largely in copper, coal, tinplate and oil, of which only coal remains to be traded today following a decline in trade throughout the $20^{\text {th }}$ century. Along with coal, the port now regularly trades in dry bulks, scrap metals, timber, and general cargo, as well as having an aquaculture production site designated within Queen's Dock for the culture of blue mussels (Mytilus edulis). Around 600,000 tonnes of cargo are traded annually with an average of 81 ships per week transiting in an out of the lock, including pilotage and tug vessels (ABP, unpublished).

Temperature within the port ranged from ca. $11^{\circ} \mathrm{C}$ through November to a maximum of ca. $22^{\circ} \mathrm{C}$ during July, average temperature for the entire survey period was ca. $17.5^{\circ} \mathrm{C}$. Temperatures over $22^{\circ} \mathrm{C}$ were sustained for a maximum of three consecutive days through July, with temperatures over $20^{\circ} \mathrm{C}$ sustained from $28^{\text {th }}$ June through to $15^{\text {th }}$ August. Mean salinity was recorded as 28.5 , with no significant stratification. Whilst the Bristol Channel experiences a tidal range of up to $13 \mathrm{~m}$, water levels within the port are maintained at around $10-12 \mathrm{~m}$ through regular pumping directly from the Bristol Channel to replace water lost primarily through lock operation. These docks therefore offer a unique insight into an isolated subtidal habitat which is influenced by water from the Bristol Channel as well as any potential species introductions through maritime trade or aquaculture. 


\subsubsection{Site selection}

A total of 3 sites were selected for the deployment of survey materials, called Zone A, B and C in this study (Figure 5). Due to the level of activity within the Port of Swansea, safe operation was a key factor in identifying suitable deployment sites. Sites were selected based on a) the availability of surface mounting points (e.g. mooring bollards, fences, shackles etc.), b) the proximity to active working berths or derelict infrastructure for safety reasons and to minimise the chance of removal of materials, c) the proximity to other sites to ensure a wide coverage across the docks. Each zone containing two deployment sites, one for each distinct survey type, located within close proximity of one another $(<10 \mathrm{~m})$. Zone A did not meet this criterion due to the busy operational quay limiting safely accessible mounting points; mixed material survey materials for this zone were hence deployed at the nearest suitable location (Figure 5). Zones B and C do contain two deployment sites located within $<10 \mathrm{~m}$ of each other.

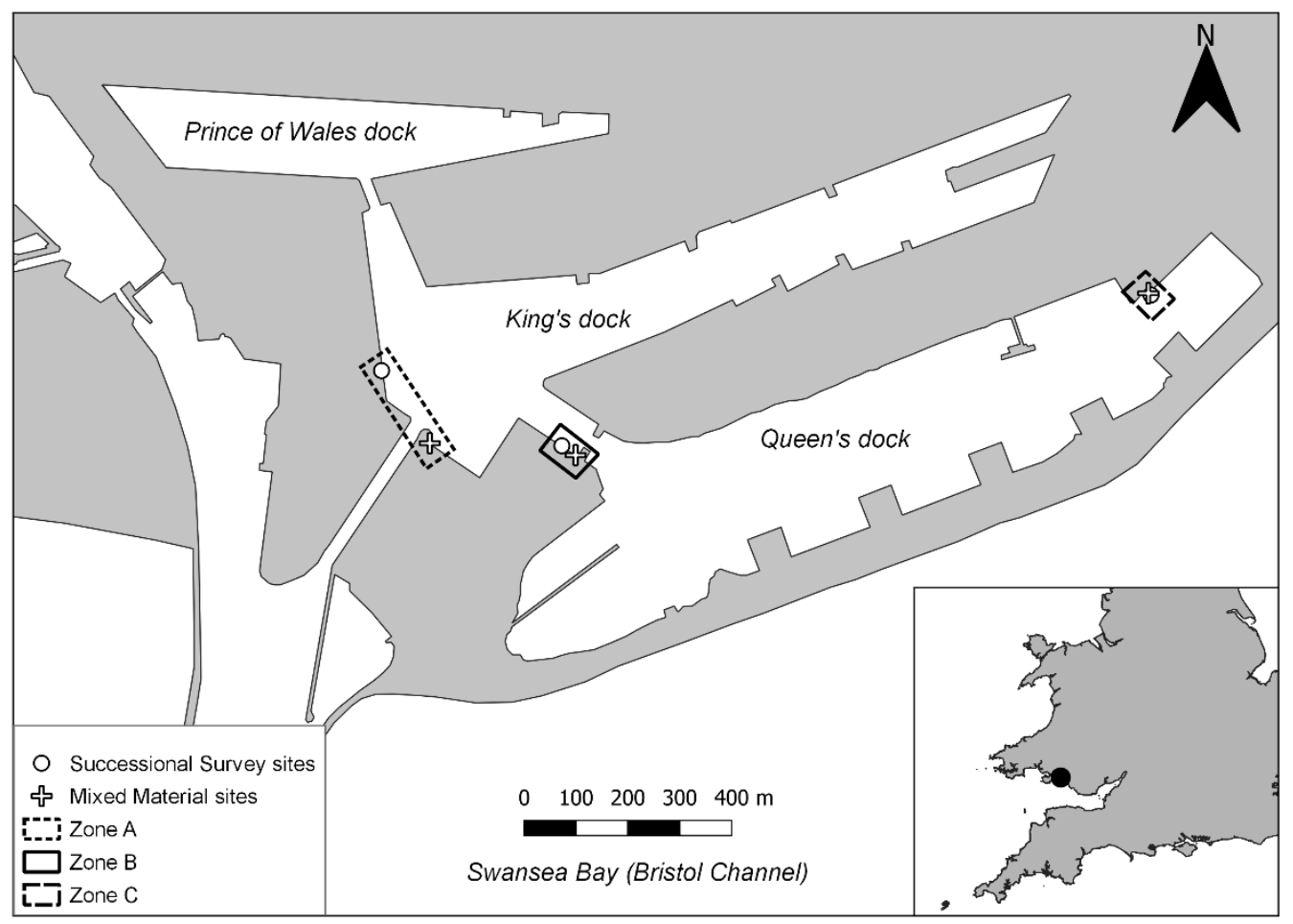

Figure 5. Map outlining the position of survey sites within the Port of Swansea. Each zone contains a location for the deployment of both a 'Successional Settlement Survey' and a 'Mixed Material Survey'. Zones are outlined showing paired sites. 


\subsubsection{Successional Settlement Survey (SSS)}

Acrylic (PMMA) tiles $\left(225 \mathrm{~cm}^{2}, 15 \mathrm{~cm} \mathrm{x} 15 \mathrm{~cm}\right.$, grey in colour) were used as the settlement material. 6 tiles per site were lightly sanded using 40 grit sandpaper and mounted, using cable ties, within an aluminium frame (Figure 6). Each frame was suspended in the water column using polypropylene rope, attached to a fixed surface mounting point (e.g. a mooring bollard; Figure 6). Frames were suspended initially to a depth of approximately $4 \mathrm{~m}$, although the water level in the port can vary meaning that depth did not remain constant during deployment. The rear of tiles (facing into the port wall) sat against the port wall but the whole frame was capable of moving away from the port wall depending on water flow. Materials remained in deployment for 6 months from deployment in May 2018 until collection in November 2018.

This survey type was designed to provide quantified measures of certain ecological parameters, namely species abundance and percentage cover, as well as informing on the colonisation rate and whether there is a successional change in community assemblage over the deployment period. Mounting six tiles within one frame also overcame some of the logistical issues of working within an active port, most notably the lack of availability of safe working areas.

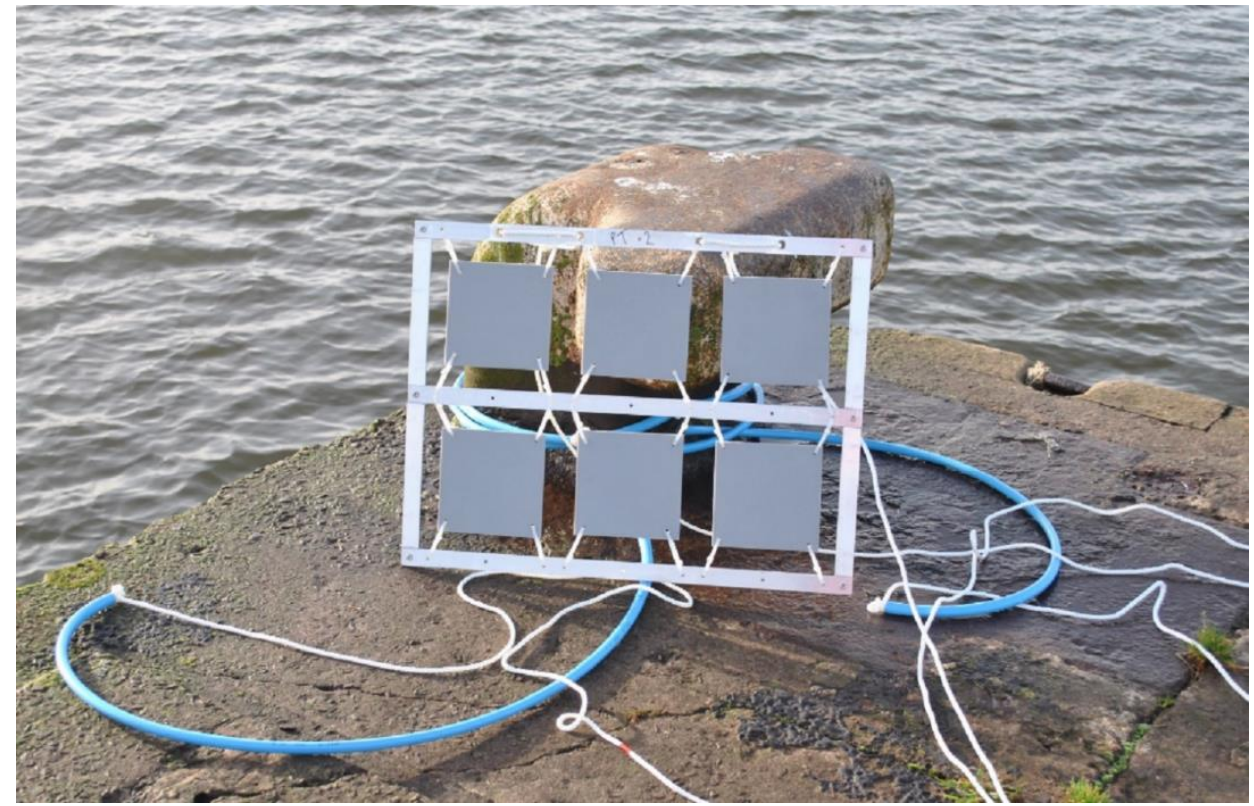

Figure 6. Successional Settlement Survey materials prior to deployment. 


\subsubsection{Mixed Material Survey (MMS)}

This survey type comprised various materials that are commonplace in most ports, each acting as a settlement surface for larval settlement and development. Materials included brick, soft wood (constructional timber, pressure treated), rope (natural fibre and polypropylene), steel, plastic (acrylic tiles as used in the Successional Settlement Survey, and PVC tubing), and a cotton fibre mop head (to represent more complex fibrous materials). Both forms of plastic included sanded and un-sanded variations to investigate any potential settlement preferences based on material roughness. Materials were connected in a set sequence along lengths of rope (Figure 7) and, as with the Successional Settlement Survey, suspended in the water from a fixed surface mounting point. Depth of deployment ranged from approximately $3-6 \mathrm{~m}$, based on the length of the materials and fluctuations in water level. Materials were deployed for 8 months from May 2018 to early February 2019. This survey was designed to investigate whether there is any material preference for settlement of organisms, non-native or native, and whether community composition varied between materials.

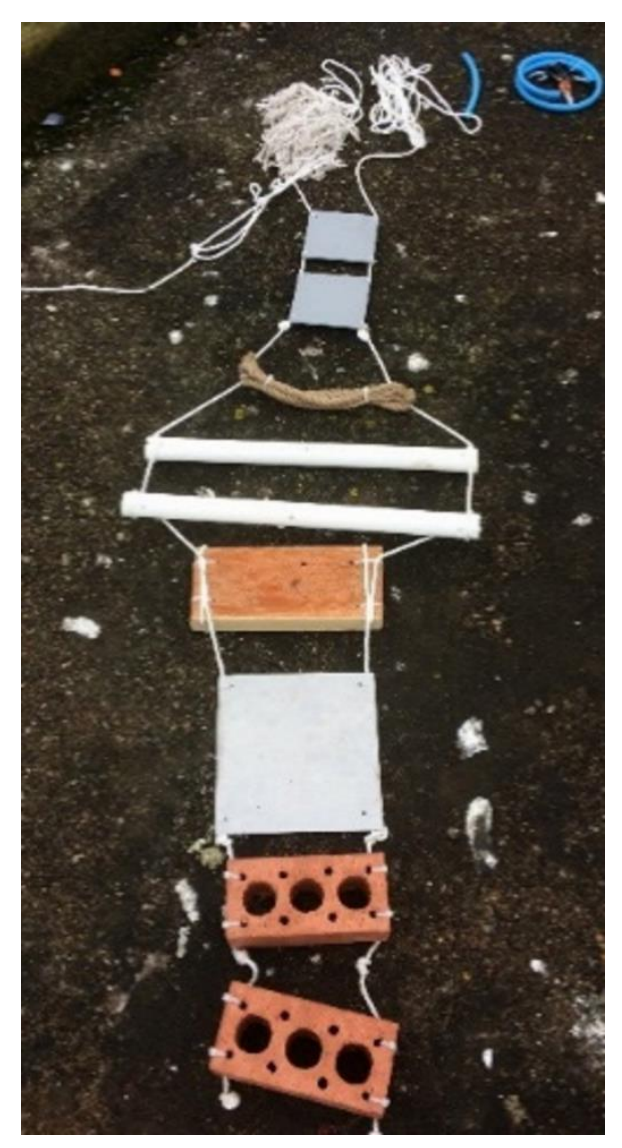

Figure 7. Mixed Material Survey materials prior to deployment. 


\subsubsection{Sampling}

Materials for each survey type were deployed during May 2018 at three sites (Zones) within the Port of Swansea (Figure 5). Zones were visited monthly over a deployment period of 6 months and 8 months for the SSS and MMS, respectively. For the SSS, one acrylic tile was removed from the frame each month and taken for laboratory-based taxonomic identification of the species present. MMS materials remained untouched throughout deployment. Materials were collected after 8 months, following a detailed description of colonisation and identification of species in the field. Motile fauna were consistently recorded if found on a settlement tile at the time of sampling, despite the fact that these organisms could freely move between tiles.

\subsubsection{Laboratory analysis}

Samples collected as part of the SSS underwent laboratory-based analysis. Acrylic tiles were destructively sampled, whereby organisms were systematically removed and identified from both sides of the settlement tiles. Analysis consisted of a visual taxonomic identification to the lowest possible taxon of macrofauna present on tiles and in scrape samples. A combination of dissection and compound microscopes were used where necessary, and identification of species was achieved with the aid of various guides including 'Handbook Of The Marine Fauna Of NorthWest Europe' (Hayward and Ryland, 2017), 'British Marine Amphipoda' (Lincoln, 1979) and Linnean Society taxonomic materials. When necessary, organisms (e.g. amphipods and polychaetes) were fixed and preserved for short periods of time in $70 \%$ ethanol to aid identification.

\subsubsection{Data analysis}

\subsubsection{Percentage cover analysis}

Percentage cover of PMMA tiles (SSS and MMS) and selected other materials forming the MMS (brick, PVC pipe, steel and wood) was calculated from photos, using ImageJ software. Images were set to a known scale and covered areas were measured, with percentage calculated using a known total surface area of materials. 
Three materials (mop head, polypropylene rope, sisal rope) were omitted from the percentage cover analysis due to the inaccuracies in being able to measure coverage. For the SSS only, percentage cover was recorded for both the front (facing into the water column) and rear (facing into the port wall) orientation of settlement tiles. All statistical analyses in this section were performed within RStudio v.1.2.1335 (R Core Team, 2017).

Percentage data for both survey types was converted to proportion (range 0 1) before any statistical analysis. Data from the SSS were found to be non-normally distributed based on Shapiro-Wilk normality tests $(\mathrm{p}<0.05)$ for both proportion and arcsine transformed proportion data. A beta regression was used to statistically analyse the effect of 'Month' and 'Orientation' on the observed percentage cover. Post-hoc Mann-Whitney U tests were used to analyse the pairwise differences between month groups.

As with the SSS, proportion data recorded within the MMS were found to be non-normally distributed based on Shapiro-Wilk tests of both proportion and arcsine transformed data $(\mathrm{p}<0.05)$. A Kruskal-Wallis test was used here to analyse the effect of Material on the observed percentage cover. Dunn's Tests were performed as post-hoc pairwise analyses between material groups.

\subsubsection{Whole community analysis}

Primer 6 v.6.1.13 with PERMANOVA v.1.0.3 software (Anderson et al., 2008; PERMANOVA+ for PRIMER software) was used to analyse whole community abundance data between samples collected within the SSS and within the MMS. Four species identified could only be recorded by measure of area covered $\left(\mathrm{cm}^{2}\right)$, rather than abundance counts, and these remained within the analysis. Data were first transformed using a $\log (\mathrm{x}+1)$ transformation. This transformation was selected in order to downweigh a small number of highly abundant species, thus increasing the importance of species diversity within the analyses, as well as to accommodate for the combined use of abundance and coverage data within the same analyses. 
The Bray Curtis similarity index was used to create a similarity matrix, from which non-metric multidimensional scaling (nMDS) and PERMANOVA analyses were performed. PERMANOVAs were designed with two factors (Zone and Month for the SSS, and Zone and Material for the MMS) and one response variable (values in the similarity matrix). No interaction term between factors was included. The model used the permutation of residuals under a reduced model with type III (partial) sum of squares and 9999 permutations. Pairwise PERMANOVAs were used ad hoc to analyse the differences between certain factor groups (Anderson et al., 2008). Factor groups were Zone: A, B, C; Material: Brick, Mop, PMMA (sanded), PMMA (unsanded), Polypropylene rope, PVC (sanded), PVC (unsanded), Sisal rope, Steel, Wood.

\subsubsection{Cross survey analysis}

Species richness data (as total number of species recorded per sample, irrespective of surface area) was used in cross-survey analyses. These analyses were conducted to evaluate the effectiveness of each survey type at describing the whole fouling community, as well as identifying NNS. A Fisher-Pitman permutation test (Berry et al., 2002) was conducted within R v. 3.6.2 (R Core Team, 2017), whereby the effect of the factor Survey Type (2 levels: SSS, MMS) on the response variable species richness was analysed.

\subsection{Results}

\subsubsection{Successional Settlement Survey (SSS)}

A total of 40 different taxa across 9 phyla were identified as part of the SSS (Supplementary Table S1). Of these, 7 may be classified as non-native species (NNS) within the UK. Arthropoda was the most represented phylum with 13 different species, whilst only one species each of Echinodermata, Platyhelminthes and Porifera were identified. In terms of total abundance and coverage Chordata was the most common phylum, of which all but one of the species were within the class Ascidiacea. A total of 1264 individuals of Ciona intestinalis were recorded over the 6-month survey period, making this the most abundant species. Sampling photos available in Appendix III. 
The similarity in communities among zones and months were visualised by nMDS (Figure 8). Both factors, Zone and Month, significantly affected the structure of the faunal communities; 'Zone' (PERMANOVA, pseudo-F $=3.19, \mathrm{p}=0.0029$ ) and 'Month' (PERMANOVA, pseudo-F $=5.05, \mathrm{p}=0.0003$ ). Pairwise tests among Zones showed a significant difference in community assemblage between Zone $\mathrm{C}$ and Zone A (PERMANOVA, $\mathrm{t}=1.411, \mathrm{p}=0.0299)$.

The total number of species recorded per sample increased consistently to a maximum mean of 17.33 species per sample in October before falling to 14 species per sample in November (Figure 9). Ascidiella scabra was amongst the first organisms to begin colonisation in July and rapidly increased in abundance to a peak mean of 82.67 individuals per sample in September before falling through October and November. The abundance of amphipods declined at a similar time to A. scabra. A similar downward trend from September through to November can be seen in the total number of non-native species (NNS) recorded per sample, from a maximum mean of 3.67 species per sample in September to 2.33 species per sample in November. Conversely, the coverage of colonial ascidians and the abundance of Aurelia aurita polyps began to increase from September through to a maximum recorded mean coverage in November of $6.33 \mathrm{~cm}^{2}$ per sample for colonial ascidians and mean abundance of 50 individuals per sample for A. aurita polyps. 


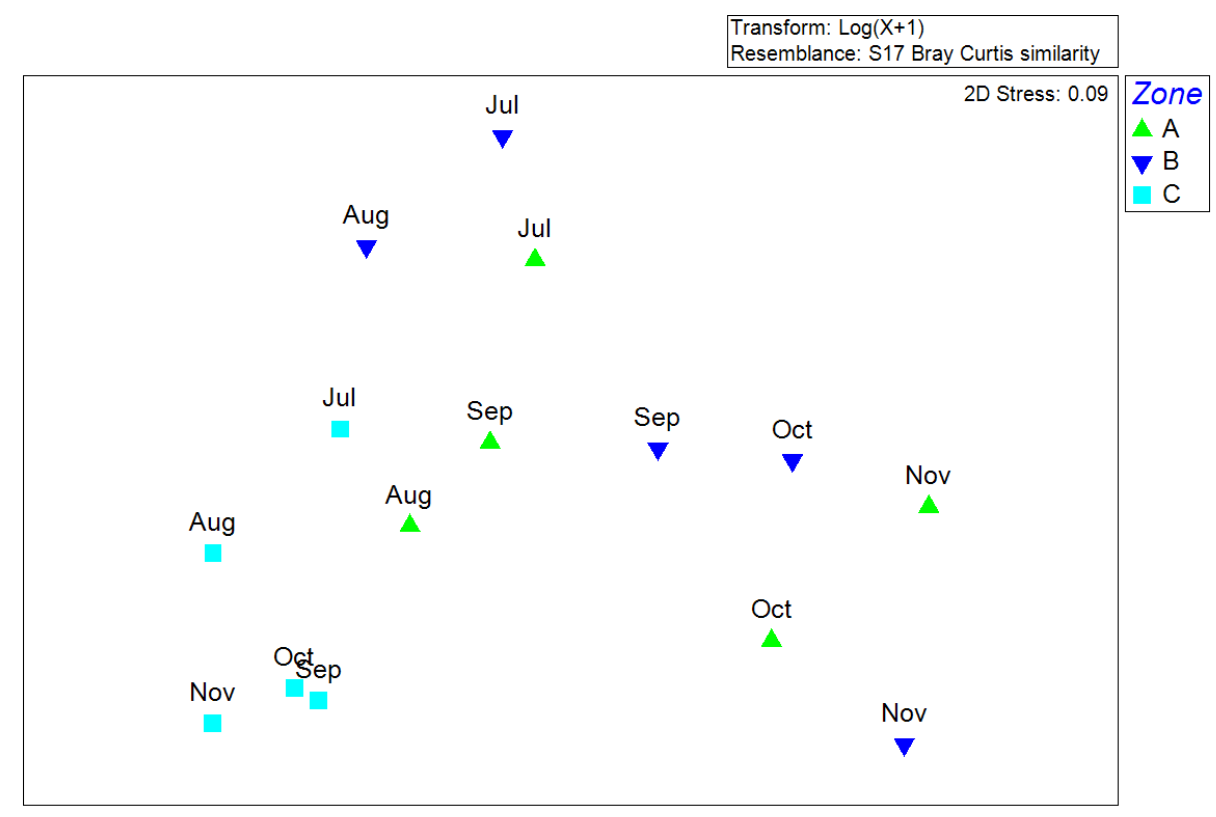

Figure 8. nMDS plot of colonising species communities collected within the Successional Settlement Survey. Plot based on a resemblance matrix created using a Bray Curtis similarity indices of $\log (\mathrm{X}+1)$ transformed abundance data. Samples labelled by factor 'Month'; symbols represent location factor 'Zone'; June samples were removed from plot since communities were so species-poor that they could not be plotted in a meaningful way in relation to subsequent months.
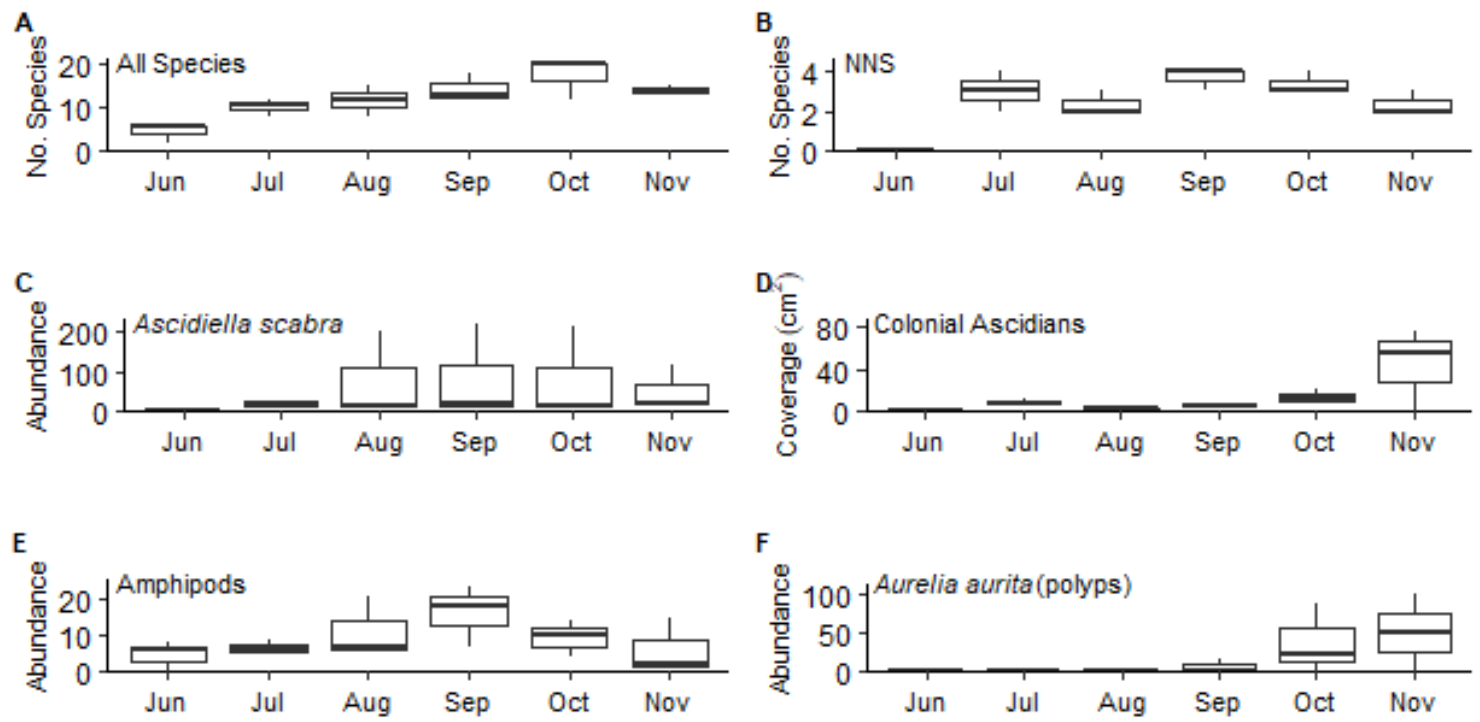

Figure 9. Colonisation of settlement tiles in Swansea Port $\left(450 \mathrm{~cm}^{2}, \mathrm{n}=3\right)$. A: total number of species recorded. B: number of non-native species (NNS). C: abundance count of Ascidiella scabra. D: surface area coverage $\left(\mathrm{cm}^{2}\right)$ of colonial ascidians. E: abundance count of amphipods. F: abundance count of Aurelia aurita polyps. 
Percentage cover was recorded for both the front (facing away from the port wall) and the rear (facing towards the port wall) of each tile each month (Figure 10). Two months after deployment colonisation reached over $90 \%$ and $70 \%$ coverage for the front and rear of tiles, respectively. Coverage of the front of tiles remained over $90 \%$ for the reminder of the survey period. The rear orientation of tiles took until September to reach ca. $90 \%$ coverage, with the maximum coverage of $97 \%$ being achieved in October. There is no evidence here that the factors 'Month' (Beta regression, $\mathrm{z}=1.399, \mathrm{p}=0.162$ ) and 'Orientation' (Beta regression, $\mathrm{z}=-0.579, \mathrm{p}=$ 0.563 ), nor the interaction of these factors (Beta regression, $z=0.212, p=0.832$ ) significantly affect percentage cover. Pairwise analyses between months showed that percentage cover increased significantly from June to September (Mann-Whitney U test, $\mathrm{p}=0.0449$ ), June to October (Mann-Whitney $\mathrm{U}$ test, $\mathrm{p}=0.0081$ ), and June to November (Mann-Whitney U test, $\mathrm{p}=0.0043$ ); percentage cover was not significantly different between any other month groups (Mann-Whitney U tests, $p>$ $0.05)$.

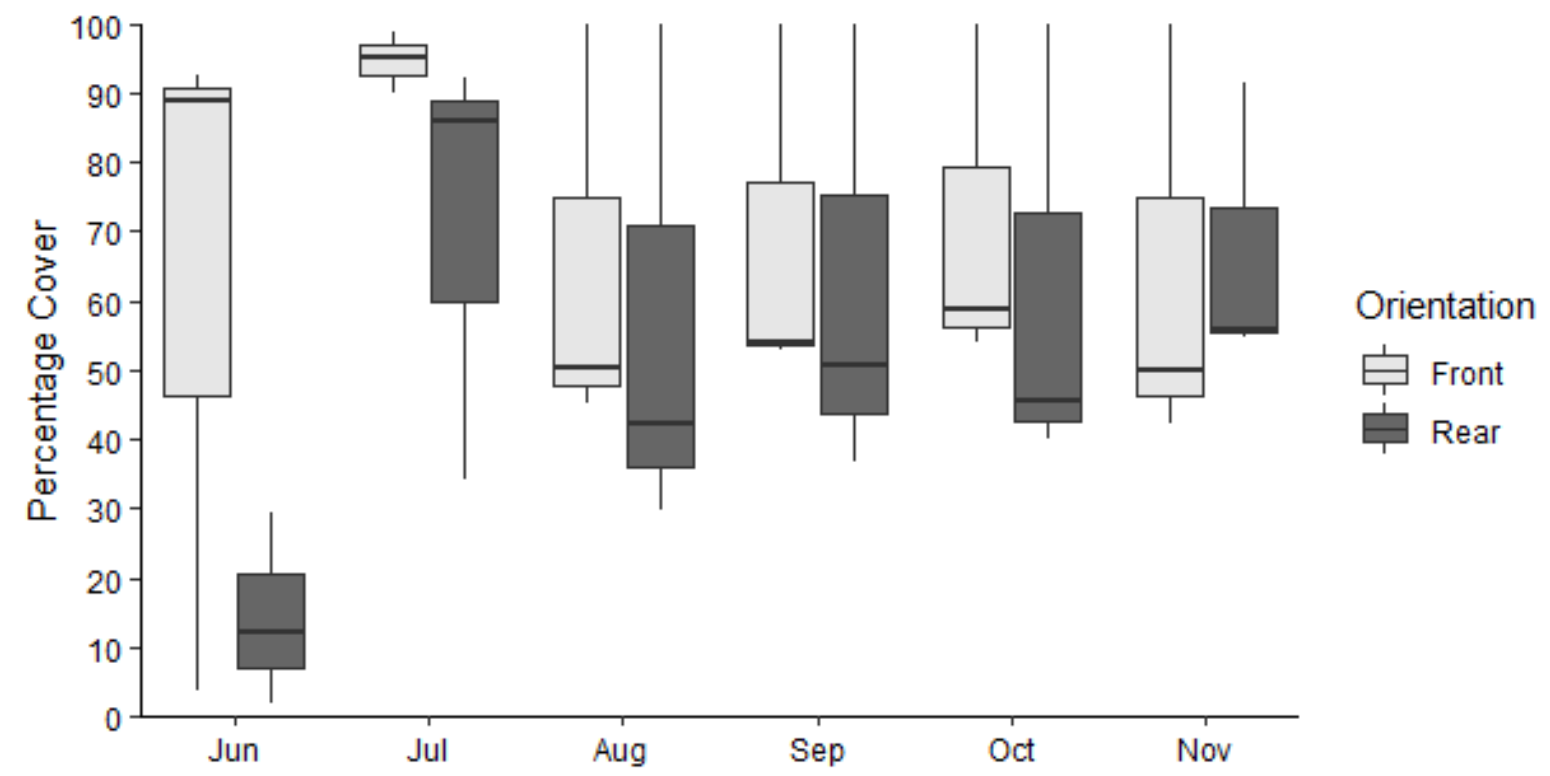

Figure 10. Percentage cover of PMMA tiles recorded each month within the Successional Settlement Survey $\left(225 \mathrm{~cm}^{2}, \mathrm{n}=3\right)$. 'Front' refers to the orientation of tiles facing away from port walls, 'Rear' refers to the orientation of tiles facing the port walls. 


\subsubsection{Mixed Material Survey (MMS)}

15 species were recorded within the MMS surveys (Supplementary Table S2). As with the SSS, Arthropoda was the most represented phylum with 7 species. Species with the highest total abundances were within the phylum Chordata, the most abundant species here being the Ascidiacea Ascidiella scabra and Ciona intestinalis with a total recorded abundance of 264 and 337, respectively, across all materials and all zones (Supplementary Table S2). Several species were recorded from only one material: Spirobranchus triqueter and Palaemon serratus from sanded PMMA tiles, Carcinus maenas and Macropodia rostrata from mop heads and Bugula neritina from unsanded PMMA tiles.

Community analysis indicated that both material type (PERMANOVA, pseudo-F $=2.57, \mathrm{p}=0.0011)$ and zone (PERMANOVA, pseudo-F $=3.44, \mathrm{p}=$ 0.0008) significantly influenced the colonising fauna (Figure 11). However, there was no apparent grouping of material types, except for sisal rope due to the presence of only one organism, Ciona intestinalis, on the material. Pairwise comparison of all materials did not identify significant differences between isolated materials (PERMANOVA, $\mathrm{p}>0.05$; due to a small sample size of three within each material group the number of permutations completed was below the required level to consider the analysis reliable). Samples from Zone $\mathrm{C}$ were clustered together and pairwise analysis showed a significant difference between the communities within zones A and C (PERMANOVA, $\mathrm{t}=1.411, \mathrm{p}=0.033)$. All other pairwise comparisons were not significant. 


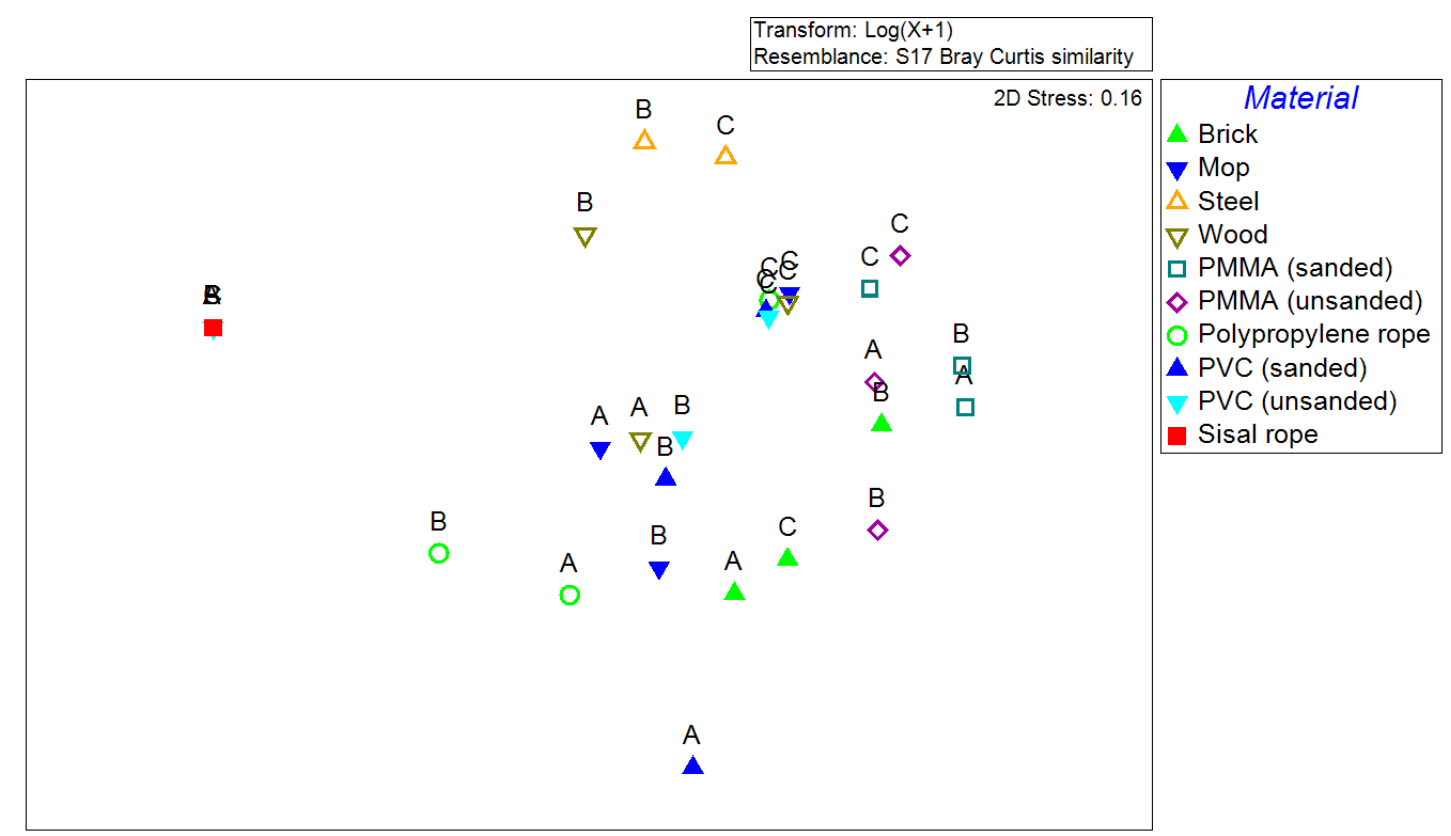

Figure 11. nMDS plot of samples collected within the MMS. Raw data transformed using a $\log (\mathrm{X}+1)$ transformation. Plot based on a resemblance matrix created using a Bray Curtis similarity index. Samples labelled by factor 'Zone'; symbols represent factor 'Material'.

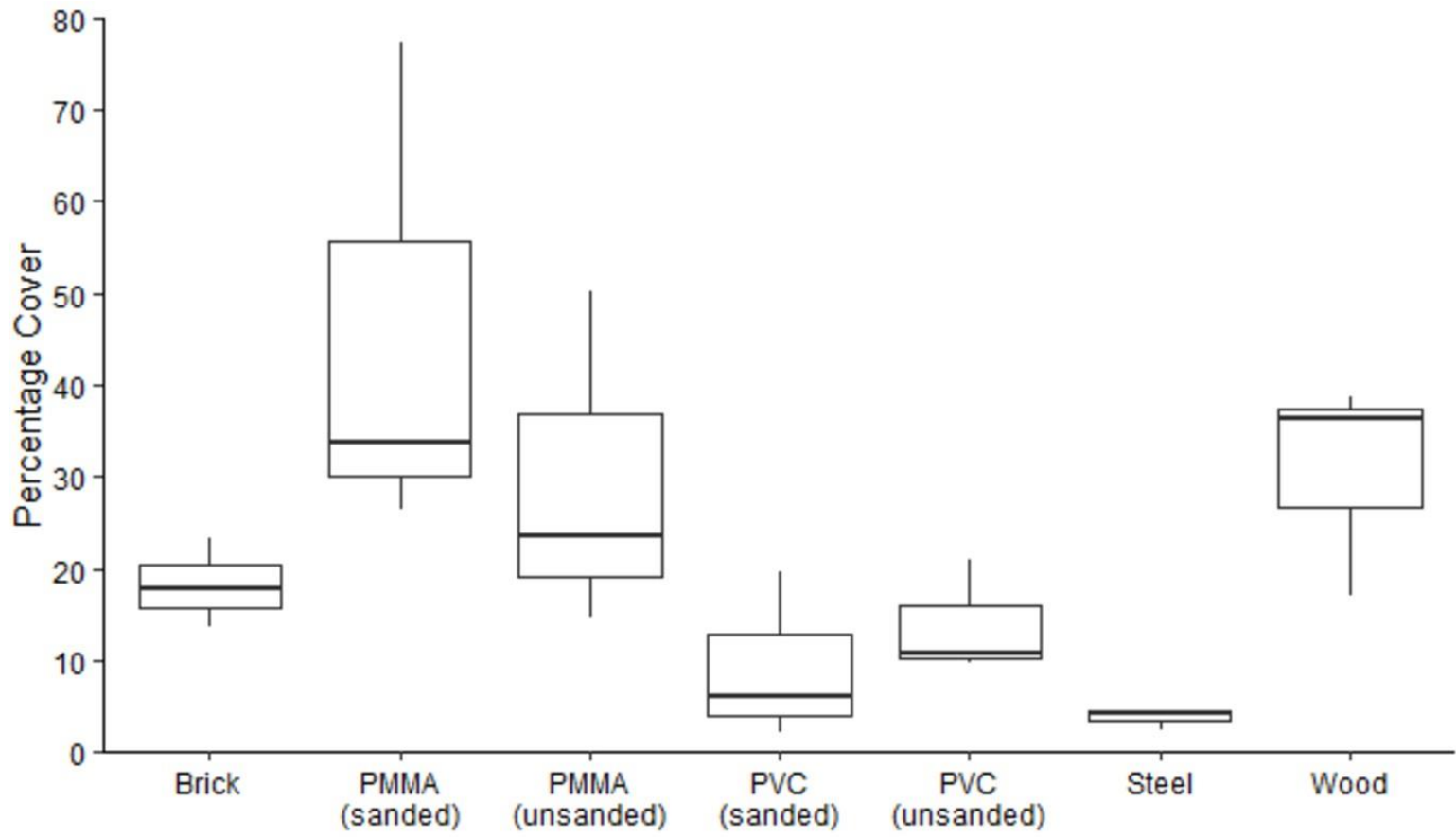

Figure 12. Total percentage cover of all organisms present on selected materials from within the MMS after eight months. $\mathrm{n}=3$ for each material. 
Seven of the ten materials present within the MMS had the total colonised area measured and converted to percentage cover (Figure 12). Each form of PMMA tile (sanded and unsanded) along with wood were among the most heavily colonised materials. Sanded PMMA and wood had the highest median coverage of $34 \%$ and $36 \%$, respectively, after eight months of deployment. Steel was consistently recorded with the smallest amount of colonisation with a median of $4 \%$. Material type was found to have a significant effect on the observed percentage cover (Kruskal-Wallis, $\mathrm{p}=0.031$ ). Significant differences in percentage cover were identified between: sanded PMMA and sanded PVC; sanded PMMA and steel; unsanded PMMA and steel; wood and steel (Dunn's Test, $\mathrm{p}<\alpha / 2$ where $\alpha=0.05$ ). No significant differences in percentage cover were recorded between all other material pairs (Dunn's Test, $p>\alpha / 2$ where $\alpha=0.05$ ).

The total abundance of organisms recorded from each material was standardised by surface area to abundance counts per $500 \mathrm{~cm}^{2}$ (Figure 13). PMMA tiles had the greatest abundance of organisms per $500 \mathrm{~cm}^{2}$ with median values of 130 (sanded PMMA) and 56.7 (unsanded PMMA). The mop head saw the lowest abundance per $500 \mathrm{~cm}^{2}$ with 1.4. However, the copious strands of the mop head had a far greater surface area than any other material $\left(6960 \mathrm{~cm}^{2}\right)$; on average $29.5 \pm 25.0 \mathrm{sd}$ organisms were recorded per mophead. Material type significantly influenced the abundance of organisms per $500 \mathrm{~cm}^{2}$ surface area (Kruskal-Wallis, $\mathrm{p}=0.01$ ). Pairwise tests revealed significant differences between the following material types: sanded PMMA and mop head, sanded PMMA and polypropylene rope, sanded PMMA and sisal rope, sanded PMMA and steel, unsanded PMMA and mop head, unsanded PMMA and sisal rope, unsanded PMMA and steel (Dunn's Test, $p<\alpha / 2$ where $\alpha=0.05)$. No significant differences in the abundance per $500 \mathrm{~cm}^{2}$ were recorded between any other material pairs (Dunn's Test, $p>\alpha / 2$ where $\alpha=0.05$ ). 


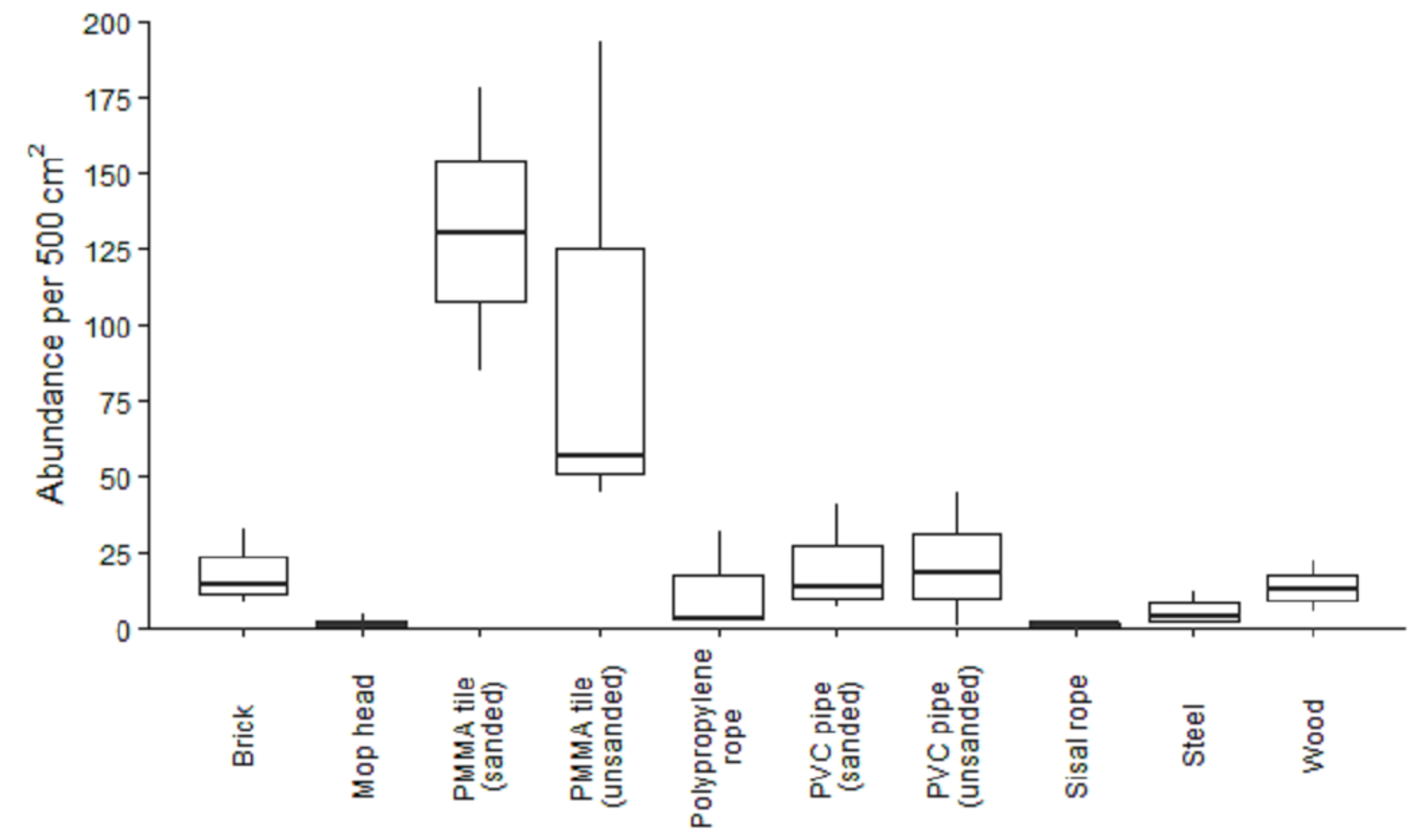

Figure 13. Total abundance of organisms per $500 \mathrm{~cm}^{2}$ recorded on each material used within the MMS. Abundance counts standardised using the surface area of each material to allow for direct comparison. $\mathrm{n}=3$.

\subsubsection{Non-native species (NNS)}

A total of 7 NNS were recorded during this research. All 7 species were identified from within the SSS, with only 5 of the 7 identified within the MMS (Table 1). Caprella mutica and Monocorophium acherusicum were the two species found exclusively within the SSS. Bugulina stolonifera had the greatest average abundance of all NNS in the SSS as well as MMS, and a second bryozoan, Bugula neritina, had the lowest average abundance. 
Table 1. Mean abundance of NNS per $500 \mathrm{~cm}^{2}( \pm$ standard error) of each NNS recorded within the two survey types: Successional Settlement Survey (SSS) and Mixed Material Survey (MMS).

\begin{tabular}{lrr}
\hline \multirow{2}{*}{ NNS } & \multicolumn{2}{c}{ Survey Type } \\
\cline { 2 - 3 } & \multicolumn{1}{c}{ SSS } & \multicolumn{1}{c}{ MMS } \\
\hline Austrominius modestus & $13.7 \pm 6.9$ & $72.3 \pm 49.9$ \\
Brachynotus sexdentatus & $0.3 \pm 0.3$ & $0.7 \pm 0.3$ \\
Bugula neritina & $1.0 \pm 1.0$ & $0.7 \pm 0.7$ \\
Bugulina stolonifera & $115.3 \pm 20.1$ & $74 \pm 50.6$ \\
Caprella mutica & $18.0 \pm 10.0$ & - \\
Monocorophium acherusicum & $5.3 \pm 2.4$ & - \\
Styela clava & $3.0 \pm 3.0$ & $8.3 \pm 5.8$ \\
\hline
\end{tabular}

\subsubsection{Cross-Survey Analysis}

Species richness data (as the total number of species recorded in samples) were analysed across the two survey types to identify the effectiveness of each survey type at describing fouling communities as well as identifying NNS. Considering all species, the SSS attracted a larger number of species compared with the MMS (Figure 14). This difference was found to be statistically significant (Fisher-Pitman permutation test, $\mathrm{Z}=-2.0207, \mathrm{p}=0.0433$ ) and therefore demonstrates that survey type was a significant factor in determining the number of species recorded. Survey type was found to have no significant effect on the number of NNS recorded (Fisher-Pitman permutation test, $\mathrm{Z}=-0.488, \mathrm{p}=0.6256$ ). 


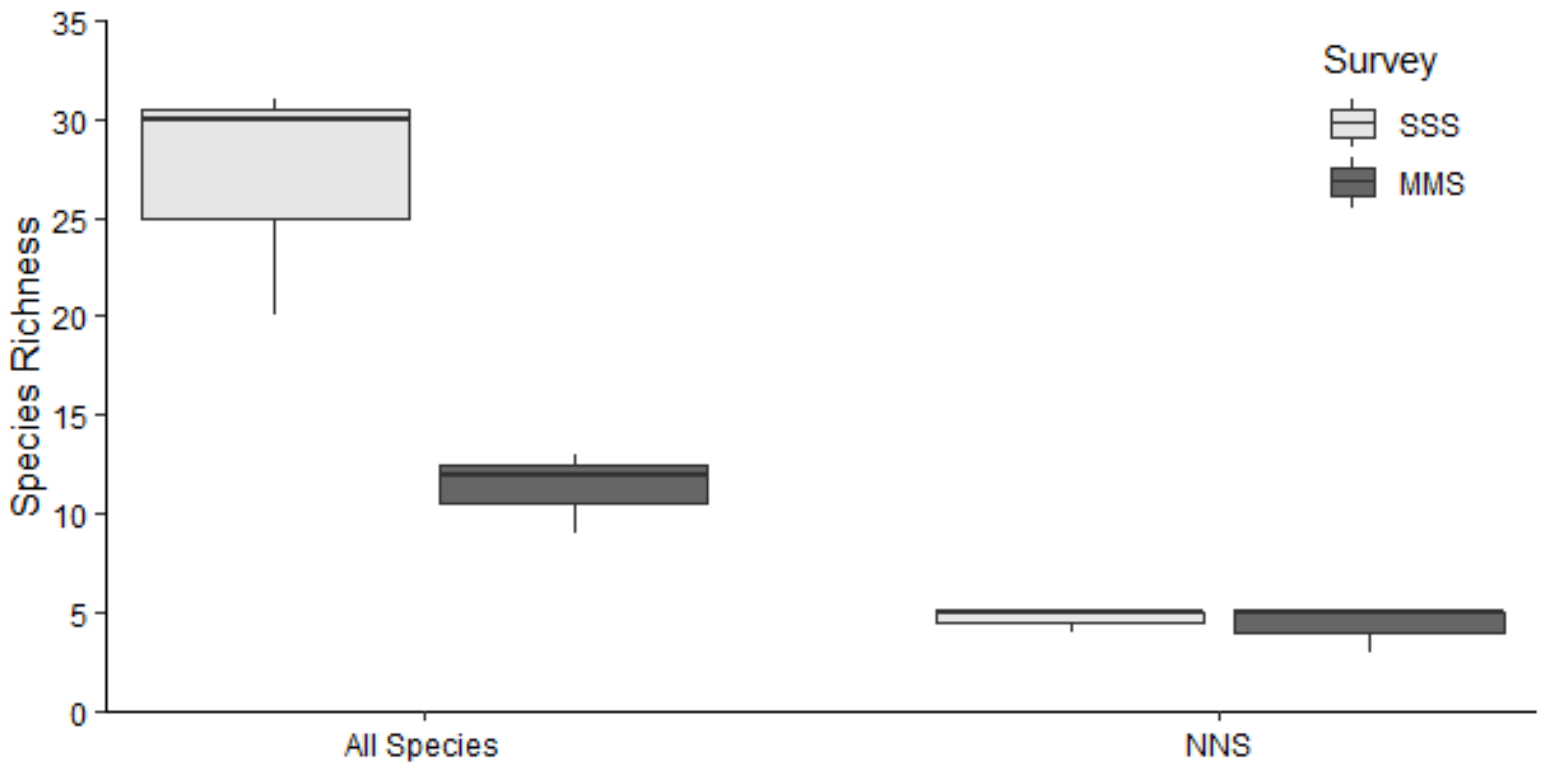

Figure 14. Average species richness recorded with the two survey types 'successional settlement survey' (SSS) and 'mixed material survey' (MMS). Species per $2700 \mathrm{~cm}^{2}$ for SSS (sum of 6 tiles per zone), $13533 \mathrm{~cm}^{2}$ for MMS (sum of all materials per zone); $\mathrm{n}=3$.

\subsection{Discussion}

\subsubsection{Fouling communities}

Selection of materials by sessile benthic organisms is more complex than that of mobile organisms, relying on a wide range of factors including orientation, position, material, light, pollution, recruitment, competition, predation and biofilms, amongst others (Sutherland and Karlson, 1977; Osman, 1977; Harris and Irons, 1982; Keough and Downes, 1982; Glasby, 1999, 2000; Glasby and Connell, 2001; Dobretsov et al., 2005; Blockley and Chapman, 2006; Qian et al., 2007; Tyrrell and Byers, 2007; Nydam and Stachowicz, 2007; Crooks et al., 2011). Applying two different survey types greatly improved our knowledge of the fouling community present within an industrial port environment. The rapid rate of colonisation of settlement tiles within the successional settlement survey (SSS) was striking. Colonisation to over $90 \%$ coverage took only two months for the sides of tiles facing the water column. 
Colonisation during this time period (May through to July) was expected as this coincides with the annual phase of benthic larval settlement, although the process can be highly variable (Keough, 1983; Ronowicz et al., 2014). Colonisation rates were comparable to those observed in a similar study conducted in marinas in North Wales (Bangor University, 2015), even though the study focussed on individual species and did not record overall coverage.

Of the 38 species identified in total, 18 fell into the functional group of filter feeders, including the three most abundant species: Ciona intestinalis, Ascidiella scabra and Bugulina stolonifera. In natural ecosystems filter feeders can play a key role in structuring phytoplankton communities and in nutrient cycling, and therefore controlling primary production to an extent (Stein et al., 1995; Sánchez et al., 2016). Given that the Port of Swansea is an enclosed system, it is likely that filter feeders, particularly the abundant solitary ascidians, play a major role in determining clearance rates and forming the observed communities. Filter feeders have also been identified as keystone species in other systems (Persson et al., 2007), the effect of which may be further enhanced through the production of faecal pellets which may support a range of different organisms such as the detritivores identified within this study (Ostroumov, 2005). It was observed that ascidian species would readily settle directly onto the tests of A. scabra, with up to five different ascidian species being recorded on one individual. A. scabra has a cartilaginous test making it rigid with a rough texture, creating a viable surface for larval settlement. By contrast $C$. intestinalis, the only other frequently abundant and large solitary ascidian identified, has a more flexible and softer test, and consequently no organisms were observed to settle directly onto this species. It follows therefore that A. scabra may be a key species in increasing biodiversity within certain fouling communities.

By removing one settlement tile per month as part of the SSS it was possible to investigate community succession during the first six months of deployment. It provided greater survey power by recording species that were not present on settlement materials after six months but were present for intermediate stages within the six month period, thus providing a more complete insight into fouling communities rather than single 'snapshots' in time as is often the case when using settlement panels (Bangor University, 2015; Cook et al., 2015; Hurst, 2016). 
Community structure was found to change over the course of the survey period, which I suggest here may be influenced by the presence of $A$. scabra. Dense aggregations of $A$. scabra appeared to support a number of additional taxa, such as various amphipod species, through the initial four months of colonisation. This is made more apparent given the fall in the total number of species recorded per sample occurring concurrently with the fall in abundance of $A$. scabra. Succession in this study saw the increase in coverage of colonial ascidians following the decline in $A$. scabra abundance. It is thought that the more two-dimensional habitat created by the colonial ascidians, compared to that created by dense $A$. scabra aggregations, provides less space, shelter and access to food, which are important factors in habitat selection by cryptic organisms (Aikins and Kikuchi, 2001). This resulted in the transition from a more species-rich fouling community, when A. scabra was present in high abundance, to a more species-poor community as colonial ascidians increased in dominance.

Knowledge of how densely different materials are colonised and which species colonise each material can contribute to informing port management plans. This may be, for example, to adopt strategies to increase port biodiversity by focussing more on the types of materials that are present within the port. Community composition, total colonised area and the abundance of organisms per $500 \mathrm{~cm}^{2}$ were all found to be significantly influenced by material type. PMMA in particular was consistently found to support a greater abundance and total colonised area than most other materials, which may have wider implications for the distribution of organisms associated with marine litter (Miralles et al., 2018). Fibrous materials, such as rope and mop heads, were amongst the least colonised materials despite certain species such as $C$. mutica being commonly found on fibrous materials in previous observations (Cook, personal communication). This is thought to be based largely on larval habitat selection preferences, where more solid and secure substrata such as plastic, wood and brick is favoured (Osman, 1977). Steel too would ordinarily be considered a viable substrate for larval settlement. However, accelerated low water corrosion (ALWC; Marty et al., 2014; Smith et al., 2019) was found to impede the colonisation of steel, likely through chemical interaction with larvae or by creating a physical barrier for larval settlement (Smith et al., 2019). 
Contrary to observations made in the MMS, both of the sessile species recorded from only one material, Bugula neritina and Spirobranchus triqueter, have been reported to colonise a wide range of materials in previous studies (Li et al., 2016; Gündoğdu et al., 2017), and indeed in the SSS survey within this study. Whilst it is difficult to directly compare observations made here to previous colonisation studies, particularly ones from different environments, it is probable that a number of the factors listed above are resulting in the reduced colonisation of some materials by certain organisms. Given the high abundance of Ascidiella scabra, Ciona intestinalis and Bugulina stolonifera recorded in this survey across most materials, it seems likely that competition for space and food would be the primary factor in limiting colonisation by various other species. Competition is likely enforced by a lack of larval recruitment, particularly with $B$. neritina as this species was recorded only five times across both survey types, which suggests there may be low larval recruitment for this species within the port. This information could be applied by port operators to promote certain communities within ports, or to increase the efficiency of port activities and processes by using specific material types.

\subsubsection{Non-native species (NNS)}

A total of 7 non-native species (NNS) were identified and recorded as part of this study, with only one species, Brachynotus sexdentatus, not being considered 'established' within the UK (NBN Atlas, 2019). The date of first record for these species range from as long ago as 1875 for Bugulina stolonifera (Ryland, 1960) to as recent as 2000 for Caprella mutica (Willis et al., 2004). Each of the established NNS can be found at various locations around the UK, having spread beyond the site of first introduction (Ryland, 1960; Eno et al., 1997; Bracewell et al., 2012).

More locally to Swansea, all but $C$. mutica have been reported from the South Wales coastline with B. stolonifera, Bugula neritina and Brachynotus sexdentatus having been recorded from within the Port of Swansea in the late 1950s (Naylor, 1957). B. sexdentatus has in fact only ever been recorded in the UK from within the Port of Swansea and, along with B. neritina, was thought to have been naturally eradicated from the port following the closure of the Tir John power station in the 1970s (Eno et al., 1997; Arenas et al., 2006). 
Water within the port had been artificially heated whilst the power station was in operation, through the discharge of heated effluent, creating a suitable habitat for the warmer water natives B. sexdentatus and B. neritina (Keough and Chernoff, 1987; Cuesta et al., 2000). Arenas et al. (2006) reported the presence of $B$. neritina from various locations around the UK, in contradiction to Eno et al. (1997), although no surveys were conducted within the Port of Swansea. It is likely that each of these species remained within the port through a successfully reproducing population rather than being reintroduced. It would appear though that the abundance of each species within the port has reduced since the last comprehensive survey was completed in the 1950s (Naylor, 1957). This may well be due to the cooling of the dock water which may have shifted the competitive edge back to some of the native species or indeed NNS, such as B. stolonifera, which have been recorded in high abundance within this study.

It is difficult to accurately comment on how, or even when, these NNS may have been first introduced to the port due to the lack of baseline data. Given the port activities it seems plausible that shipping is the likely pathway for all non-native introductions here. Ports are widely regarded as potential vectors for NNS, where they may be first introduced to a region within a port before spreading more locally along a natural coastline (Bailey, 2015). Regarding the NNS recorded in this study, only $B$. sexdentatus and $C$. mutica have not been previously recorded from elsewhere within the South Wales region. Therefore, these two species are at risk of being dispersed from the port into the natural environment of the Bristol Channel. Whilst it is unknown exactly when $C$. mutica was first introduced to the Port of Swansea, it can, however, be assumed that if the habitats and environmental conditions in the Bristol Channel were suitable for $C$. mutica then it would by now have spread out of the port. C. mutica is a common NNS worldwide and is regularly reported from within ports and marinas (Ashton et al., 2007a). These reports along with experimental studies indicate that $C$. mutica can survive in temperatures ranging from $-1.8^{\circ} \mathrm{C}$ to $25^{\circ} \mathrm{C}$ (Schevchenko et al., 2004) but would likely not survive prolonged exposure to salinities below 18 (Ashton et al., 2007b). Temperature ranges within in the Bristol Channel fall comfortably within the tolerance of $C$. mutica, however the tidal nature of this region can result in salinities of 17 on low tides (Henderson et al., 2012). 
Whilst the lower range of salinities here are short lived, it could potentially be the reason that $C$. mutica has not been recorded from the Bristol Channel. Competition from native caprellid amphipods (Shucksmith et al., 2009) such as $C$. linearis, which is present in South Wales (NBN Atlas, 2019), or the possibility that $C$. mutica is in fact present in the Bristol Channel but has either not been observed in surveys or the data have not been reported in the public domain, may also be reasons for the perceived absence of $C$. mutica in the Bristol Channel.

A lot less is known about $B$. sexdentatus. Whilst temperatures within the Port of Swansea can range from approximately $5^{\circ} \mathrm{C}$ to $25^{\circ} \mathrm{C}$ (unpublished), it is unlikely that $B$. sexdentatus would be able to tolerate the harsh conditions of large tidal range and temperature and salinity fluctuations that define habitats within the Bristol Channel, given that it is native to Southern Europe. The remaining five NNS have been previously recorded from the South Wales region (NBN Atlas, 2019) therefore the current risk of non-native invasion beyond the port is minimal for these species. Port-to-port and port-to-marina transport of NNS remains a risk through either ballast water or hull fouling and should therefore be considered in the port biosecurity risk management protocols. It is, unfortunately, impossible to say whether the NNS appeared first within the Port of Swansea or elsewhere within the Bristol Channel.

\subsubsection{Effectiveness of survey methods}

When discussing the effectiveness of each survey type designed for this study, the success of overcoming specific challenges of surveying within active ports must be considered, as well as the effectiveness of identifying species (both native and non-native), the quality of data provided and some of the more logistical aspects such as cost and level of required expertise.

Previous methodologies for the study of fouling communities in ports describe the use of rapid assessment surveys (RAS; Cohen et al., 2005; Arenas et al., 2006; Rohde et al., 2017), settlement surveys (Tyrrell and Byers, 2007; Floerl et al., 2012; Ronowicz et al., 2014) or a combination of the two (HELCOM, 2013; Cook et al., 2015; Hurst, 2016). 
The SSS described in this study is a development of more conventional settlement surveys whilst the MMS is, in effect, an adaption of a RAS, whereby materials are assembled and deployed rather than using materials that already exist in the port. This aims to overcome the problem of a lack of existing materials that can be safely accessed, which is the primary difficulty of using RAS within ports. The materials within the MMS would need to be in deployment for a number of years to be directly comparable to RAS, which may be considered for longer-term monitoring. Both survey types successfully overcame the primary limitations to conducting fouling community surveys within ports. All survey materials deployed in May 2018 remained undisturbed and were successfully retrieved in the winter of 2018 . No materials interfered with port operations and researchers were able to comfortably work within the port health and safety regulations. This success was due in a large part to extensive field site visits and discussion with port authorities during the planning phase to limit the risk of interference in port activities and potential removal of materials.

As a direct comparison between survey types, the SSS was more effective than the MMS at identifying species, with an average of 27 species present per sample in the SSS compared to only 11 in the MMS. Both survey types did, however, record a similar number of NNS per sample, although two NNS (Caprella mutica and Monocorophium acherusicum) were recorded only from within the SSS. This indicates two key points: firstly, the MMS appears to attract a greater proportion of NNS per sample than the SSS; and secondly that the SSS is more successful at describing whole fouling communities. It could be argued then that there is no need to deploy the MMS, as the SSS offers more in terms of data on fouling communities and can identify more NNS. The MMS does though offer important insight for stakeholders in terms of biosecurity planning. Clearly, a combination of the SSS and MMS provides the most useful data with consideration not only of what species are inhabiting the Port of Swansea, but also which materials may be of interest for future management. 
Since the materials used in each survey were deployed as accurately as possible to a set depth and with the slight fluctuation in the port water level (between $10-12 \mathrm{~m}$ ) over the course of deployment, depth effects may have influenced the observed fouling communities both between survey types and within the MMS, where materials extended over a couple of metres in sequence. Depth is known to be a factor in determining the formation of biofilms and settlement of some organisms (Hurlbut, 1991; Head et al., 2004; Kazmi et al., 2020) and has been found to be a significant factor in determining the community composition in a previous study (Lezzi and Giangrande, 2018). Accommodating multiple SSS frames in sequence at set depths, similar to that done by Lezzi and Giangrande (2018), would enable depth as a factor to be investigated without the need for additional deployment sites. However, weight should be considered if doing this in future and retrieval of survey materials may not be feasible without mechanical assistance during months of peak colonisation.

The quantity of data collected in this study varied between survey types primarily due to the way in which the data was collected. Species identified from the SSS were all recorded in the laboratory once a month for six months, whilst the MMS utilised field-based identification only once following the deployment period of eight months. This is believed to be the primary reason for the significant difference between the number of species identified within the SSS and MMS, as there were six times as many samples collected and laboratory based analysis allows for the identification of more cryptic and smaller organisms that may be missed during field identification. A consideration for future applications would be to adapt the MMS survey procedure to include monthly field examinations, thus incorporating the element of succession and seasonality, which would likely increase the total number of recorded species and reduce the disparity between the MMS and SSS. Having three of each survey type deployed within the port provided minimal replication, particularly when considering the level of replication required for powerful statistical analyses. This is also an important factor when considering the probability of detection of rare species. 
Many studies that use settlement panels to detect NNS do not make reference to the probability of detection when determining appropriate sample sizes (e.g. CanningClode et al., 2013; Bangor University, 2015; Hurst, 2016; Marraffini et al., 2017), however it is an important factor in determining the confidence that all NNS would be identified if present within a system and therefore the reliability of the survey method (Floerl et al., 2012; Ma, 2020). Within ports it would be difficult to significantly increase the sample as the availability of sites to deploy materials that satisfy both the research aims and port authorities are generally very limited (HELCOM, 2013). Due to the practicalities of working within active ports there must be leeway for a degree of compromise between increasing the number of replicates and operating safely within a potentially dangerous environment. I suggest therefore that sample size should be increased, with a view to increasing the probability of detecting NNS, when it is possible to achieve this safely. It follows that the importance of this research tends more towards the descriptive aspect of identifying species and the applied focus of informing future port monitoring and biosecurity management strategies, in line with current legislation (Environment (Wales) Act, 2016; Regulation (EU) No 1143/2014). Whilst both surveys yielded quantified measures of species richness and abundance, these were more easily calculated from the SSS due to the standardised size of PMMA tiles compared to the varied surface areas of materials used within the MMS. A consideration for port authorities applying these methods in the future would be to reflect on what sort of data would be valuable. If it is simply a case of listing which organisms are inhabiting the port, then quantified data would be less of a priority.

There are several practical aspects that need to be considered, which weigh for and against each survey type: cost, time, level of expertise required, and equipment required. For each of these factors the differences between each survey types comes down to the use of laboratory-based sample analysis. Each survey type cannot be separated in terms of the time required to deploy and retrieve materials; however, the more in-depth laboratory analysis takes considerably longer than fieldbased analysis. Laboratory analysis also requires a higher level of expertise and equipment than would be required to do more basic visual analysis in the field. 
These factors contribute to the overall cost that would be incurred for port authorities to implement the survey methods, with the accessibility of experienced taxonomists along with the necessary equipment and resources being an important consideration for future applications.

It should also be noted that settlement surveys are just one way in which to monitor NNS and describe the wider fouling community. The use of molecular techniques such as metabarcoding and the isolation of environmental DNA (eDNA) from water samples is becoming increasingly popular in the field of invasion biology, where the applications can include screening for target NNS and tracing the origin of NNS as well as more broadly identifying organisms present in a specific environment (Rius et al., 2015). Collecting water samples for the extraction of eDNA can be successfully and safely conducted within port environments, and has been proven effective at identifying some of the NNS identified within this study, suggesting this a viable option for NNS screening (Borrell et al., 2017; Holman et al., 2019). The use of eDNA and metabarcoding for the quantification of abundances, as well as being able to identify all organisms to species level, is perhaps currently limiting the use of this method. Alternatively, underwater video and the use of remotely operated vehicles (ROVs) can be an effective tool in identifying visually distinctive species and can cover large areas habitat (e.g. Cánovas-Molina et al., 2016, Meyer et al., 2020). In the past, the use of ROVs has generally been constrained by cost to broad scale and meso-scale surveys (Bo et al., 2014; CánovasMolina et al., 2016), however there is now an increasing use of mini-ROVs for smaller scale surveying which would be possible within ports (Buscher et al., 2020). Whilst video methods are likely to miss detail when it comes to identifying cryptic species, identifying organisms to species level, and providing a detailed insight into community structure, the speed with which data can be collected means this method could prove effective in screening for visually distinctive NNS within ports. On balance, data obtained from the settlement surveys outlined here are currently the most effective compromise between cost, effort and level of detail, that can provide useful insights for managers into community structure and community development, which may be overlooked by alternative survey methods. 


\subsection{Conclusion}

Deployment of the two survey designs outlined in this study was successful in terms of both overcoming the major constraints of conducting field surveys within active ports as well as providing a comprehensive description of the fouling communities present within the Port of Swansea. This success hinged on developing a strong working relationship with Associated British Ports, the operator of the Port of Swansea, which enabled for effective planning and implementation of surveys; a point which would likely be essential for conducting further surveys within active ports. A total of 38 species were recorded to species level, including 7 non-native species (NNS). Communities were found to be dominated by filter feeders, with large abundances of the solitary ascidians Ciona intestinalis and Ascidiella scabra as well as the non-native bryozoan Bugulina stolonifera. It is thought that some of these filter feeders, particularly A. scabra, serve as keystone species providing settlement surfaces and thereby supporting further colonisation by additional taxa. Community succession was evident over the course of the survey period which I suggest here may be driven by the succession of $A$. scabra by colonial ascidians. Colonial ascidians increase in colonisation as the abundance of A. scabra falls, leading to a change in habitat type and therefore community structure. Material type was found to play a significant role in determining community composition, and the knowledge gained on which materials support greater species richness or higher proportions of NNS has considerable implications for port management and could prove essential in developing biosecurity and biodiversity plans. There is little concern over the NNS recorded from within the port, given that the only NNS not to have been previously recorded in South Wales are unlikely to survive in the environmental conditions of the highly tidal Bristol Channel. Transport of NNS from port-to-port through ballast water or hull fouling is the only real concern regarding further dispersal of NNS, highlighting the importance of the port to continue adhering to biosecurity guidelines. 
A combination of both survey types is the clear approach in terms of providing a detailed analysis of fouling communities as well as offering practical insights to stakeholders regarding port management. It is recommended that the practical applications of survey implementation, particularly in terms of the available expertise and resources, prior to the deployment of survey materials, need to be carefully considered. 


\section{CHAPTER 2: Fouling communities and non-native species within five ports along the Bristol Channel, South Wales, UK}

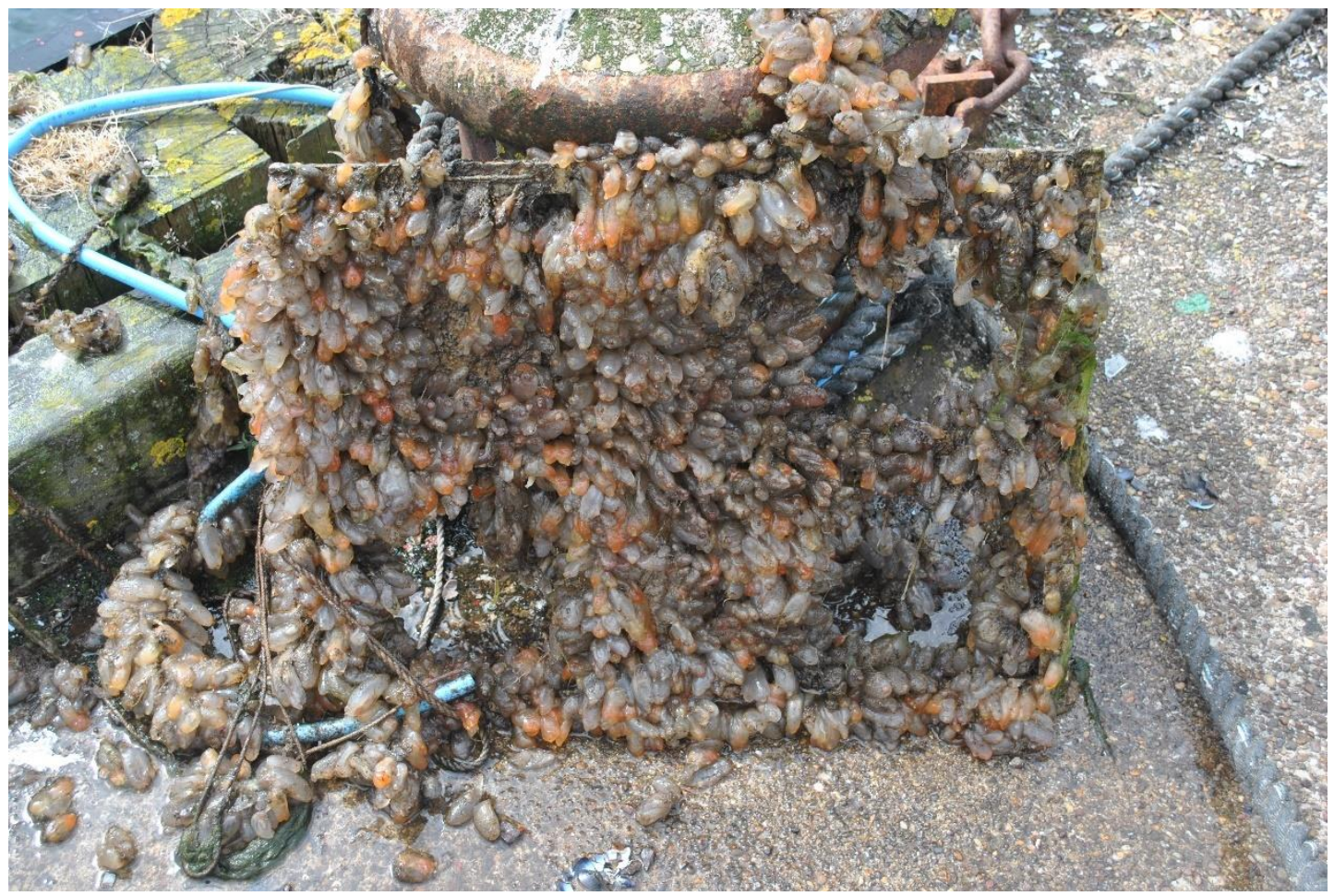

* A version of this chapter has been published as: Holmes, $\mathbf{S}$ and Callaway, R., 2021. Fouling communities and non-native species within five ports along the Bristol Channel, South Wales, UK. Estuarine, Coastal and Shelf Science. 252, 107295. 


\subsection{Abstract}

Non-native species (NNS) are widely regarded to be one of the major threats to the loss of biodiversity worldwide. Maritime trade is the primary pathway for the transport and introduction of aquatic NNS around the world, and ports are central to this network. Our knowledge of port communities and the NNS they contain is limited, with ports often remaining unsurveyed for decades, which was the case within the studied region. Settlement plates were deployed for 10-11 months at five commercial ports along the Bristol Channel and Severn Estuary in South Wales, UK. I report unique communities in each of the ports with salinity being the main driver for differences among locations. Eleven NNS were identified across all ports with non-native to native species proportions ranging from 0.13 to 0.33 in each port. Most of these NNS are known to exist in the region and are 'established' species within the UK. High variation in community structure and NNS composition among all ports independent of geographic proximity highlights the importance of monitoring individual ports with a view to implementing bespoke, effective NNS management strategies.

\subsection{Introduction}

Aquatic non-native species (NNS) have long been associated with the loss of biodiversity and their direct impacts on native species, habitats and ecosystems (Bax et al., 2003; Molnar et al., 2008; McGeoch et al., 2010; Blackburn et al., 2014). Whilst documenting the impacts of NNS is vital in developing an understanding of how damaging the spread of species can be, as well as raising the profile of the problem of NNS, the focus must remain on managing species introductions in order to minimise impacts. Management of NNS is most successful following the early detection of a species introduction to a new location, with management options becoming limited as species become established (Blackburn et al., 2011; Giakoumi et al., 2019). Monitoring or screening for early NNS introductions is therefore an essential aspect of management (Pyšek and Richardson, 2010; Blackburn et al., 2011).

Maritime trade is widely considered to be the primary pathway for the spread of marine NNS worldwide (Ruiz et al., 1997; Katsanevakis et al., 2013; Seebens et al., 2013; Williams et al., 2013; Bailey, 2015). 
At the centre of all maritime trade are ports, making these areas particularly high risk for the introduction of NNS (Bailey, 2015). A wider concern surrounding ports is the potential role that these areas may play in the invasion of species to a region by serving as vectors for NNS into adjacent natural systems, following an initial introduction into the port (Floerl et al., 2009). Ports are often isolated locations, where water conditions can be influenced by a range of factors including freshwater input and industrial port processes. Ports connected to the same water body may not therefore share the same, or similar, environmental conditions or communities, including NNS.

Ports are often a key focus in the monitoring of NNS, with a view to identifying novel species introductions early and allowing the most effective system of management to be implemented (Cohen et al., 2005; Marins et al., 2010; Borrell et al., 2017; Travizi et al., 2019). Port fouling communities worldwide are often dominated by sessile benthic organisms, included a large number of ascidians, associated with the hull fouling transport vector (e.g. Cohen et al., 2005; Marins et al., 2010; Giachetti et al., 2019). However, lack of data from within UK ports and the evident variation between port communities elsewhere in the world highlights the importance of monitoring port fouling communities in unstudied regions.

Survey methodologies widely used to detect marine NNS in ports, marinas and associated habitats, generally take the form of a settlement study (e.g. Arenas et al., 2006; Floerl et al., 2012; Mineur et al., 2012; Marraffini et al., 2017). These surveys have been used extensively around the world in various habitats and are regarded to be effective in the detection and identification of NNS (Floerl et al., 2012; Marraffini et al., 2017). Ports can be hostile places to carry out research, with safety being a key factor when working amongst large machinery, vessels, and associated cargo. Safe locations from which to deploy materials or access existing materials, necessary for conventional survey methods, can be rare within ports. Many settlement studies also overlook temporal variation in communities, despite succession being a key feature of fouling communities (Berntsson and Jonsson, 2003; Langhamer et al., 2009; South et al., 2019). With a view to detecting all NNS that may be present within a fouling community, the application of a survey which takes temporal variation into account may prove effective. 
Large scale strategic monitoring of NNS in Great Britain aims, in part, to develop 'early detection, surveillance, monitoring and rapid response' (UK Government, 2015). Efforts have so far been made to increase the involvement of various organisations and incorporating citizen science with the goal of increasing the amount of data being recorded in the public domain (UK Government, 2015). Ports, however, being privately owned and privately run industrial areas offer several complications for this strategic monitoring effort. For safety and access reasons large scale citizen science cannot be applied within ports, and certain survey types may not always be suitable for use. It has been noted that the spatial resolution for NNS monitoring is low across the whole of Europe, with many habitats not being consistently and regularly monitored (Painting et al., 2020). Whilst legislation such as the Water Framework Directive (Council Directive 2000/60/EC), the Marine Strategy Framework Directive (Council Directive 2008/56/EC) and the EU Biodiversity Strategy (COM (2011) 0244, final) drive the notion of preserving natural biodiversity and the monitoring and management of NNS, there is no clear or standardised protocol to assist port operators in achieving this (Boon et al., 2020). Developing a standardised survey protocol may be a step towards improving NNS monitoring within ports, increasing the data flow into large scale management strategies and improving our ability to minimise the risk that NNS pose to our native species and habitats, as well as coastal industry.

The aim of this study was to understand factors influencing the fouling communities in active ports, and specifically fouling of NNS. The objectives of this study were to:

1. Describe the fouling communities and detect NNS present in five ports in South Wales, UK.

2. Quantify aspects of community succession (community composition, abundance, coverage) over the course of an 11-month deployment of settlement plates.

3. Assess the influence abiotic variables have on determining fouling community composition and the proportion of NNS within port communities. 


\subsection{Materials and methods}

\subsubsection{Study area}

Research was conducted within the ports of Newport, Cardiff, Barry, Port Talbot and Swansea, each located along a 55-mile section of the Bristol Channel and Severn Estuary in South Wales, UK (Figure 15). These ports are all commercial ports owned and operated by Associated British Ports (ABP) and are each connected directly to the Bristol Channel by a lock. There is only one published survey from the 1950s of Swansea (Naylor, 1957) and none from the remaining four ports. Water levels are maintained in all shipping areas to at least $10 \mathrm{~m}$ depth whilst the Bristol Channel itself experiences a tidal range of up to $13 \mathrm{~m}$. These ports may therefore support unique subtidal communities that are influenced by a range of factors including environmental conditions as well as the potential introduction of species through maritime trade.

Table 2. Salinity, shipping traffic and species richness of ports at the Bristol Channel (East to West). Salinity range over 11 months (2019), mean shipping traffic calculated from ABP logs; total number of species colonising settlement plates in 2019.

\begin{tabular}{lccc}
\hline Port & Salinity & $\begin{array}{c}\text { Shipping traffic } \\
\text { (mean month }^{-1} \text { ) }\end{array}$ & $\begin{array}{l}\text { Total number of } \\
\text { species }\end{array}$ \\
\hline Swansea & $28-30$ & 81 & 30 \\
Port Talbot & $0.1-0.3$ & 8 & 6 \\
Barry & $22-24$ & 22 & 25 \\
Cardiff & $1-12$ & 42 & 14 \\
Newport & $8-16$ & 73 & 13 \\
\hline
\end{tabular}

Salinity levels, measured using an Aquaread AP-5000 probe, varied between ports from freshwater in Port Talbot to near marine salinity in Swansea (Table 2). Barry, Cardiff and Newport all occupy a range of brackish salinities, with Cardiff the only port displaying any stratification whereby salinity can increase from 1 in surface water to 12 at a depth of $7 \mathrm{~m}$. The variation in salinities between ports is due to the way in which water levels are maintained. Port Talbot, Cardiff and Newport all have a direct freshwater inflow from nearby rivers, whilst Barry and Swansea ports rely on the pumping of water from the Bristol Channel at high tide. 
Water in the Port of Port Talbot is consistently at least $3{ }^{\circ} \mathrm{C}$ warmer than the other ports due to the use of dock water for cooling within an adjacent steel works. The effluent of this process is returned into the dock which results in its warming (ABP, personal communication).

ABP provided quantitative data of the number of ships passing through port locks. The data was summarised from 2018-2019, and average traffic per month was calculated (Table 2). Fewer ships enter Port Talbot docks compared to the other four ports as most of the trade in Port Talbot takes place outside of the docks in a tidal harbour.

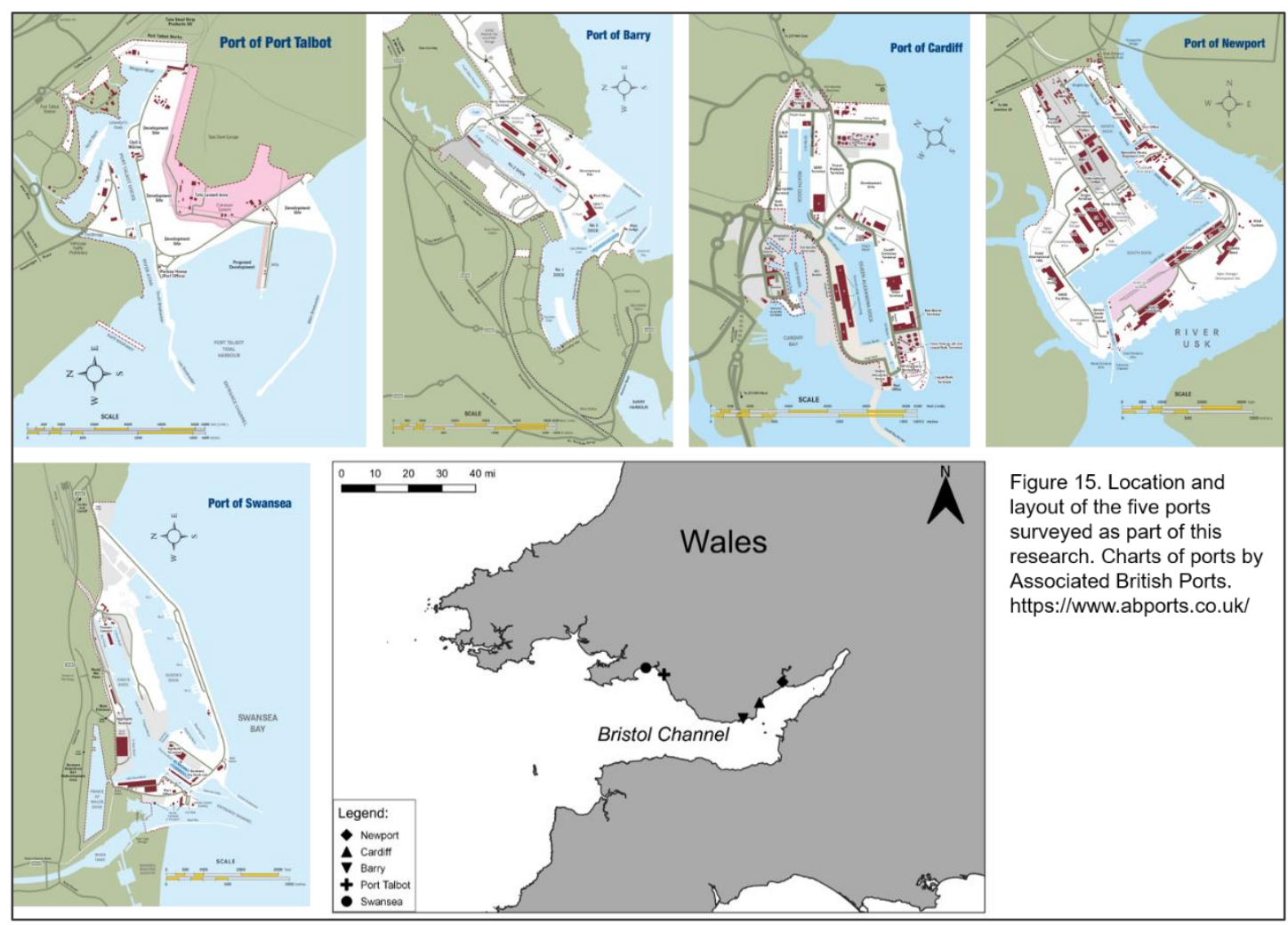

\subsubsection{Survey design and data collection}

The study was designed to record succession of ecological parameters (community composition, species richness, abundance, percentage cover) over six sampling dates during a 10 - 11-month deployment period in 2019. This also maximised the opportunity to detect as many species as possible over almost one year. Three sites in each port were chosen for the deployment of settlement plates and each of site was regarded as an independent replicate for the port location. 
Poly(methyl methacrylate) (PMMA) tiles $(15 \mathrm{~cm} \times 15 \mathrm{~cm}$, grey in colour) were used as the settlement material. 6 tiles per site were lightly sanded using 40 grit sandpaper and mounted, using cable ties, vertically within an aluminium frame (Figure 16). Each frame was suspended in the water column using polypropylene rope, attached to a fixed surface mounting point (e.g. a mooring bollard; Figure 16). Frames were suspended initially to a depth of approximately $4 \mathrm{~m}$, although the water level in the port can vary meaning that depth did not remain constant during deployment. Frames were deployed in December 2018 and retrieved in October/ November 2019. One PMMA tile was removed from each frame every 6 - 8 weeks and returned to the laboratory for analysis, where macrofauna were visually identified to the lowest possible taxon from both the front and the reverse side of the tile using guides such as the Handbook of the Marine Fauna of North-West Europe (Hayward and Ryland, 2017), British Marine Amphipoda (Lincoln, 1979) and Linnean Society taxonomic resources. Tiles were photographed in the laboratory from which percentage cover was calculated using ImageJ software.

Temperature was measured continuously using Tinytag sensors, recording every 30 minutes, which were attached directly to the aluminium frame holding the settlement plates. Measures of salinity, temperature, depth, $\mathrm{pH}$, dissolved oxygen (DO; $\mathrm{mg} / \mathrm{L}$ and \% saturation), and oxidation reduction potential (ORP) were taken approximately every 4 weeks throughout the survey period using an Aquaread AP5000 probe (data displayed in Appendix II).

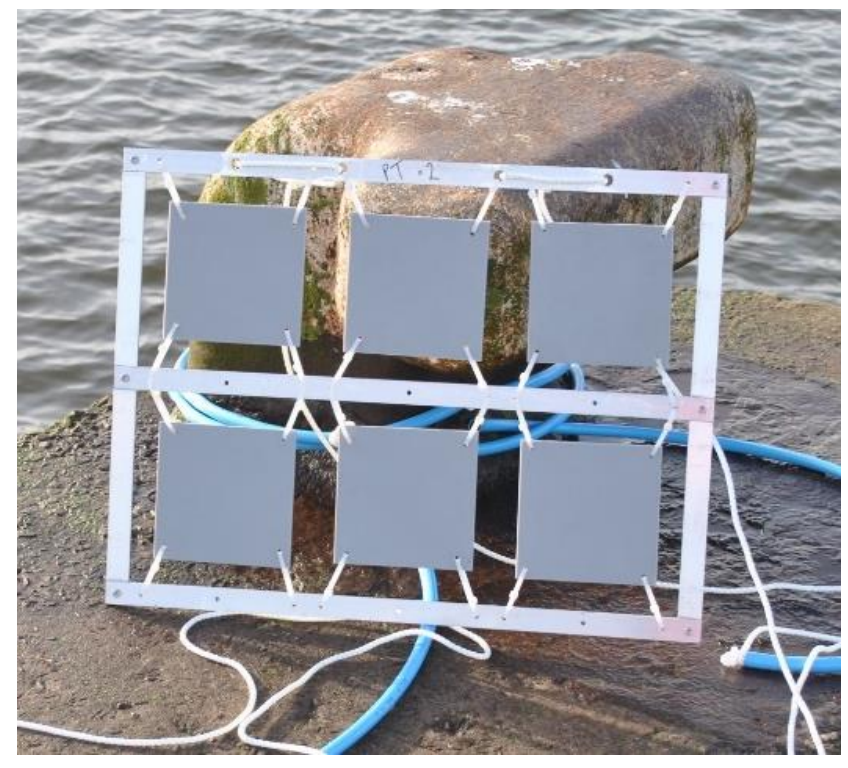

Figure 16. Survey materials prior to deployment. 


\subsubsection{Data analysis}

Whole community analysis, comparing samples collected within each port at each sampling occasion, was completed using Primer 6 v.6.1.13 with PERMANOVA v.1.0.3 (Anderson et al., 2008). Separate analyses outlined below were conducted using square root transformed abundance counts of countable species and the presence/ absence of all recorded species to incorporate both countable and noncountable species.

All samples without colonisation were removed from multivariate analysis (January and some of March 2019). A similarity matrix using a Bray Curtis similarity index was created for all samples with colonising species. A non-metric multidimensional scaling (nMDS) plot and PERMANOVA were completed from this matrix. A PERMANOVA test was designed with the fixed factors 'Port' and 'Month' and values in the similarity matrix as response variable to analyse the effect of the different ports and months on the fouling communities. The model used the permutation of residuals under a reduced model with type III (partial) sum of squares and 9999 permutations. Pairwise PERMANOVA analyses among 'Port' groups and 'Month' groups were also completed using this same model design. This design was also used to analyse the effect of Port and Month as factors both on the number of non-native species and the frequency of occurrence of NNS within samples based on a similarity matrix generated using Euclidian distances. Inclusion of the factor 'Month' here partially addresses aim two, meaning to better understand the temporal succession of colonisation.

Due to a high variation in community composition between ports, the factor 'Month' was also analysed independently for each port, further addressing aim two. Kruskal-Wallis tests were conducted to determine the effect of month on three measures of colonisation: number of species, total abundance (countable species), and percentage cover (all species) within each port. Pairwise Dunn's tests were used to identify significant differences in the colonisation between months. These analyses were carried out within R v.3.6.2 (R Core Team, 2017). 
A distance-based linear model (DistLM) with distance-based redundancy analysis plot (dbRDA) was used to quantify how much variation between samples could be attributed to certain environmental and human driven variables, addressing the third aim of this study to understand the importance of environmental factors in the colonisation process. Variables used were temperature, salinity, $\mathrm{pH}, \mathrm{DO}(\%$ saturation), ORP, and ship traffic. No autocorrelation between variables was detected based on correlations generated using a draftsman plot in Primer 6. The DistLM was based on values in a Bray Curtis similarity matrix based on square root transformed abundance data. The DistLM used a stepwise selection procedure and R2 selection criterion with 9999 permutations. When analysing the influence of these variables on the number of non-native species and frequency of occurrence of NNS within samples the DistLM was based on a similarity matrix created using Euclidian distances.

\subsection{Results}

\subsubsection{Fouling communities}

Swansea had the highest recorded number of species, followed by Barry, Cardiff, Newport, with Port Talbot having the fewest (Table 2). Fouling communities were numerically dominated by different species in each port (full list of recorded taxa in Supplementary Table S3). Mytilus edulis and Ciona intestinalis were the only species to feature as the most abundant species in more than one port. Across all five ports, Amphibalanus improvisus was the most abundant species, with an average of over 800 individuals recorded per $450 \mathrm{~cm}^{2}$ sample (front and reverse side of a $15 \times 15 \mathrm{~cm}$ tile) from the Port of Newport. Sampling photos available in Appendix III.

The similarity in fouling communities among ports and months was visualised using an nMDS (Figure 17). Both 'Port' and 'Month' as fixed factors were found to significantly influence the observed fouling communities based on presence/ absence data (PERMANOVA: Port, pseudo-F = 16.372, $\mathrm{p}=0.0001$; Month, pseudo- $\mathrm{F}=5.024, \mathrm{p}=0.0001$ ), as well as the abundance of countable organisms (PERMANOVA: Port, pseudo-F = 9.995, $\mathrm{p}=0.0001$; Month, pseudo-F $=$ 4.436, $\mathrm{p}=0.0001)$. 
Communities observed within each port were found to be significantly different to all other ports in a post-hoc pairwise analysis, based both on the presence/ absence of all observed species and the abundance of countable organisms (PERMANOVAs, $\mathrm{p}<$ 0.05). Communities observed between all month pairs were significantly different to one another (PERMANOVAs, $\mathrm{p}<0.05$ ), except for March and May, September and October (both for presence/ absence and abundances) and July and September (abundances only).

A SIMPER analysis was completed to identify which species are driving the dissimilarity in communities observed between ports. The dissimilarity between ports with large differences in salinity and temperature, e.g. Swansea and Port Talbot, was driven by species which were present in one port but absent in the other. Swansea and Port Talbot had an average dissimilarity of $100 \%$ of which ca. $45 \%$ was contributed by three species: Balanus crenatus, Ciona intestinalis and Ascidiella scabra. These species were present in high abundance in the Port of Swansea (mean abundance \pm SE: B. crenatus, $10.6 \pm 2.6450 \mathrm{~cm}^{-2}$; C. intestinalis, $57.7 \pm 22.8450 \mathrm{~cm}^{-}$ 2; A. scabra, $85.9 \pm 29.1450 \mathrm{~cm}^{-2}$ ) and absent from the port of Port Talbot. Ports with similar salinity had considerable overlap in the community composition and the dissimilarity between them was driven by the relative abundance of species present in both ports. Cardiff and Newport, for example, had an average dissimilarity of ca. $75 \%$ of which over $55 \%$ was attributed to three species present in both ports with different abundances: Amphibalanus improvisus (mean abundance \pm SE: Cardiff, $65.7 \pm 26450 \mathrm{~cm}^{-2}$; Newport, $654.6 \pm 326.7450 \mathrm{~cm}^{-2}$ ), Ficopomatus enigmaticus (mean abundance \pm SE: Cardiff, $197.4 \pm 91.7450 \mathrm{~cm}^{-2}$; Newport, $0.4 \pm 0.4450 \mathrm{~cm}^{-2}$ ), Gammarus zaddachi (mean abundance \pm SE: Cardiff, $0.1 \pm 0.1450 \mathrm{~cm}^{-2}$; Newport, $\left.3.2 \pm 2.5450 \mathrm{~cm}^{-2}\right)$. 


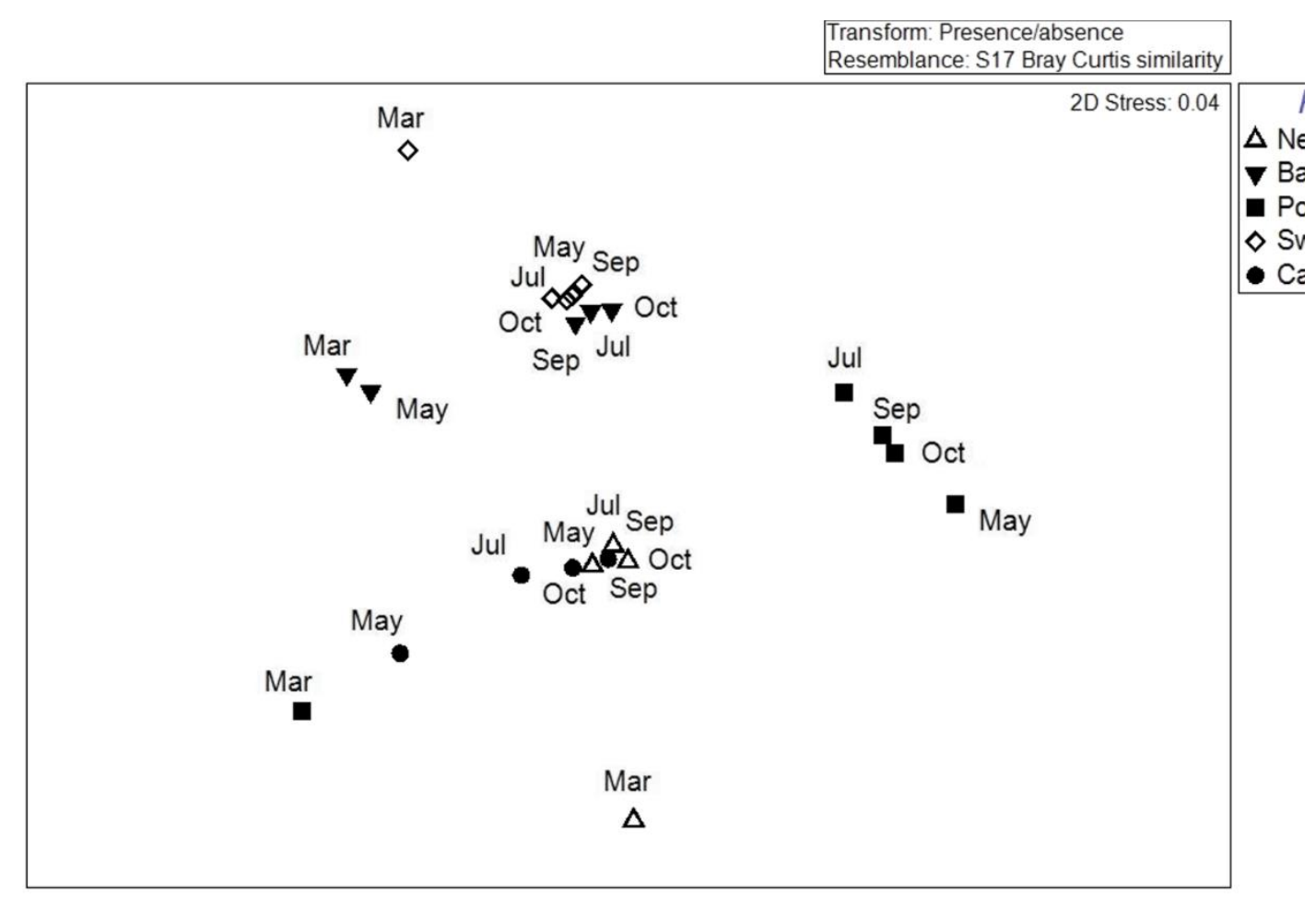

Figure 17. nMDS plot of fouling community samples collected within 5 ports at bimonthly intervals throughout 2019. Raw data averaged for each sample point ( $\mathrm{n}=$ 2-3) and transformed using a presence/ absence transformation. Plot based on a resemblance matrix created using a Bray Curtis similarity index. Samples without any species were removed.

Due to the high variation in community structure among ports the influence of the factor 'month' on fouling communities was also analysed independently for each port. Whole community analysis within ports revealed 'month' to be a significant factor influencing community composition in the ports of Newport, Cardiff, Barry and Swansea (PERMANOVAs: Newport, pseudo-F = 3.9053, p = 0.0015; Cardiff, pseudo- $\mathrm{F}=8.7874, \mathrm{p}=0.0001$; Barry, pseudo- $\mathrm{F}=3.1573, \mathrm{p}=$ 0.0001 ; Swansea, pseudo-F $=2.802, \mathrm{p}=0.0013$ ). Three univariate measures of colonisation were analysed (species richness, total abundance of countable species and percentage cover of all species; Figure 18) to determine the effect of succession over time ('month') on the observed communities within each port. 'Month' was a significant factor determining the observed number of species, abundance, and percentage cover in the ports of Newport, Cardiff, Barry and Swansea (KruskalWallis test, $\mathrm{p}<0.05)$. 
Only percentage cover was found to be significantly influenced by the factor 'month' within the port of Port Talbot (Kruskal-Wallis test, $\mathrm{p}<0.05$ ). Pairwise tests revealed that the effect of month was largely influenced by differences in colonisation between early months (January and March) and later months (July, September, October; Figure 18).

The influence of several abiotic variables (salinity, temperature, $\mathrm{pH}, \mathrm{DO} \%$ saturation, ORP, ship traffic) on the observed communities was analysed using a Distance-based Linear Model (DistLM) and visualised using a dbRDA plot (Figure 19). Salinity and dissolved oxygen concentration (DO) together were found to explain $23 \%$ of the variation observed between samples (DistLM; variable: salinity, pseudo-F $=7.733, \mathrm{p}=0.0001$, prop $=0.132$; variable: $\mathrm{DO}$, pseudo-F $=6.689, \mathrm{p}=$ 0.0001 , prop $=0.102)$. Temperature was found to explain an additional $8 \%$ in variation (DistLM, pseudo-F $=5.543, \mathrm{p}=0.001$, prop $=0.078$ ) whilst the remaining three variables included in the analysis (ship traffic, ORP, $\mathrm{pH}$ ) contributed only $9 \%$ combined. Ship traffic, ORP and $\mathrm{pH}$ were though all significant factors in this analysis (DistLM, $\mathrm{p}<0.05$ ) despite accounting for a small percentage of the total variation. In total these six variables combined explained $40 \%$ of the variation observed among samples. 

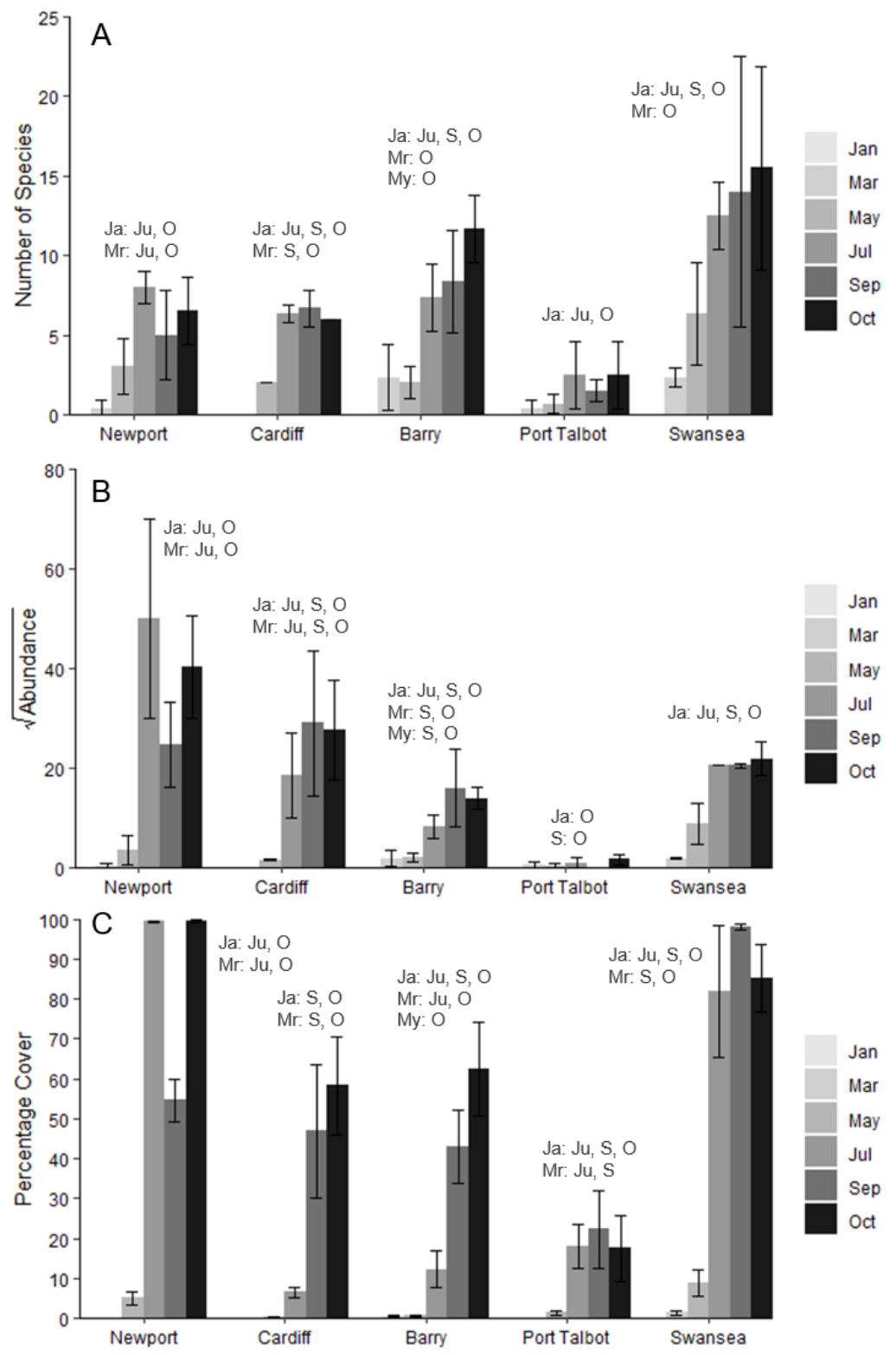

Figure 18. Colonisation of settlement plates at five ports over 10 months. Records per sampling month in the ports of Newport, Cardiff, Barry, Port Talbot and Swansea. A: Mean species richness $450 \mathrm{~cm}-2$. B: mean square root abundance per sample. C: Mean percentage cover per sample. Error bars show standard deviation. $\mathrm{n}=3$ with the exception of Newport (Sep, Oct), Cardiff (Oct), Port Talbot and Swansea (Jul, Sep, Oct), where $n=2$. Abundance based on countable organisms only, percentage cover based on all organisms. Results of significant pairwise Dunn's tests $(\mathrm{p}<\alpha / 2$, where $\alpha=0.05)$ between month groups within each port are indicated by letters above each set of bars. 'Ja: Ju, O' for example indicates a significant difference between the month pairs January and July and January and October. 


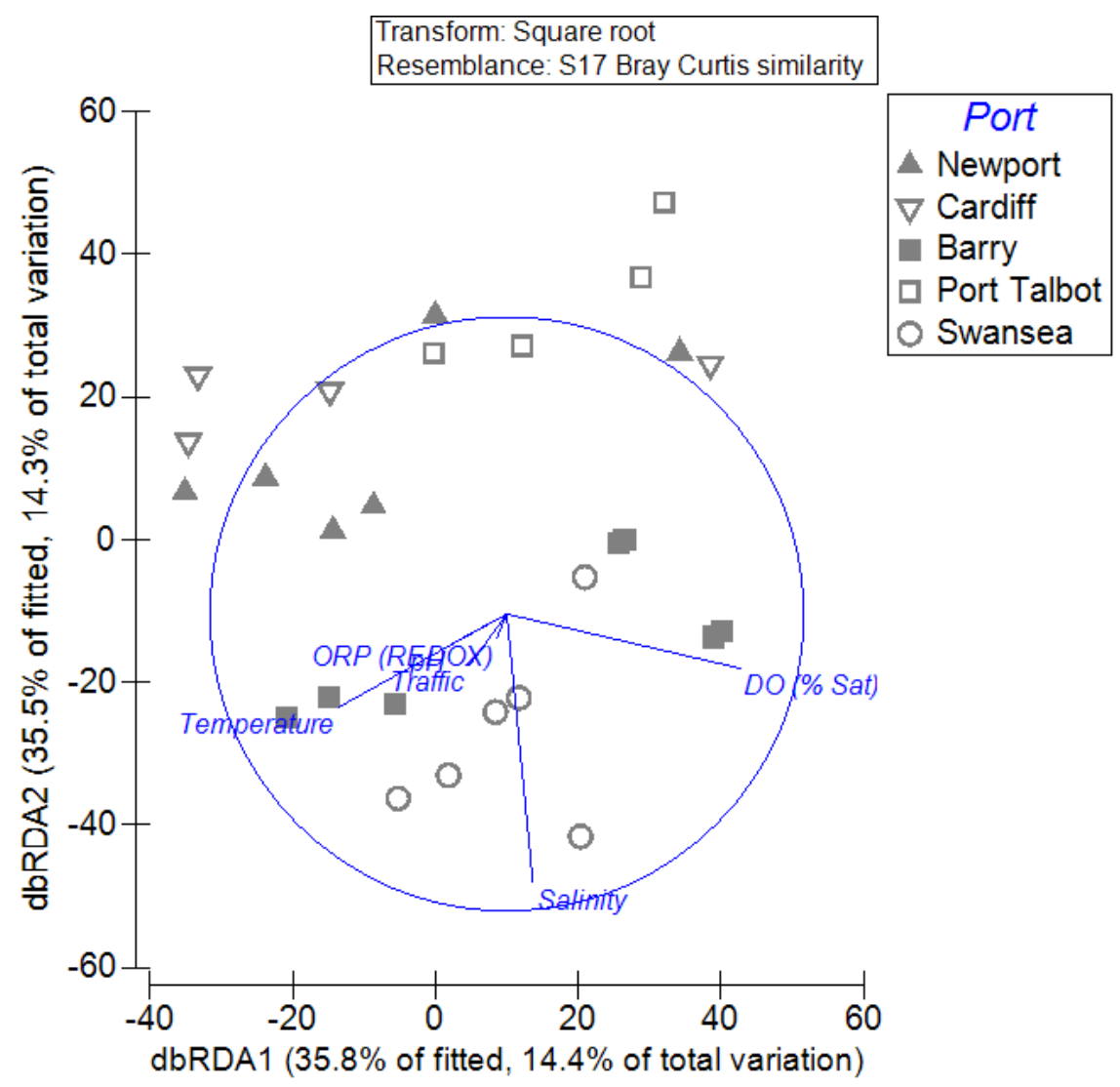

Figure 19. Distance-based redundancy analysis (dbRDA) exploring the amount of variation between communities that may be explained by six abiotic variables (salinity, temperature, $\mathrm{pH}, \mathrm{DO}, \mathrm{ORP}$, ship traffic). Analysis completed using a similarity matrix based on a Bray-Curtis similarity index and square root transformed abundance data for countable species.

\subsubsection{Non-native species (NNS)}

11 NNS were identified across the five ports surveyed within this study (Table 3). The Port of Barry had the highest recorded number of NNS with seven species, whilst Port Talbot had only two. All but one species, Brachynotus sexdentatus, are considered to be established species within Great Britain and all but one species (Cordylophora caspia) have been previously recorded from within the Bristol Channel area (NBN Atlas, 2020). Port Talbot had the highest proportion of NNS within communities, whereby one in every three species was non-native, Swansea had the lowest proportion of NNS. In descending order, the proportion of NNS was Port Talbot 0.33, Barry 0.24, Cardiff 0.21, Newport 0.15, Swansea 0.13. 
The colonisation of the three most dominant NNS based on total recorded abundance (Amphibalanus improvisus, Bugulina stolonifera, Ficopomatus enigmaticus) varied both spatially in terms of which ports they colonise, and temporally in terms of the speed of population development and how the population was sustained thereafter (Figure 20).

Both Port and Month were found to be significant factors in determining the number of NNS recorded in samples (PERMANOVA; factor: Port, pseudo-F = 2.842, $\mathrm{p}=0.0362$; factor: Month, pseudo-F $=20.213, \mathrm{p}=0.0001$ ). As environmental conditions and the amount of shipping vary between ports and months, a Distancebased Linear Model (DistLM) was used to determine whether certain environmental variables or shipping were driving the observed differences in the number of NNS between ports. Temperature and salinity combined accounted for over $30 \%$ of the observed variation between samples (DistLM; variable: temperature, pseudo-F = 25.095, $\mathrm{p}=0.0001$, prop $=0.241$; variable: salinity, pseudo- $\mathrm{F}=10.065, \mathrm{p}=0.0016$, prop $=0.087)$. All other variables included in the analysis (DO, ORP, $\mathrm{pH}$, ship traffic) accounted for a combined $<6 \%$ of variation in the number of NNS observed among samples (DistLM, $\mathrm{p}>0.05$, prop $<0.03$ ).

By contrast, Port and Month as factors were found to have no significant influence on the observed frequency of occurrence of NNS in samples (PERMANOVA; factor: Port, pseudo-F = 1.593, $\mathrm{p}=0.212$; factor: Month, pseudo-F $=0.792, \mathrm{p}=0.538)$. Ship traffic was the only significant driver of variation in the frequency of occurrence of NNS; it explained $9 \%$ of the total observed variation (DistLM, pseuso-F $=5.41, \mathrm{p}=0.0231$, prop $=0.09$ ). All other analysed variables accounted for a remaining 1.5\% (DistLM, p > 0.05, prop < 0.006). Combined with ship traffic all factors explained $10.6 \%$ of the variation. 
Table 3. Non-native species recorded in South Wales ports. GB establishment status and existing presence in the Bristol Channel based on data from NBN Atlas (NBN Atlas, 2020). * indicates species previously known to inhabit the recorded port(s) but not the Bristol Channel.

\begin{tabular}{lllll}
\hline & & \# No. samples & & \\
where species & GB establishment & Existing Presence in \\
Taxa & Port(s) & 13 Established & Yes \\
Amphibalanus improvisus & Newport, Cardiff & 8 Established & Yes \\
Austrominius modestus & Barry, Swansea & 1 Unknown & No * \\
Brachynotus sexdentatus & Swansea & 8 Established & Yes \\
Bugulina stolonifera & Barry, Swansea & 11 Established & No * \\
Caprella mutica & Barry, Swansea & 5 Established & No \\
Cordylophora caspia & Cardiff, Port Talbot & 1 Established & Yes \\
Diadumene lineata & Barry & 6 Established & Yes \\
Ficopomatus enigmaticus & Newport, Cardiff & 3 Established & Yes \\
Monocorophium sextonae & Barry & 2 Established & Yes (in freshwater) \\
Mytilopsis leucophaeata & Port Talbot & 1 Established & Yes \\
Styela clava & Barry & & & \\
\hline
\end{tabular}



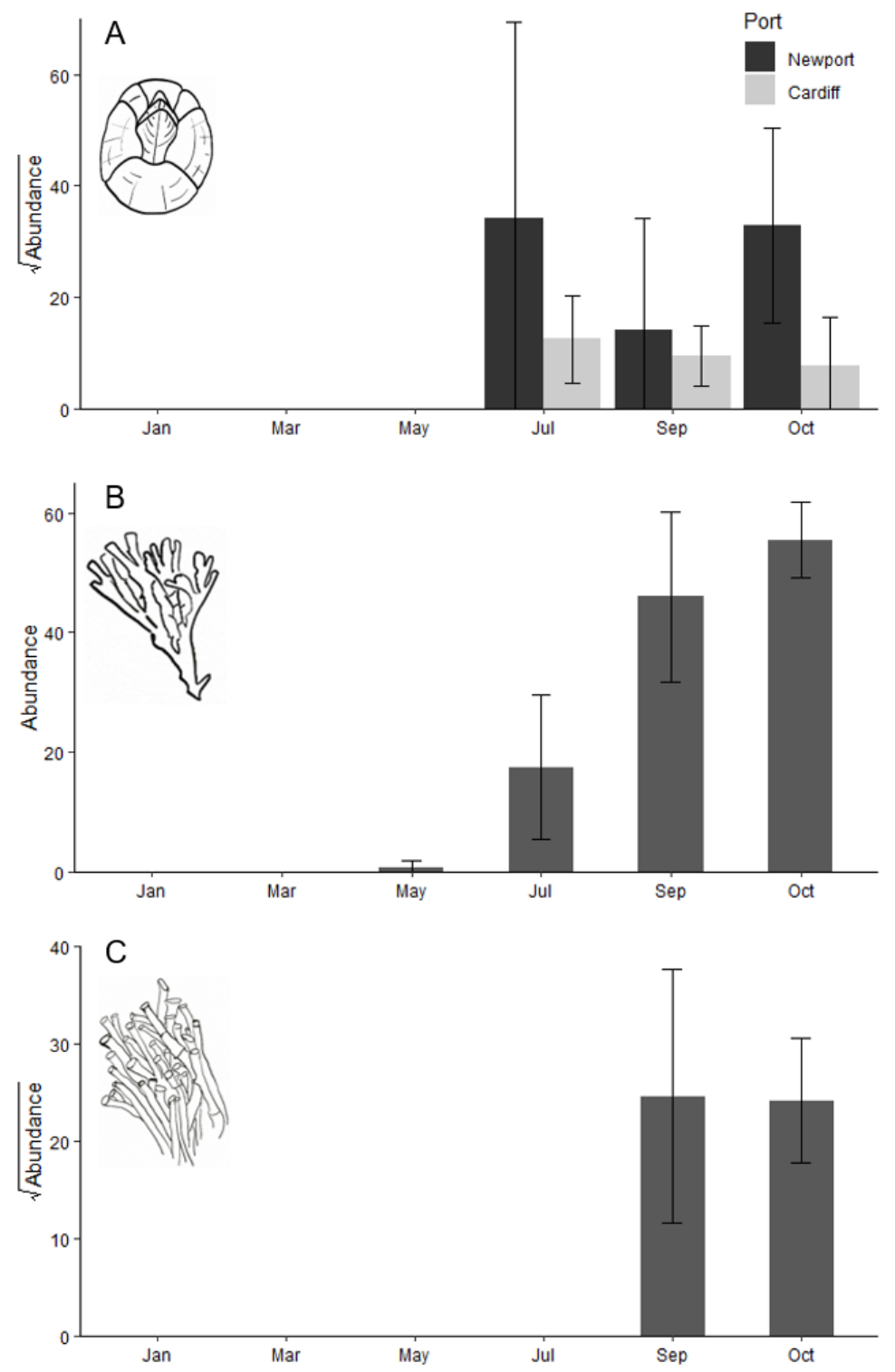

Figure 20. Spatial and temporal distribution of the three most abundant non-native species. A: Amphibalanus improvisus as recorded in the ports of Newport and Cardiff. B: Bugulina stolonifera as recorded in the port of Swansea. C:

Ficopomatus enigmaticus as recorded in the port of Cardiff. B. stolonifera and F. enigmaticus were each present in only one sample from within the ports of Barry and Newport, respectively, and these data were omitted from the figure. 


\subsection{Discussion}

The formation of fouling communities is known to be a complex process, influenced by a range of abiotic and biotic factors (Sutherland and Karlson, 1977; Glasby, 1999; Glasby, 2000; Nydam and Stachowicz, 2007; Giachetti et al., 2019; Leclerc et al., 2019). In this study fouling communities varied significantly among five ports located along the same body of water, the Bristol Channel and Severn Estuary (UK). Succession has also long been recognised as a feature in the formation of fouling communities (Berntsson and Jonsson, 2003; Cifuentes et al., 2010; Lindeyer and Gittenberger, 2011). Despite this, most studies aiming to describe fouling communities and to identify non-native species (NNS) do not consider a temporal factor (e.g. Cohen et al., 2005; Arenas et al., 2006; Floerl et al., 2012; Mineur et al., 2012). I identified succession of different aspects of community structure in each of the five studied ports. However, the patterns of succession were not consistent in all ports, with some plates being colonised rapidly early in the spring and others later in the season as well as disparity in the degree of change in community composition among ports. Variation in the degree of succession between communities is not uncommon (Berntsson and Jonsson, 2003; Nydam and Stachowicz, 2007; Ronowicz et al., 2014), and the particularly isolated nature and high variation in environmental conditions between the ports studied here likely contributed to the variation we see in the formation of their respective fouling communities.

Overall, $40 \%$ of the variation in the observed fouling communities could be explained by six abiotic variables recorded and analysed within this study (salinity, temperature, dissolved oxygen, $\mathrm{pH}, \mathrm{ORP}$, and ship traffic).13\% of the variation was explained by salinity alone which is concurrent with previous studies highlighting salinity as a significant factor in shaping fouling communities (MacGinitie, 1939; Charles et al., 2018; Pinnell and Turner, 2020). It appears though that other abiotic or biotic factors which were not measured within this study influenced the observed succession. Reported factors include founder effects (Lindeyer and Gittenberger, 2011; Vieira et al., 2018), predation (Nydam and Stachowicz, 2007; Giachetti et al., 2019), and habitat type (González-Duarte et al., 2018; Leclerc et al., 2019). The latter can be considered negligible or absent from this study given that the studied ports are artificial sites with similar habitat types. 
Founder effects and predation, however, may play a significant role in the succession and formation of the observed fouling communities. The effects of predation varied between studies, with some reporting a reduced species diversity in the presence of macropredators, while other studies found no significant effect (Nydam and Stachowicz, 2007; Giachetti et al., 2019; Leclerc et al., 2019). Founder effects may have influenced the succession and composition of the fouling communities. Solitary ascidians, found in high abundance in the ports of Barry and Swansea, may have served as founder species in community development (Lindeyer and Gittenberger, 2011). Ascidiella scabra has indeed been linked with succession in fouling communities within the Port of Swansea in a previous study (Holmes and Callaway, 2020). Habitat complexity is known to influence the species diversity of a community (Sueiro et al., 2011; Mendez et al., 2015). Solitary ascidians can colonise rapidly, forming complex 3-dimensional habitats capable of supporting a wide range of other taxa (Svane and Gröndahl, 1988). By contrast, barnacles, such as Amphibalanus improvisus, recorded in high abundance in the Port of Newport, form dense mats with little habitat complexity in comparison to solitary ascidians.

It follows then that the observed lower total abundance and higher species diversity in the ports of Barry and Swansea were possibly facilitated by the abundant solitary ascidians present. However, these biotic variables are inherently linked to environmental conditions within each port, particularly salinity. For example, whilst A. improvisus would be able to thrive in the environmental conditions in the Port of Swansea (Dineen Jr. and Hines, 1992) and may have been spatially outcompeted by ascidians, A. scabra would likely not tolerate the lower salinity environment in the Port of Newport (Hiscock, 2006). Brackish environments also tend to support lower diversity in general (Cognetti and Maltagliati, 2000), which likely contributed to the lower observed diversity in the brackish ports of Newport and Cardiff. It seems plausible that whilst founder effects may be crucial in forming the observed communities within each port, salinity remains the underpinning variable in determining community composition and explaining the variation in communities between these ports. 
A major concern regarding ports is the role they may play in the spread of NNS by serving as vectors into a region (Floerl et al., 2009). Eight of the 11 NNS recorded across the five ports have an existing presence within the Bristol Channel and Severn Estuary, the immediately adjacent water body to each port (NBN Atlas, 2020). Known effects of propagule pressure in aquatic invasion biology (Lockwood et al., 2005; Colautti et al., 2006; Wilson et al., 2008; Bacon et al., 2014) indicate a likelihood that the studied ports contributed to the introduction and establishment of NNS in this region, although it is impossible to quantify the role they played in this process due to a lack of historical data. The focus here will be on the three NNS that have to date not been recorded outside of the ports within this region: Brachynotus sexdentatus, Caprella mutica and Cordylophora caspia. Based on the environmental tolerances of each of these species, B. sexdentatus and C. caspia are at a low risk of spreading out of the ports where temperatures can range from $<5^{\circ} \mathrm{C}$ to $23^{\circ} \mathrm{C}$ and salinities from 17 to $>30$ (Henderson et al., 2012). B. sexdentatus is native to the Mediterranean Sea and is thought to have become resident within the Port of Swansea whilst artificial warming was taking place within the port, which ceased in the 1970s (Arenas et al., 2006). It is likely that B. sexdentatus has become adapted to the cooler water temperatures over time, and therefore may be able to tolerate the temperature ranges within the Bristol Channel, however it remains to be seen whether this is the case since B. sexdentatus has not been recorded from outside of the ports to date. It may be that the salinity range within the Bristol Channel is not favourable for this species, or that the low abundance within the port is limiting the potential for dispersal. C. caspia is a freshwater/ brackish species of hydroid, capable of tolerating salinities below 16 (Folino-Rorem and Indelicato, 2005), therefore unlikely to survive in the considerably higher salinities of the Bristol Channel.

In contrast, $C$. mutica has the environmental tolerance to survive in these conditions (Ashton et al., 2007a). C. mutica has a strong link to human activity in the marine environment, and particularly the maritime trade industry, having regularly been recorded within ports and marinas worldwide and rarely within natural habitats (Willis et al., 2004; Ashton et al., 2007b). This suggests that C. mutica is not successful at dispersing beyond enclosed environments, perhaps due to the lack of a larval stage in Caprellid amphipods (Thiel, 1998; Cook et al., 2007). 
It may also be due to the relatively recent dispersal of this species around the world, and the spread of populations out of ports may accelerate over time. A lack of records from outside of ports and marinas should therefore not be used to downplay the risk of spread. The relatively low abundances recorded for C. mutica, at present, across the five surveyed ports (total abundance of 50) does though reduce the risk of wider dispersal as recruitment is most likely low within the ports.

When considering the abiotic variables which drive the number of NNS within ports, temperature and salinity alone accounted for over $40 \%$ of the observed variation between samples. In general, the number of NNS was greater in samples collected from environments with higher salinity and temperature. Most aquatic NNS tend to be marine species, owing to the necessity to survive interactions with marine bodies of water in most ship transits (Carlton, 1996; Seebens et al., 2013; Bailey, 2015). This may contribute to the increased number of NNS in ports of near marine salinity, as observed within this study. The amount of ship traffic was not found to be a significant factor in determining the number of NNS, yet was a significant factor in determining the frequency of NNS within a samples. We believe this to be driven by the high proportion of NNS found in Port Talbot, where one in every three species was non-native, coupled with the considerably lower ship traffic by comparison with the four other ports. This does nevertheless highlight the importance of monitoring a range of port types and not focussing on the ones with the busiest traffic.

Surveying within these challenging environments limited the number of settlement plates that could be deployed, and therefore the number of replicates obtained. It also led to loss of some replicates during deployment. It should be noted that the low number of replicates here may have played a role in the observed statistical variation both spatially among ports and temporally between month groups. The use of frames with six settlement tiles was intended to accommodate increased total replicates as well as allowing for a temporal factor in the study. Such a survey design does however introduce potential pseudo-replication when comparing between month groups in individual ports, since settlement tiles are housed in close proximity within a single frame, thus reducing their independence due to possible interaction between species of individual tiles. 
The limited number of safely accessible deployment sites limited survey design option, and we feel that on balance the importance of surveying for temporal variation justified the less than desirable survey design. Results should though be viewed with caution and, for example, standard variations should not be overinterpreted, due to the limitations in terms of replication and sampling design.

In terms of management of NNS in ports, the findings of this study suggest that short-term monitoring efforts and resources could be focussed on ports which are more likely to support a larger number of NNS based on the environmental conditions. However, with a view to documenting all NNS within a region and therefore developing the most effective management strategies, long-term monitoring must not focus on any one port. Whilst larger and more active ports may be more at risk to novel introductions based on the amount of ship traffic they experience (Seebens et al., 2013, 2016; Sardain et al., 2019), smaller and historically active ports are likely harbouring NNS and may therefore pose a similar risk to the neighbouring natural systems. Developing an understanding of the species present within these ports may prove decisive in understanding if, how, and over what timescale NNS spread from a port into a natural habitat.

\subsection{Conclusion}

The high degree of spatial variation in the observed communities between ports showed that it cannot be assumed that any two ports, even in close proximity, will be colonised by similar fouling communities. Whilst environmental conditions can provide an indication as to the potential diversity within a port community, the high level of complexity surrounding the formation of fouling communities limits our ability to make assumptions based on environmental conditions alone. This is of importance when considering the monitoring of non-native species (NNS) within ports or similar isolated environments. The high degree of variation in the NNS recorded among ports highlights the importance of monitoring individual ports, with the aim of documenting the introduction of NNS and minimising the risk of spread by better understanding the range of NNS present in the region. Increasing the spatial and temporal resolution of community data from within the port environment could prove essential in implementing the most effective NNS management strategies. 


\section{CHAPTER 3: Material selection by fouling}

\section{non-native species present in port}

\section{environments}

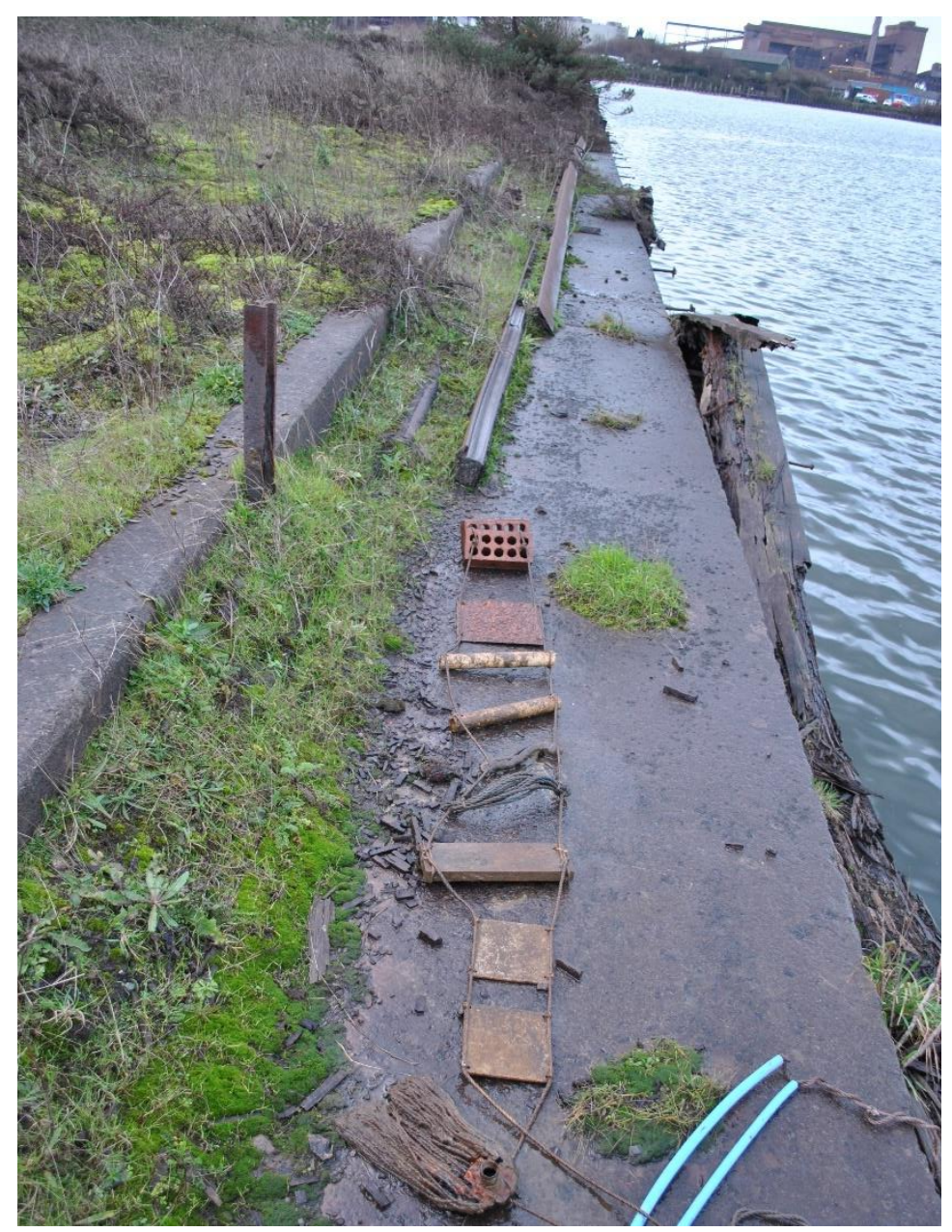




\subsection{Abstract}

Aquatic non-native species (NNS) can pose a significant threat to the environment into which they are introduced. Reducing the risk of NNS introductions can often rely on the intimate knowledge of habitats and environments, as the colonisation by benthic invertebrates can be influenced by a wide range of factors. Understanding how NNS colonise different material types within port environments, where invasion risk is high, may allow for a more targeted management strategy to reduce the impact of NNS introductions. Here, I deployed settlement surveys comprised of 10 different material types within five ports located along the Bristol Channel, South Wales, UK. I report significant differences in whole community composition and total abundance between material types, with PVC, brick and polypropylene rope consistently colonised by a greater number of organisms than other sampled material types. However, NNS were found to consistently colonise all material types. All recorded NNS were previously known to inhabit these ports based on prior surveys. The loss of sample replicates at multiple ports during this study has, however, limited the extent with which the results and observations can be interpreted. I discuss my findings with reference to current NNS management strategies and make suggestions to how port environments should be managed going forward in order to reduce the risk of novel species introductions and reduce the impact of existing NNS.

\subsection{Introduction}

Non-native species (NNS) can have significant impacts on the natural ecosystems into which they are introduced with biodiversity loss, species extinctions, habitat modification, and changes to ecosystem services among the primary concerns when NNS are introduced to a region (Pyšek \& Richardson, 2010; Katsanevakis et al., 2014; Bellard et al., 2016; Gallardo et al., 2016; Walsh et al., 2016). Efforts to mitigate the risk of NNS introductions and to effectively manage NNS once detected are key factors in minimising the potential impacts to natural systems (Blackburn et al., 2011; Giakoumi et al., 2019). 
Effective management of terrestrial NNS relies on the early detection of a novel species introduction to a region which allows for the potential eradication of the species prior to it becoming established and spreading into adjacent regions (Blackburn et al., 2011). Management of aquatic NNS is also most effective following an early detection of a species introduction, however, the numerous transport vectors, large potential area for invasion and reproductive biology of aquatic species makes complete eradication a rarely successful management option (Epstein et al., 2018; Giakoumi et al., 2019). Most strategies therefore focus on the management of impacts of individual species or groups of similar species that are known to be present within a habitat or region (UK Government, 2015; Welsh Government, 2017; Giakoumi et al., 2019). Risk mitigation, on the other hand, is a more generalised approach aimed at reducing the chance of NNS being introduced and can rely on the intimate knowledge of a specific habitat and/or NNS to best understand how to minimise the risks of species introductions (Boon et al., 2020).

We know that the formation of fouling communities and colonisation by NNS can be influenced by a wide range of factors including habitat type (Leclerc and Viard, 2018; Leclerc et al., 2019), predation (Nydam and Stachowicz, 2007; Giachetti et al., 2019), and substrate orientation (Glasby, 2000; Glasby and Connell, 2001), among others (Dobretsov et al., 2005; Crooks et al., 2011; Lindeyer \& Gittenberger, 2011; Brown et al., 2018). The focus of this study will be on substrate type. Substrate type has been previously determined to play an important role in structuring fouling communities, and the differences in communities between different substrates are well documented (Connell, 2000; Lam et al., 2009). This is also the case when focussing on NNS, where it has been suggested that artificial substrate types support an increased number of NNS compared to surrounding natural areas (Tyrrell \& Byers, 2007; Dafforn et al., 2009; Leclerc et al., 2019; O’Shaughnessy et al., 2020).

Research on substrate type and NNS colonisation has focussed on large artificial structures, such as jetties, pontoons, walls and vessels which may be associated with ports and marinas (Mineur et al., 2012; Marraffini et al., 2017; Leclerc et al., 2019). Ports, however, are generally highly industrial areas with a wide range of different materials in the water, which may serve as viable surfaces for the colonisation of NNS. 
Ports are also high-risk environments for the introduction of aquatic NNS due to the close links with the global maritime network, which is widely understood to be the primary vector for aquatic NNS transport (Ruiz et al., 1997; Katsanevakis et al., 2013; Seebens et al., 2013; Williams et al., 2013; Bailey, 2015). Given that NNS management strategies become time-consuming and costly once a species becomes established (Hulme, 2009; Simberloff et al., 2013; Courtois et al., 2018), it is important to target high-risk locations for novel introductions and implement sufficient risk mitigation measures (Katsanevakis et al., 2013; Williams et al., 2013). Better understanding how NNS colonise different materials that are commonplace within port environments may allow for more successful biosecurity measures to be implemented.

Common methods of detecting NNS within communities present on different substrata and material types are Rapid Assessment Surveys (RAS; Arenas et al., 2006; HELCOM, 2013; Bishop et al., 2015; Cook et al., 2015; Rohde et al., 2017). RAS focus on surveying materials and structures that have existed within an environment for a considerable length of time, which allows established communities to be documented without the need to construct, deploy and wait for colonisation of materials (Mineur et al., 2012). Port environments do however present difficulties for the use of RAS. Many RAS rely on being able to access materials exposed during low water, rely on access to floating structures, or use diver or snorkel survey techniques to access submerged materials (e.g. Arenas et al., 2006; Cohen et al., 2005; Collin et al., 2015; Rohde et al., 2017). These methods would not be feasible in most ports due to the use of locks to maintain water levels and safety protocols limiting the presence of people in the water and access to floating or submerged structures (ABP, personal communication). An alternative survey strategy, encompassing aspects of RAS and conventional settlement surveys, has been previously trialled successfully within an active port environment (Holmes and Callaway, 2020), and can be used to compare macrofaunal colonisation of a number of different materials. 
This study aimed to identify how NNS colonise different materials within different port environments with a view to better understanding the attractiveness of materials for fouling species and potential high-risk sites within ports for the colonisation of NNS. Achieving this will allow for more specific biosecurity measures to be implemented within different port environments. This study had the following objectives:

1. Deploy the mixed material survey method as outlined by Holmes and Callaway (2020) in five ports in South Wales, UK

2. Quantify macrofaunal colonisation across 10 different materials types within these five ports

3. Compare NNS colonisation among different material types

\subsection{Materials and methods}

\subsubsection{Study area}

Field surveys were conducted in five active ports: Newport, Cardiff, Barry, Port Talbot, and Swansea, each located along a 55-mile stretch of coastline on the Bristol Channel in South Wales, UK (Figure 21). These ports are all operated by Associated British Ports (ABP). Water levels within each port are maintained to at least $10 \mathrm{~m}$ depth, however environmental conditions vary from port to port. Salinity ranges vary between ports based on measurements taken using an Aquaread AP-5000 probe as well as long term temperature measurements using Tintyag sensors (Table 4). Temperature varies seasonally, however waters in Port Talbot are consistently warmer than any other port due to artificial warming through industrial processes associated with an adjacent steel works. 
Table 4. Salinity and temperature ranges present in each of the five studied ports.

\begin{tabular}{lll}
\hline Port & Salinity range & Temperature range $\left({ }^{\circ} \mathrm{C}\right)$ \\
\hline Newport & $8-16$ & $4.0-21.2$ \\
Cardiff & $1-12$ & $6.6-19.2$ \\
Barry & $22-24$ & $5.4-22.2$ \\
Port Talbot & $0.1-0.3$ & $8.2-25.1$ \\
Swansea & $28-30$ & $5.3-23.8$ \\
\hline
\end{tabular}

Surveys of fouling communities have been previously conducted within these ports and I therefore have a good understanding of the colonising organisms and nonnative species (NNS) that I expect to observe within this study (Naylor, 1957, 1965; Holmes and Callaway, 2020; Holmes and Callaway, 2021).
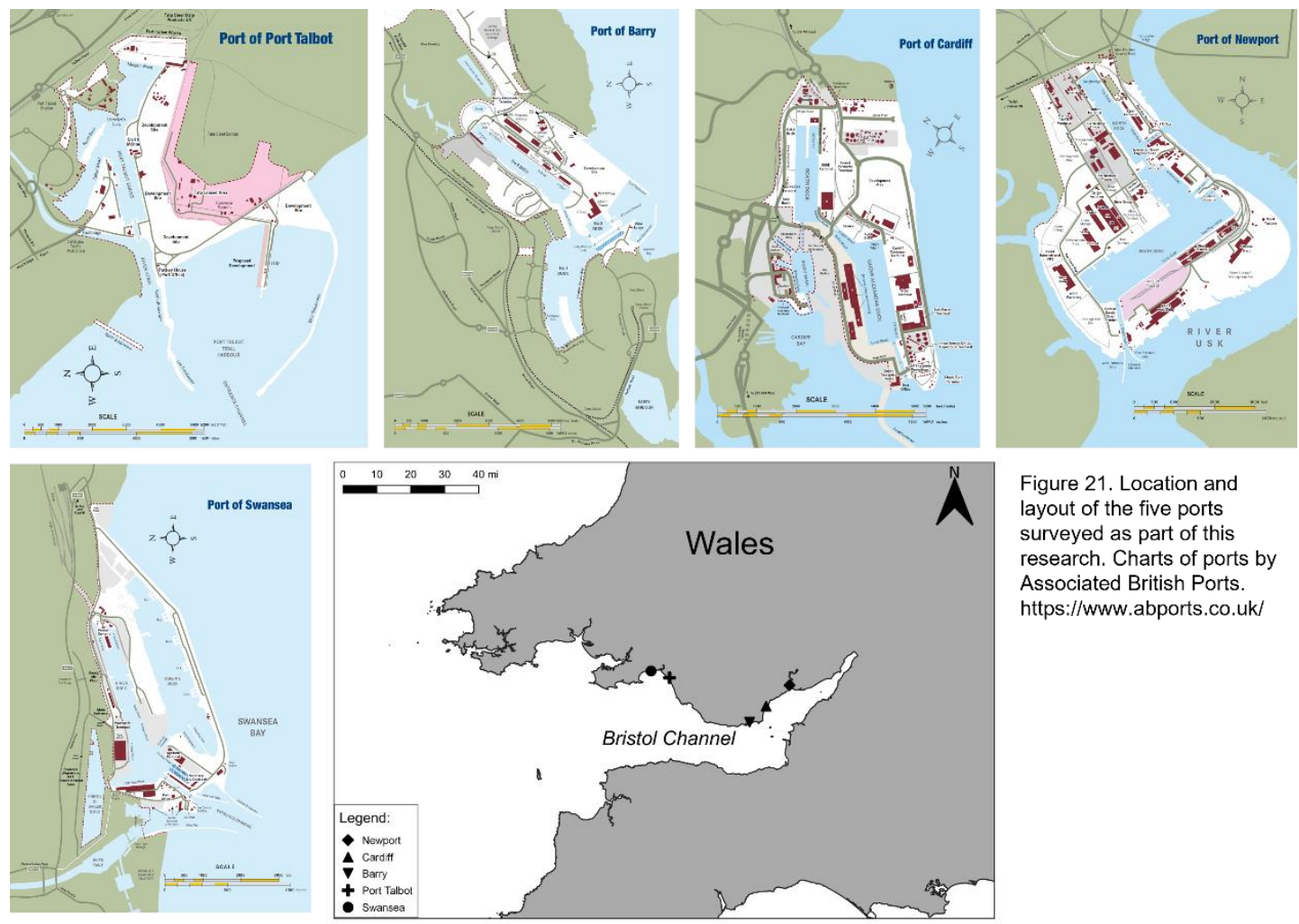

Figure 21. Location and layout of the five ports surveyed as part of this research. Charts of ports by Associated British Ports. https://www.abports.co.uk/ 


\subsubsection{Survey design}

Settlement materials consisted of 10 different material types connected in sequence along polypropylene rope (Figure 22). Materials included: PMMA (sanded and unsanded), PVC piping (sanded and unsanded), wood, brick, natural fibre rope, polypropylene rope, steel, and a cotton fibre mop head. These materials represent different habitat types and materials that are commonplace within port environments based on personal observations made during numerous visits to the studied ports. Additional materials regularly found within ports that were not included here for practical reasons include concrete, natural rock (boulders) and marine litter of varying base material and complexity.

Survey materials were deployed in December 2018 at three locations within each port and remined in situ for 13 months before being removed in January 2020. Upon removal, materials were photographed and analysed in situ to identify macrofaunal colonising organisms. Abundance counts were taken for all countable organisms and an estimation of area covered $\left(\mathrm{cm}^{2}\right)$ was recorded for non-countable, colonial organisms. Where necessary, samples were removed and taken for laboratory analysis to confirm the in situ identification. Materials were also photographed every $6-8$ weeks throughout the survey period.

A number of survey materials were lost over the course of deployment, reducing the number of replicates in the Port of Newport to 2, and the Port of Cardiff to 1 . All three replicates were successfully retrieved and analysed within the ports of Barry, Port Talbot and Swansea. 


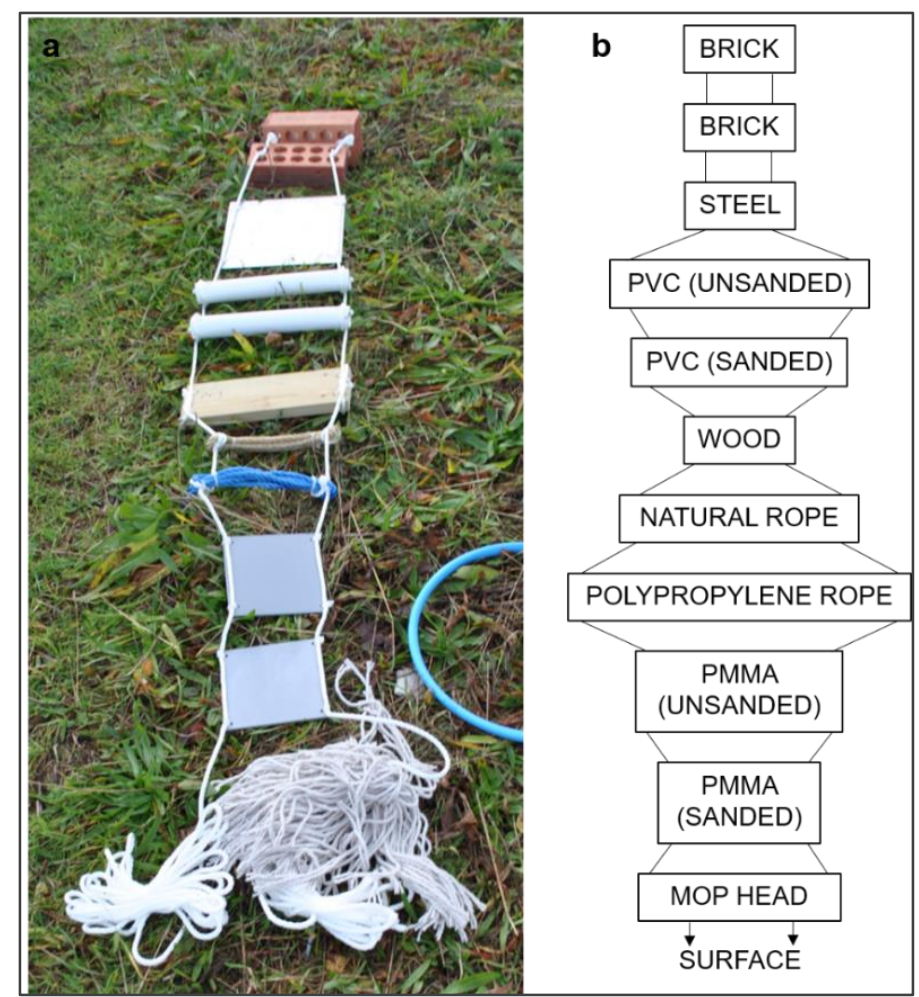

Figure 22. a: Survey materials prior to deployment. b: schematic list of materials in sequence.

\subsubsection{Data analysis}

Abundance counts were combined with coverage data $\left(\mathrm{cm}^{2}\right)$ for those species which could not be counted as individuals. Raw data were then standardised, by the known surface area of each material, to abundance or coverage per $500 \mathrm{~cm}^{2}$. The effect of material type on the observed abundance per $500 \mathrm{~cm}^{2}$ was statistically analysed within R v.3.6.2 (R Core Team, 2017) using a Kruskal-Wallis rank sum test. Post-hoc pairwise Dunn's tests were completed between material groups.

Primer 6 v.6.1.13 with PERMANOVA v.1.0.3 software (Anderson et al., 2008; PERMANOVA+ for PRIMER software) was used to analyse whole community abundance/ coverage and non-native species abundance between samples. Data were first transformed using a square root transformation. A Bray Curtis similarity index was used to create a similarity matrix, from which non-metric multidimensional scaling (nMDS) and PERMANOVA analyses were performed. PERMANOVAs were designed with single factors (Port and Material) and one response variable (values in the similarity matrix). The model used the unrestricted permutation of raw data with type III (partial) sum of squares and 9999 permutations. 
Abundance of NNS observed per $500 \mathrm{~cm}^{2}$ surface area of each material type was also analysed independently within each port. Statistical analysis here was completed within R v.3.6.2 (R Core Team, 2017) and used a Kruskal-Wallis rank sum test with material type as the only factor and abundance per $500 \mathrm{~cm}^{2}$ as the response variable. This analysis was only carried out for data collected within the ports of Newport, Barry, and Swansea due to a lack of replicates from the Port of Cardiff and no NNS reported within the Port of Port Talbot.

Further analysis of different aspects of material colonisation within ports was also completed within Primer 6 v.6.1.13 with PERMANOVA v.1.0.3 (Anderson et al., 2008). Only two ports, Barry and Swansea, were selected for this analysis due to the loss of samples in the ports of Newport and Cardiff and a species-poor environment in the Port of Port Talbot. The effect of material type on whole community composition and total abundance was analysed. Similarity matrices were created using a Bray-Curtis index (whole community) and Euclidian distances (total abundance) based on either square root transformed abundance data (whole community) or raw abundance data (total abundance), from which PERMANOVAs were carried out with the single factor 'Material'. The model used the unrestricted permutation of raw data with type III (partial) sum of squares and 9999 permutations. Pairwise PERMANOVAs were also carried out to test differences between material groups for each measure of colonisation and used the same model design as the main test. Monte-Carlo $\mathrm{p}$ values were used when the number of permutations was low ( $\leq$ 10). It should be noted that a PERMANOVA conducted using one response variable and Euclidian distances (i.e. total abundance per sample) can be used to compute a univariate ANOVA avoiding the assumption of normality (Anderson et al., 2008) as was done in this analysis. 


\subsection{Results}

22 species were identified across the five ports, of which 7 were non-native species (NNS). This is a stark reduction in number of species compared to when using the SSS survey methods outlined in Chapters 1 and 2 due to the lack of laboratory-based processing and identification which enables the detection of more cryptic species. The Port of Swansea had the highest number of species recorded, whilst the Port of Newport had the highest total abundance (full list of species observed available in Supplementary Table S4). Only one species, Fredericella sultana, was recorded from within the Port of Port Talbot. Of the 7 NNS identified 5 may be classified as sessile benthic filter feeders, and these 5 species accounted for $98 \%$ of the total recorded abundance of NNS across all ports (Table 5). Sampling photos available in Appendix III.

Abundance of organisms observed on each material type, pooled across all five ports, was standardised by surface area to the abundance per $500 \mathrm{~cm}^{2}$ (Figures 23 and 24). Material type was found to significantly influence the total abundance of organisms present per $500 \mathrm{~cm}^{2}$ of surface area (Kruskal-Wallis, $\mathrm{p}=0.00003$ ). Pairwise tests revealed significant differences in observed abundance between 15 material group pairings (Table 6; Dunn's test, $\mathrm{p}<0.05$ ). No significant association between material type and the abundance of NNS per $500 \mathrm{~cm}^{2}$ surface area was detected (Kruskal-Wallis, $\mathrm{p}=0.52$ ). 


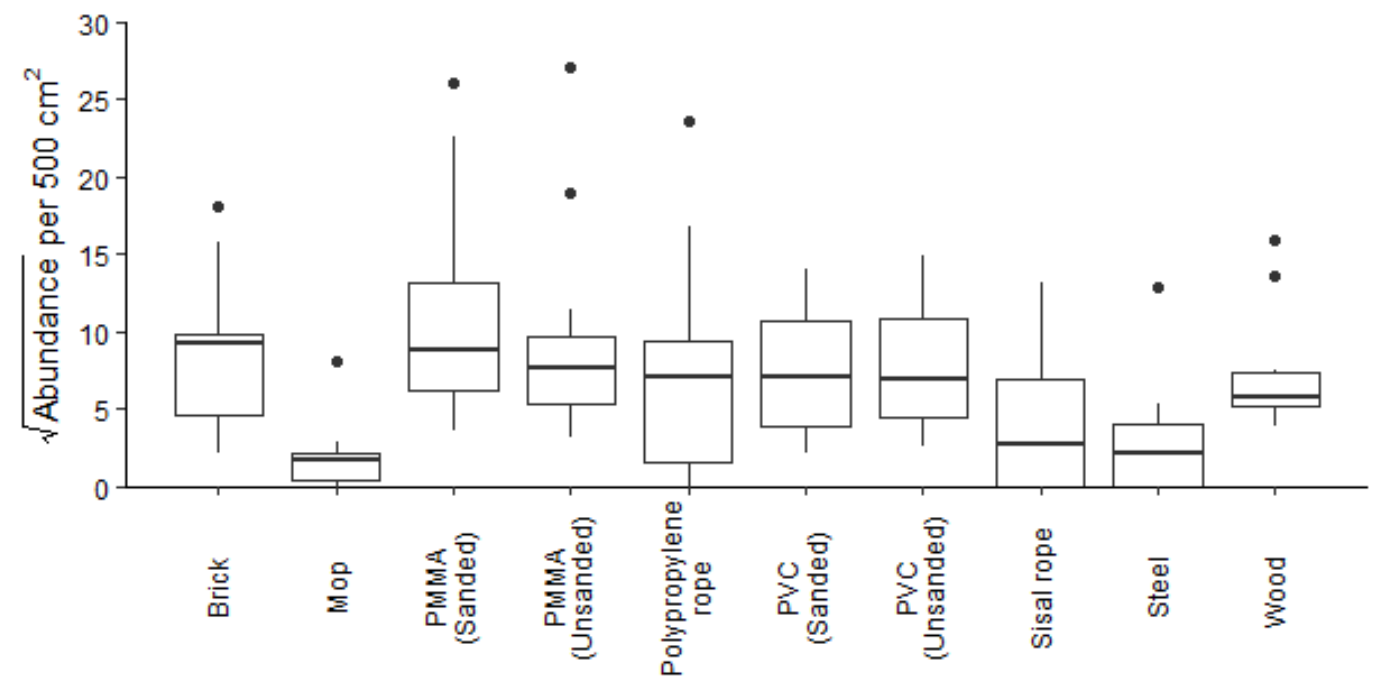

Figure 23. Square root abundance of all recorded organisms per $500 \mathrm{~cm}^{2}$ of surface area for each material group. Data pooled across all ports. $n=12$.

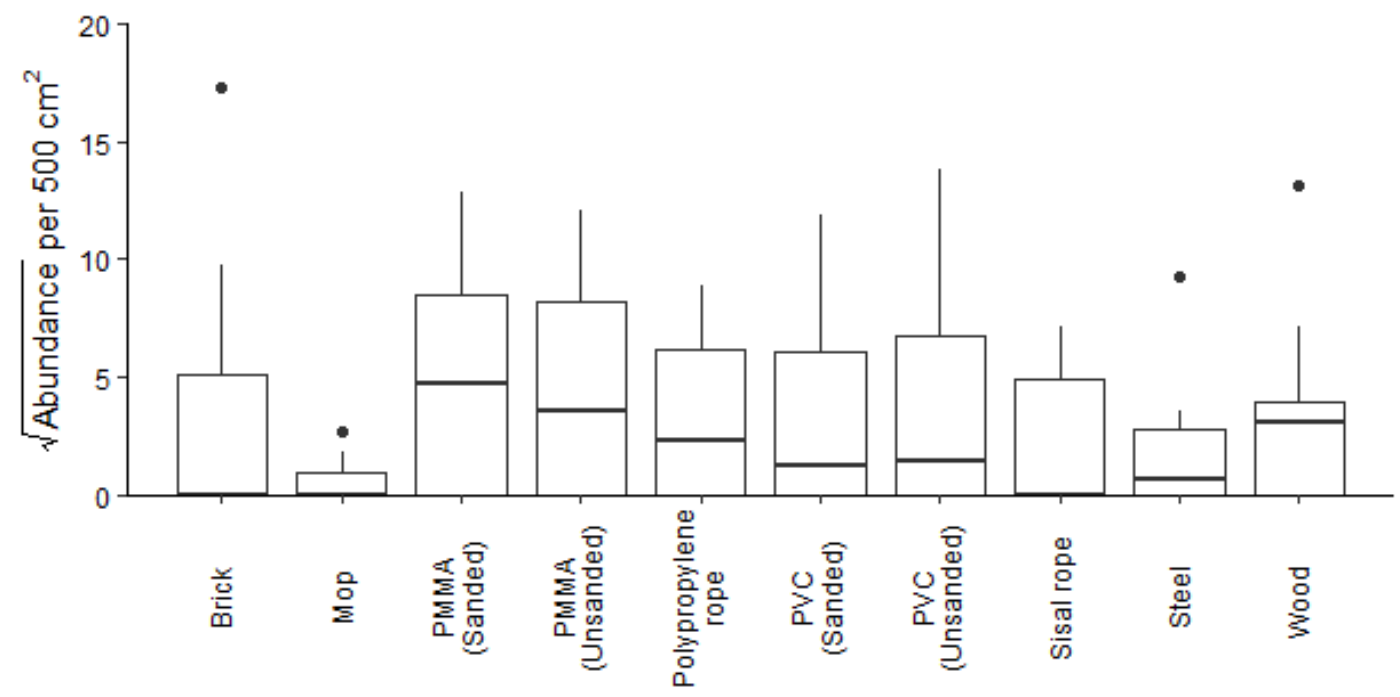

Figure 24. Square root abundance of non-native species per $500 \mathrm{~cm}^{2}$ of surface area for each material group. Data pooled across all ports. $\mathrm{n}=12$. 
Table 5. Total abundance of non-native species observed within each surveyed port. B. stolonifera counted as separate erect colonies. F. enigmaticus counted as individuals.

\begin{tabular}{llllllll}
\hline $\begin{array}{l}\text { Port (n } \\
\text { replicates) }\end{array}$ & $\begin{array}{l}\text { Amphibalanus } \\
\text { improvisus }\end{array}$ & $\begin{array}{l}\text { Austrominius } \\
\text { modestus }\end{array}$ & $\begin{array}{l}\text { Brachynotus } \\
\text { sexdentatus }\end{array}$ & $\begin{array}{l}\text { Bugulina } \\
\text { stolonifera }\end{array}$ & $\begin{array}{l}\text { Caprella } \\
\text { mutica }\end{array}$ & $\begin{array}{l}\text { Ficopomatus } \\
\text { enigmaticus }\end{array}$ \\
\hline Newport (2) & 2301 & - & - & - & - & 23 \\
Cardiff (1) & 407 & - & - & - & - & 562 & - \\
Barry (3) & - & - & - & - & 86 & 176 & - \\
Port Talbot (3) & - & - & - & - & - & - \\
Swansea (3) & - & 156 & 1 & 449 & - & - \\
\hline
\end{tabular}

Table 6. Pairwise comparisons between total abundance of fauna colonising different materials in ports, pooled across all five ports (Dunn's tests). Bold p-values indicate significant differences at the $95 \%$ significance level.

\begin{tabular}{llllllll}
\hline & Brick & $\begin{array}{l}\text { PMMA } \\
\text { (Sanded) }\end{array}$ & $\begin{array}{l}\text { PMMA } \\
\text { (Unsanded) }\end{array}$ & $\begin{array}{l}\text { Polypropylene } \\
\text { rope }\end{array}$ & $\begin{array}{l}\text { PVC } \\
\text { (Sanded) }\end{array}$ & $\begin{array}{l}\text { PVC } \\
\text { (Unsanded) }\end{array}$ & Wood \\
\hline Mop & $\mathbf{0 . 0 0 0 2}$ & $\mathbf{0 . 0 0 0 0}$ & $\mathbf{0 . 0 0 0 1}$ & $\mathbf{0 . 0 0 4 5}$ & $\mathbf{0 . 0 0 0 5}$ & $\mathbf{0 . 0 0 0 6}$ & $\mathbf{0 . 0 0 1 2}$ \\
Sisal & $>0.05$ & $\mathbf{0 . 0 0 2 0}$ & $\mathbf{0 . 0 1 1 9}$ & $>0.05$ & $>0.05$ & $>0.05$ & $>0.05$ \\
rope & & & & & & & \\
Steel & $>0.05$ & $\mathbf{0 . 0 0 0 1}$ & $\mathbf{0 . 0 0 1 0}$ & $\mathbf{0 . 0 1 8 9}$ & $\mathbf{0 . 0 0 3 0}$ & $\mathbf{0 . 0 0 3 4}$ & $\mathbf{0 . 0 0 6 1}$ \\
\hline
\end{tabular}


Whole community analysis revealed 'Port' to be significant factor in determining the observed community composition (PERMANOVA, $\mathrm{p}=0.0001$ ). The similarity in whole community composition between samples from the same port rather than the material type, was visualised by MDS (Figure 25). Material type was not found to significantly influence the observed community (PERMANOVA, $\mathrm{p}=$ 0.7216). This was also the case when considering only the NNS present (PERMANOVA; factor 'Port', $\mathrm{p}=0.0001$; factor 'Material', $\mathrm{p}=0.9774$ ).

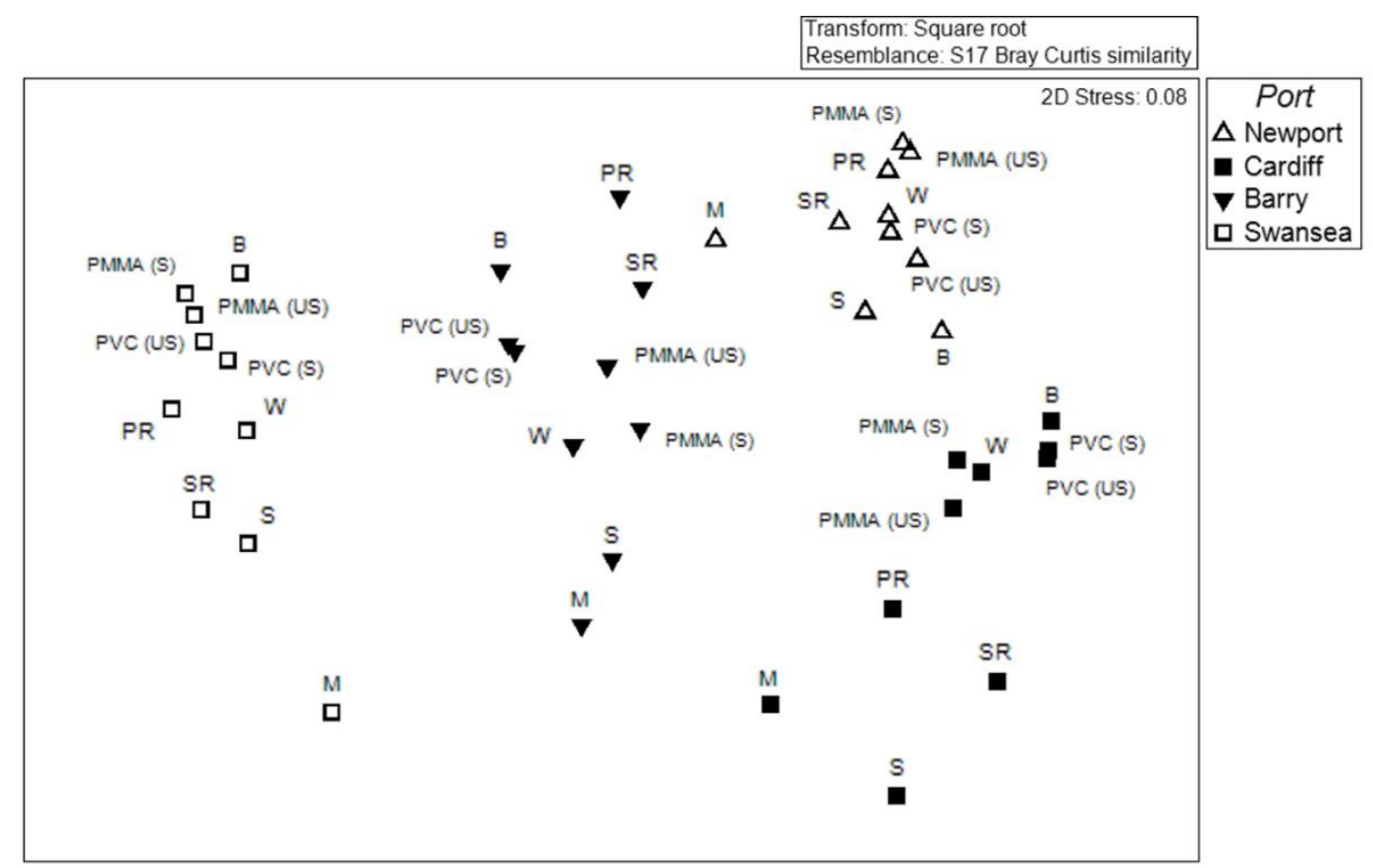

Figure 25. nMDS plot of community samples recorded on 10 different material types within four surveyed ports. Plot based on a similarity matrix created using Bray-Curtis index of square root transformed abundance/ coverage data for each sample. Samples labelled by factor 'Material': $B=$ Brick, $M=$ Mop, PMMA (S) = Sanded PMMA, PMMA $($ US $)=$ Unsanded PMMA, PR = Polypropylene rope, PVC $(\mathrm{S})=$ Sanded PVC, PVC $($ US $)=$ Unsanded PVC, SR = Sisal rope, $\mathrm{S}=$ Steel, $\mathrm{W}=$ Wood. Symbols represent factor 'Port'. Port Talbot samples removed as they were so species-poor they could not be plotted in a meaningful way. 
NNS colonisation of materials within each individual port was analysed based on the abundance of organisms per $500 \mathrm{~cm}^{2}$ of surface area (Figure 26). A breakdown of species found on each material in each port is presented in Supplementary Table S5. In each of the ports of Newport, Barry, and Swansea the observed abundance of NNS was not significantly different between material types (Kruskal-Wallis test, $\mathrm{p}>0.05$ ). Data from the Port of Cardiff was excluded from statistical analysis due to a lack of replicates.
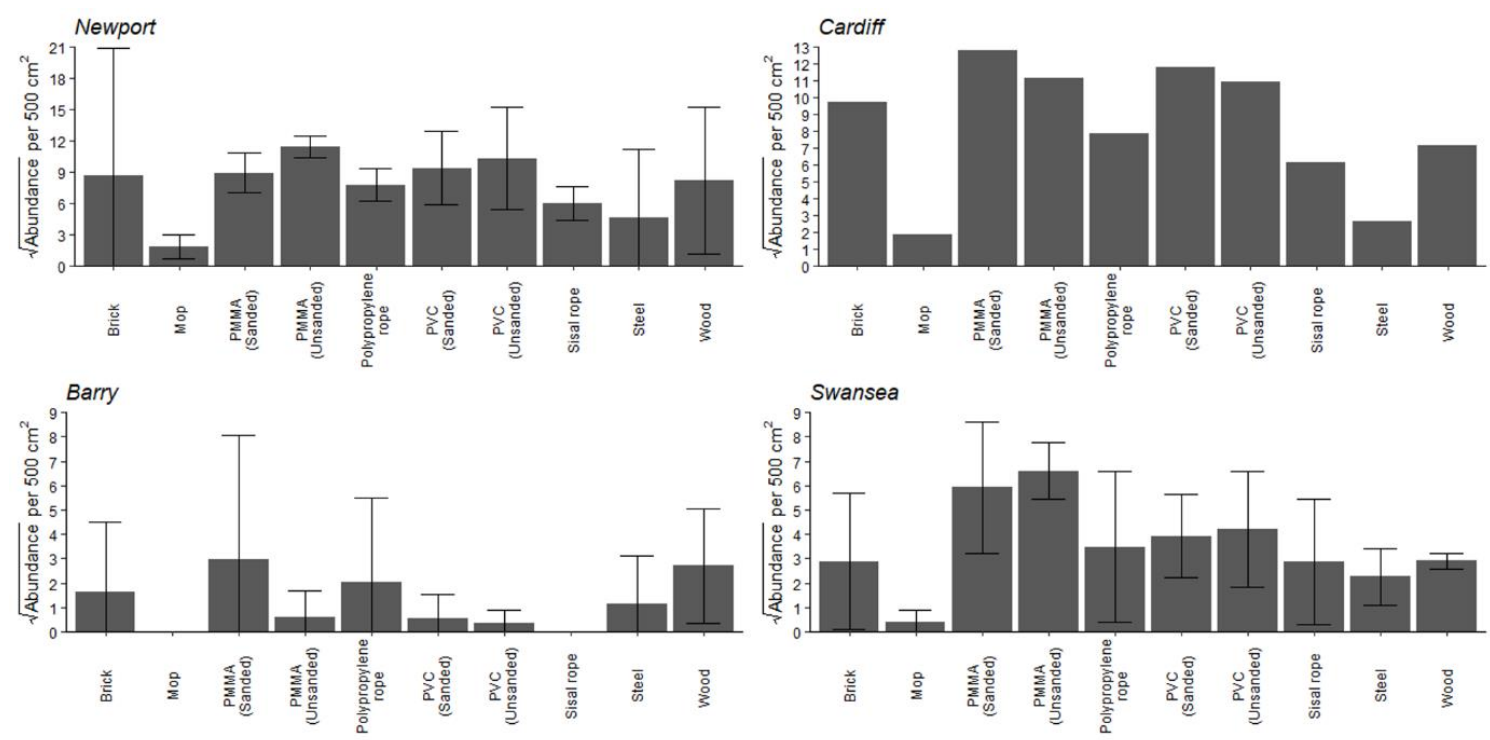

Figure 26. Square root abundance \pm sd of non-natives species (NNS) recorded on each material type in each of four ports: Newport, Cardiff, Barry, and Swansea. No NNS were recorded in the Port of Port Talbot. $n=3$ (Barry, Swansea), $n=2$ (Newport), $\mathrm{n}=1$ (Cardiff).

The proportion of NNS (number of NNS : total number of species) colonising different material types in each port was further analysed, as an indication of the importance the role which NNS may play in the observed fouling communities. Port was once again found to be a significant factor influencing the proportion of NNS within a community (PERMANOVA, pseudo-F $=74.308, \mathrm{p}=0.0001$ ) whilst material type had no such effect (PERMANOVA, pseudo-F = 1.6236, $\mathrm{p}=0.1276$ ). However, the interaction between both factors was found to significantly influence the proportion of NNS in a given fouling community (PERMANOVA, pseudo-F = $1.9418, \mathrm{p}=0.0124)$. 
Since 'Port' as a factor was found to significantly influence observed communities, material type was also analysed as a factor independently within specific ports. Based on the loss of samples during deployment within the ports of Newport and Cardiff, and only one species being recorded within the Port of Port Talbot, this analysis was limited to samples collected within the ports of Barry and Swansea to ensure sufficient replicates of each material group. No measure of colonisation, either whole community composition or total abundance, was found to be significantly influenced by material type within the Port of Barry (Table 7; PERMANOVA, $\mathrm{p}>0.05$ ). Whole community composition and total abundance of organisms were both significantly affected by material type within the Port of Swansea (Table 7; PERMANOVA, p < 0.05). Pairwise analyses reveal significant differences in each measure of colonisation between certain material groups (Table 7). 
Table 7. Results of PERMANOVA main and pairwise tests to determine the effect of material type on whole community composition and total abundance of organisms within the ports of Barry and Swansea. Only material pairs that yielded a significant $p$ value are listed. Significant $p$ values $(\mathrm{p}<0.05)$ are highlighted in bold. $\mathrm{p}$ values for main tests $=$ permutation $\mathrm{p}$, for pairwise tests $=$ Monte Carlo $\mathrm{p}$.

\begin{tabular}{|c|c|c|c|}
\hline & Colonisation measure & $\begin{array}{l}\text { Whole } \\
\text { community }\end{array}$ & $\begin{array}{l}\text { Total } \\
\text { abundance }\end{array}$ \\
\hline & Transformation & Square-root & None \\
\hline & Distance/ similarity & Bray-Curtis & Euclidian \\
\hline Port & Main test/ Material pairs & $\begin{array}{l}p \\
\text { (permutation/ } \\
\text { Monte Carlo) }\end{array}$ & $\begin{array}{l}p \\
\text { (permutation/ } \\
\text { Monte Carlo) }\end{array}$ \\
\hline Barry & Main test & 0.7563 & 0.7835 \\
\hline Swansea & $\begin{array}{l}\text { Main test } \\
\text { Brick \& Mop } \\
\text { Brick \& Polypropylene rope } \\
\text { Brick \& Sisal rope } \\
\text { Brick \& Steel } \\
\text { Brick \& Wood } \\
\text { Mop \& PMMA (Sanded) } \\
\text { Mop \& PMMA (Unsanded) } \\
\text { Mop \& Polypropylene rope } \\
\text { Mop \& PVC (Sanded) } \\
\text { Mop \& PVC (Unsanded) } \\
\text { Mop \& Wood } \\
\text { PMMA (Unsanded) \& Sisal } \\
\text { rope } \\
\text { PMMA (Unsanded) \& Steel } \\
\text { PMMA (Unsanded) \& Wood } \\
\text { Polypropylene rope \& Steel } \\
\text { Polypropylene rope \& Wood } \\
\text { PVC (Sanded) \& Steel } \\
\text { PVC (Sanded) \& Wood }\end{array}$ & $\begin{array}{l}\mathbf{0 . 0 0 0 2} \\
\mathbf{0 . 0 2 3 4} \\
0.1548 \\
0.2371 \\
0.0604 \\
0.1985 \\
\mathbf{0 . 0 2 7 9} \\
\mathbf{0 . 0 1 6 7} \\
\mathbf{0 . 0 2 4 9} \\
\mathbf{0 . 0 1 9 4} \\
\mathbf{0 . 0 1 5 5} \\
\mathbf{0 . 0 1 1 6} \\
0.3149 \\
0.0684 \\
0.0517 \\
0.1732 \\
0.0706 \\
0.1659 \\
0.0919\end{array}$ & $\begin{array}{l}0.0007 \\
0.0003 \\
0.0243 \\
0.0054 \\
0.0008 \\
0.0008 \\
0.0363 \\
0.0005 \\
0.003 \\
0.007 \\
0.0537 \\
0.0037 \\
0.013 \\
0.0017 \\
0.0029 \\
0.0117 \\
0.0262 \\
0.0179 \\
0.0387\end{array}$ \\
\hline
\end{tabular}

Considering the loss of replicates in the ports of Newport and Cardiff as well as the detection of no NNS in the port of Port Talbot, and overall small sample size within port groups (maximum three) thus raising the potential for a Type II error, the results presented in this section should be interpreted with caution. 


\subsection{Discussion}

A wide range of factors are known to influence the settlement of benthic fauna within fouling communities, including abiotic factors such as substrate type, orientation and roughness (e.g. Glasby, 2000; Glasby \& Connell, 2001; Blockley \& Chapman, 2006) and biotic factors such as predation and founder effects (e.g. Nydam \& Stachowicz, 2007; Lindeyer \& Gittenberger, 2011; Vieira et al., 2018; Giachetti et al., 2019). Whole community species composition and abundance of organisms were found to be influenced by material type within this study, when considering data pooled across all surveyed ports. There was, however, no such difference observed in the range of non-native species (NNS), NNS abundance or the proportion of NNS in the community between material types.

The materials used within this study varied in size, shape, roughness, firmness, and complexity. Each of these factors have been previously identified as factors which may influence the formation of fouling communities (Harris and Irons, 1982; Glasby, 1999, 2000; Glasby and Connell, 2001; Leclerc et al., 2019), which aligns with results of this study where communities varied among material types. The fact that the range of NNS and NNS abundance did not significantly differ between material types indicates that the colonisation of NNS was not necessarily determined by the factors which determined the colonisation of many native species. NNS have previously been found to colonise certain materials, substrate types, and habitats in varying abundance, although this has been commonly reported when comparing large scale artificial structures with natural substrata and habitats, as opposed to smaller scale material preferences (Bracewell et al., 2012; Foster et al., 2016; Leclerc and Viard, 2018; Leclerc et al., 2019). NNS are often categorised as opportunist, versatile or resilient species, which are capable of surviving in a range of environmental conditions and often outcompeting the native competitors (Devin and Beisel, 2007; Costello et al., 2015). The consistent colonisation across numerous material types observed here is perhaps therefore linked to the ecological traits of these species which enable them to successfully colonise a wide range of substrate types, whilst the whole community data indicates preferences of species to more 'ideal' substrates which are found to colonise certain materials more densely than others. 
When material type as a factor was assessed independent of the effect of port (Chapter 1), material type was indeed found to significantly influence community colonisation. It is worth noting then that the lack of significance reported here for material type emphasises the importance of port, and therefore environmental conditions, in determining community composition and formation. Notably, five of the seven NNS were functionally similar organisms, equating to $98 \%$ of all NNS abundance: Amphibalanus improvisus, Austrominius modestus, Bugulina stolonifera, Ficopomatus enigmaticus, and Styela clava. These sessile benthic filter feeders occupy a similar ecological niche to one another and are successful NNS worldwide (Bracewell et al., 2012; Porter et al., 2015; Wrange et al., 2016; Lins et al., 2018; Yee et al., 2019). While the identity of the numerically dominant NNS differed among ports based on their environmental tolerances, their functional group remained the same. It appears that this functional group is particularly successful at surviving once introduced to new locations. Further, it may be that this group is better adapted to transport and dispersal over large distances compared with other functional groups. Previous studies of NNS within fouling communities have also reported a high proportion of sessile benthic invertebrates compared to other functional groups (Arenas et al., 2006; Cook et al., 2015; Leclerc et al., 2019). Ship mediated transport of species is the largest vector for NNS worldwide (Katsanevakis et al., 2013; Seebens et al., 2013; Bailey, 2015) and, whilst much of the focus in recent years has been toward the ballast water vector (Bailey, 2015), hull fouling has historically been a significant mode of species transport prior to the increased use of various control measures (Carlton and Hodder, 1995; Gollasch, 2002).

Sessile benthic invertebrates are common hull fouling organisms (Davidson et al., 2009; Coutts et al., 2010; Schimanski et al., 2016) and, since it is likely that the observed NNS were introduced many years ago before measures to manage hull fouling were commonplace (Crooks, 2005), it is perhaps therefore no surprise that this group is the dominant group of NNS observed within these ports. The successful establishment of NNS in some locations with high abundances across a range of different materials, particularly A. improvisus and F. enigmaticus, highlights the importance of early detection and effective management strategies. In this study $F$. enigmaticus and A. improvisus were recorded in high abundance in the ports of Newport and Cardiff, and they were the most abundant NNS across all studied ports. 
Both of these species are commonly associated with biofouling in port and harbour environments around the world (Pernet et al., 2016; Borrell et al., 2017; Charles et al., 2018; Yee et al., 2019), and have been credited with causing economic impacts through the fouling of ships, recreational vessels, power plants, and various cooling mechanisms for industrial processes (Muniz et al., 2005; Streftaris \& Zenetos, 2006; Gren et al., 2009). Ecologically, impacts of these species are more complex. They have been reported to have both positive impact, for example through water purification, and negative impact, for example through interspecific competition (Leppäkoski and Olenin, 2000; Bruschetti et al., 2008; Katsanevakis et al., 2014). F. enigmaticus has indeed been listed as one of the 'worst invasive alien species threatening biodiversity in Europe' (Streftaris \& Zenetos, 2006; EEA, 2007). Whilst both of these species have likely existed within these ports for decades and are present elsewhere in the local region (NBN Atlas, 2020), the abundance with which they have been observed and ability to colonise a wide range of substrates suggests a species-based management strategy may need to be implemented to minimise the risk of further ship mediated spread into other ports and marinas.

The effective management of NNS is a complex process whereby the optimal strategy may depend on a range of factors including the potential effectiveness of the strategy, feasibility of implementing the strategy, social acceptance, any wider impacts, and cost (Hulme, 2009; Blackburn et al., 2011; Giakoumi et al., 2019). There have been recent suggestions to move away from species specific management efforts, which have been frequently used to date (Anderson, 2005; Coutts and Forrest, 2007; Sambrook et al., 2014; Booy et al., 2017), and towards a more generalised approach which would group species with similar characteristics together to determine an optimal management strategy (Giakoumi et al., 2019). This is, however, intended more as a method of speeding up the decision-making process following a novel introduction and not necessarily for use when identifying well established NNS. A species-specific management strategy remains the most effective strategy when targeting established NNS that are potentially highly invasive and damaging organisms (McGeoch et al., 2016; Booy et al., 2017). 
Given the observed readiness with which NNS appear to colonise a range of different material types within these ports, biosecurity measures targeted at reducing NNS abundance and limiting potential future introductions should not focus on any one habitat type. The removal of surplus material from the water should be a priority, including marine litter which can offer a large surface area for colonisation, is a known transport vector for NNS (Gregory, 2009; Miralles et al., 2018; Rech et al., 2018; Agamuthu et al., 2019), and can often aggregate in high density in port environments (pers. observation). Due to the high abundance of sessile benthic NNS, biosecurity protocols should focus on minimising the risk of transmission via shipping through the effective management of the hull fouling and ballast water vectors, in line with International Maritime Organisation (IMO), European Union and national government guidelines (IMO, 2012; Regulation (EU) 1143/2014; Maritime \& Coastguard Agency, 2020). Several of the recorded NNS have indeed been associated with hull fouling of recreational vessels in a number of previous studies (Davidson et al., 2010; Kauano et al., 2017; Peters et al., 2019).

It should be noted that depth is a known factor that can influence the colonisation of benthic fauna and subsequent formation of benthic communities (Hurlbut, 1991; Head et al., 2004; Kazmi et al., 2020). Given that materials deployed here extended over approximately $2-3 \mathrm{~m}$ and water levels were not consistent either over time within ports or between ports it is possible that depth may have influenced the observed colonisation of materials. In future, materials should be randomised in sequence in an attempt to overcome this factor. Surveys could further be adapted by directly targeting the depth factor and deploy materials at set depths (Dafforn et al., 2009; Lezzi and Giangrande, 2018).

Findings of this chapter and particularly the power of comparisons between ports was ultimately hindered by the loss of replicates from three of the five ports (Newport, Cardiff and Port Talbot). Replicates were lost for several reasons ranging from direct removal by persons unknown to the accidental loss through shipping activities (elaborated upon in the General Discussion). Similar monitoring efforts should in the future deploy, where possible, a minimum of five replicates to account for the likely loss of materials. 


\subsection{Conclusion}

Data collected and analysed within this study were ultimately limited by low replication due to the loss of samples in some ports, and all conclusions must therefore be approached with caution. At face value, the range of observed nonnative species (NNS) and abundance of NNS did not differ between material type, whilst total community composition and total abundance were found to vary between materials. This may suggest that NNS are highly adaptable to novel habitats and capable of successfully colonising a wide range of substrates, however more detailed investigation would be needed to confirm this. Sessile benthic filter feeders were the dominant functional group of all recorded NNS which I associate here to the likely introduction via shipping, whereby these organisms are capable of transmission via both the ballast water and hull fouling vectors. Biosecurity management within these ports should not focus on any one habitat type, based on the observed readiness with which NNS will colonise different materials. Management should instead aim to reduce the surplus material within ports, limit the time essential materials spend submerged, and maintain thorough biosecurity checks regarding vessel movements. Future monitoring may take a generalised approach focussing on groups of similar species, however, a species specific management strategy may be necessary in the short-term to minimise the impact and further spread of certain abundant NNS, with a particular focus on Amphibalanus improvisus and Ficopomatus enigmaticus in the ports of Newport and Cardiff. 
CHAPTER 4: Validating the use of plastic settlement tiles in describing fouling communities and identifying non-native species within active port environments

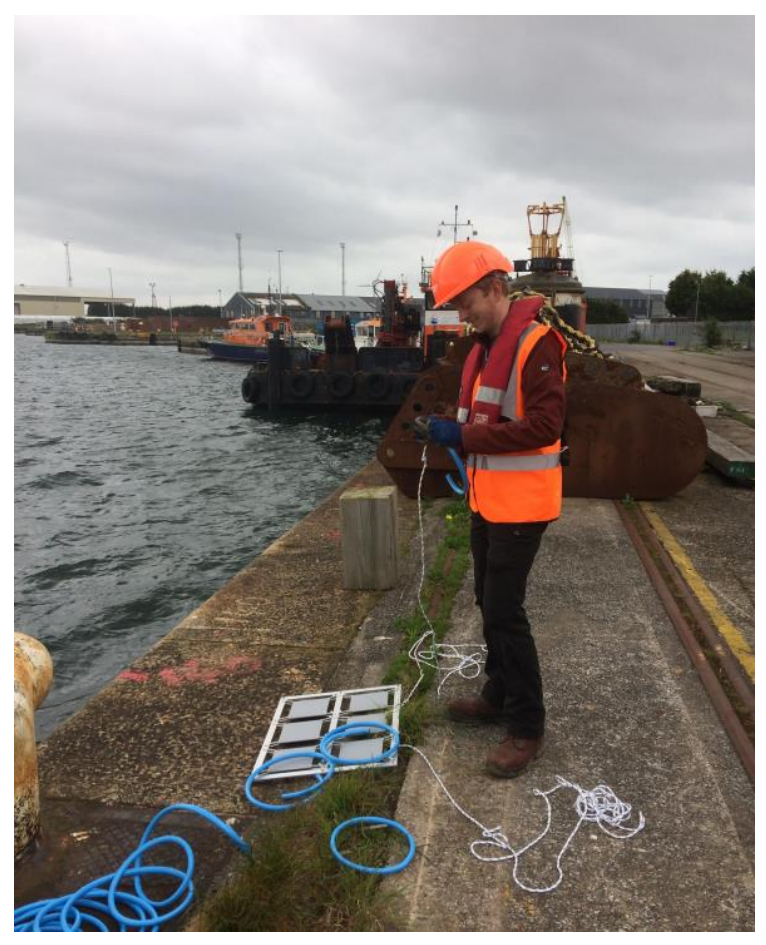




\subsection{Abstract}

Management of non-native species (NNS) is most effective following the early detection of a species post introduction. It follows that monitoring and surveying marine communities for NNS is a key aspect in management efforts, and plastic settlement tiles have regularly been used in these surveys. However, our increased understanding of the potential effects that plastics and plastic leachates can have on the development and survival of marine invertebrates raises the concern that plastic substrates may not provide an accurate representation of the natural fouling community. Here, I deployed two comparable settlement studies utilising both plastic (PMMA) and natural slate substrates within an active port environment. Colonisation of plastic and slate was found to be significantly different in terms of whole community structure, number of species, number of NNS and abundance of NNS. Plastic settlement tiles were colonised on average by a greater number of species (10.3 to 9), a greater number of NNS (2.9 to 2.4), and a greater abundance of NNS (66.6 to 48.1) compared to slate tiles. Loss of sample replicates and the identification of only four NNS within this study does, however, limit the extent with which interpretations of the results and observations can be made. Regarding the use of plastic as a settlement substrate in studies aiming to identify NNS, this method certainly remains a valid survey tool since there is a greater likelihood of identifying more NNS when using plastic settlement tiles as opposed to a natural substrate.

\subsection{Introduction}

The introduction, establishment and spread of marine non-native species (NNS) within a new environment can cause potentially severe environmental and economic impacts (Pyšek \& Richardson, 2010; Simberloff et al., 2013; Katsanevakis et al., 2014; Walsh et al., 2016). NNS are considered one of the greatest threats to the loss of natural biodiversity (McGeoch et al., 2010; Pyšek and Richardson, 2010), can lead to species extinctions (Vitule et al., 2009; Bellard et al., 2016), and can cause significant economic losses either through the direct impact on coastal and maritime industry or through the associated costs of managing species (Pimentel et al., 2001; Lovell and Stone, 2005; Pyšek and Richardson, 2010). 
Management of NNS is a key priority in the effort to reduce the risk of species introductions and minimise the impact post introduction (Coutts \& Forrest, 2007; Pyšek \& Richardson, 2010; Booy et al., 2017; Epstein et al., 2018; Giakoumi et al., 2019). Management of NNS is most effective following the early detection of a novel species introduction, whereby eradication is the ideal management goal (Blackburn et al., 2011; Giakoumi et al., 2019). Continued monitoring of NNS is therefore important in the overall management strategy (UK Government, 2015) and there should be a focus on monitoring areas, regions, and habitats that are at higher risk of novel introductions.

Maritime trade is the primary vector for the spread of marine NNS worldwide (Katsanevakis et al., 2013; Seebens et al., 2013, 2016; Williams et al., 2013; Bailey, 2015), with an early, and no doubt now outdated, estimated 3000 species being transported per day in ballast water alone (Carlton and Geller, 1993). Ports are central to the global maritime trade network and are widely regarded to be some of the most 'at-risk' areas for species introductions (Seebens et al., 2013; Bailey, 2015). A wider concern surrounding ports is the threat that these sites may pose to surrounding habitats and ecosystems by acting as stepping stones for NNS to enter a region before dispersing beyond the port boundary (Floerl et al., 2009). NNS within ports are most commonly associated with fouling communities, with NNS regularly being identified from within communities living on submerged structures and vessel hulls (Arenas et al., 2006; Mineur et al., 2012; Collin et al., 2015; Giachetti et al., 2019).

A commonly used survey method for studying fouling communities and identifying NNS is the use of a settlement survey, which frequently adopt the use of plastic as the settlement surface upon which the colonisation of organisms is recorded (e.g. Sutherland \& Karlson, 1977; Glasby, 1999; Floerl et al., 2012; Bangor University, 2015; Marraffini et al., 2017). There is, however, increasing evidence and concern over the effects that plastics may have on the larval settlement and development of many marine invertebrates (Andrady, 2011; Cole et al., 2011). Nanoand micro-plastics have been found to directly affect various aspects of benthic invertebrate life cycles including mortality, development, behaviour, and reproduction (Au et al., 2015; Martínez-Gómez et al., 2017; Tallec et al., 2018; Haegerbaeumer et al., 2019; Bringer et al., 2020). 
A primary cause for the observed impacts of marine plastics on benthic invertebrates is the toxicity caused by plastic leachates, which has been linked in numerous studies to problems with embryo and larval development (Balbi et al., 2016; Silva et al., 2016; Oliviero et al., 2019; Gardon et al., 2020). Additional problems lie in the ingestion of microplastics which is a particular concern for filter feeding benthic invertebrates and may also impact on the formation of fouling communities through the increased mortality, inhibited food uptake and slower growth rates of adult individuals (Gonçalves et al., 2019; Messinetti et al., 2019). Given the dominance that filter feeders are known to have within fouling communities in the Port of Swansea (Chapters 1,2 and 3) it became apparent that the use of plastic settlement tiles may be negatively impacting communities.

The use of plastic settlement tiles has previously been determined to successfully identify a similar number of NNS within marina fouling communities compared to alternative survey strategies, including diver surveys and rapid assessment surveys (RAS) of existing submerged structures (Marraffini et al., 2017). In doing this, Marraffini et al. (2017) validated the use of plastic settlement tiles as a survey tool to study the colonisation of artificial structures, for example floating pontoons within marinas. However, much of the port infrastructure, whilst being an artificial habitat, is constructed out of materials which are derived from natural components (e.g. bricks) as opposed to plastic based structures such as pontoons. Port walls are one such habitat and can cover a vast surface area viable for colonisation. It follows then that in order to validate the use of plastic settlement tiles as a tool to study colonisation and identify NNS within ports, an alternative study must be conducted.

This study aims to determine whether the colonisation of plastic settlement tiles differs from the colonisation of a natural substrate; in this case represented by natural slate. Particular interest will be paid to the colonisation of these materials by NNS. The study had the following objectives

1. Deploy settlement surveys using PMMA plastic and natural slate tiles within the Port of Swansea.

2. Identify species and quantify colonisation of each material type.

3. Determine the prevalence of NNS on plastic and slate substrates. 


\subsection{Materials and methods}

\subsubsection{Study area}

This study was conducted within the Port of Swansea, South Wales, UK. This is an active port, trading around 600,000 tonnes of cargo a year. Water levels within the port are maintained to at least $10 \mathrm{~m}$ depth and all communities studied here are therefore subtidal. The port is directly connected to the Bristol Channel via a lock which is opened on average around 80 times a month for vessel movements (ABP, unpublished). Salinity levels within the port are consistent within the range of $28-$ 30 with no stratification (Holmes and Callaway, 2020, 2021).

Previous surveys of the fouling community present within this port have identified 50 species, of which 8 can be considered non-native (Holmes and Callaway, 2020, 2021). This port therefore offers an ideal location to analyse the colonisation of natural (slate) and artificial (plastic) substrates by both native and non-native species (NNS) within an active port environment.

\subsubsection{Settlement survey design}

Settlement surveys with two different settlement materials, plastic and natural slate, were used for this study. The surveys deployed here were an extension of the surveys deployed by Holmes and Callaway previously (2020), whereby an aluminium frame is used to house six tiles which serve as virgin settlement surfaces. Two frames were used for each of plastic (PMMA) and slate tiles, totalling 12 replicate tiles of each material. Slate was chosen as a material to represent the natural brick port walls present in much of the Port of Swansea, whilst also being a material which can be engineered to match the dimensions of the PMMA tiles. These frames were deployed at two sites within the Port of Swansea (Figures $27 \& 28$ ) in March 2020 and retrieved in August 2020. Sites were selected based on safety (e.g. proximity to heavy machinery and working berths), accessibility, and availability of a surface mounting point (e.g. mooring bollard or fence).

Tiles were $15 \mathrm{~cm} \times 15 \mathrm{~cm}$ in size and mounted within frames using cable ties. PMMA tiles were lightly sanded using 40 grit sandpaper. For practical reasons of drilling through often fragile slate the slate tiles required a different mounting method to that used for PMMA, but still used cable ties (Figure 28). 
Frames were suspended in the water to a depth of approximately $4 \mathrm{~m}$ using polypropylene rope attached to a fixed surface mounting point (either a mooring bollard or a fence). The water level within the port can vary slightly $(<2 \mathrm{~m})$, meaning that depth would not be consistent throughout the deployment period.

Upon retrieval, settlement tiles were analysed in the field to identify all conspicuous colonising macrofauna to the lowest possible taxon. Data were collected as abundance, for countable organisms, and area covered as an approximation of $\mathrm{cm}^{2}$, for non-countable organisms. Photographs were taken of tiles, from which an overall percentage cover of colonisation was calculated.

An underwater camera rig (Figure 29) was used to capture video footage of the 'natural' port wall as well as each frame housing plastic or slate settlement tiles. Footage was viewed in full and still images extracted, from which fouling species were identified. This survey was designed not to provide a detailed quantified analysis of colonisation between substrate types but instead offer visual 'ground truthing' to the settlement survey.

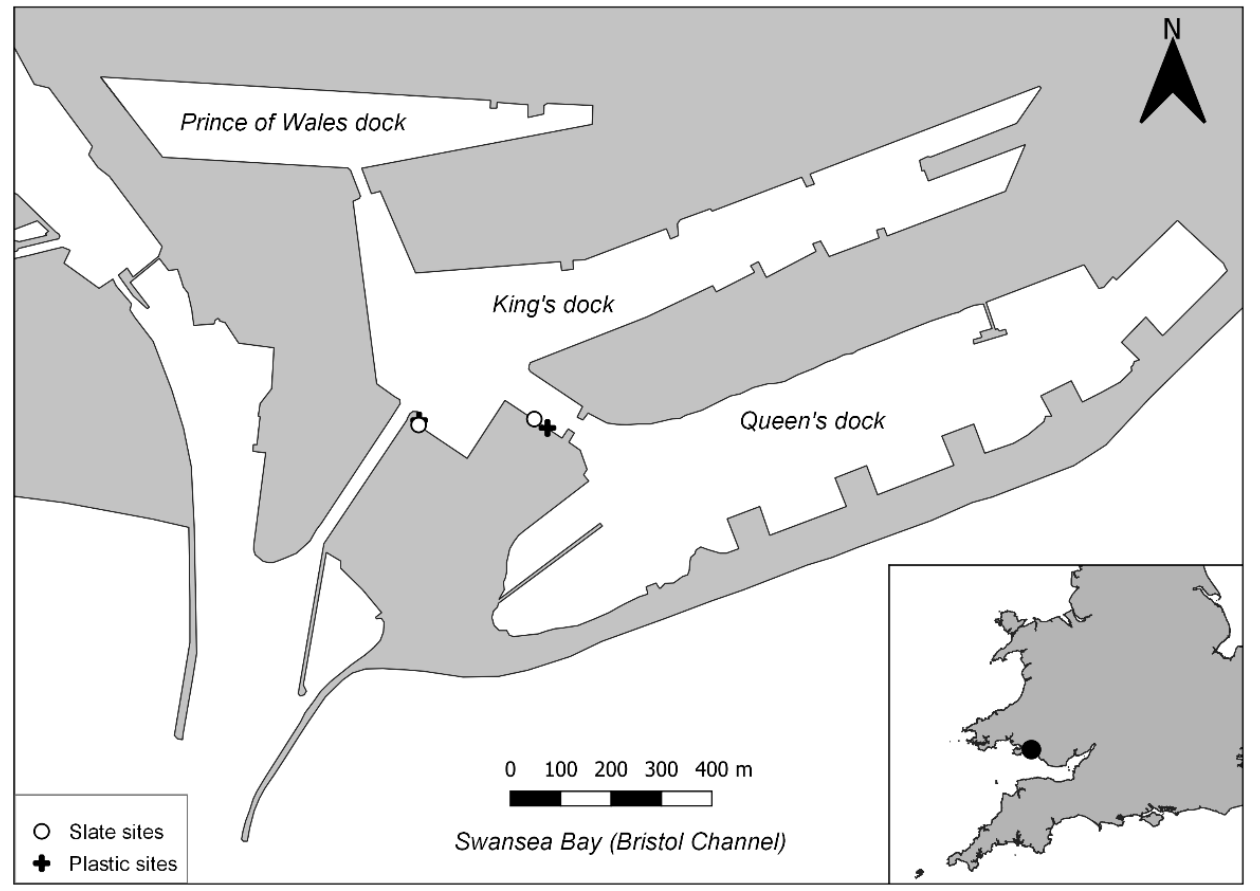

Figure 27. Location of sites for the deployment of survey materials (slate and plastic) within the Port of Swansea. 


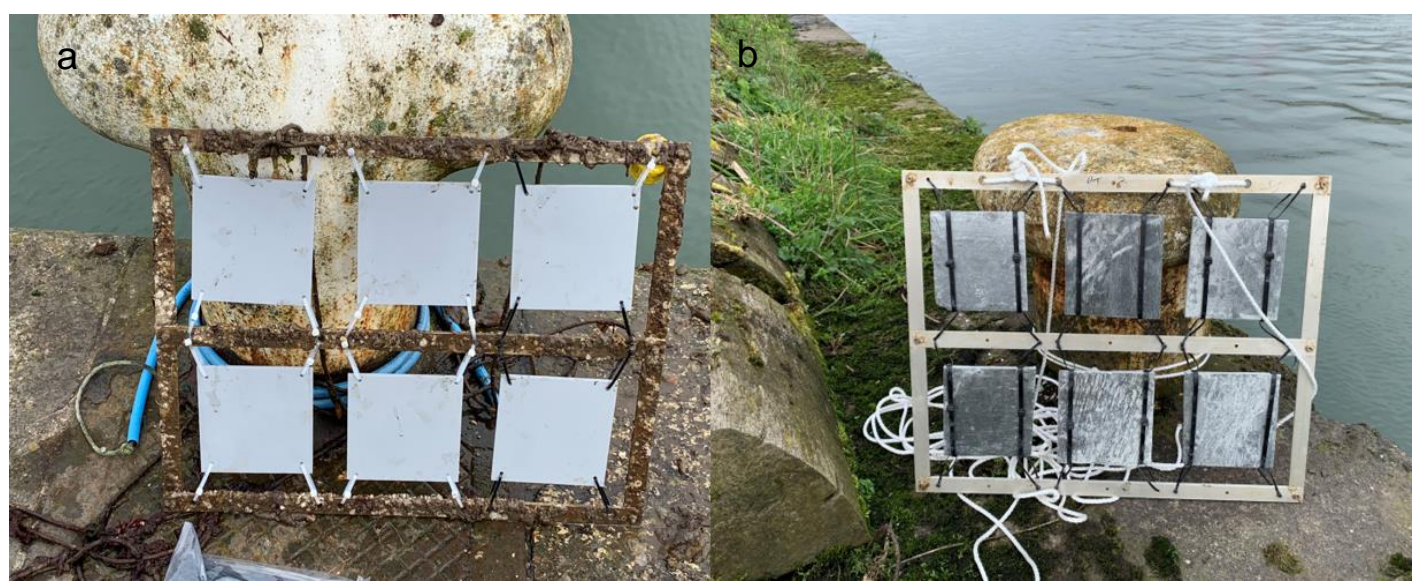

Figure 28. Survey materials prior to deployment. a: PMMA tiles, b: Slate tiles

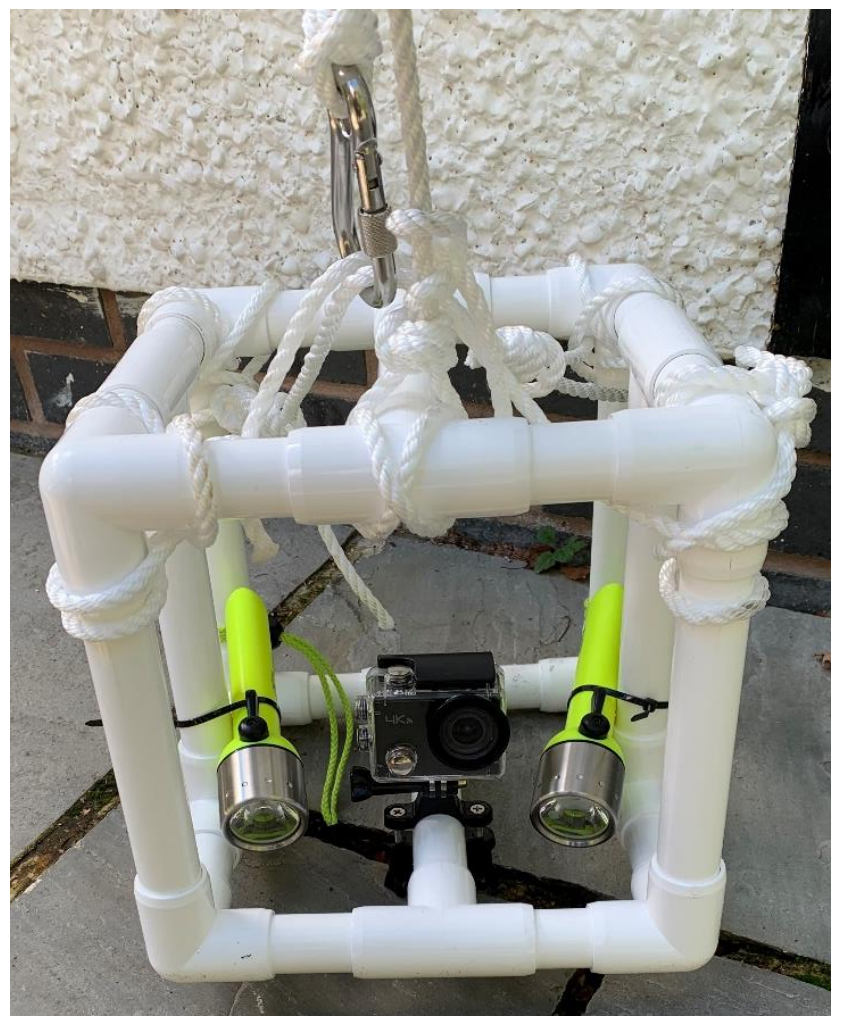

Figure 29. Camera rig used to record images of port wall fouling communities 


\subsubsection{Statistical analysis}

Community analysis was completed within Primer 6 v.6.1.13 with PERMANOVA v.1.0.3 (Anderson et al., 2008) using abundance data and therefore included only countable species. Raw abundance data were $\log (\mathrm{X}+1)$ transformed to downweigh the significance of highly abundant species. A similarity matrix was generated using a Bray Curtis similarity index, from which a PERMANOVA was completed. The PERMANOVA included two factors: 'Material' (two levels: Plastic, Slate) and 'Site' (two levels: site 1 and site 2), and used the permutation of residuals under a reduced model with type III (partial) sum of squares and 9999 permutations. A SIMPER analysis was also conducted using the $\log (\mathrm{X}+1)$ transformed abundance data.

Univariate analysis was completed in R v.3.6.2 (R Core Team, 2017). Failure of normality tests led to the use of Wilcox tests which were used to identify differences in colonisation between slate and plastic tiles based on three univariate measures of colonisation: total number of species, total abundance, and percentage cover. This analysis was also completed for non-native species (NNS), investigating differences in the total number of NNS and abundance of NNS between slate and plastic tiles.

\subsection{Results}

A total of 14 species were identified to species level of which four were nonnative species (NNS; Austrominius modestus, Bugulina stolonifera, Caprella mutica, and Styela clava). 12 species were countable and therefore included in statistical analyses (full species abundance/ coverage data available in Supplementary Table S6). Fouling communities were dominated by the solitary ascidians Ascidiella scabra (total recorded abundance: $30690.99 \mathrm{~m}^{-2}$ ) and Ciona intestinalis (total recorded abundance: $6290.99 \mathrm{~m}^{-2}$ ) as well as the NNS Bugulina stolonifera (total recorded abundance: $11200.99 \mathrm{~m}^{-2}$ ). Eight of the 14 identified species can be classed as sessile benthic filter feeders and account for $99 \%$ of the total recorded abundance or organisms across both substrate types making this functional group the most common within the community. Sampling photos available in Appendix III. 
The difference in whole community composition recorded on each material type and at each sampling site was visualised using an nMDS (Figure 30). Both material type and sampling site were found to significantly influence the observed communities (PERMANOVA: Material, pseudo-F $=6.813, \mathrm{p}=0.0001$; Site, pseudo$\mathrm{F}=11.799, \mathrm{p}=0.0001)$. However, note that the loss of two slate replicates from Site 2 brings into question the reliability of this statistic, and it should therefore be taken with caution. A SIMPER analysis revealed that the species accounting cumulatively for over $90 \%$ of the community dissimilarity between material types were present in communities recorded from both material types (Table 8). The difference in abundance of these species within communities is driving the dissimilarity between material groups, rather than the presence/ absence of species.

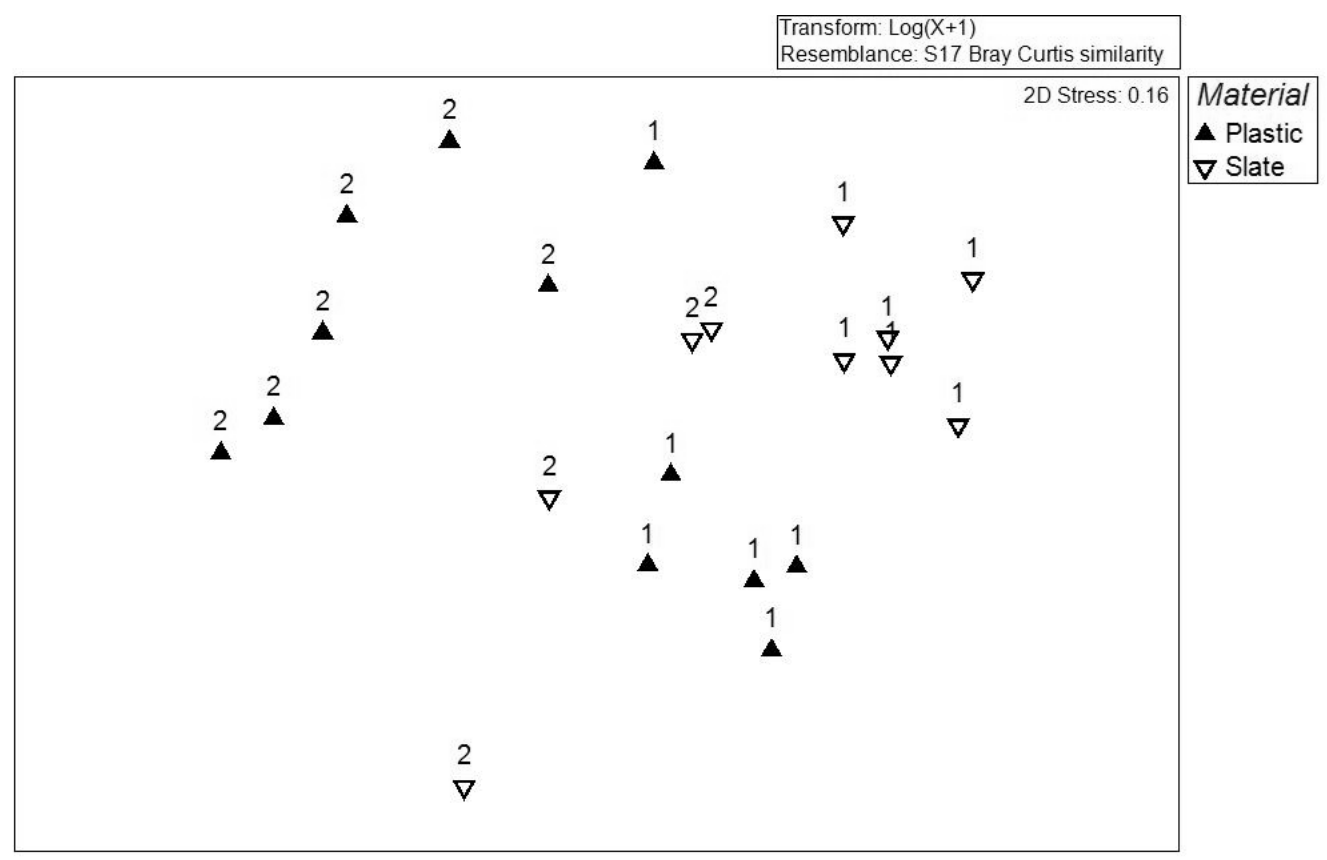

Figure 30. nMDS plot of fouling community samples observed on two material types and at two sampling sites. Data labels indicate sampling site (site 1 or site 2), symbols indicate material type (plastic or slate). Plot based on a resemblance matrix created using a Bray-Curtis similarity index and $\log (X+1)$ transformed abundance count data. 
Table 8. Output data from a one-way SIMPER analysis with the factor 'material type'. Analysis conducted using $\log (\mathrm{X}+1)$ transformed community abundance data for two material types: plastic and slate. Plastic: $\mathrm{n}=12$. Slate: $\mathrm{n}=10$.

\begin{tabular}{llllllll}
\hline & $\begin{array}{l}\text { Av. } \\
\text { Abundance } \\
\text { (Plastic) }\end{array}$ & $\begin{array}{l}\text { Av. } \\
\text { Abundance } \\
\text { Species }\end{array}$ & $\begin{array}{l}\text { Av. } \\
\text { Dissimilarity }\end{array}$ & & Diss/SD & $\begin{array}{l}\text { Contrib } \\
\%\end{array}$ & $\begin{array}{l}\text { Cum. } \\
\%\end{array}$ \\
\hline $\begin{array}{l}\text { Ciona intestinalis } \\
\text { Balanus crenatus }\end{array}$ & 1.92 & 3.22 & 6.21 & 1.39 & 26.88 & 26.88 \\
$\begin{array}{l}\text { Austrominius } \\
\text { modestus }\end{array}$ & 1.92 & 2.70 & 4.16 & 1.85 & 18.00 & 44.87 \\
$\begin{array}{l}\text { Mytilus edulis } \\
\text { Spirobranchus }\end{array}$ & 1.44 & 0.35 & 3.99 & 1.42 & 17.26 & 62.13 \\
triqueter & 1.41 & 1.01 & 2.58 & 1.40 & 11.16 & 73.30 \\
Ascidiella scabra & & & & & & \\
Bugulina stolonifera & 0.68 & 0.25 & 1.78 & 1.31 & 7.69 & 80.98 \\
& 5.07 & 4.62 & 1.66 & 1.24 & 7.18 & 88.16 \\
& 4.05 & 3.88 & 0.72 & 1.42 & 3.10 & 91.26 \\
\hline
\end{tabular}

Further analysis of the differences in colonisation between material types focussed on the number of species and total abundance recorded in samples (Figure 31 ) and the total percentage cover of colonisation. No significant difference was found in the abundance of organisms colonising each material type (Wilcox test, $p>$ 0.05), however, the number of species colonising plastic tiles was significantly greater than that of slate tiles (Wilcox test, $\mathrm{W}=95, \mathrm{p}=0.0169$ ). There were also significant differences in the number of non-native species (NNS) and total abundance of NNS recorded in communities from plastic and slate tiles (Wilcox test: Number of NNS, $\mathrm{W}=93, \mathrm{p}=0.0192$; Abundance of NNS, $\mathrm{W}=101.5, \mathrm{p}=0.0068$ ). In both cases plastic tiles were on average colonised by more NNS and a greater abundance of NNS. However, only four NNS were identified within this study which may reduce the power of this statistic. No significant difference was found in the total percentage cover of colonisation recorded from plastic (mean percentage cover $89.77 \pm 8.78 \mathrm{sd}$ ) and slate tiles (mean percentage cover $87.42 \pm 11.03$ ) (Wilcox test, $\mathrm{p}>0.05)$. As stated previously, the loss of two slate replicates from Site 2 limits the reliability of these statistics and therefore these results should be interpreted with caution.

When considering the proportion of NNS (number of NNS : total number of species) colonising each material type, there was no significant difference observed between plastic or slate substrates (PERMANOVA, pseudo-F $=0.97379, p=0.3451$ ) or between the two sites studied within the port (PERMANOVA, pseudo-F $=4.4701$, $\mathrm{p}=0.0514)$. 
Given the small number of sites studied and loss of two slate replicates from site 2, thus raising the potential for a Type II error, the significance of test results presented here should be interpreted with caution.

Underwater video footage revealed a fouling community present on the 'natural' port walls that was dominated by large solitary ascidians, concurrent with the findings of the settlement study (Figure 32). Clearly visible in the images are Ascidiella scabra, Ciona intestinalis, and Bugulina stolonifera which were the three most abundance species recorded when analysing settlement tiles.

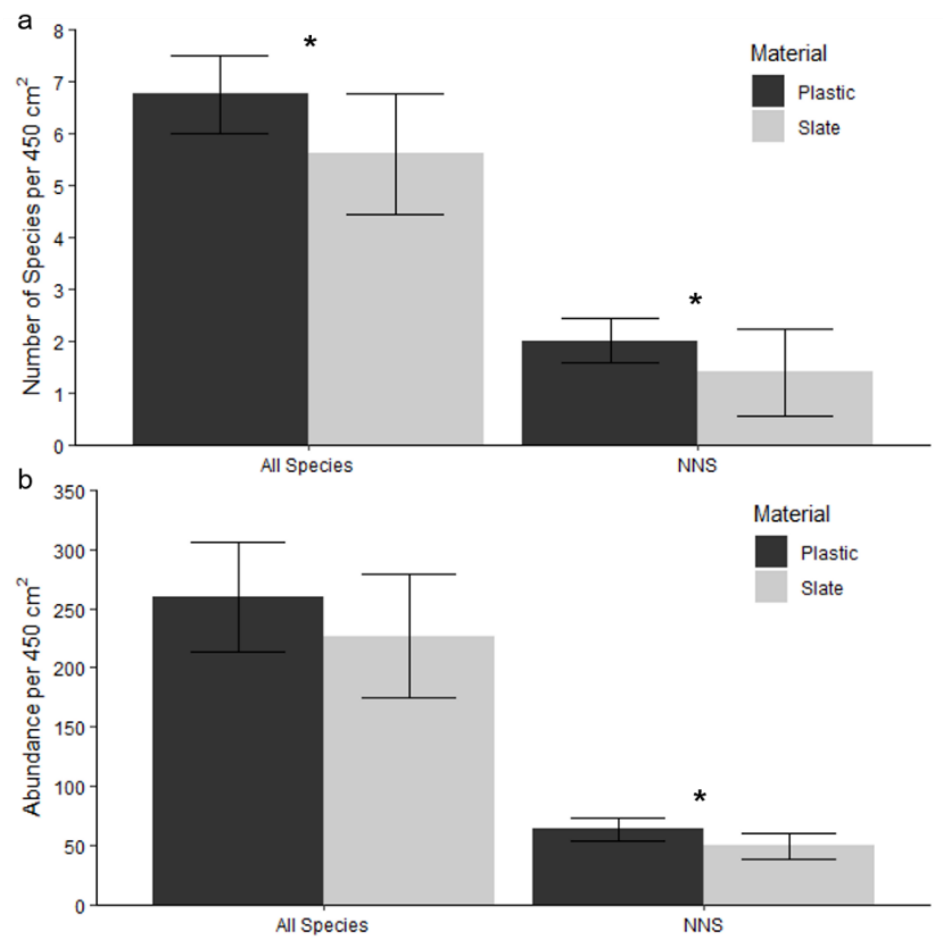

Figure 31. a: Mean number of species $450 \mathrm{~cm}^{-2}$ and $\mathrm{b}$ : mean abundance of organisms $450 \mathrm{~cm}^{-2}$ recorded colonising plastic and slate settlement tiles. Data presented for all documented countable species and for non-native species (NNS) only. Error bars show standard deviation. Plastic: $n=12$. Slate: $n=10$. '*' denote a significant difference in the colonisation of plastic and slate (Wilcox tests, $p<$ $0.05)$. 


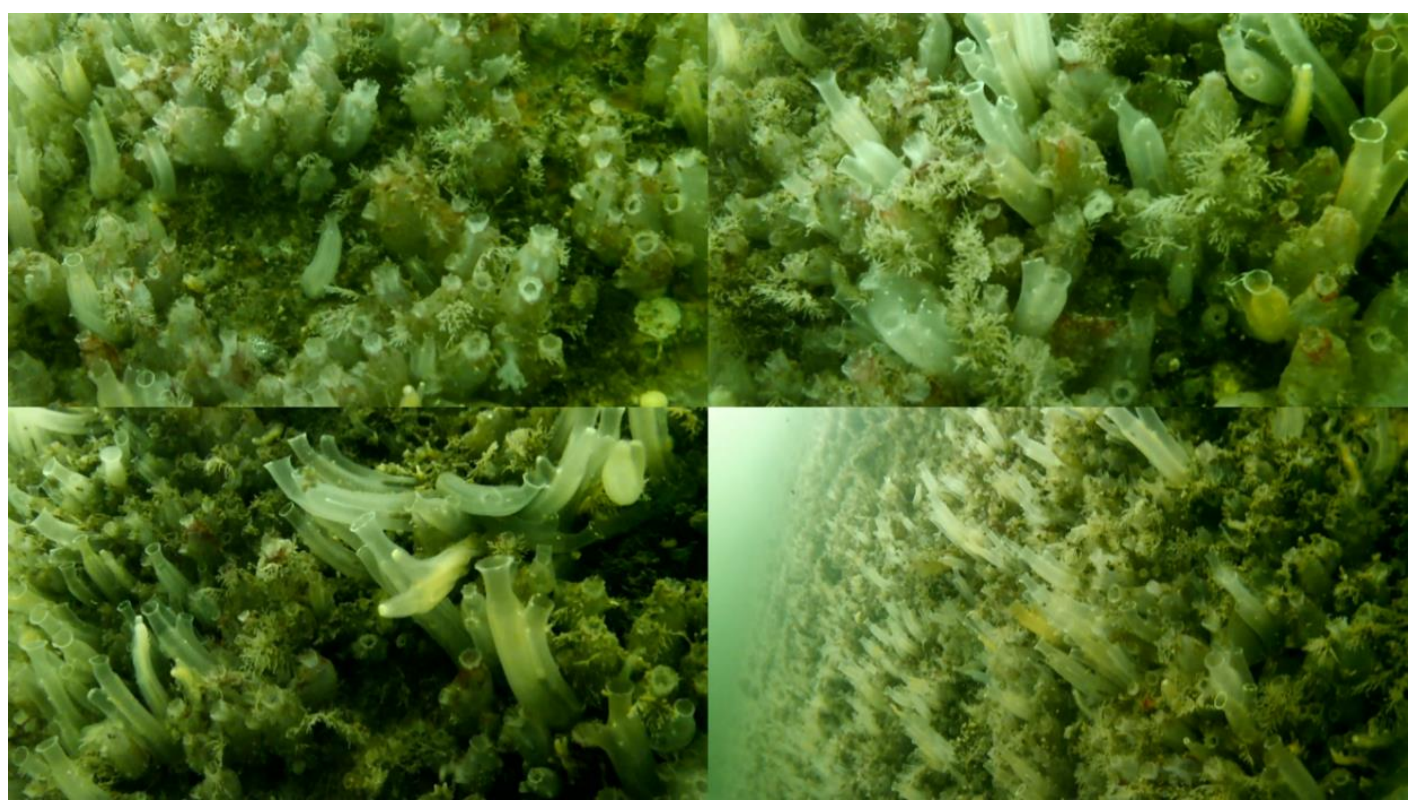

Figure 32. Still images extracted from video footage of port walls.

\subsection{Discussion}

The colonisation of plastics in the marine environment has been well documented in recent years, particularly in reference to the spread of non-native species (NNS) through rafting (Gregory, 2009; Canning-Clode et al., 2013; Rech et al., 2016, 2018; Gündoğdu et al., 2017; Miralles et al., 2018). Whilst plastics will be readily colonised by fouling organisms there has been little research conducted to compare the colonisation of plastics to the colonisation of natural substates in a controlled environment (e.g. Beaumont et al., 2007; Tyrrell \& Byers, 2007). I reported here that a plastic substrate was colonised on average by a greater number of species, a greater number of NNS and a greater abundance of NNS than a natural slate substrate. This is concurrent with several findings reported from a similar study (Beaumont et al., 2007), however Beaumont et al. only considered whole community colonisation and not specifically NNS colonisation.

A primary concern regarding the use of plastic substrates in settlement studies is the effect that microplastics and plastic leachates may have on larval survival, larval recruitment and post-settlement development of individuals (Cole et al., 2011; Silva et al., 2016; Martínez-Gómez et al., 2017; Haegerbaeumer et al., 2019; Oliviero et al., 2019; Gardon et al., 2020). This may lead to the formation of a community that is not representative of the natural community, rendering the use of plastic settlement tiles redundant in many applications. 
It is certainly possible that these effects may have contributed here to the differences observed in whole community composition between plastic and slate substrates. I found differences to stem from relative differences in abundance of species rather than differences in the presence or absence of species between substrate types, so it cannot be discounted that the rates of larval and post-larval survival are influencing these observations

$99 \%$ of the total number of organisms identified were sessile benthic filter feeders, including the three most abundant species Ascidiella scabra, Ciona intestinalis, and Bugulina stolonifera. The ingestion of microplastics by filter feeding organisms has been well documented and found to impede growth rates as well as increase mortality (Gonçalves et al., 2019; Messinetti et al., 2019). It is unlikely that the plastic substrate used in this study has significantly impeded the development of filter feeders, given the high abundance in which this functional group was recorded and the size of individuals. However, since microplastic loading within the port and microplastic ingestion by filter feeders was not directly investigated here the links between plastic ingestion and filter feeders remains broadly observational. There is the possibility that this effect could influence the further development of the fouling community had the settlement tiles remained in deployment for a longer period of time. However, studies investigating the influence of microplastic ingestion on filter feeders are also conducted over a short time period, no more than 42 days of exposure, as the majority of studies focus on larval and juvenile development (Au et al., 2015; Silva et al., 2016; Martínez-Gómez et al., 2017; Gonçalves et al., 2019; Luan et al., 2019; Messinetti et al., 2019; Bringer et al., 2020). Impacts documented from experimental studies may therefore be negated by the influence of water movement, reducing the concentration of microplastics in the vicinity of the settlement tiles. A number of studies have also reported the ability of certain filter feeders to successfully cope with the ingestion of microplastics (Kaposi et al., 2014; Gonçalves et al., 2019; Messinetti et al., 2019). C. intestinalis, which recorded in relatively high abundance in this study, can successfully translocate microplastics out of the gut within hours of ingestion (Messinetti et al., 2019). Messinetti et al. (2019) did however note that growth rate was reduced, likely due to inhibited food intake (Messinetti et al., 2019). 
It remains then that the fouling community present within the Port of Swansea, dominated by solitary ascidians, may be naturally resilient to the presence of plastics and microplastics whereby the same species are able to colonise both natural and plastic substrates. Further investigation, for example transferring colonised plates into a microcosm experiment, would be required to validate or reject this hypothesis.

Whilst there were statistical differences in the colonisation recorded between plastic and slate settlement tiles, the plastic tiles were in each case colonised to a greater degree. The higher average number of species and NNS reported colonising plastic tiles suggests the use of plastic would offer a more effective survey tool than natural materials when attempting to identify NNS within fouling communities. As plastic settlement tiles are largely used in studies aiming to quantify species richness or percentage cover, as opposed to abundances (e.g. Glasby, 1999, 2000; Tyrrell \& Byers, 2007; Bishop et al., 2015; Lezzi \& Giangrande, 2018; Leclerc et al., 2019), the potential difference in the abundance of species colonising plastic tiles compared to a natural substrate is unlikely to impact the research findings. Marraffini et al. (2017) came to a similar conclusion when evaluating the use of settlement tiles in comparison to an alternative survey technique. However, as mentioned when reporting these results, a lack of replication, randomisation and low number of NNS identified in total limits the reliability of the conclusions that may be drawn from the observations reported within this chapter.

As a method of describing fouling communities, settlement tiles offer a range of benefits by comparison to alternative survey techniques. Settlement tiles provide a standardised method whereby findings are comparable across studies, they provide easily quantifiable community data, are often less labour intensive than other survey techniques such as diver surveys, and importantly can capture cryptic species and smaller organisms which may be overlooked in rapid assessment surveys (Marraffini et al., 2017; Rohde et al., 2017). Port environments also limit the use of other commonly used survey techniques such as rapid assessment and diver surveys. The use of video or image analysis cannot provide sufficient detail to accurately describe fouling communities (Beaumont et al., 2007), however the application within this study to offer an element of ground truthing added a degree of confidence in confirming the most dominant species present on port walls. 
Using slate settlement tiles has, to the best of our knowledge, never been done previously in colonisation studies and there are a number of practical aspects to note if considering the use of slate in similar applications. Slate is harder to cut than plastic and weighs considerably more, especially when heavily colonised, to the point that it may cause difficulties in retrieval. Slate is also more likely to break, leading to the loss of samples, and is generally more expensive than plastic alternatives. Slate proved though a viable alternative to plastic when settlement tiles are the only suitable survey option and should be considered in future studies when the research aim does not focus on detecting NNS.

\subsection{Conclusion}

Data collected and analysed within this study were ultimately limited by low replication and limit groups within test factors (i.e. only two groups for each of material and site factors), and all conclusions must therefore be approached with caution. Whilst the colonisation of natural (slate) and artificial (plastic) settlement tiles was different in terms of whole community structure, number of species, number of non-native species (NNS) and abundance of NNS, the use of plastic as a tool in identifying fouling organisms and NNS remains a valid survey approach. At face value, plastic settlement tiles may be more likely to identify a greater number of species and NNS than natural settlement tiles. Plastic substrates may therefore prove a more effective survey tool when the research aim is to identify species or NNS within an environment. If, however, the research aim is to provide the most accurate representation of a particular fouling community, taking into account abundance rather than only species richness, then a natural substrate would be the most appropriate survey tool. 


\section{GENERAL DISCUSSION}


This thesis demonstrates that with the application of tailored survey techniques and a strong working relationship with port operators, effective and informative settlement surveys can be conducted within active ports. The results can significantly increase our knowledge of non-native species from within UK ports and inform bespoke management strategies on a port-by-port basis.

This section will summarise the key themes presented throughout the thesis whilst touching on aspects of the research which were not well placed in any individual chapter.

\section{III.I. Working within active ports}

I have made repeated reference to the importance of ports in the transport of species around the world, which stems almost entirely from the integral role that ports play in the maritime trade industry, the most significant pathway of species introductions worldwide (Carlton and Geller, 1993; Katsanevakis et al., 2013; Seebens, Gastner and Blasius, 2013; Williams et al., 2013). In the General Introduction and within some chapters, I touched on the scarcity of data reporting on non-native species (NNS) from within ports within the UK (section I.VI.I.), making this research rare within the UK. With the exception of the work by Kakkonen et al. (Kakkonen et al., 2019) in Orkney there are, to the best of my knowledge, no established long-term port-based monitoring of NNS within the UK. I believe there are two primary reasons for this: firstly, there is a lack of collaboration between researchers and port operators, which makes ports fairly inaccessible areas; secondly, a lack of instruction or standardised methodology offered to port operators makes it difficult for these organisations to conduct surveys themselves. However, ports are areas of great interest and importance for researchers given the role these areas are likely to play in the spread of NNS. My main objective for this project was to develop a survey methodology specifically designed for use within active ports, which would enable me to address a number of other objectives as well as pave the way for future and more widespread monitoring of NNS within ports. 
Each of the two survey designs implemented as part of this thesis, the Successional Settlement Survey (SSS) and Mixed Material Survey (MMS), have proven effective in the collection of detailed community level data from within active ports. In Chapters 1 and 2 I was able to report how the SSS may be used to achieve three key objectives: 1) to detect NNS, 2) to describe the wider fouling community, including native organisms, and 3) to provide insight into the succession and formation of whole fouling communities. Settlement studies have been used extensively in the past to achieve the first two objectives, although many have focussed exclusively on NNS and paid little attention to the native species (e.g. Arenas et al., 2006; Collin et al., 2015; Bangor University, 2015; Hurst, 2016). However, very few studies that use settlement studies take into account a temporal factor and instead aim to identify organisms after a pre-determined deployment period (e.g. Cohen et al., 2005; Arenas et al., 2006; Floerl et al., 2012; Mineur et al., 2012; Bishop et al., 2015). Succession can play a key role in the formation of fouling communities (Berntsson and Jonsson, 2003; Langhamer, Wilhelmsson and Engström, 2009; South, Floerl and Jeffs, 2019), and by sampling regularly through the year we can develop a much more detailed picture not only of the organisms that are present within port communities but also how certain species can shape the communities. This was particularly evident from within the Port of Swansea where I was able to highlight the role that solitary ascidians, particularly Ascidiella scabra, may play in the development of a species rich community (Chapter 1). A more regular sampling strategy also increases the likelihood of identifying NNS. A number of NNS reported within this thesis would indeed not have been recorded had samples been collected only once or even twice through the peak colonisation season.

The MMS was designed with a more applied purpose, focusing on the management of NNS colonisation based on the materials present within port environments. NNS have regularly been reported to colonise a wide range of artificial structures (Glasby et al., 2007; Tyrrell and Byers, 2007; Mineur et al., 2012; O'Shaughnessy et al., 2020), with some studies focussing on identifying differences in NNS colonisation between natural and artificial substrates (Glasby et al., 2007; Tyrrell and Byers, 2007; O'Shaughnessy et al., 2020). 
Rapid assessment surveys (RAS) achieve a similar outcome through the surveying of multiple substrate and habitat types (e.g. Arenas et al., 2006; Collin et al., 2015; Bishop et al., 2015; Rohde et al., 2017). The use of RAS within ports is rarely a viable option due in a large part to the difficulty in accessing and working with submerged port infrastructure (General Introduction, Chapters 1 and 3; HELCOM, 2013). The MMS offers a compromise between RAS and traditional settlement surveys. The colonisation of different material types can be monitored, which can offer an added level of detail to NNS management. I reported in Chapters 1 and 3 that material type was a significant factor in determining the observed community. I focussed more on NNS colonisation by material type in Chapter 3 and determined that whilst whole community composition was influenced by material type, NNS were observed to consistently colonise a wide range of materials.

Each of the broad survey designs that I used in completing this research are not without their flaws when it comes to potentially missing NNS and in accurately describing the 'natural' port fouling communities. A number of factors may come into play which influence the observed communities and NNS found colonising the survey materials, including water depth effects and the probability of detecting rare species. Depth is known to influence the colonisation of benthic fauna, even over relatively small differences in depth that were experienced within this research (Hurlbut, 1991; Head, Davenport and Thomason, 2004; Kazmi et al., 2020). Both survey designs may have been influenced by depth effects on colonisation. The SSS were all deployed as accurately as possible to the same depth, although water levels did vary within each port over the course of deployment. This may mean that species went undetected which were colonising port walls at a different depth, either shallower or deeper than the deployment depth of the SSS frames. Materials deployed as part of the MMS were constructed in a set sequence which extended over approximately $2 \mathrm{~m}$. As such the colonisation of materials situated near the top and bottom of each material chain may have been influenced by depth as well as the material type. Surveys could be adapted to take into account depth as a factor, for example by deploying settlement tiles at different depths (Dafforn, Johnston and Glasby, 2009; Lezzi and Giangrande, 2018b) for the SSS and by randomising the sequence of materials within the MMS. 
Practical aspects must though be considered, namely not overweighting the SSS with multiple frames and ensuring the weight distribution in the MMS would prevent materials from drifting away from port walls which would cause problems for port operations.

Settlement tiles are a widely used and accepted methodology for the detection of NNS and description of wider communities (Glasby, 2000; Glasby and Connell, 2001; Floerl et al., 2012; Bangor University, 2015; Collin, Tweddle and Shucksmith, 2015). Whilst research has been published to validate the use of settlement tiles in identifying NNS (Marraffini et al., 2017), there has been little consideration within surveys for the probability of detection of rare species, a conclusion I drew in Chapter 4. Understanding how many settlement tiles are required to detect rare NNS is an important factor beyond the proof of concept that settlement tiles are suitable to detect NNS (Ma, 2020). Research has been conducted previously to determine the minimum number of settlement tiles necessary to identify rare species, including rare NNS (Ma, 2020). This research has, however, focussed on much larger areas than port environments and along open coastline, as opposed to enclosed environments within ports. Ma (2020) for example was able to determine the minimum number of settlement tiles necessary to identify a range of rare species along a section of Canadian coastline and was essentially working with the assumption that the $n$ value is unlimited. Ports do not suit this same assumption; there is always an upper limit to the $n$ value, and it will almost certainly not be as high as statistically desirable. As ports are relatively small and enclosed areas with numerous hazards, it is possible to determine the maximum number of deployment sites which satisfy both the science and the safety aspects. Even when employing survey designs which allow for multiple settlement tiles at each deployment site, for example the frames used here in the SSS or by deploying tiles at different depths (e.g. Lezzi and Giangrande, 2018), there are practical considerations which would likely limit the number of tiles which can be deployed at each site. I suggest a pragmatic approach to surveying within ports is to deploy a minimum of five replicates, or as many settlement tiles as is reasonably possible given the practical working constraints of the environment, which is what was done throughout this research. Coupled with the notion of probability of detection and sample size is the issue of losing survey materials. 
I encountered this problem on a number of occasions throughout my research, as the variable $n$ values reported in Chapters 2 and 3 demonstrated. When the sample size is already low in comparison to ideal survey designs it can be problematic to then lose samples during the period of deployment. Many of my samples happened to be lost through freak accidents, including damage caused by a lock gate suspended out of the water for removal, or the loss of a whole MMS survey by the suction of a French warship bow thruster. Even the best practices to maintain surveys during deployment could not overcome these unfortunate incidents which, again, are part and parcel of working within active ports. It does however reinforce the idea that as many deployment sites should be used as is reasonably possible. Of course, a loss of replicates does reduce the power of statistics, effect sizes, potential for Type II errors and therefore ability to make accurate conclusions based on observations which was the case frequently throughout this thesis.

The development and overall successful application of these two survey methods within active ports has, in my opinion, made a significant step forward towards establishing a standardised methodology for providing a baseline knowledge of NNS and identifying future species introductions within UK ports. Associated British Ports (ABP) have seen first-hand how the knowledge gained from these methods can contribute towards biosecurity measures and help them to satisfy the legislative requirements on NNS monitoring. It is also hoped that the development of a NNS field identification guide tailored for use by ABP employees (Appendix IV) will enable an increase in passive monitoring of NNS within the studied ports and further afield, which may be effective in documenting the spread and introduction of NNS.

We are beginning to see elsewhere in Europe how a well-coordinated survey programme can yield large amounts of data covering a range of different biological disciplines and, ultimately, improve our understanding of port environments and how best to manage the introduction of NNS (Di Poi et al., 2019; Kraus et al., 2019; Mandić et al., 2019; Petrocelli et al., 2019; Travizi et al., 2019; Vidjak et al., 2019). For example, Travizi et al. (2019) were able to survey the macrozoobenthos of five Adriatic Sea ports and identified five NNS within the region, applying the use of SCUBA divers to collect hand cores as well as occasional box coring from a vessel along pre-determined survey transectc. 
Whilst the ratio of non-native to native species was low within the studied ports, the data were used to build an understanding of the anthopogenic pressures regarding NNS introductions within the region as well as providing a substantial baseline knowledge of port communities which will allow for future NNS introductions to be catalogued. Work such as this and the insights presented within this thesis is a clear indication that valuable research can, and should, be conducted within ports which can be of importance for stakeholders and policy makers as much as the wider research community.

\section{III.I.I. Future direction of port-based surveys}

I expect the future of port-based surveys and NNS monitoring to change over time, with a shift toward applying more molecular techniques being the most likely and most beneficial outcome. The use of environmental DNA (eDNA) is becoming increasingly popular as a tool in screening for NNS (e.g. Goldberg et al., 2016; Tricarico et al., 2017; Borrell et al., 2017; Yee et al., 2019), with some techniques also offering insight into relative abundances of different species as well as simply informing as to the presence/ absence of a species (Skovhus et al., 2004; Lee et al., 2017; Robinson et al., 2018). I dedicated a period of time early in this research project to evaluate different DNA based approaches with a view to potentially applying an element of molecular screening in the context of this research. Metabarcoding of eDNA, which can be isolated from easily collectable water samples, seemed an ideal option for application in port environments where the deployment of survey materials can be problematic (Borrell et al., 2017). I encountered two primary shortcomings of using molecular tools, although it should be noted this is specifically within the context of my research focus, which may not apply in other areas of research. Firstly, the cost of metabarcoding could not be accommodated within my research grant. Research has, however, been published indicating that metabarcoding of eDNA samples from port environments is a cheaper alternative to conventional settlement surveys (Borrell et al., 2017). Borrell et al. (2017) drew this conclusion based on the fact that the time and expertise required to conduct visual taxonomic surveys brings costs up. In my situation, whereby no such costs were incurred, metabarcoding remained by far the more expensive option. 
Alternative targeted DNA approaches exist which can dramatically cut costs, such as the multiplex method as used by Robinson et al. (2018), and could be used to screen for targeted species such as those on national watch lists (e.g. Welsh Government, 2017). This technique is however not without its flaws, which pertain more to the second shortcoming than to cost.

The second and more widely relevant shortcoming was the fact that not all species have undergone whole genome sequencing. If the aim of using DNA metabarcoding is to screen for NNS, organisms must be identified to species level. For example, identification of a mussel to the genus level of Mytilus would do little to inform as to the native or non-native classification and would instead leave a number of organisms classed as cryptogenic. Targeted DNA approaches can also encounter similar problems if target species have not been sequenced, which is something I found even for common species like Ficopomatus enigmaticus. This problem is, however, rapidly becoming less relevant with the continued efforts to sequence more and more species. A targeted molecular marker for F. enigmaticus has indeed been identified since I encountered the problem (Muñoz-Colmenero et al., 2018).

Whilst the use of molecular techniques is likely to increase in the years to come it remains, from a management perspective, that the use of settlement surveys as used within this thesis can provide critical information to stakeholders, such as community succession or material preferences, which would otherwise be overlooked in molecular screening programmes. A combined approach, whereby molecular methods are used in place of visual taxonomy to identify colonising species from field surveys, may be useful in cutting time and costs (Borrell et al., 2017). This may also be effective in identifying certain species when identification to species level is not possible morphologically, for example if the specimen is not intact (e.g. Syllidae sp., Chapter 1) or if there are gaps in identification keys or guides (e.g. Xanthidae sp., Chapter 2).

Underwater video methodologies are another tool which could be potentially valuable in the monitoring of NNS within port environments. I explored the use of capturing video footage of port walls in Chapter 4, however this was intended only to provide an element of 'ground-truthing' to the settlement surveys which provided all of the quantitative data for the Chapter. 
I found that whilst a lot of community detail was not captured from the video footage, several distinctive species could be identified, including the NNS Bugulina stolonifera. It is worth noting though that the identification of $B$. stolonifera must first be achieved by the examination of a specimen, or through DNA methods, as it would not be morphologically distinctive from other bryozoan species (e.g. $B$. simplex or Tricellaria inopinata) in video footage. Settlement studies can provide information on the coverage of certain species at specific point sampling locations, but they cannot necessarily be used to provide detailed species distribution information. For morphologically distinctive NNS such as Ficopomatus enigmaticus (identified from Newport, Cardiff and Barry) underwater video techniques, including the use of remotely operated vehicles (ROVs), could be used in rapid screening programmes, for species distribution mapping, and to inspect vessel hulls as part of biosecurity management procedures. The use of ROVs has in the past been restricted, by the size and cost of the equipment, to broad-scale and meso-scale surveys (Bo et al., 2014; Cánovas-Molina et al., 2016), however recent advances in the field have applied the use of mini-ROVs which can be used for much smaller scale surveys (Buscher et al., 2020).

\section{III.II. Management of non-native species}

The management of non-native species (NNS) has been a recurring theme throughout this thesis as it is one of the fundamental reasons for conducting portbased monitoring of NNS. Approaches to NNS management are largely dependent on the stage of invasion at which a species is identified, whereby the management options available at an earlier stage of invasion may be more focussed on eradication and containment, whilst impact mitigation, for example through controlled removal from structures to limit biofouling effects, is the only real option once a species becomes established within an environment (Blackburn et al., 2011). Attempts have been made more recently to speed up decision making processes, enabling effective management strategies to be implemented more quickly and thus provide a greater chance of eradiation or slowing the invasion process (Giakoumi et al., 2019). 
Management of aquatic NNS is, however, slightly more challenging compared to terrestrial NNS management, with eradication being a rarely successful management option (Sambrook et al., 2014; Cook et al., 2015; Booy et al., 2017). The one common theme among all management schemes is that the earlier a species is detected, the more effective the management strategy will be (Blackburn et al., 2011; Giakoumi et al., 2019).

I made the point back in the General Introduction that a lack of baseline data can cause difficulties when monitoring NNS. A lack of baseline data from any environment, not just port environments, makes it impossible to know whether a detected NNS is a newly arrived species or whether it has existed in the environment for a number of years or even decades. As such, management of that species can become difficult as we do not know which stage of invasion the species is at. The documentation of both native and non-native species presented in Chapters 1-4 has developed a now substantial pool of baseline data from the five studied ports, which future monitoring efforts can refer to and use to inform management strategies.

Despite the fact that NNS have been associated with potentially high-profile impacts such as the loss of biodiversity and species extinctions for many years (Bax et al., 2003; Molnar et al., 2008; McGeoch et al., 2010), legislation making direct reference to the management and control of NNS has only been developed relatively recently within Europe (reviewed in Boon et al., 2020). There is also evidence to suggest the interpretation and/ or understanding of the current legislation by different European countries is highly varied, even down to the definition of a non-native species (Boon, Clarke and Copp, 2020). There is clearly a need for more direct guidance on the topic of NNS, in support of achieving the legislative goals. An increased understanding of the state of NNS within the UK would contribute toward producing more well-informed policy and guidelines. If the legislative goal of reducing the spread and impact of NNS is a genuine objective, research similar to that presented within this thesis must be conducted on a wider scale and ideally in all UK ports.

Locally, research presented within this thesis will be used by Associated British Ports (ABP) to enhance current management strategies and to satisfy the legislative demands on NNS monitoring. 
The stark differences in the communities observed between the five studied ports (Chapters 2 and 3) indicates that management strategies must be specifically tailored for each individual port. Each port has different aspects of NNS management to consider, based both on the observed communities and the risk of further introductions, which may be associated with the environmental conditions (Chapter 2). Species specific management strategies have been widely used to achieve a range of management objectives, including eradication and containment (Anderson, 2005; Sambrook et al., 2014; Booy et al., 2017). Eradication is however, as mentioned previously, a rarely successful management option in aquatic environments (Sambrook et al., 2014; Cook et al., 2015; Booy et al., 2017). A species specific approach targeting containment could be applied within the ports studied here, with abundant species such as Amphibalanus improvisus, Ficopomatus enigmaticus and Bugulina stolonifera being primary targets as these species are commonly associated with hull fouling and therefore secondary spread within a region (Ruiz et al., 2000; Gollasch, 2002; Davidson et al., 2010; Porter et al., 2015; Wrange et al., 2016). The fact that NNS were found to consistently colonise a range of different material types (Chapters 1 and 3) removes the potential for a habitat type management focus, whereby certain port structures could be the focus of management efforts. There have been numerous research studies published indicating an increased colonisation of artificial structures by NNS (Glasby et al., 2007; Tyrrell and Byers, 2007; Mineur et al., 2012; Leclerc et al., 2019), which would suggest a habitat type management strategy may be successful. However, ports can often be unique in the sense that all the habitats are artificial, and we are therefore looking for differences in NNS colonisation between different artificial substrates. No such significant differences were identified within my research (Chapters 1 and 3). Instead, management strategies must focus on minimising the amount of surplus material within each port, including surface litter which may act as an additional vector for secondary dispersal within the region (Rech, Borrell and García-Vazquez, 2016; Miralles et al., 2018; Rech, Borrell Pichs and García-Vazquez, 2018).

Long-term management options may address a wider objective of promoting increased biodiversity. More diverse communities are thought to be more resilient to environmental stressors, as well as increasing productivity and ecosystem services (Hooper et al., 2005; Duffy, 2009). 
Research into the use of 'blue-green infrastructure', whereby coastal marine infrastructure is modified to promote more diverse fouling communities, has been promising and can successfully satisfy both the engineering demands as well as support diverse communities (Perkol-Finkel et al., 2012; Sella and Perkol-Finkel, 2015; Evans et al., 2019). There is also promising evidence to suggest that making subtle adaptations to the existing infrastructure can reduce the ratio of NNS within a community, such as altering shape and texture to promote the colonisation of native species and increase competition with NNS (Sella and Perkol-Finkel, 2015). The use of blue-green infrastructure should therefore be considered for longer-term NNS management within ports, be this through adapting future construction of infrastructure or by making adaptations to existing infrastructure.

\section{III.III. Non-native species in the wider fouling community}

Non-native species have been a primary focus through the conception and implementation of this research. I have highlighted throughout this thesis the impacts that NNS can have on native species, habitats, and whole ecosystems. NNS are widely considered to be one of the greatest threats facing the loss of biodiversity worldwide, primarily through causing species extinctions (Sala et al., 2000; Bax et al., 2003; Molnar et al., 2008; McGeoch et al., 2010), and they can also have significant impacts on ecosystem services and the economy (e.g. Lovell and Stone, 2005; Williams et al., 2010; Katsanevakis et al., 2014). Understanding the impacts of NNS requires not only a good understanding of which NNS are present within a region or ecosystem, but also an understanding of the community into which they have been introduced; an often overlooked factor when monitoring exclusively for NNS

Efforts to document and provide detailed synopses of marine NNS within the UK have been valuable resources when conducting this research. Most of these resources focus on documenting either a specific species or a range of species following surveys of a specific location or region (Naylor, 1957, 1965; Willis et al., 2004; Arenas et al., 2006; Bangor University, 2015; Collin et al., 2015; Hurst, 2016), although there are also a small number of detailed synopses outlining all the known UK NNS (Eno, Robin and Sanderson, 1997; Minchin, Cook and Clark, 2013). 
In both of these cases the focus is very much on NNS and there is little reference made to the native species which form the wider fouling community. Potential impacts of individual NNS have been presented in various reviews (Streftaris and Zenetos, 2006; Williams et al., 2010; Katsanevakis et al., 2014) and can be highly varied, from positive to negative, which is often dependent on the environment or community in which they are present. Many NNS also have unknown impacts, with the impacts of half of all UK macrofaunal NNS (36 species) listed as unknown in one review (Minchin, Cook and Clark, 2013). While my research also focused on NNS, I have done this in the context of the wider fouling community. In order to fully understand the impacts of NNS and the role that they play within a community it is important to know how the NNS fit into the wider community. Still, whilst I was able to describe the extent with which NNS are present within each port community, for example the dominance of Amphibalanus improvisus in Newport and Ficopomatus enigmaticus in Cardiff, I did not research the direct impact that these species may be having on their respective communities.

From a research perspective it would certainly be interesting to know how NNS can influence the ecosystem functioning of a port community. Given the documented variation in communities between ports (Chapters 2 and 3) it would be expected that the role NNS play within each port community would also differ. Microcosm experiments are commonly used to quantify ecosystem function (Benton et al., 2007; Clare et al., 2016) and could be applied as an extension to settlement surveys whereby colonised settlement panels are monitored to assess the function of communities which have different ratios of non-native to native species. Increasing our understanding of the role NNS play in communities present within the UK, and the potential impacts, would perhaps go one step further toward influencing policy makers. The need to address evidence gaps beyond economic impacts is indeed a point mentioned in 'The Great Britain Invasive Non-native Species Strategy' (UK Government, 2015). 


\section{III.IV. A note on impacts of the Covid-19 pandemic}

Fortunately, the disruption caused by the Covid-19 pandemic has had only a relatively small impact on my research. There are however a couple of alterations I was forced to make to the project in response to the national lockdown and closure of university facilities. I had been collecting monthly plankton samples from each of the five ports from late summer 2019 and intended on collecting for a full 12 months, encompassing the most important period from around March through to July. As the national lockdown in the UK came into force during March 2020 I was forced to terminate the plankton research and, since I was lacking the key period of the year for plankton abundance, what little data I had to that point did not warrant reporting within this thesis.

A second change to my research came to the survey reported within Chapter 4, whereby I analysed the colonisation of plastic and natural substrates. My plan was to analyse each settlement tile in a laboratory, enabling a more detailed analysis into smaller and cryptic species. Due to the extended closure and restricted access to university facilities through the late summer of 2020 I was forced instead to do a field-based analysis of macrofauna. This no doubt limited the number of species identified but was the only option that allowed me to adhere to the government and university Covid-19 guidelines. 


\section{CONCLUSIONS}

- Port environments can, and should, be studied with respect to monitoring non-native species within the UK. This is an achievable goal when using tailored survey methods and collaborating with port operators.

- Two novel survey methods have been designed, created, and successfully deployed within active ports which have been shown to provide important insights into the formation of port fouling communities whilst detecting nonnative species.

- 13 non-native species were identified across the five studied ports. 12 of these species are considered 'established' within the UK. Nine of the observed nonnative species may be classed as sessile benthic filter feeders, which is a functional group commonly associated with the hull fouling vector of species dispersal.

- Communities documented within each isolated port were found to be significantly different to all other ports. Salinity was the primary variable associated with this variation, although a large portion of the variability (ca. $60 \%$ ) remained unexplained. This implies the need to monitor all ports, without making assumptions of port communities based on location or environmental conditions.

- Whole fouling communities were observed to differ between substrate type when data were pooled across all five studied ports, however this was not the case for all ports when analysed independently. Non-native species were found to readily colonise different material types commonly found within port environments, and this was the case across ports with different environmental conditions. A habitat-based approach to management of nonnative species would therefore not be appropriate in port environments. 
- Aspects of community succession were evident in each of the five studied ports. However, the feature of succession, be it in the number of species, total abundance or percentage cover, varied between ports. This further highlights the complexity observed in the formation of fouling communities. Surveying for succession did though increase the likelihood of identifying species which were not consistently recorded in samples, including several non-native species which may otherwise have been absent from the surveys.

- The use of plastic as a substrate in settlement studies was validated as a survey tool. Results indicated that more non-native species were identified on average colonising plastic than a natural substrate. However, data were limited based on the sampling design and loss or replicate samples during the survey.

- Findings from this research have been relayed to Associated British Ports (ABP) and will be incorporated into biosecurity plans. The surveys deployed will continue to be used within the five surveyed ports with hopes for further deployment across the remaining $21 \mathrm{ABP}$ ports and a view toward establishing an active non-native species monitoring programme. This will be supplemented by passive monitoring with the aid of the field guide presented within this Appendix IV. 


\section{APPENDIX I: SUPPLEMENTARY TABLES}


Supplementary Table S1 (Chapter 1). Total abundance/ area covered $\left(\mathrm{cm}^{2}\right)$ of species within each survey month, identified as part of the Successional Settlement Survey. Area covered is denoted by a ‘*`, all other numbers are abundance values. Species are grouped within Phylum. NNS denoted by a ' $\dagger$.

\begin{tabular}{|c|c|c|c|c|c|c|c|c|c|c|c|c|c|}
\hline \multirow{2}{*}{ Species } & \multicolumn{6}{|c|}{ Month } & \multirow{2}{*}{ Species } & \multicolumn{6}{|c|}{ Month } \\
\hline & Jun & $\overline{\mathrm{Jul}}$ & Aug & Sep & Oct & Nov & & Jun & $\overline{\text { Jul }}$ & Aug & Sep & Oct & Nov \\
\hline Porifera & & & & & & & Echinodermata & & & & & & \\
\hline Grantia compressa & - & - & - & 3 & 5 & 1 & Amphipholis squamata & - & 1 & - & 1 & - & 1 \\
\hline Cnidaria & & & & & & & Arthropoda & & & & & & \\
\hline Aurelia aurita (polyps) & - & - & - & 18 & 111 & 150 & Austrominius modestus ${ }^{\dagger}$ & - & 3 & - & 5 & 14 & 19 \\
\hline Obelia longissima ${ }^{*}$ & - & - & - & - & 1 & - & Balanus crenatus & - & - & 1 & - & - & 3 \\
\hline Platyhelminthes & & & & & & & ${\text { Brachynotus sexdentatus }{ }^{\dagger}}^{\dagger}$ & - & - & 1 & - & - & - \\
\hline Prostheceraeus vittatus & - & 2 & 3 & - & - & - & Caprella mutica ${ }^{\dagger}$ & - & 2 & 19 & 14 & 9 & 10 \\
\hline Annelida & & & & & & & Carcinus maenas & - & - & - & 1 & 3 & - \\
\hline Harmothoe imbricata & - & - & 7 & - & - & - & Dexamine spinosa & - & - & 1 & 1 & 1 & 3 \\
\hline Lepidonotus squamatus & - & - & - & 5 & 7 & 13 & Endeis spinosa & 4 & 2 & 1 & - & 11 & - \\
\hline Nereimyra punctata & - & - & 2 & 7 & 3 & 3 & Inachus phalangium & - & - & - & 1 & 1 & - \\
\hline Platynereis dumerilii & - & - & - & - & - & 1 & Lembos websteri & 14 & 14 & 14 & 25 & 14 & 5 \\
\hline Psamathe fusca & - & 2 & 2 & - & - & - & Macropodia deflexa & - & - & - & 1 & - & - \\
\hline Spirobranchus triqueter & 2 & 2 & 1 & 6 & 11 & 4 & Monocorophium acherusicum ${ }^{\dagger}$ & - & 4 & - & 8 & 4 & - \\
\hline Syllidae spp. & - & - & - & - & - & 3 & Palaemon serratus & - & - & - & - & 2 & - \\
\hline
\end{tabular}




\begin{tabular}{|c|c|c|c|c|c|c|c|c|c|c|c|c|c|}
\hline \multirow{2}{*}{ Species } & \multicolumn{6}{|c|}{ Month } & \multirow{2}{*}{ Species } & \multicolumn{6}{|c|}{ Month } \\
\hline & Jun & Jul & Aug & Sep & Oct & Nov & & Jun & Jul & Aug & Sep & Oct & Nov \\
\hline Sessilia spp. & 8 & - & - & - & - & - & Botryllus schlosseri ${ }^{*}$ & 2.5 & 24 & 9 & 17 & 26 & 2 \\
\hline Mollusca & & & & & & & Ciona intestinalis & - & 50 & 339 & 343 & 359 & 173 \\
\hline Mytilus edulis & 1 & 5 & 19 & 10 & 29 & 9 & Clavelina lepadiformis & - & 15 & 5 & 9 & 12 & 4 \\
\hline Patellidae spp. & - & - & - & - & - & 1 & Diplosoma listerianum ${ }^{*}$ & 1 & 4.5 & 1.5 & 2 & 16 & 130 \\
\hline Testudinalia testudinalis & - & - & - & - & 1 & - & Phlebobranchia spp. & 6 & - & - & - & - & - \\
\hline Bryozoa & & & & & & & Polycarpa pomaria & - & - & - & - & 1 & 1 \\
\hline Bugula neritina ${ }^{\dagger}$ & - & - & - & 3 & - & - & Pomatoschistus microps & - & - & - & - & - & 1 \\
\hline Bugulina stolonifera ${ }^{\dagger}$ & - & 77 & 54 & 87 & 82 & 46 & Styela clava ${ }^{\dagger}$ & - & 1 & - & 2 & 3 & 3 \\
\hline \multicolumn{14}{|l|}{ Chordata } \\
\hline Ascidiella scabra & - & 55 & 237 & 248 & 243 & 154 & & & & & & & \\
\hline
\end{tabular}


Supplementary Table S2 (Chapter 1). Total abundance and area covered $\left(\mathrm{cm}^{2}\right)$ of species identified from materials forming the Mixed Material Survey. Area covered is denoted by '*’, all other values are abundance counts. NNS denoted by a ‘ ${ }^{\prime}$.

\begin{tabular}{|c|c|c|c|c|c|c|c|c|c|c|}
\hline \multirow[b]{2}{*}{ Species } & \multicolumn{10}{|c|}{ Material } \\
\hline & Brick & $\begin{array}{l}\text { Mop } \\
\text { Head }\end{array}$ & $\begin{array}{l}\text { PMMA Tile } \\
\text { (sanded) }\end{array}$ & $\begin{array}{l}\text { PMMA Tile } \\
\text { (unsanded) }\end{array}$ & $\begin{array}{l}\text { Polypropylene } \\
\text { Rope }\end{array}$ & $\begin{array}{l}\text { PVC Pipe } \\
\text { (sanded) }\end{array}$ & $\begin{array}{l}\text { PVC Pipe } \\
\text { (unsanded) }\end{array}$ & $\begin{array}{l}\text { Sisal } \\
\text { Rope }\end{array}$ & Steel & Wood \\
\hline \multicolumn{11}{|l|}{ Porifera } \\
\hline Grantia compressa & 12 & 4 & 9 & 7 & - & 4 & 1 & - & - & 2 \\
\hline \multicolumn{11}{|l|}{ Annelida } \\
\hline Spirobranchus triqueter & - & - & 2 & - & - & - & - & - & - & - \\
\hline \multicolumn{11}{|l|}{ Arthropoda } \\
\hline Austrominius modestus ${ }^{\dagger}$ & 27 & - & 35 & 21 & - & - & - & - & - & - \\
\hline Balanus crenatus & 8 & - & 12 & 9 & - & - & - & - & - & - \\
\hline Brachynotus sexdentatus ${ }^{\dagger}$ & 1 & - & 1 & - & - & - & - & - & - & - \\
\hline Carcinus maenas & - & 1 & - & - & - & - & - & - & - & - \\
\hline Macropodia rostrata & - & 1 & - & - & - & - & - & - & - & - \\
\hline Palaemon serratus & - & - & 2 & - & - & - & - & - & - & - \\
\hline \multicolumn{11}{|l|}{ Bryozoa } \\
\hline Bugula neritina ${ }^{\dagger}$ & - & - & - & 2 & - & - & - & - & - & - \\
\hline Bugulina stolonifera ${ }^{\dagger}$ & 4 & 18 & 57 & 65 & 9 & 8 & 11 & - & 22 & 28 \\
\hline
\end{tabular}




\begin{tabular}{|c|c|c|c|c|c|c|c|c|c|c|}
\hline \multirow[b]{2}{*}{ Species } & \multicolumn{10}{|c|}{ Material } \\
\hline & Brick & $\begin{array}{l}\text { Mop } \\
\text { Head }\end{array}$ & $\begin{array}{c}\text { PMMA Tile } \\
\text { (sanded) }\end{array}$ & $\begin{array}{l}\text { PMMA Tile } \\
\text { (unsanded) }\end{array}$ & $\begin{array}{c}\text { Polypropylene } \\
\text { Rope }\end{array}$ & $\begin{array}{l}\text { PVC Pipe } \\
\text { (sanded) }\end{array}$ & $\begin{array}{l}\text { PVC Pipe } \\
\text { (unsanded) }\end{array}$ & $\begin{array}{l}\text { Sisal } \\
\text { Rope }\end{array}$ & Steel & Wood \\
\hline \multicolumn{11}{|l|}{ Chordata } \\
\hline Ascidiella scabra & 26 & 32 & 40 & 55 & 18 & 27 & 34 & - & - & 32 \\
\hline Botryllus schlosseri $^{*}$ & - & 6.5 & 1 & 3 & 7 & - & - & - & - & - \\
\hline Ciona intestinalis & 17 & 21 & 84 & 77 & 22 & 20 & 21 & 2 & 19 & 51 \\
\hline Diplosoma listerianum ${ }^{*}$ & 39 & - & - & 2 & 2 & - & - & - & - & - \\
\hline Styella clava ${ }^{\dagger}$ & - & 5 & - & 6 & 7 & 5 & - & - & - & 2 \\
\hline
\end{tabular}


Supplementary Table S3 (Chapter 2). Abundance count or measure of coverage $\left(\mathrm{cm}^{2}\right)$ for each species recorded within each of the five studied ports: Newport, Cardiff, Barry, Port Talbot, Swansea. ${ }^{*}$ denotes species recorded as area covered when abundance counts were not possible. ${ }^{\dagger}$ denotes nonnative species.

\begin{tabular}{|c|c|c|c|c|c|c|c|c|c|c|c|}
\hline Species & $\begin{array}{l}\stackrel{ \pm}{0} \\
\sum_{0}^{0} \\
z \\
z\end{array}$ & 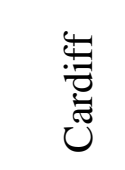 & 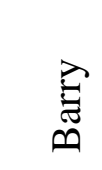 & 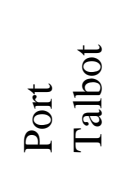 & 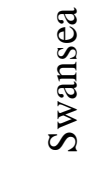 & Species & 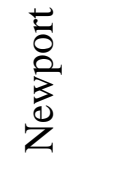 & 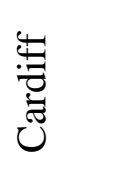 & $\stackrel{\overparen{E}}{\vec{E}}$ & 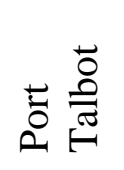 & 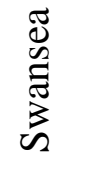 \\
\hline Porifera & & & & & & Harmothoe impar & - & - & 1 & - & - \\
\hline Axinella darmicornis ${ }^{*}$ & - & - & - & - & 3 & Lepidonotus squamatus & - & - & 3 & - & 27 \\
\hline Grantia compressa & - & - & - & - & 36 & Nereimyra punctata & - & - & 2 & - & 6 \\
\hline Stelligera rigidia ${ }^{*}$ & - & - & 10 & - & - & Nereis zonata & - & - & 1 & - & 2 \\
\hline Cnidaria & & & & & & Platynereis dumerilii & 30 & 19 & 5 & - & 3 \\
\hline Cordylophora caspia ${ }^{\dagger^{*}}$ & - & 0.5 & - & 18.5 & - & Spirobranchus lamarcki & - & - & 16 & - & - \\
\hline Diadumene lineata $^{\dagger}$ & - & - & 1 & - & - & Syllis cornuta & - & - & - & - & 2 \\
\hline Laomeda flexulosa ${ }^{*}$ & 1.5 & - & 0.5 & - & 0.5 & Echinodermata & & & & & \\
\hline Obelia longissima ${ }^{*}$ & - & 1 & 0.5 & - & 2.5 & Amphipholis squamata & - & - & - & - & 62 \\
\hline Platyhelminthes & & & & & & Arthropoda & & & & & \\
\hline Prostheceraeus vittatus & - & - & - & - & 1 & Allomelita pellucida & 128 & - & - & - & - \\
\hline Annelida & & & & & & Amphibalanus improvisus ${ }^{\dagger}$ & 10474 & 1117 & - & - & - \\
\hline Exogone naidina & - & - & - & - & 1 & Maera grossimana & - & 1 & - & - & - \\
\hline Ficopomatus enigmaticus ${ }^{\dagger}$ & 7 & 3355 & - & - & - & Microdeutopus gryllotalpa & - & - & 36 & - & 42 \\
\hline
\end{tabular}




\begin{tabular}{|c|c|c|c|c|c|c|c|c|c|c|c|}
\hline Species & 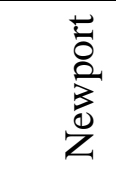 & 节 & $\stackrel{\vec{E}}{\vec{D}}$ & $\stackrel{\varpi}{\stackrel{0}{0}}$ & 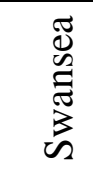 & Species & 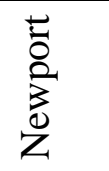 & 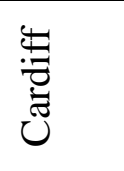 & 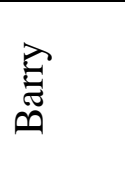 & 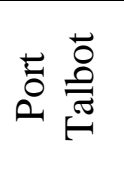 & $\begin{array}{l}\tilde{D} \\
0 \\
0 \\
\tilde{\pi} \\
3 \\
n\end{array}$ \\
\hline Monocorophium acherusicum $^{\dagger}$ & 522 & 3 & 51 & - & 8 & Electra Pilosa $^{*}$ & 152 & 174.5 & - & - & - \\
\hline Monocorophium sextonae ${ }^{\dagger}$ & - & - & 6 & - & - & Fredericella sultana $^{*}$ & - & - & - & 513 & - \\
\hline Xanthidae sp. & - & - & - & - & 1 & Chordata & & & & & \\
\hline Sphaeroma serratum & 3 & 8 & - & - & - & Ascidiella scabra & - & - & 123 & - & 1288 \\
\hline Mollusca & & & & & & Botryllus schlosseri ${ }^{*}$ & - & - & 180.5 & - & 7 \\
\hline Cerastoderma edule & 3 & - & - & - & - & Ciona intestinalis & - & - & 162 & - & 865 \\
\hline Ecrobia ventrosa & - & 20 & - & - & - & Diplosoma listerianum ${ }^{*}$ & - & - & - & - & 13 \\
\hline Mytillus edulis & 1745 & 1189 & 1083 & - & 80 & Molgula socialis & 8 & - & 93 & - & - \\
\hline Mytilopsis leucophaeata ${ }^{\dagger}$ & - & - & - & 4 & - & Styela clava ${ }^{\dagger}$ & - & - & 1 & - & - \\
\hline \multicolumn{12}{|l|}{ Bryozoa } \\
\hline Bugulina stolonifera ${ }^{\dagger}$ & - & - & 1 & - & 240 & & & & & & \\
\hline Cryptosula pallasiana ${ }^{*}$ & 15 & - & - & - & 4.5 & & & & & & \\
\hline
\end{tabular}


Supplementary Table S4 (Chapter 3). Abundance count or measure of coverage $\left(\mathrm{cm}^{2}\right)$ for each species recorded within each of the five studied ports: Newport, Cardiff, Barry, Port Talbot, Swansea. * denotes species recorded as area covered when abundance counts were not possible. ${ }^{\dagger}$ denotes non-native species.

\begin{tabular}{|c|c|c|c|c|c|c|c|c|c|c|c|}
\hline Species & $\begin{array}{l}\overline{0} \\
0 \\
z \\
z \\
z\end{array}$ & 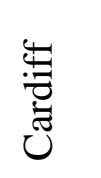 & $\stackrel{\Xi}{E}$ & $\begin{array}{l}\overrightarrow{0} \\
\stackrel{0}{0} \\
\tilde{\sigma}\end{array}$ & 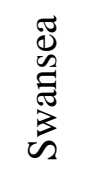 & Species & 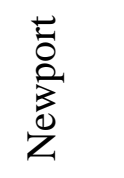 & 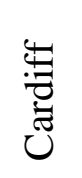 & $\stackrel{\vec{E}}{\oplus}$ & 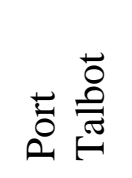 & 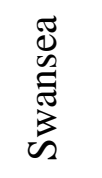 \\
\hline Porifera & & & & & & Macropodia rostrata & - & - & - & - & 1 \\
\hline Axinella darmicornis ${ }^{*}$ & 2 & - & - & - & - & Mollusca & & & & & \\
\hline Grantia compressa & - & - & - & - & 86 & Cerastoderma edule & 1 & - & - & - & - \\
\hline Annelida & & & & & & Mytillus edulis & 4167 & 39 & 1109 & - & - \\
\hline Ficopomatus enigmaticus $^{+}$ & 23 & 562 & 176 & - & - & Bryozoa & & & & & \\
\hline Spirobranchus triqueter & - & - & - & - & 6 & Bugulina stolonifera ${ }^{\dagger}$ & - & - & - & - & 449 \\
\hline Arthropoda & & & & & & Fredericella sultana ${ }^{*}$ & - & - & - & 637.5 & - \\
\hline Amphibalanus improvisus ${ }^{\dagger}$ & 2301 & 407 & - & - & - & Chordata & & & & & \\
\hline Austrominius modestus ${ }^{\dagger}$ & - & - & - & - & 156 & Ascidiella scabra & - & - & 232 & - & 444 \\
\hline Balanus crenatus & - & - & 123 & - & 354 & Botryllus schlosseri ${ }^{*}$ & - & - & 14.5 & - & 22.5 \\
\hline Brachynotus sexdentatus ${ }^{\dagger}$ & - & - & - & - & 1 & Ciona intestinalis & - & - & 232 & - & 365 \\
\hline Caprella mutica ${ }^{\dagger}$ & - & - & 86 & - & - & Diplosoma listerianum * & - & - & - & - & 69 \\
\hline Carcinus maenas & 3 & - & 2 & - & 6 & Molgula socialis & 48 & - & 3 & - & - \\
\hline Gammarus zaddachi & 27 & - & - & - & - & Styela clava ${ }^{\dagger}$ & - & - & 5 & - & 4 \\
\hline
\end{tabular}


Supplementary Table S5 (Chapter 3). Non-native species observed colonising different material types in each of the five studied ports. Port denoted by letter: Newport $=$ N, Cardiff $=$ C, Barry = B, Swansea $=$ S. No non-native species observed from Port Talbot.

\begin{tabular}{|c|c|c|c|c|c|c|c|c|c|c|}
\hline Species & Brick & Mop & $\begin{array}{l}\text { PMMA } \\
\text { (Sanded) }\end{array}$ & $\begin{array}{l}\text { PMMA } \\
\text { (Unsanded) }\end{array}$ & $\begin{array}{l}\text { Polypropylene } \\
\text { Rope }\end{array}$ & $\begin{array}{l}\text { PVC } \\
\text { (Sanded) }\end{array}$ & $\begin{array}{l}\text { PVC } \\
\text { (Unsanded) }\end{array}$ & $\begin{array}{l}\text { Sisal } \\
\text { Rope }\end{array}$ & Steel & Wood \\
\hline $\begin{array}{l}\text { Amphibalanus } \\
\text { improvisus }\end{array}$ & $\mathrm{N}, \mathrm{C}$ & $\mathrm{N}$ & $\mathrm{N}, \mathrm{C}$ & $\mathrm{N}, \mathrm{C}$ & $\mathrm{N}$ & $\mathrm{N}, \mathrm{C}$ & $\mathrm{N}, \mathrm{C}$ & $\mathrm{N}$ & $\mathrm{N}$ & $\mathrm{N}, \mathrm{C}$ \\
\hline $\begin{array}{l}\text { Austrominius } \\
\text { modestus }\end{array}$ & $\mathrm{S}$ & & $\mathrm{S}$ & $\mathrm{S}$ & & & & & & $\mathrm{S}$ \\
\hline $\begin{array}{l}\text { Brachynotus } \\
\text { sexdentatus }\end{array}$ & & $\mathrm{S}$ & & & & & & & & \\
\hline Bugulina stolonifera & $\mathrm{S}$ & $\mathrm{S}$ & $\mathrm{S}$ & $\mathrm{S}$ & $\mathrm{S}$ & $\mathrm{S}$ & $\mathrm{S}$ & $S$ & $\mathrm{~S}$ & $\mathrm{~S}$ \\
\hline Caprella mutica & B & & B & B & B & & & & & B \\
\hline $\begin{array}{l}\text { Ficopomatus } \\
\text { enigmaticus }\end{array}$ & $\mathrm{N}, \mathrm{C}$ & $\mathrm{C}$ & C, B & C, B & $\mathrm{C}$ & $\mathrm{C}$ & $\mathrm{C}$ & $\mathrm{C}$ & $\begin{array}{l}\text { N, C, } \\
\text { B }\end{array}$ & C, B \\
\hline Styela clava & $\mathrm{S}$ & & & & & & $\mathrm{B}$ & & $\mathrm{B}, \mathrm{S}$ & \\
\hline
\end{tabular}




\section{Supplementary Table S6 (Chapter 4).}

Abundance count or measure of coverage $\left(\mathrm{cm}^{2}\right)$ for each species recorded colonising plastic and slate settlement tiles. * denotes species recorded as area covered when abundance counts were not possible. ${ }^{\dagger}$ denotes non-native species.

\begin{tabular}{lrr}
\hline \multirow{2}{*}{ Species } & \multicolumn{2}{c}{ Material } \\
\cline { 2 - 3 } & Plastic & Slate \\
\hline Amphipholis squamata & 2 & 2 \\
Ascidiella scabra & 1960 & 1109 \\
Austrominius modestus ${ }^{+}$ & 77 & 12 \\
Balanus crenatus & 168 & 155 \\
Botryllus schlosseri ${ }^{*}$ & 34 & 22 \\
Bugulina stolonifera ${ }^{\dagger}$ & 686 & 434 \\
Caprella mutica ${ }^{\dagger}$ & 2 & 1 \\
Carcinus maenas & 2 & 0 \\
Ciona intestinalis & 158 & 471 \\
Diplosoma listerianum $*$ & 6.5 & 42 \\
Mytilus edulis & 35 & 26 \\
Platynereis dumerilii & 0 & 2 \\
Spirobranchus triqueter & 14 & 4 \\
Styela clava ${ }^{+}$ & 0 & 1 \\
\hline
\end{tabular}




\section{APPENDIX II: ENVIRONMENTAL VARIABLES (CHAPTER 2)}

This appendix contains plots of environmental time series data collected at the five studied ports, as used in analyses in Chapter 2.

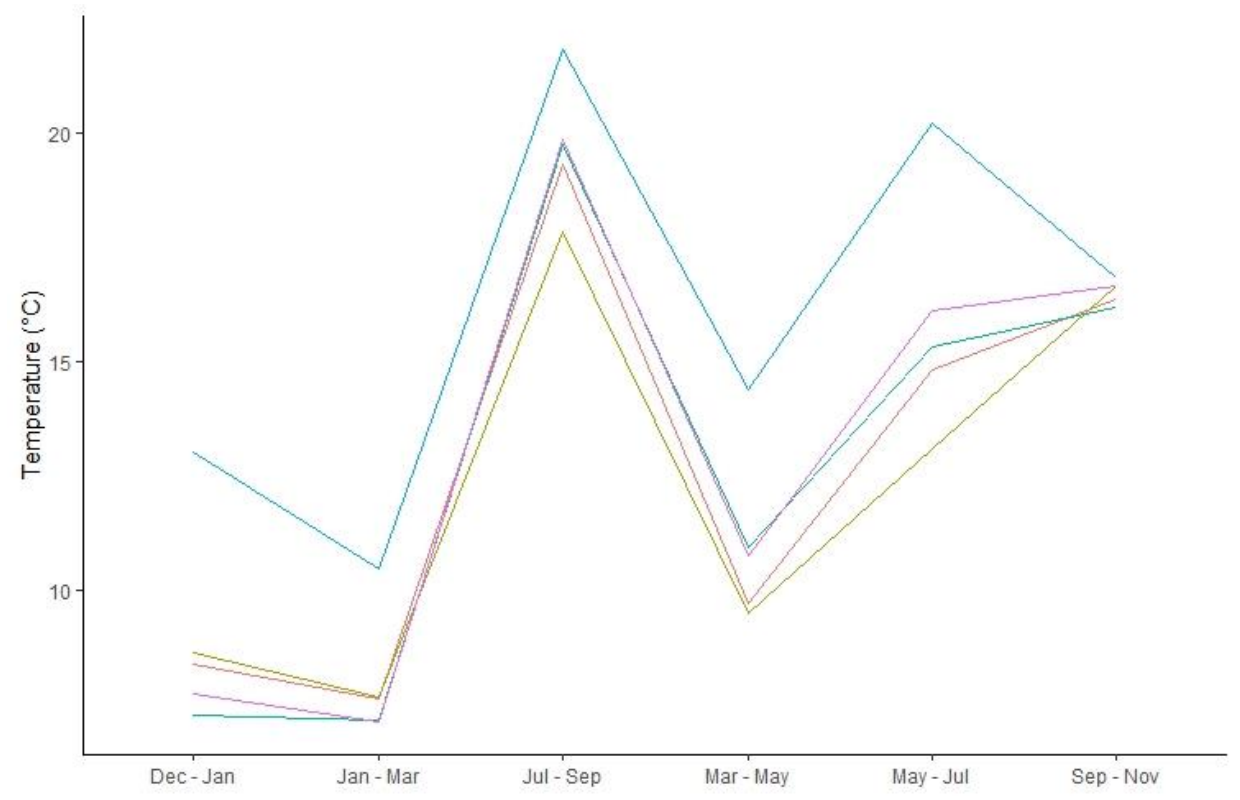

Port

- Barry

- Cardiff

- Newport

- Port Talbot

- Swansea

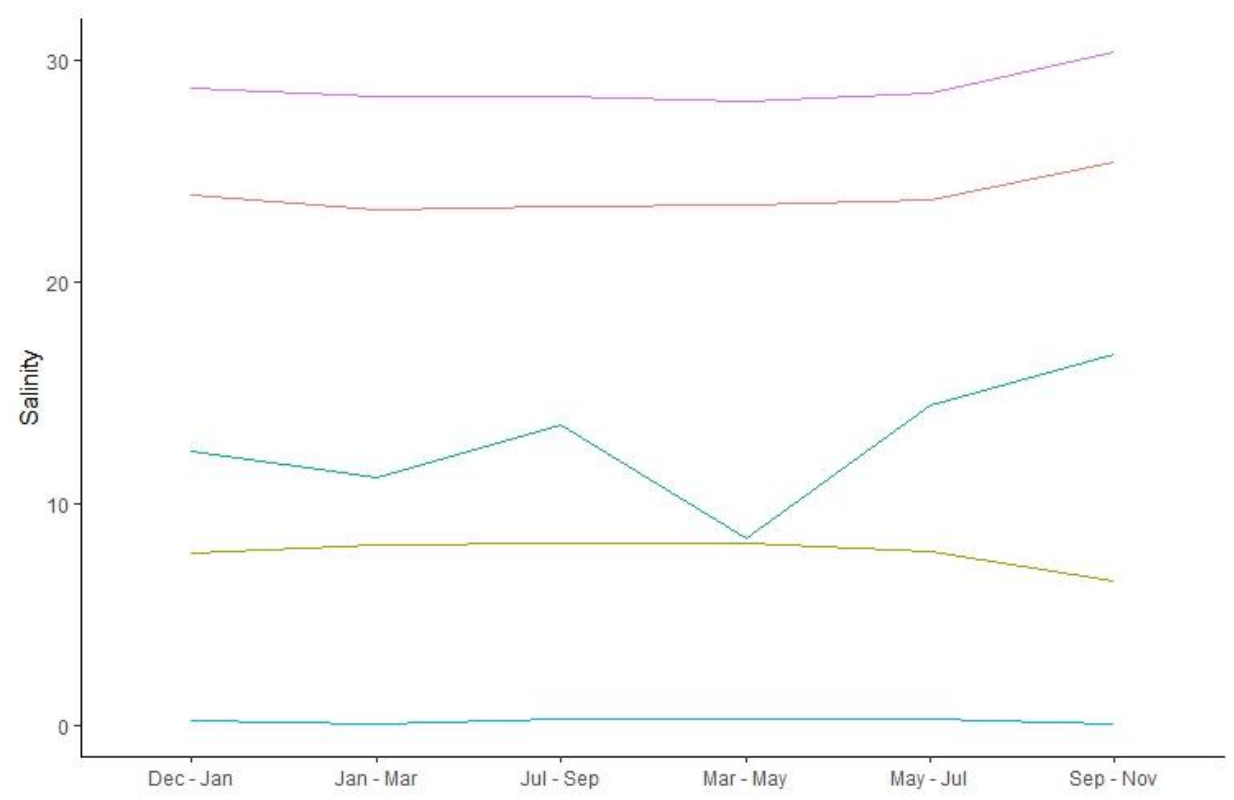

Port

- Barry

- Cardiff

- Newport

- Port Talbot

_ Swansea 

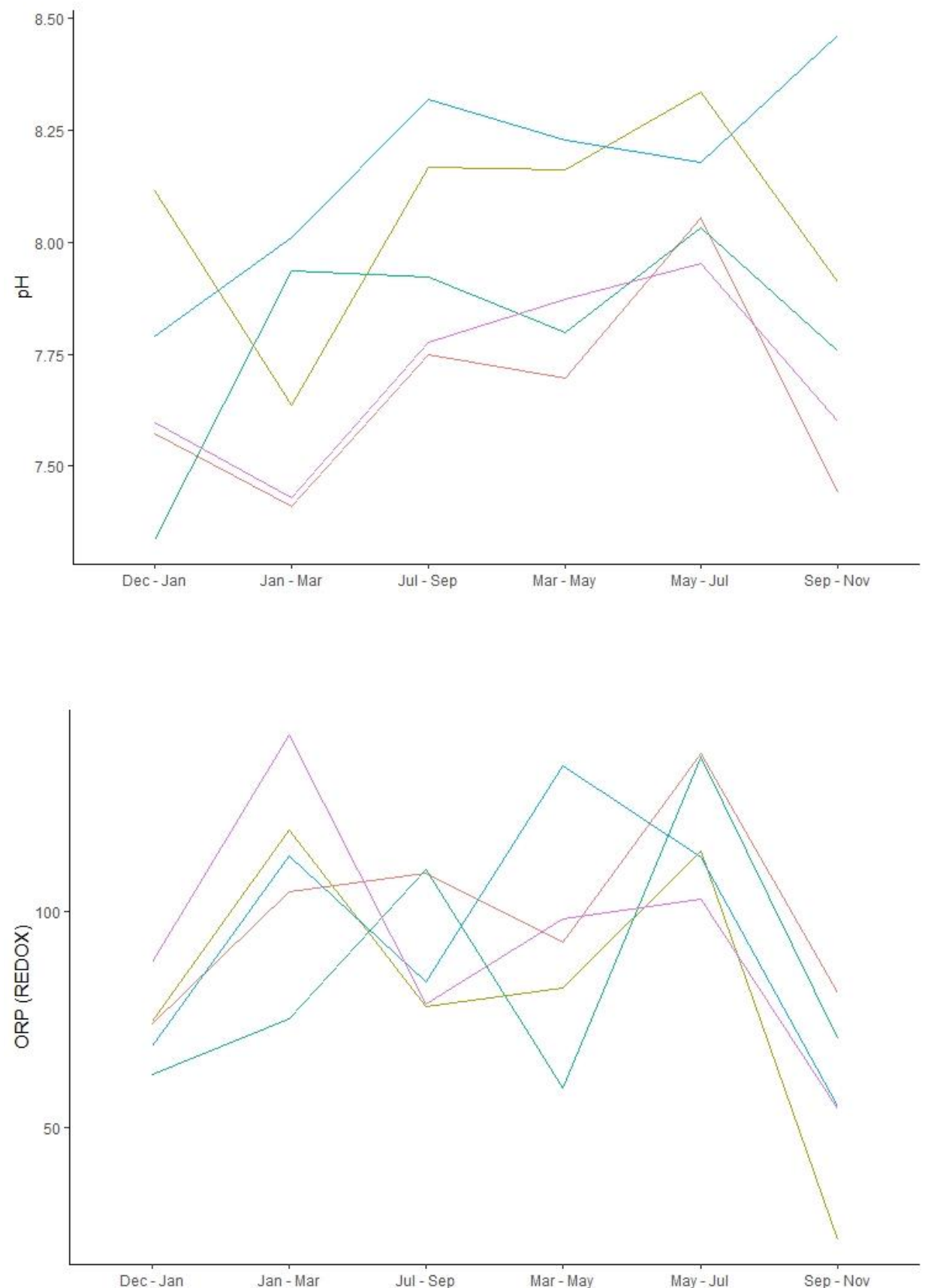


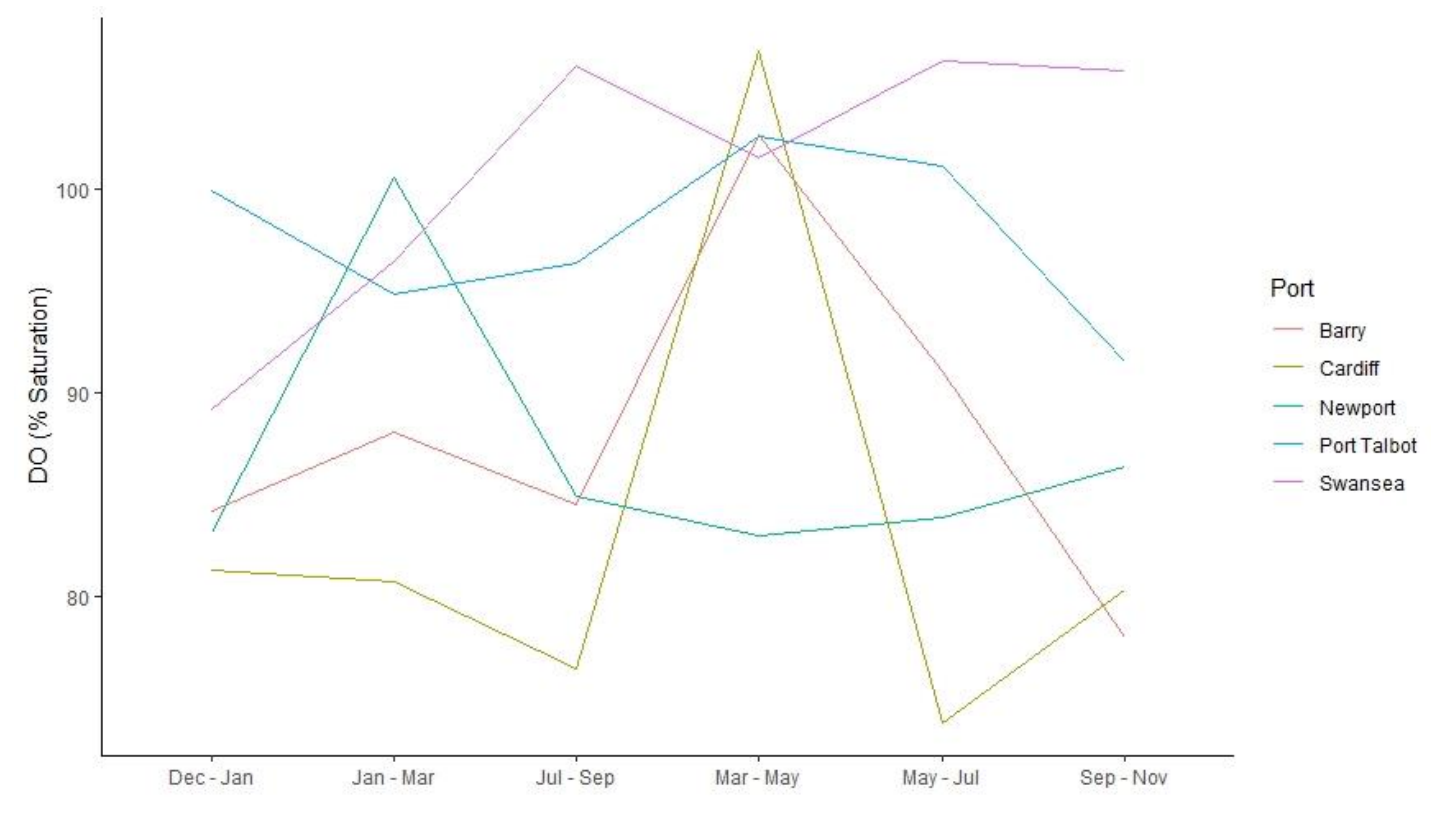




\section{APPENDIX III: SAMPLING PHOTOS}

CHAPTER 1:

SSS: June

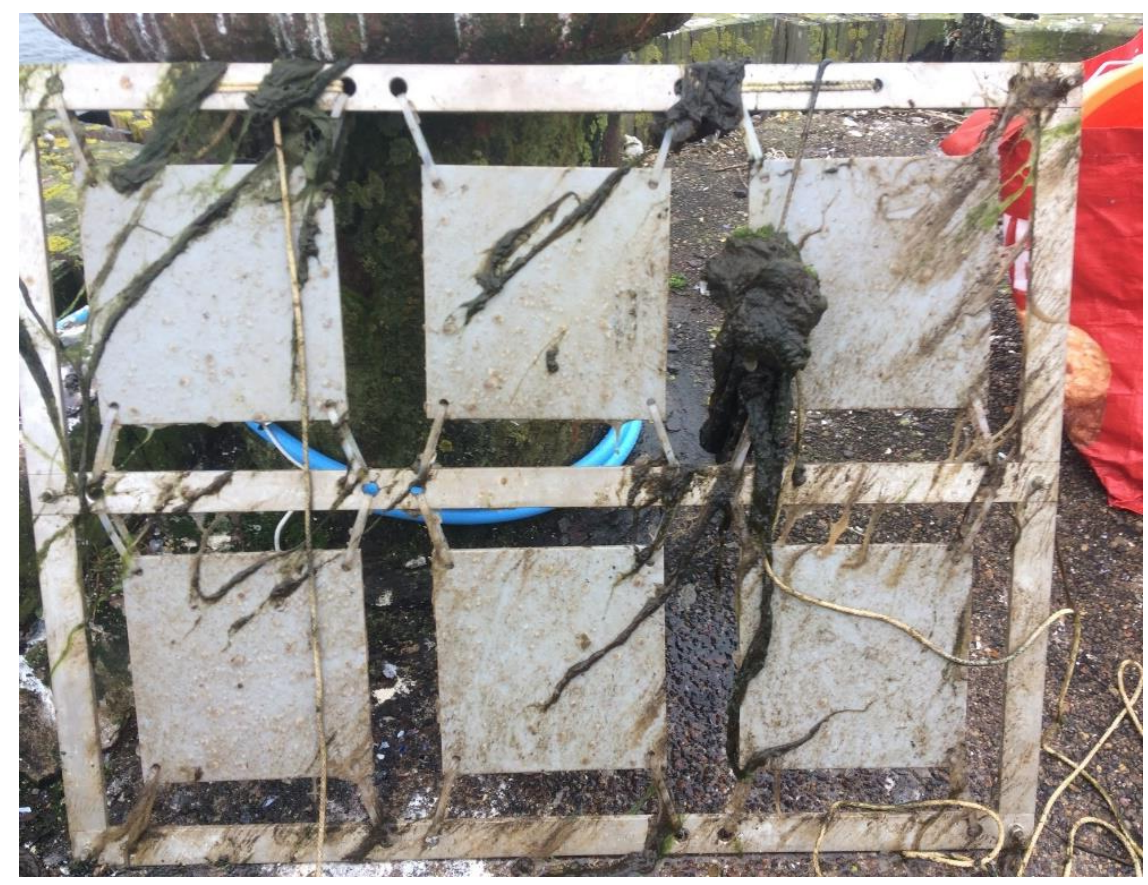

SSS: August

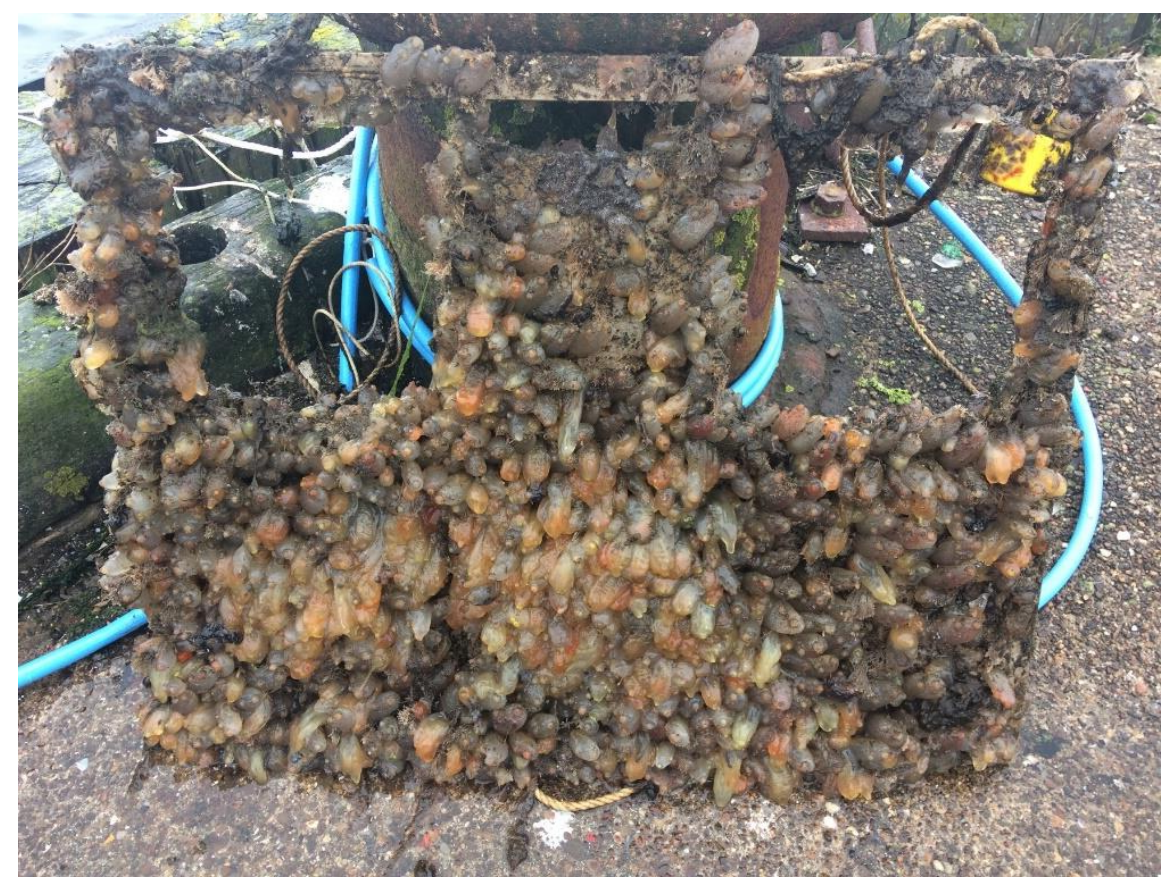


SSS: October

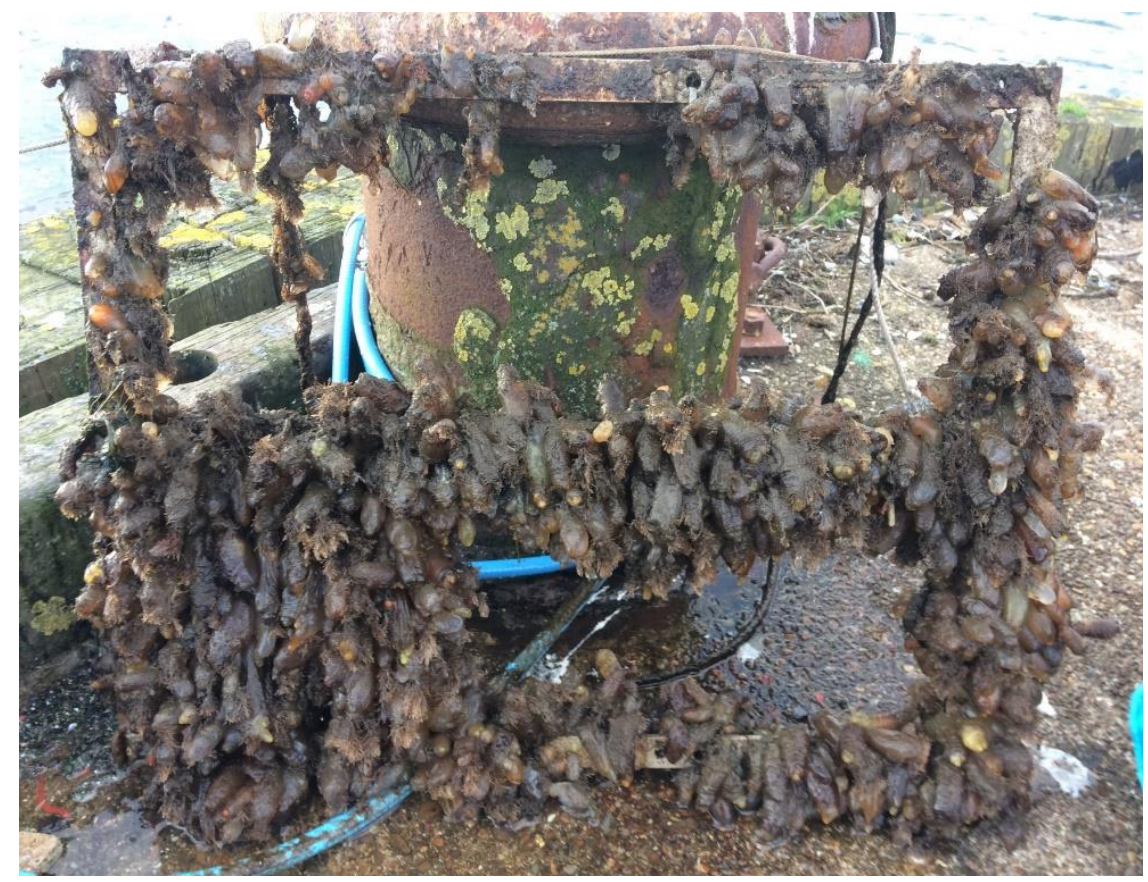

CHAPTER 2:

Newport: Week 6

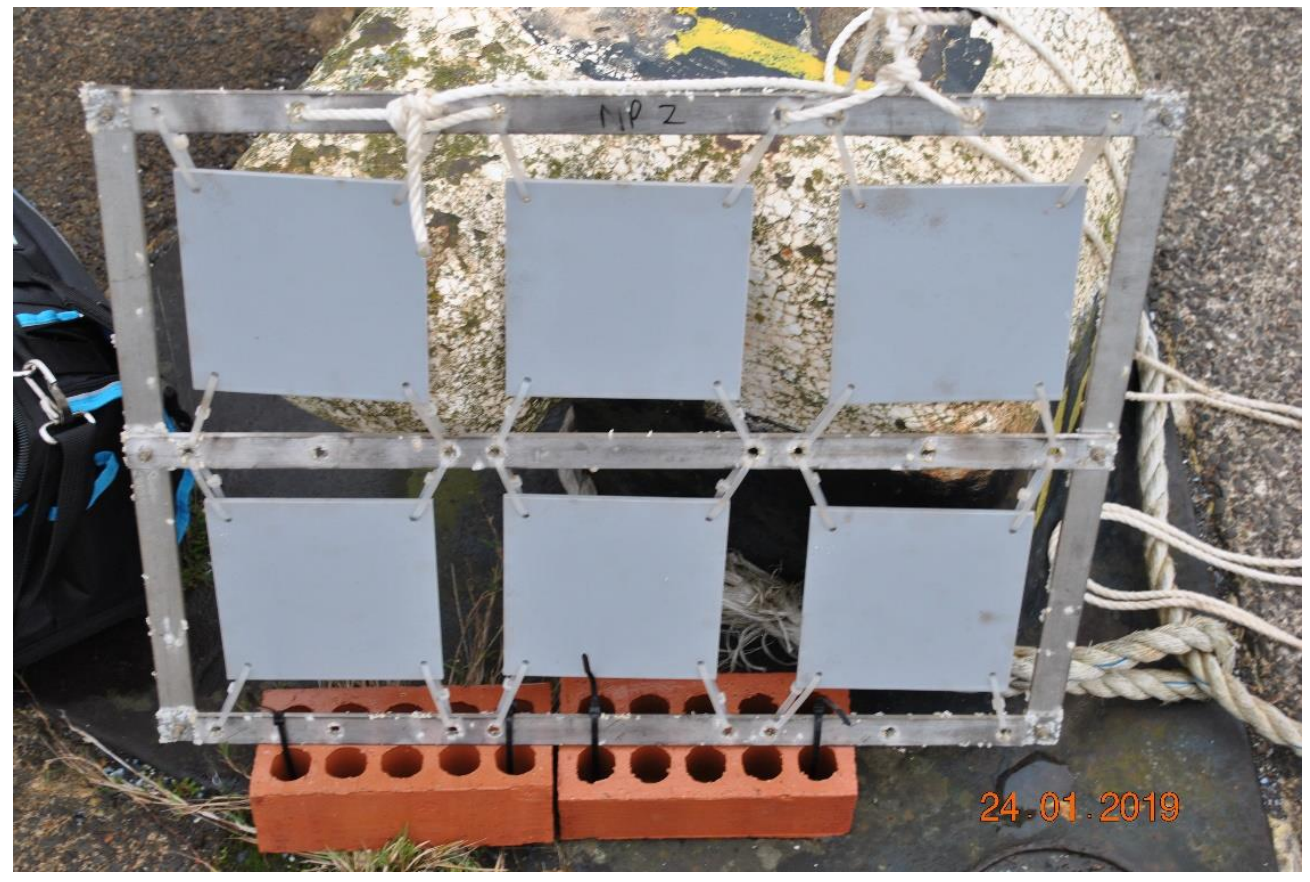


Newport: Week 24

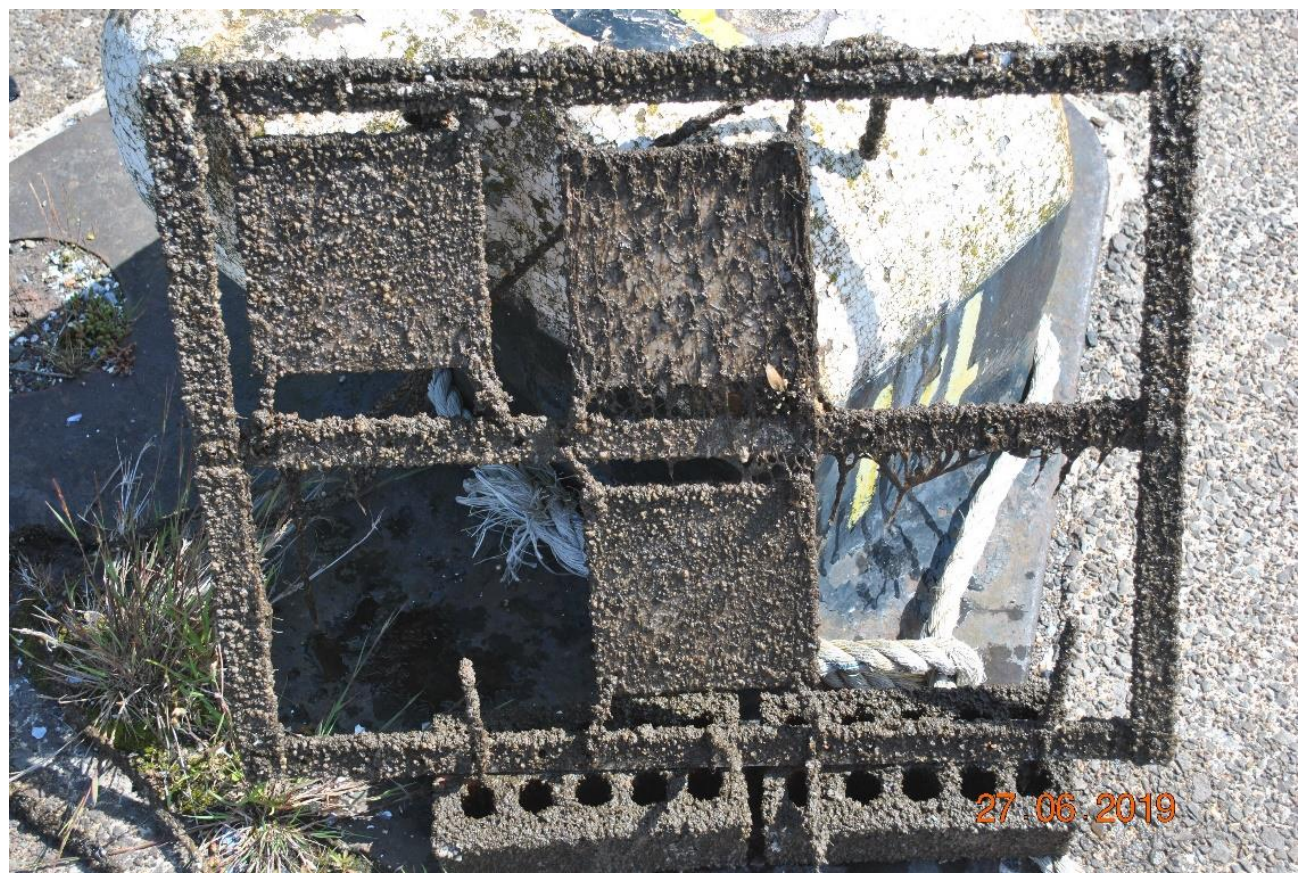

Newport: Week 36

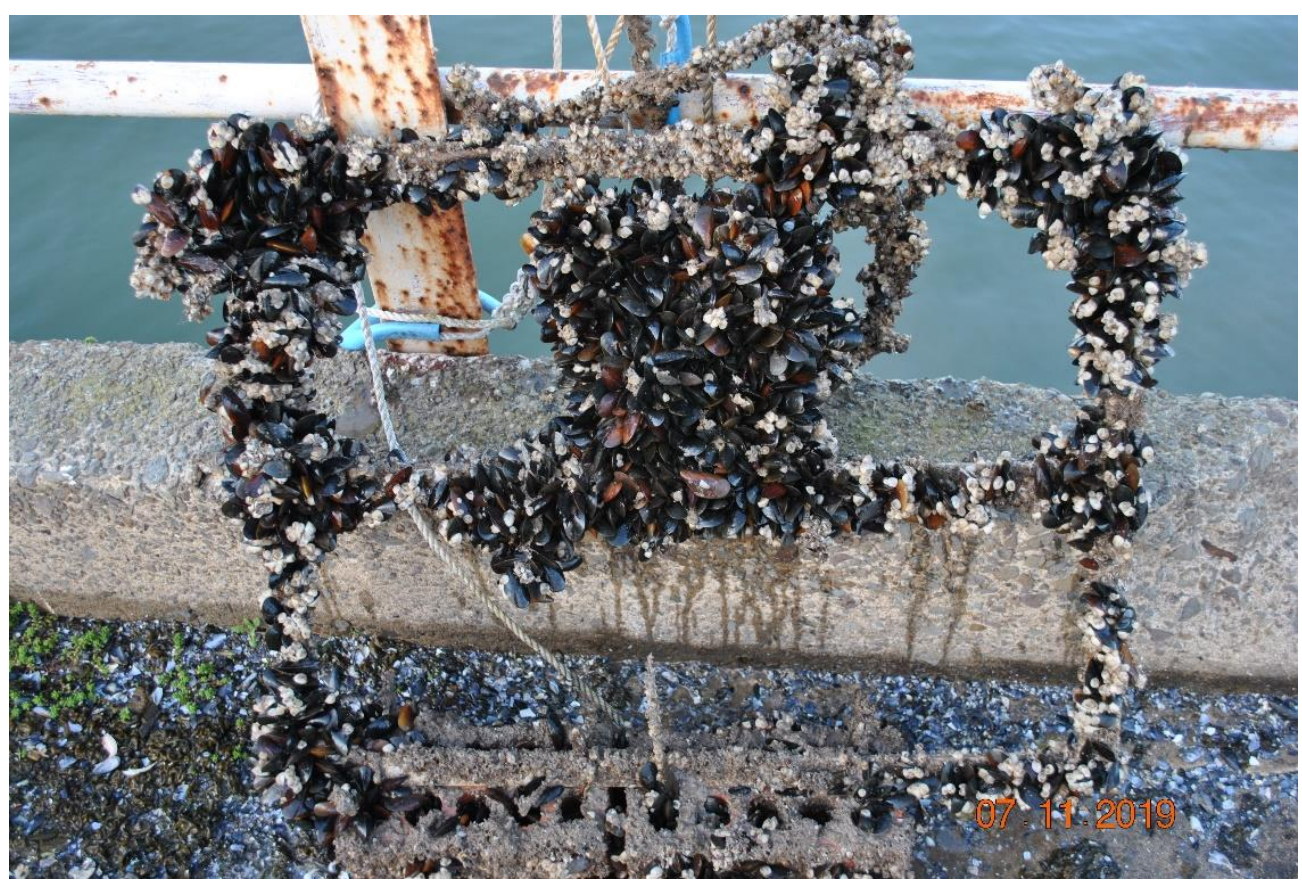


Cardiff: Week 6

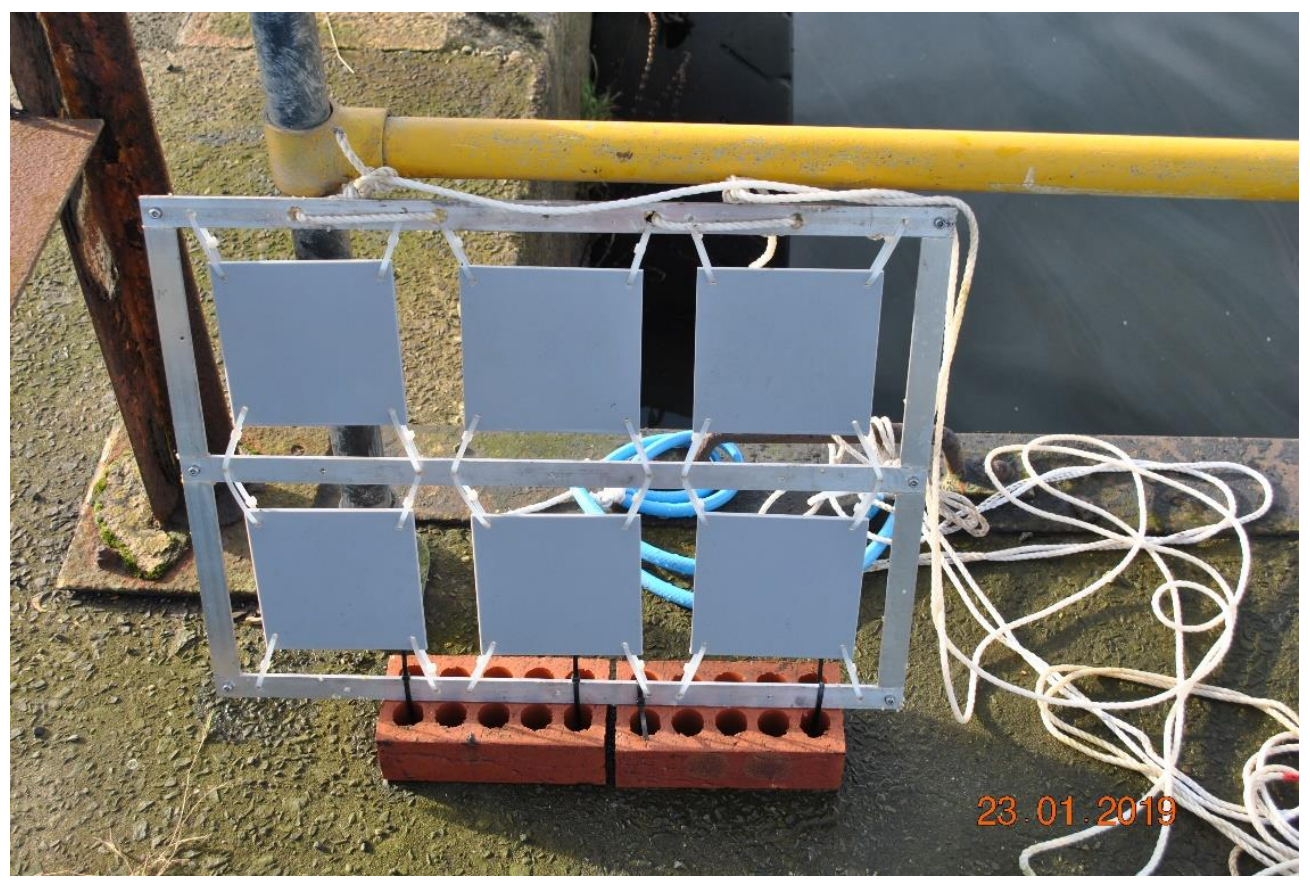

Cardiff: Week 24

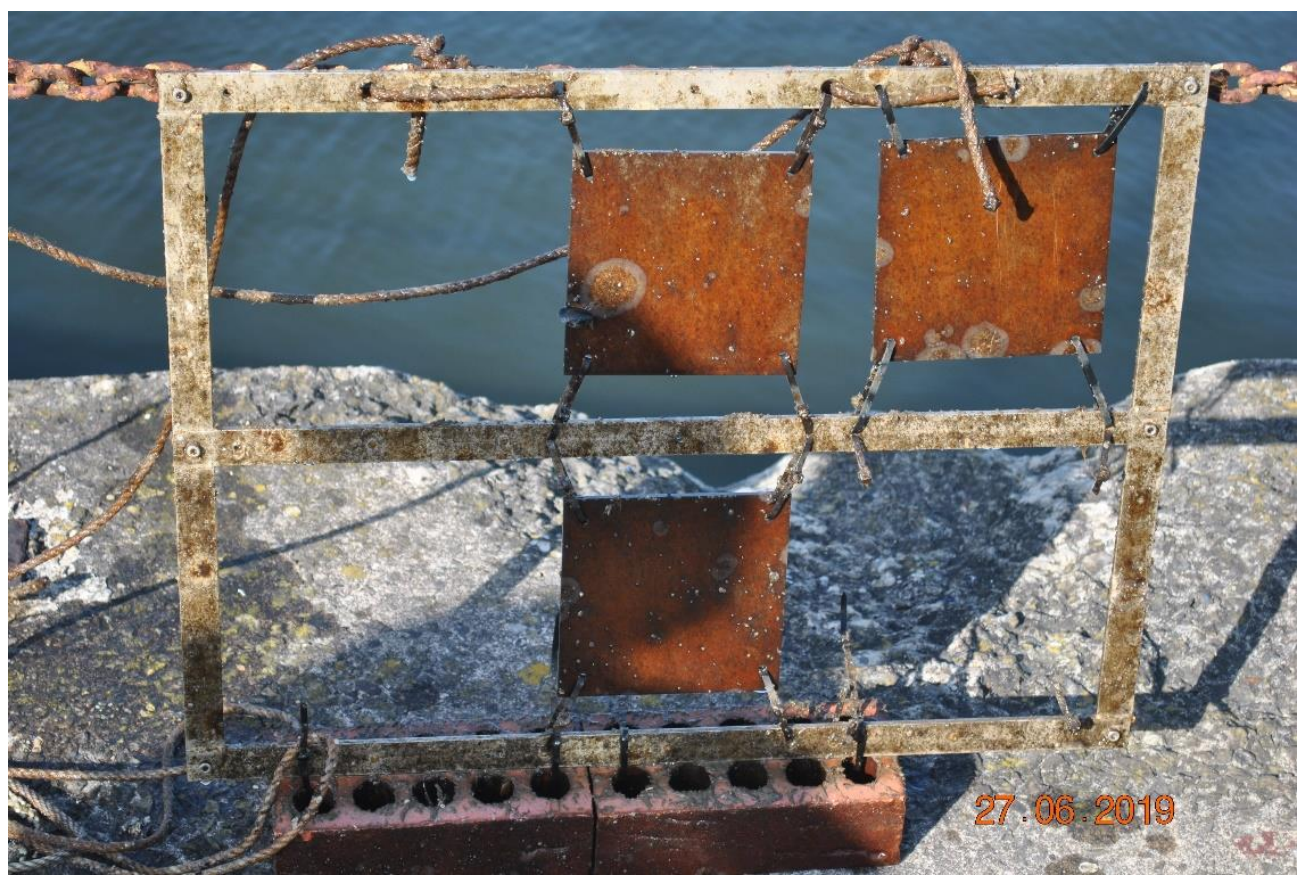


Cardiff: Week 36

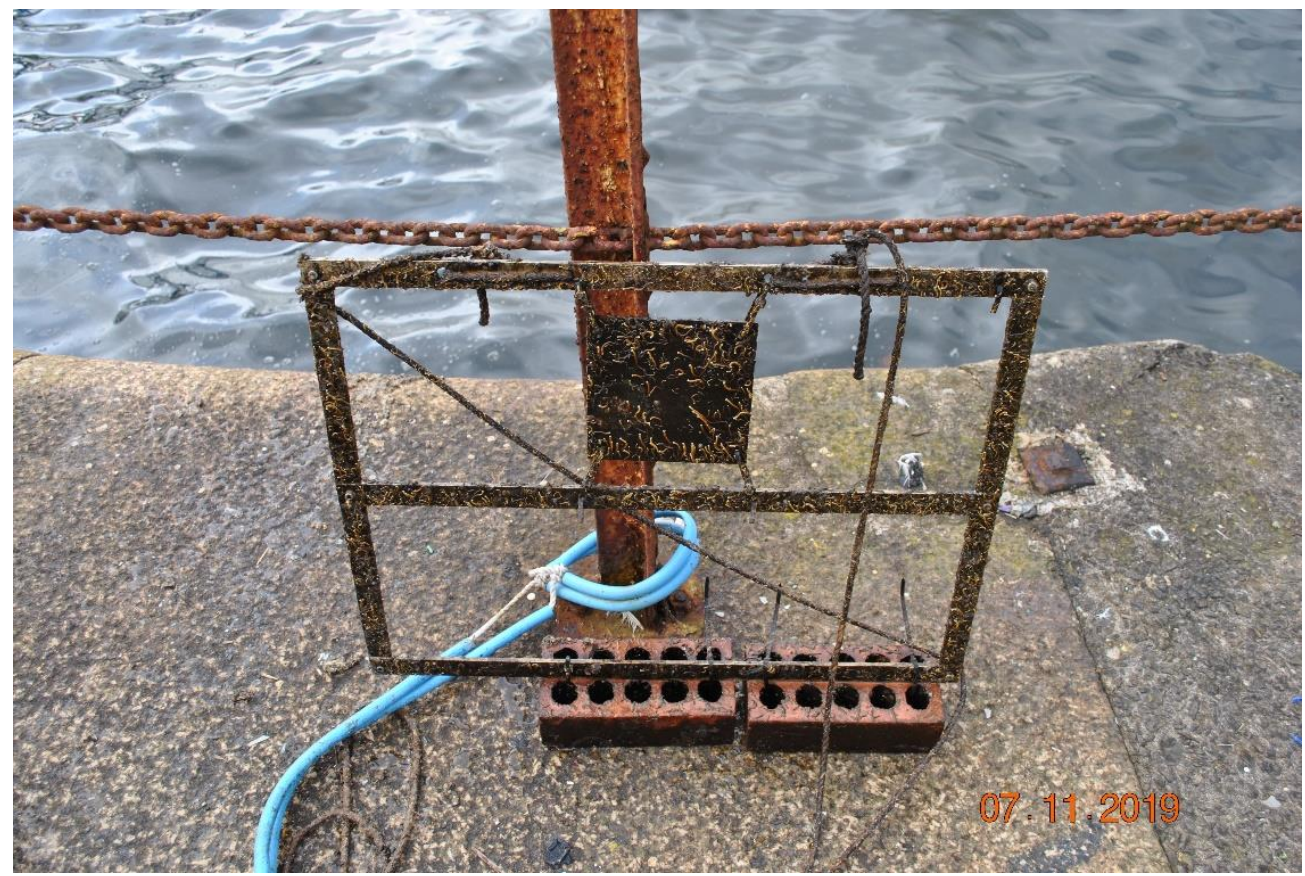

Barry: Week 6

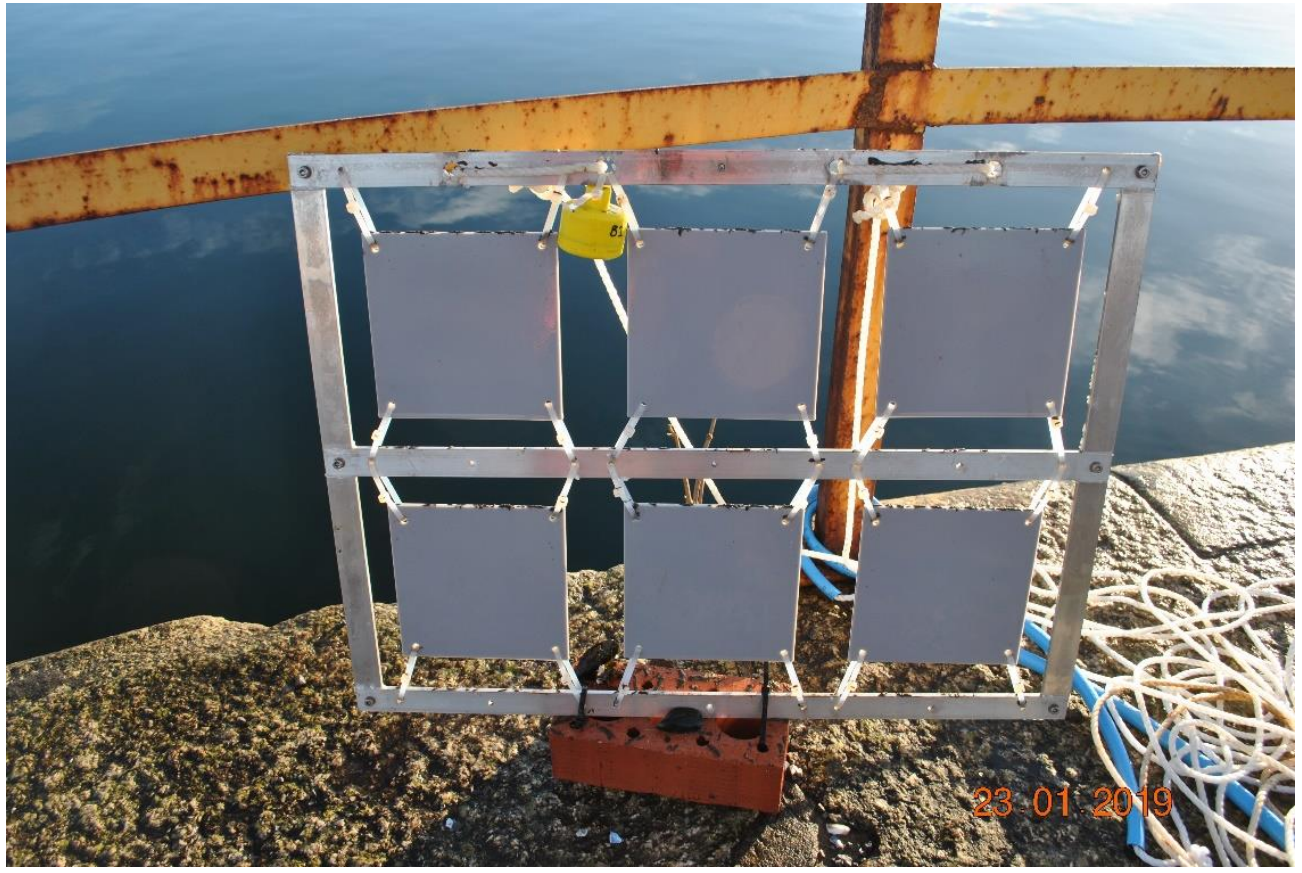


Barry: Week 24

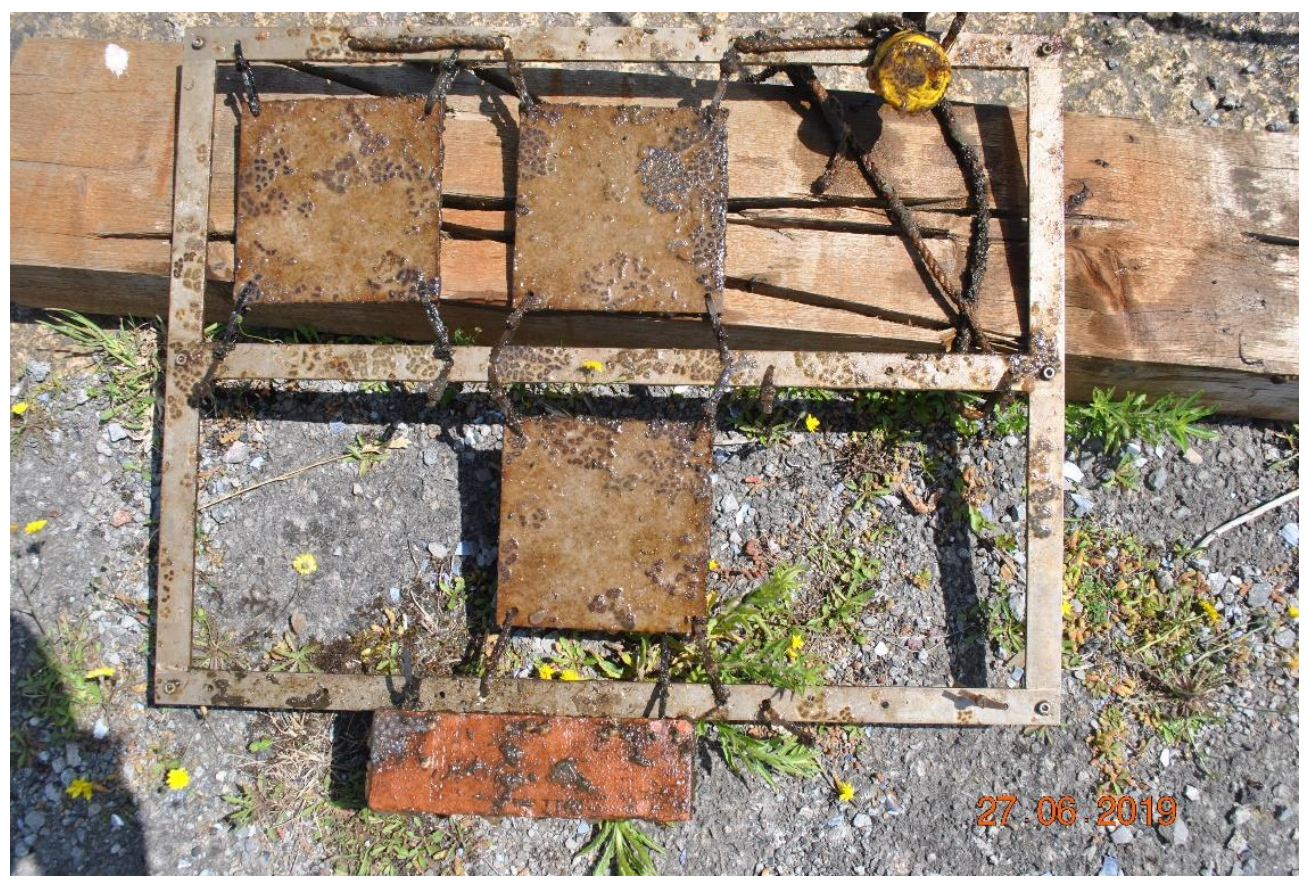

Barry: Week 36

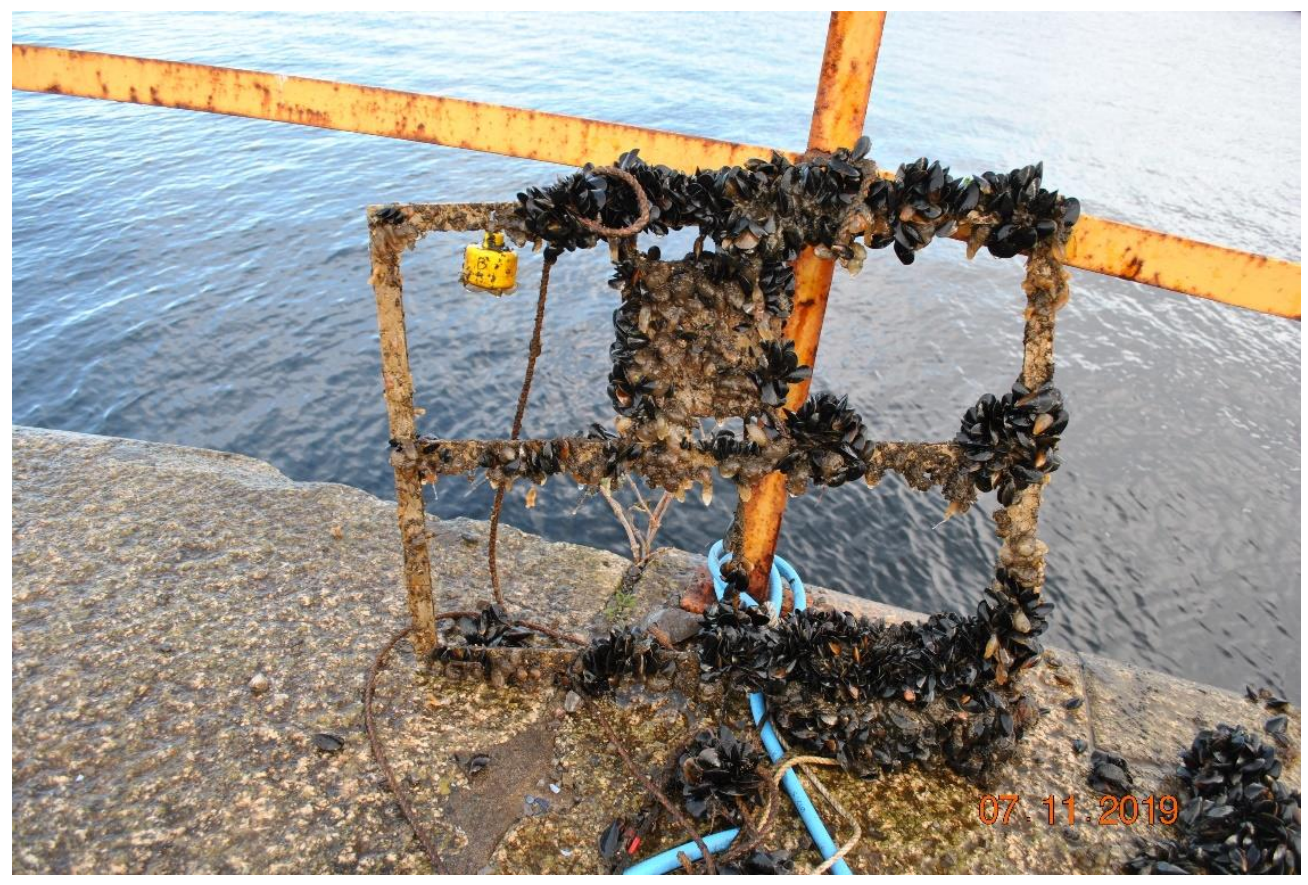


Port Talbot: Week 6

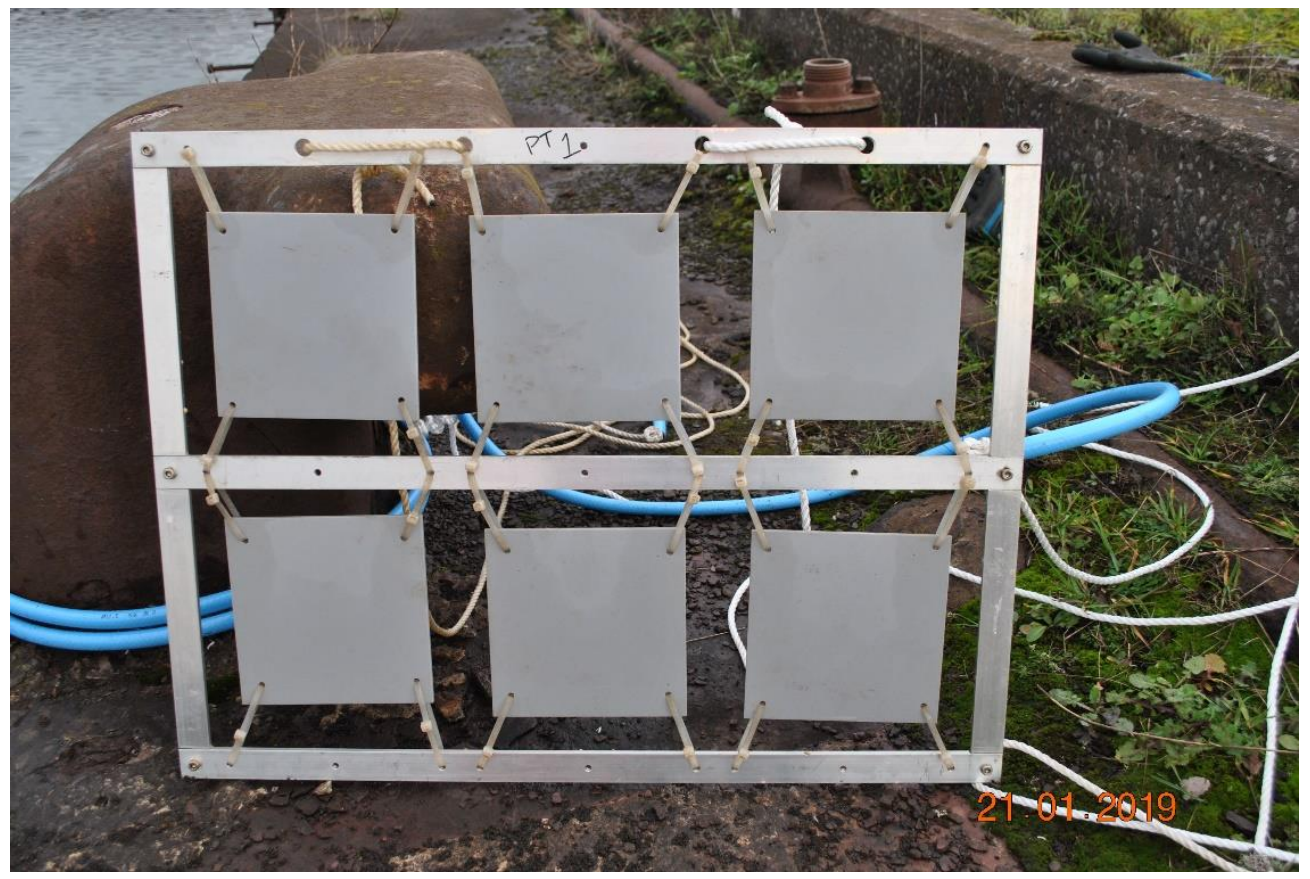

Port Talbot: Week 24

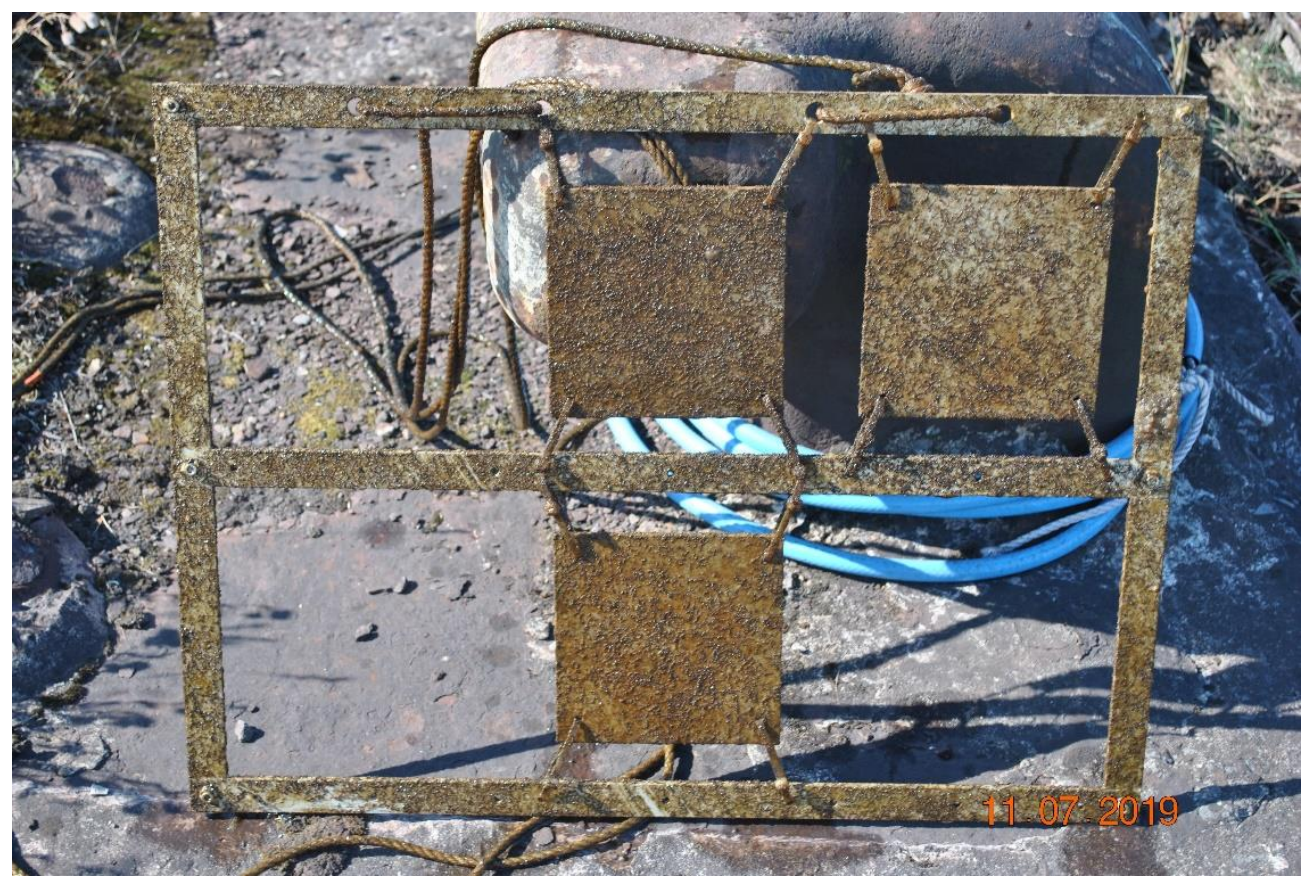


Port Talbot: Week 36

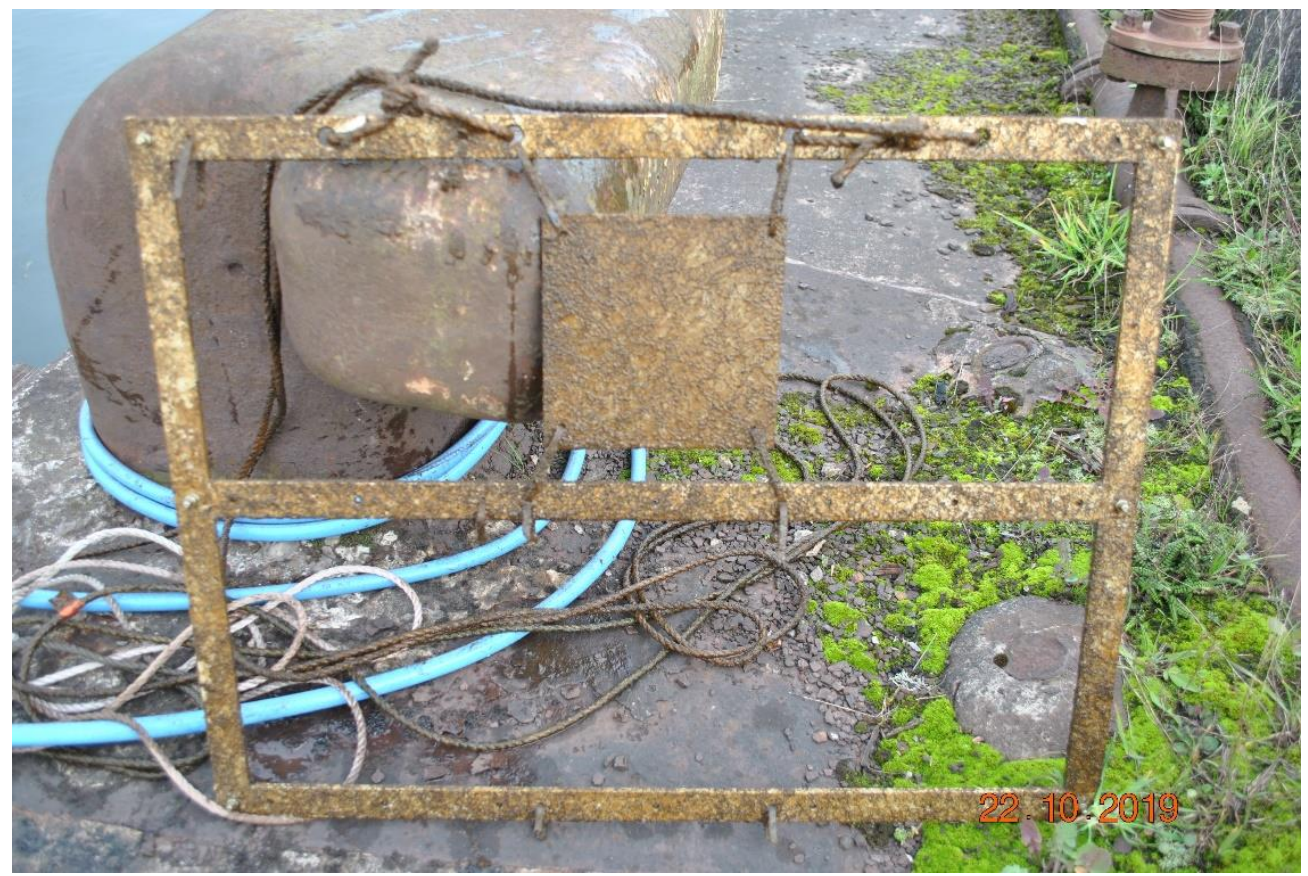

Swansea: Week 6

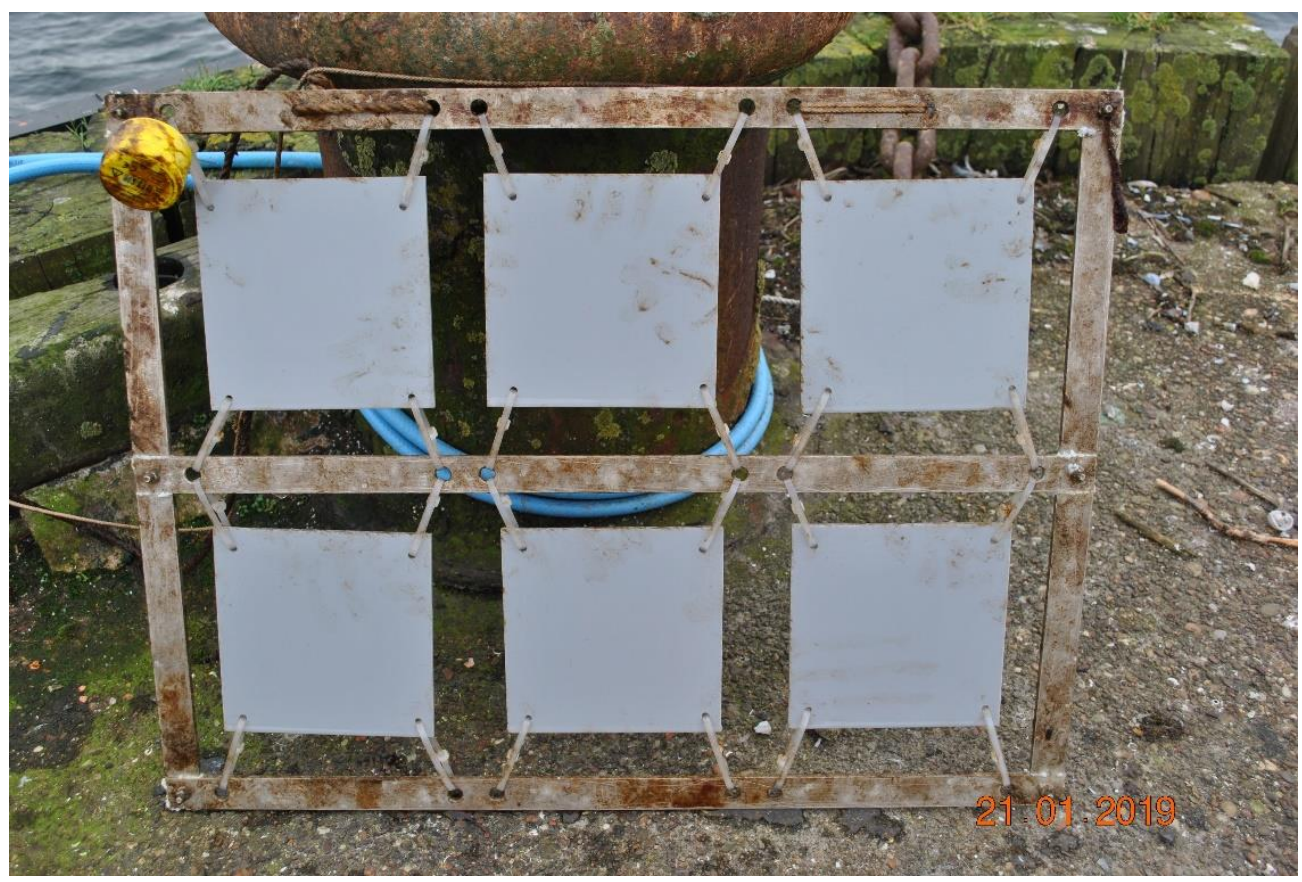


Swansea: Week 24

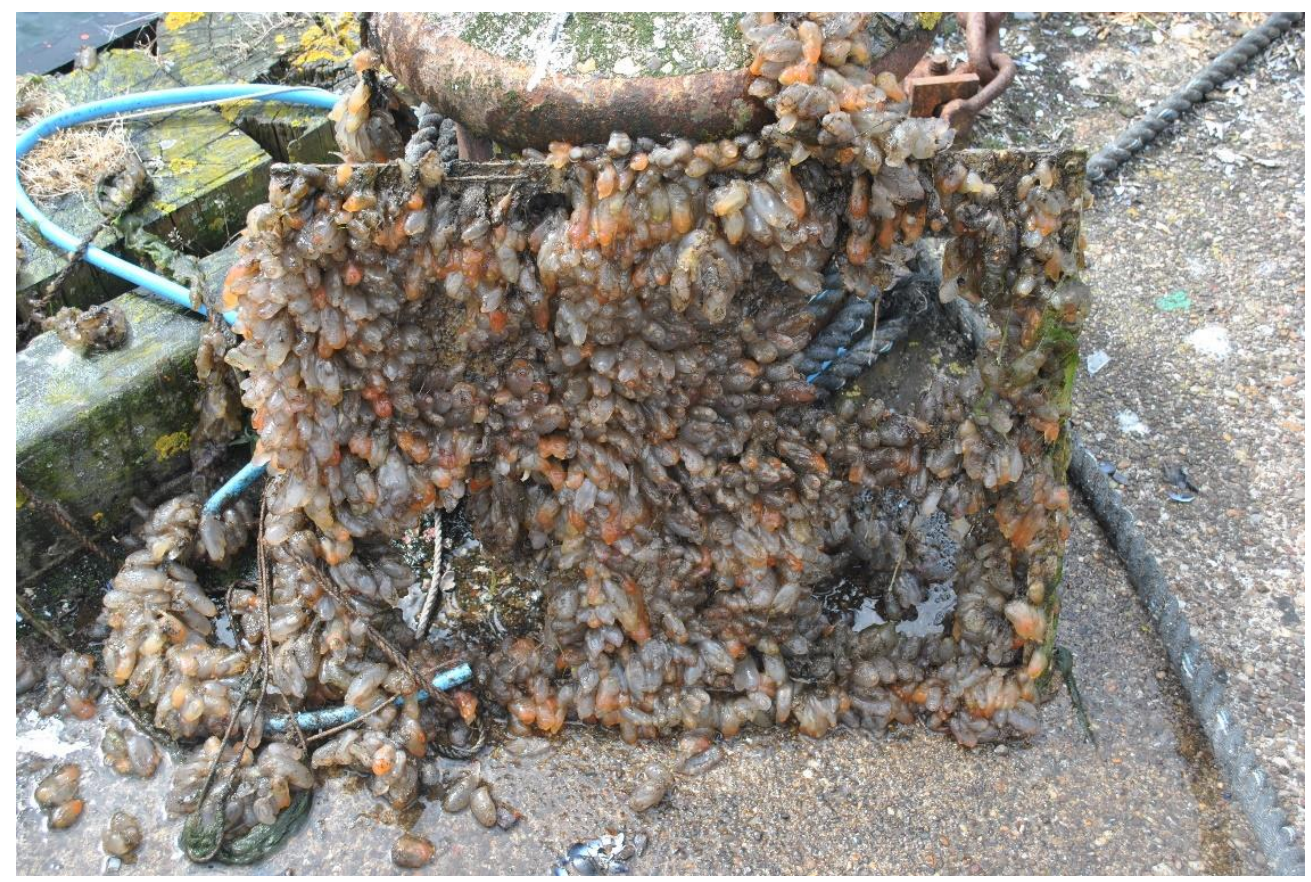

Swansea: Week 36

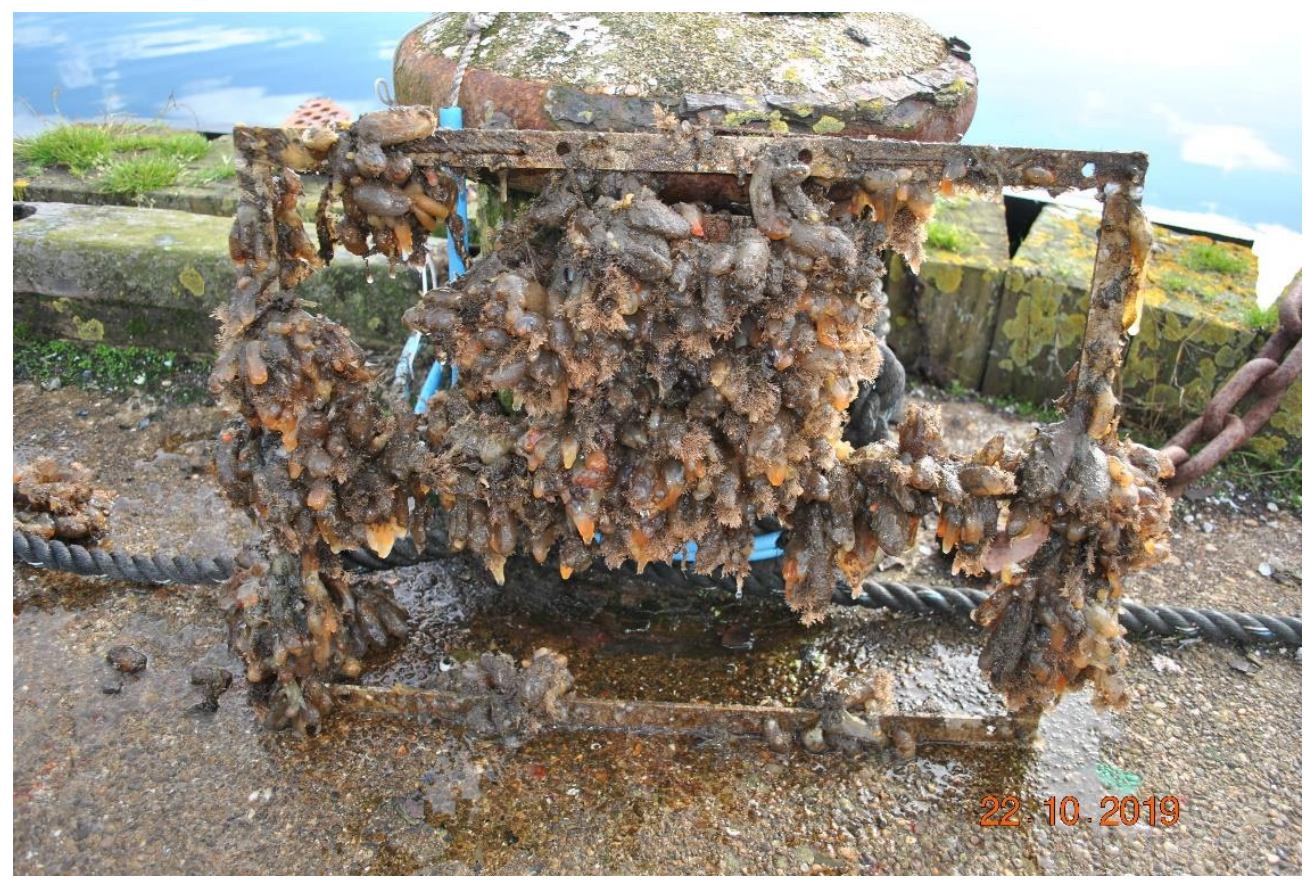


CHAPTER 3: Selection of photos showing colonisation upon retrieval of survey materials Newport: Mop head

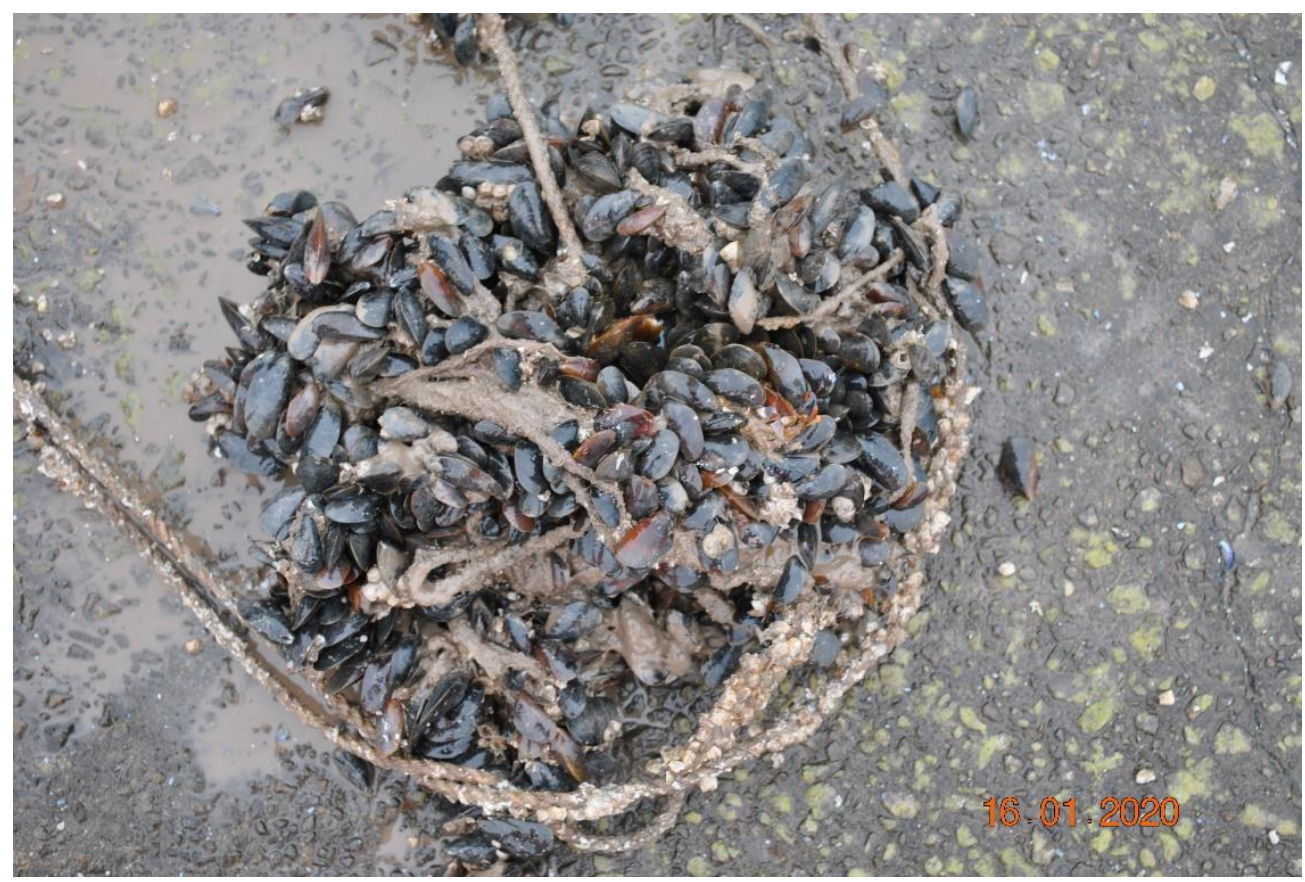

Newport: Wood

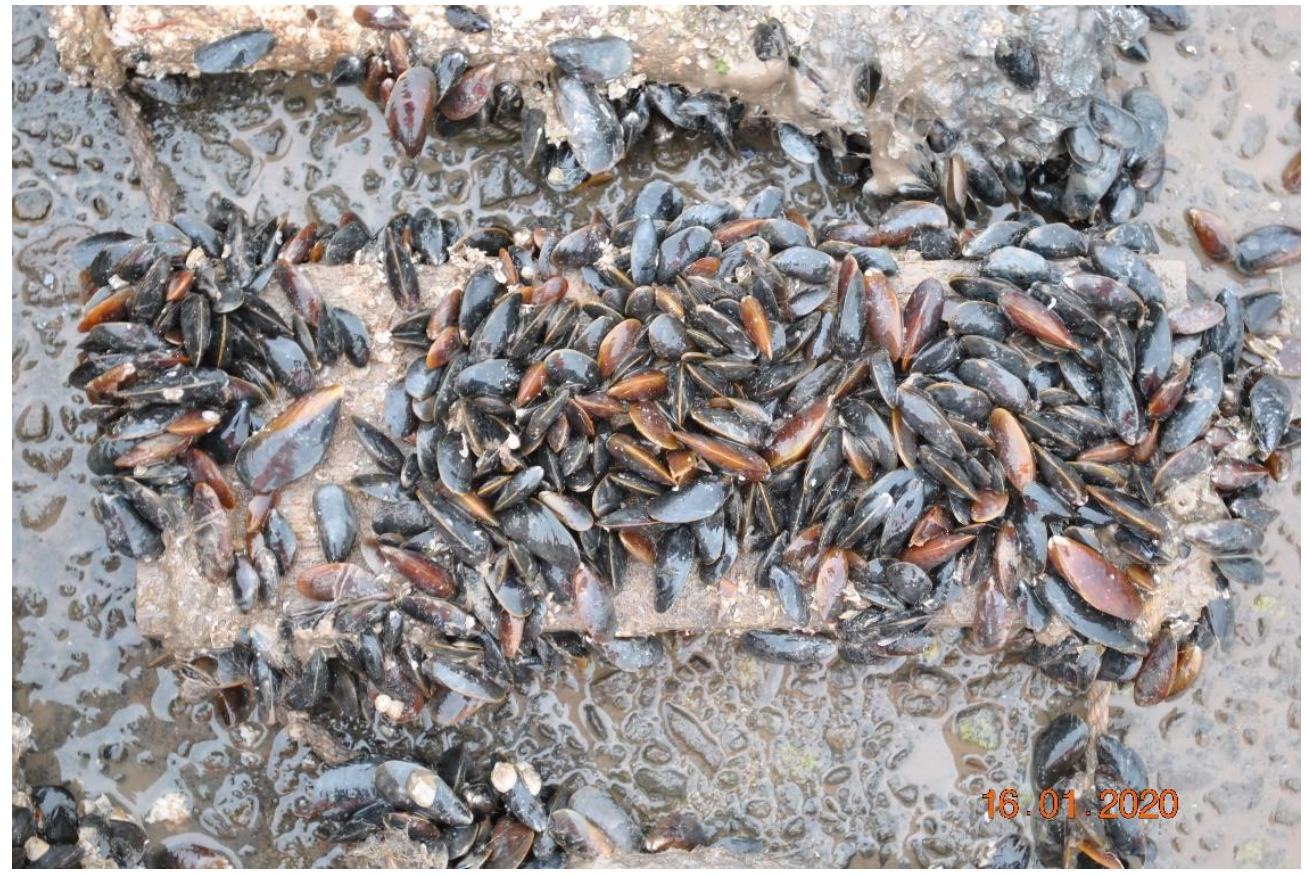


Newport: Polypropylene rope

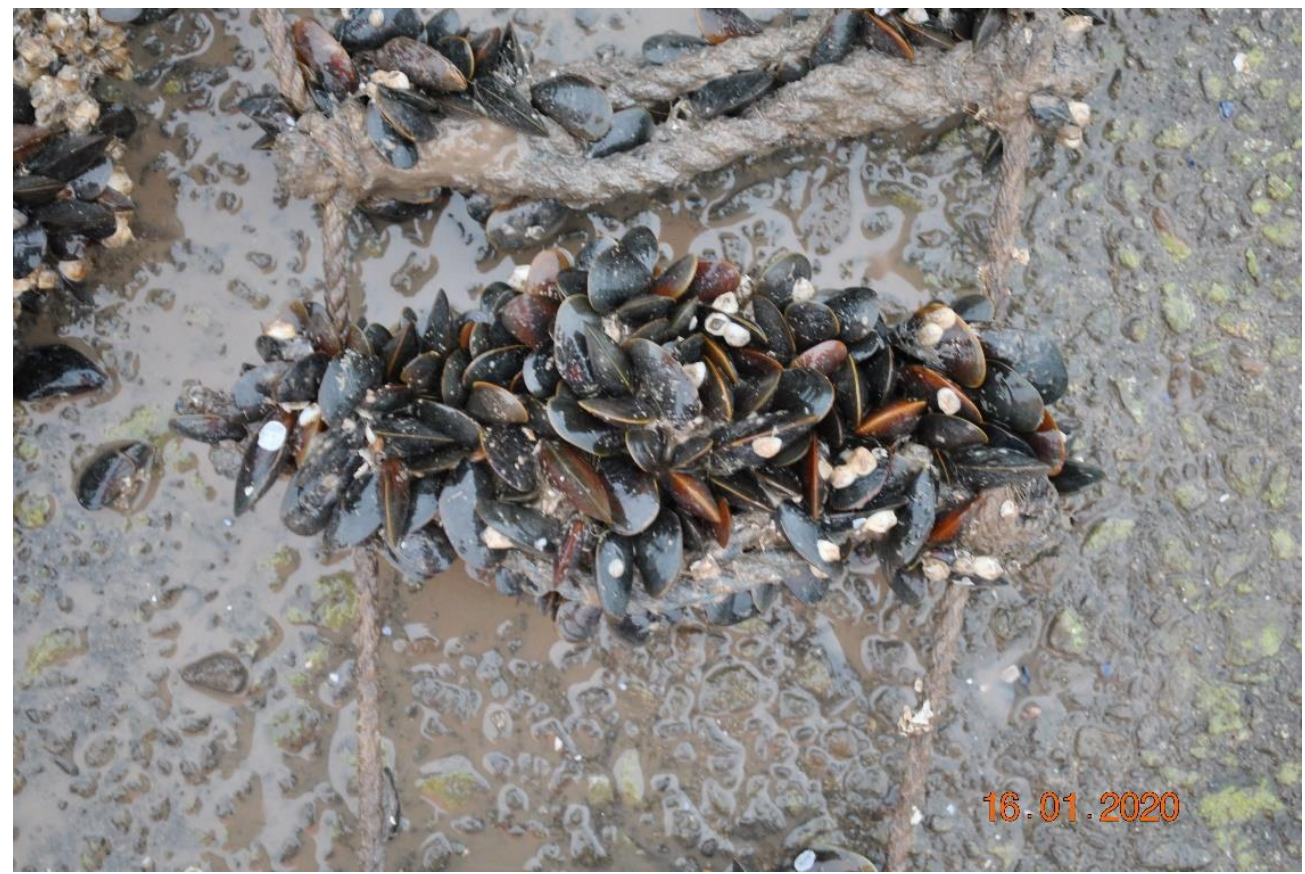

Cardiff: PVC (Unsanded)

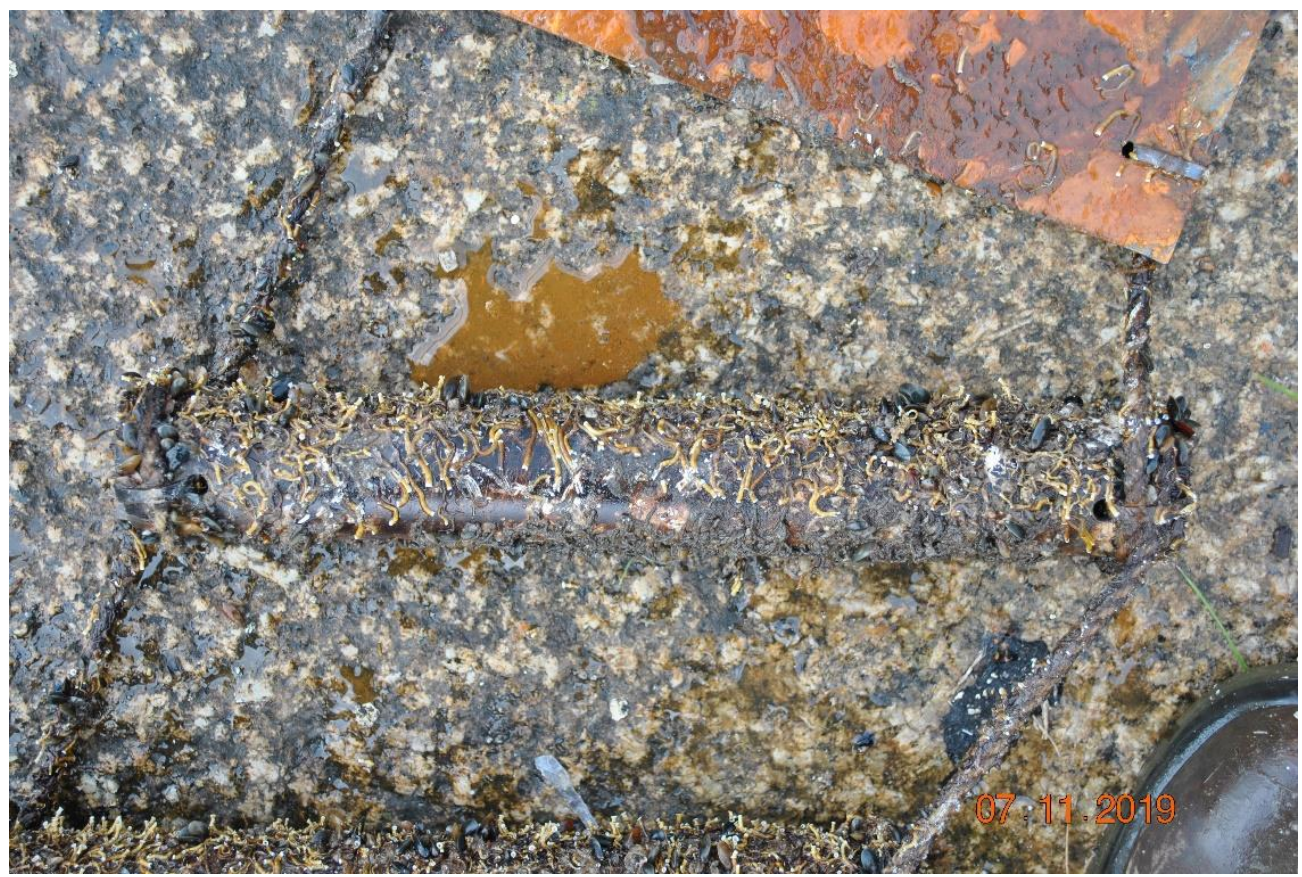


Cardiff: Wood

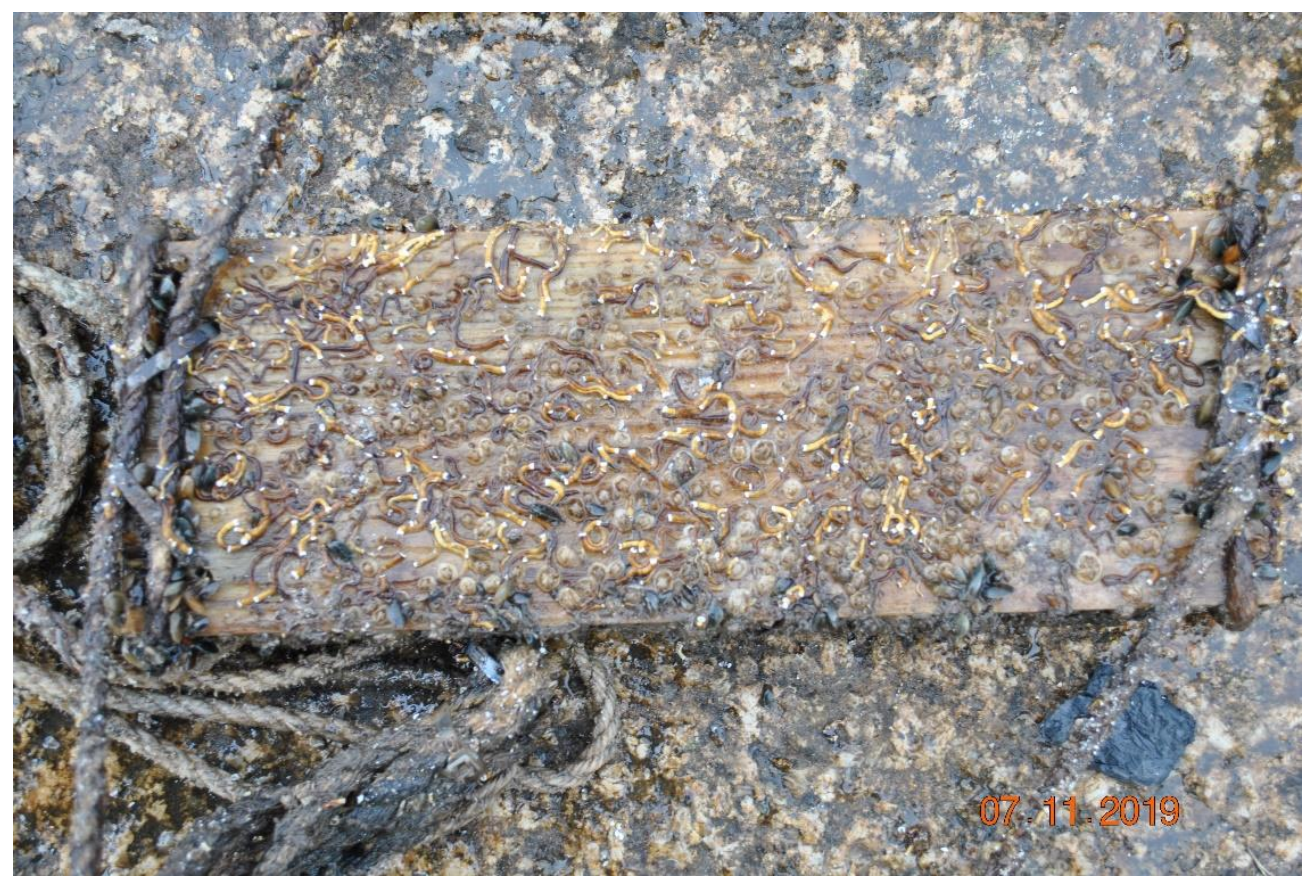

Cardiff: Steel

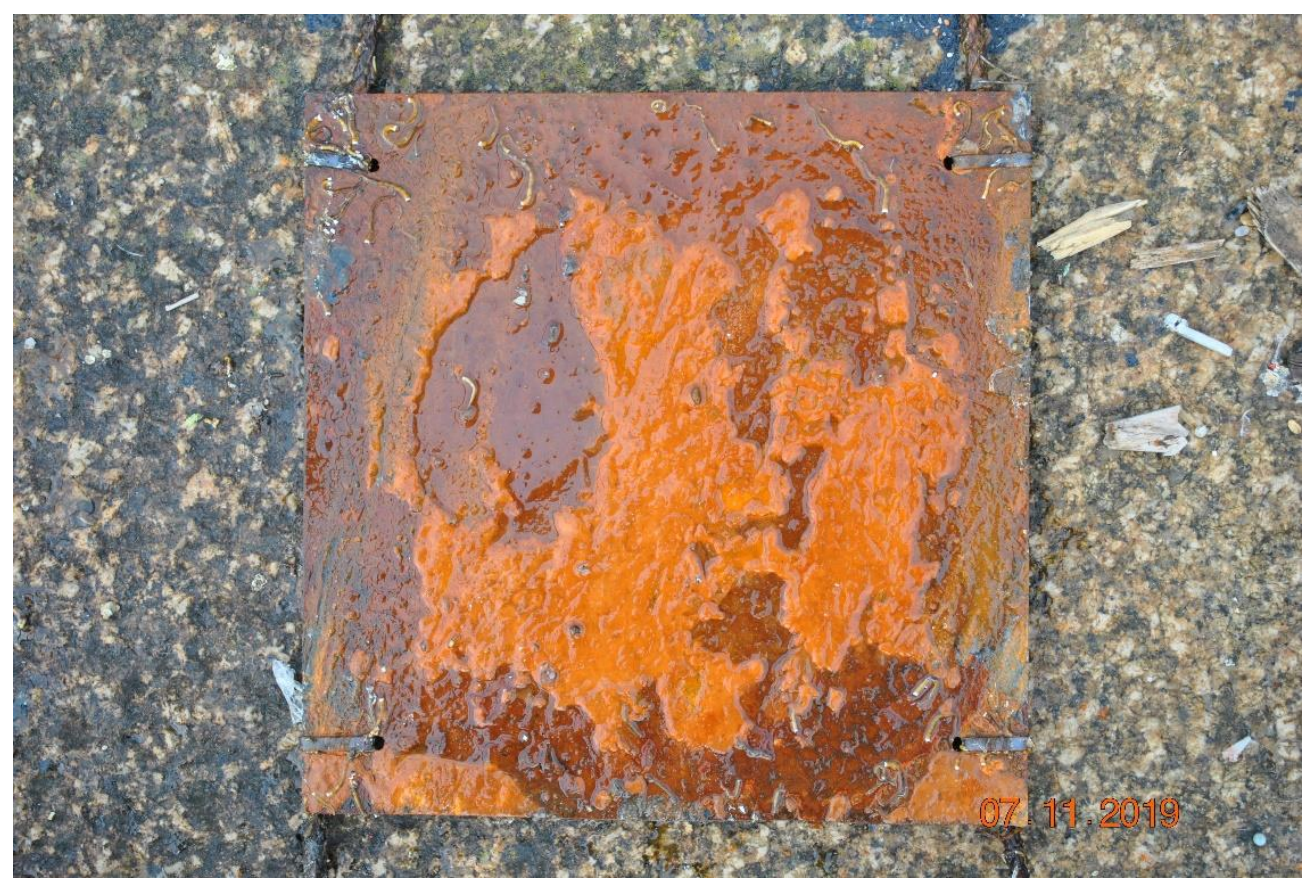




\section{Barry: Steel}

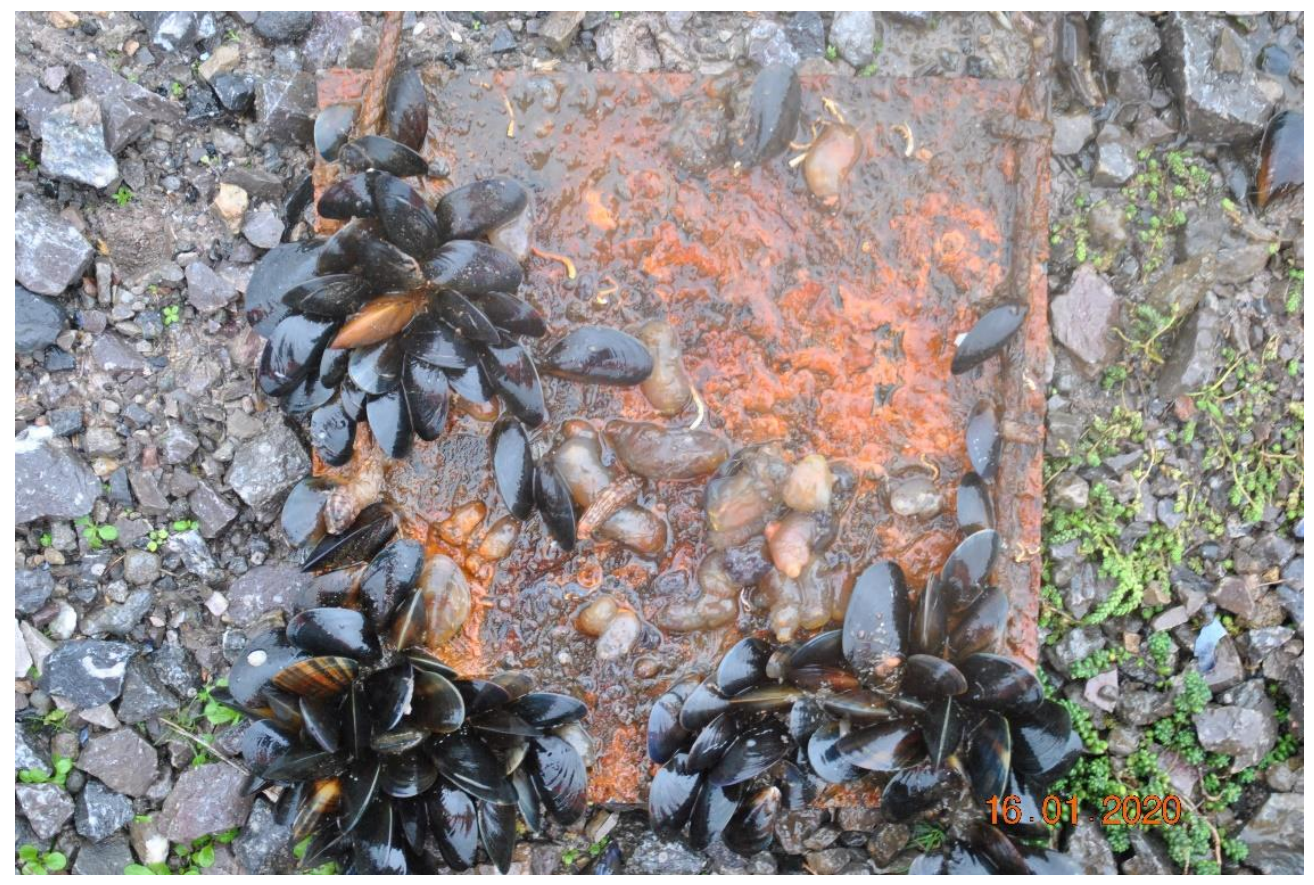

Barry: Brick

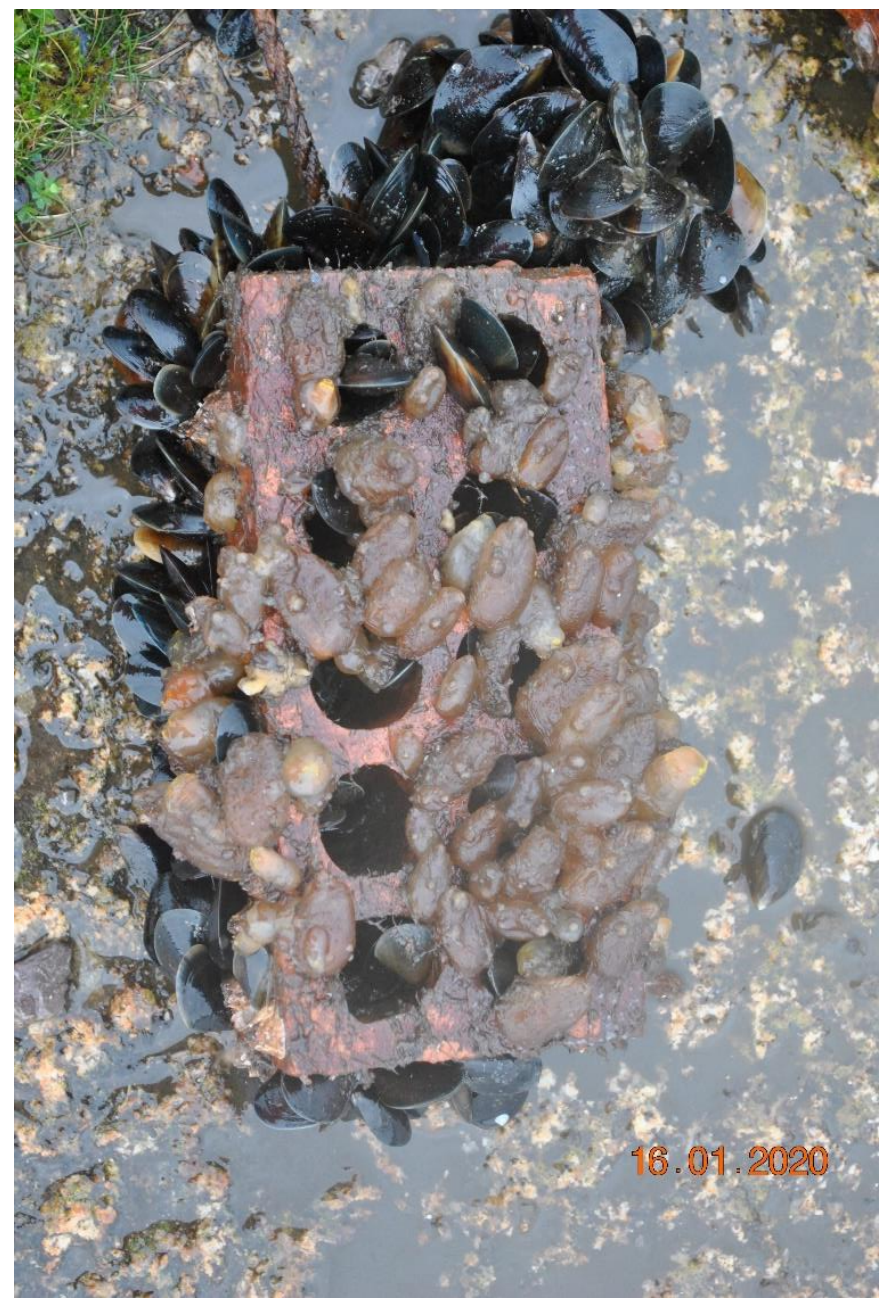


Barry: PVC (Sanded)

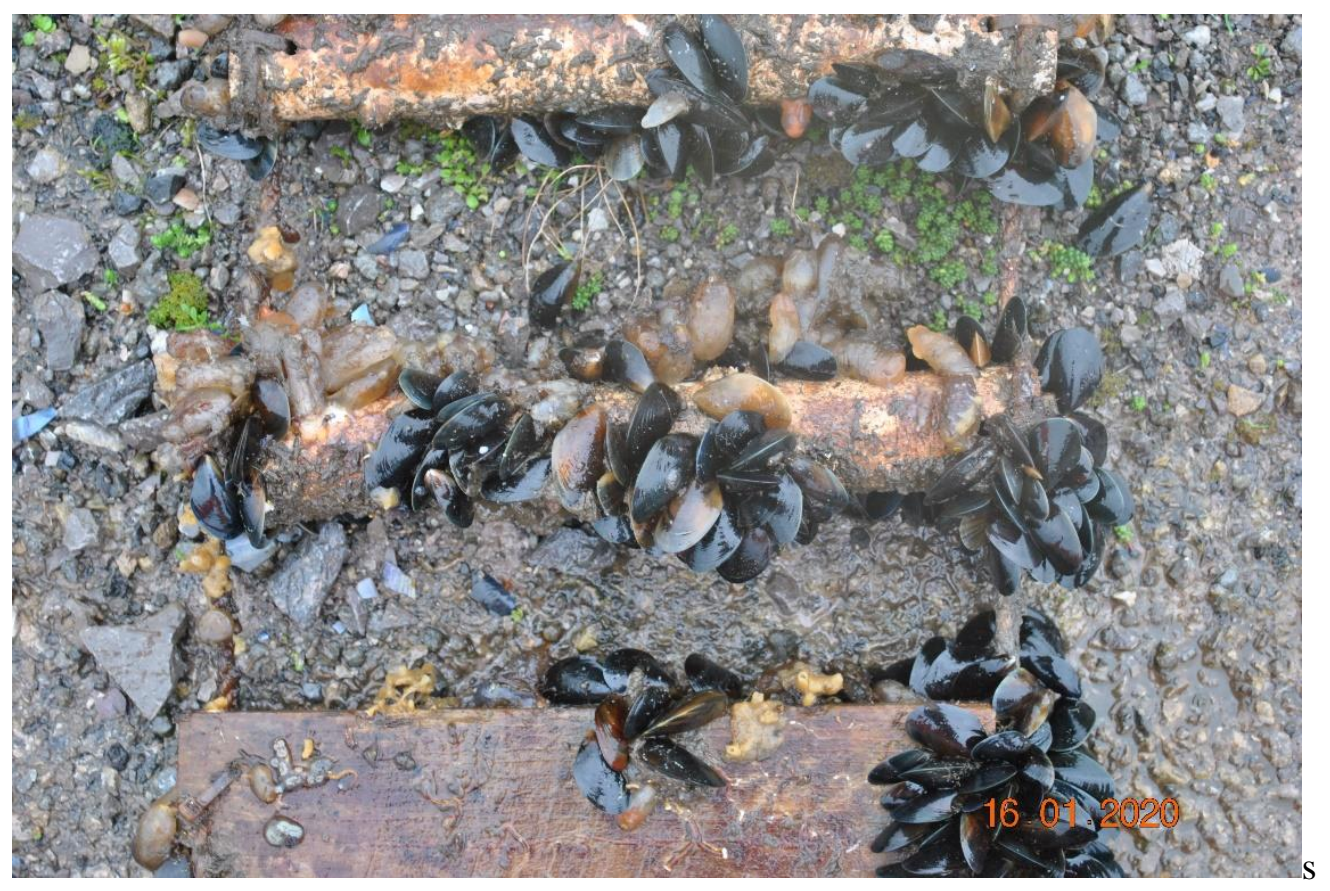

Port Talbot: All material

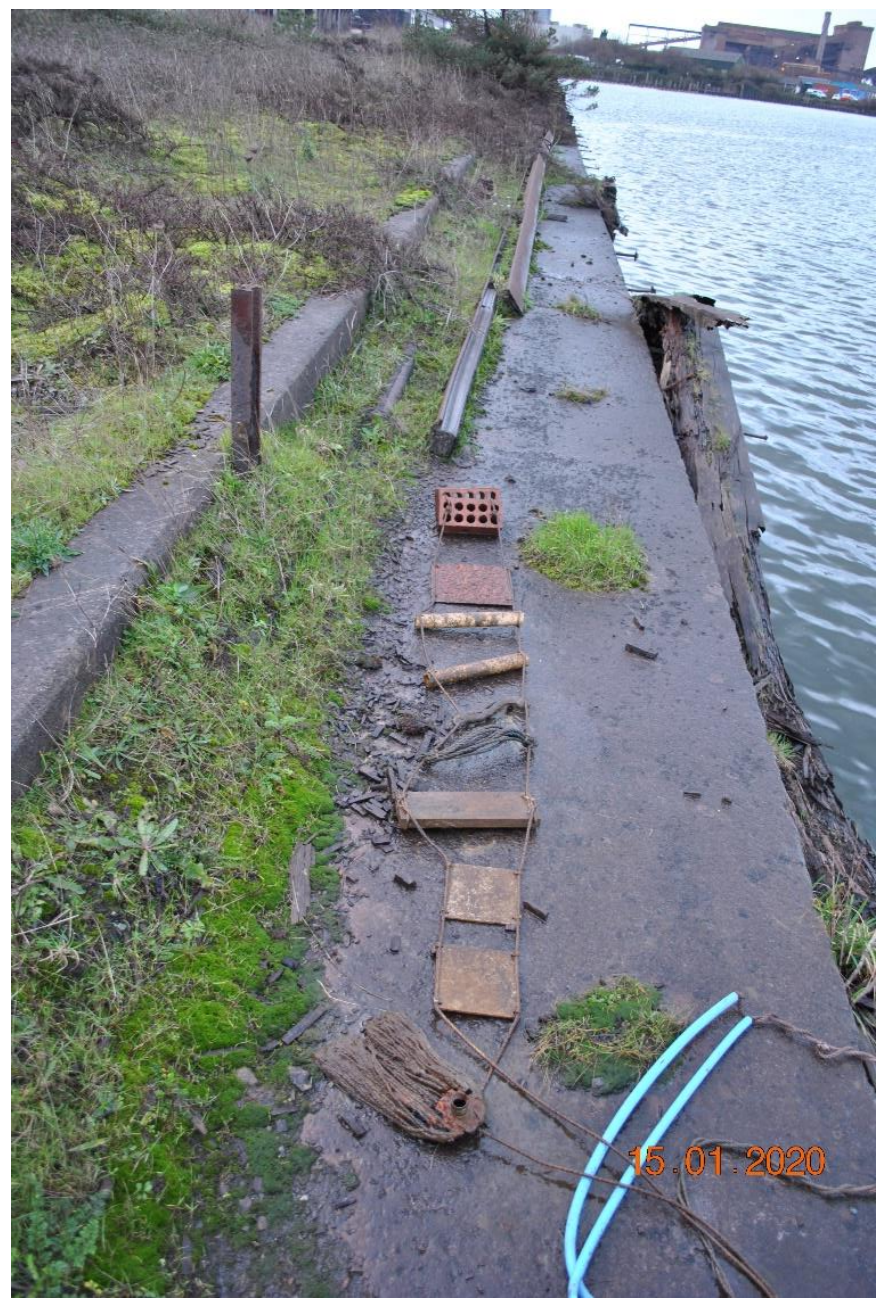


Port Talbot: PVC (Sanded)

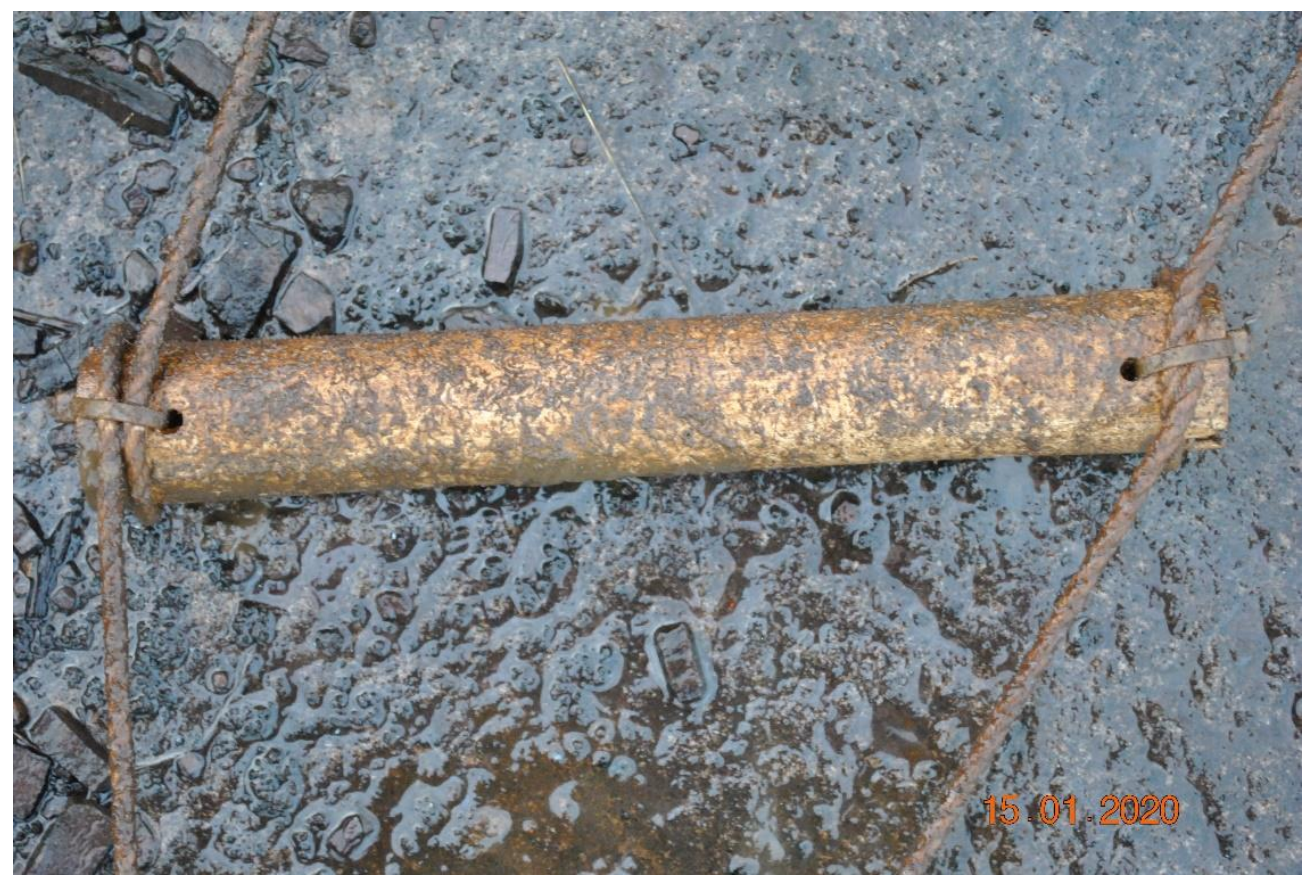

Port Talbot: Steel

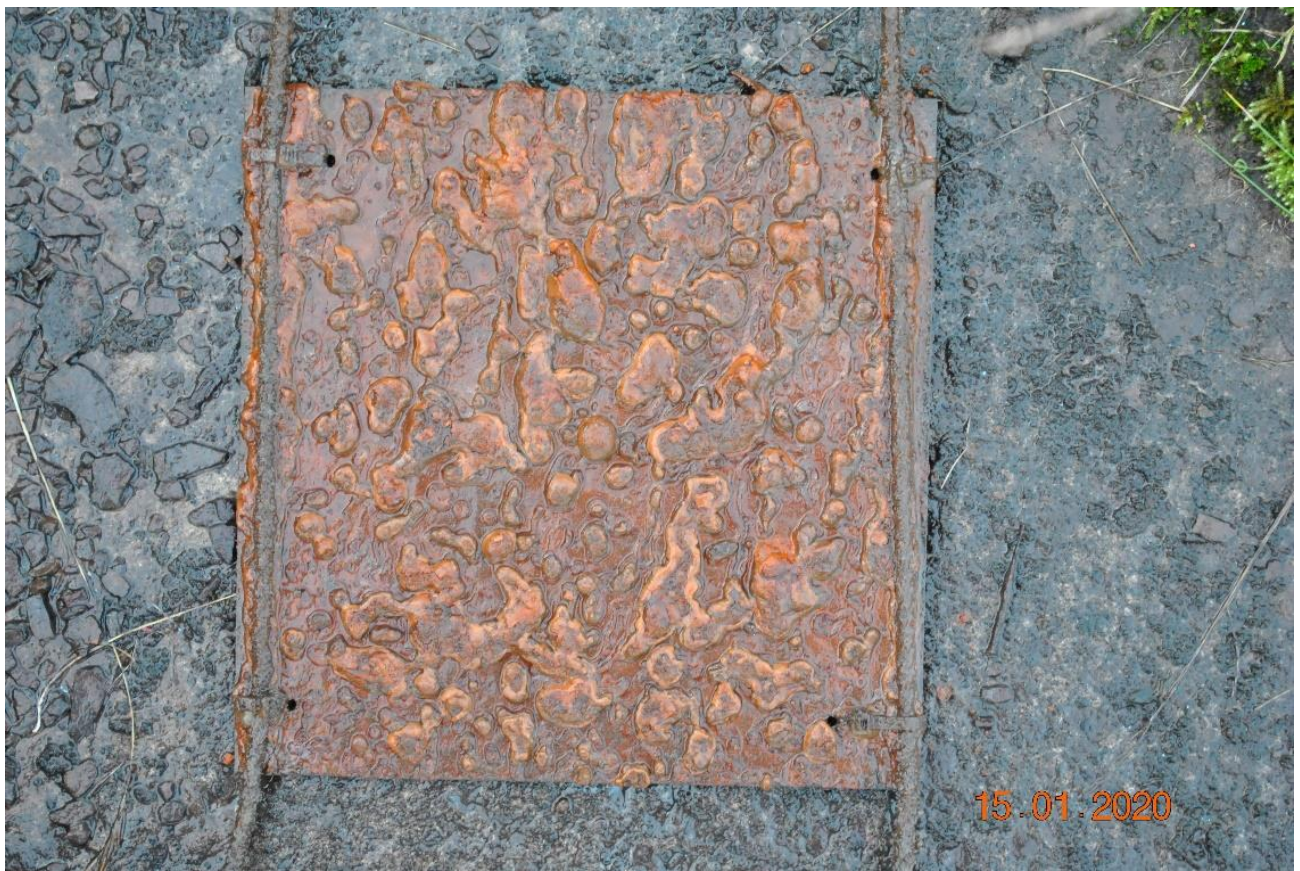


Swansea: Steel

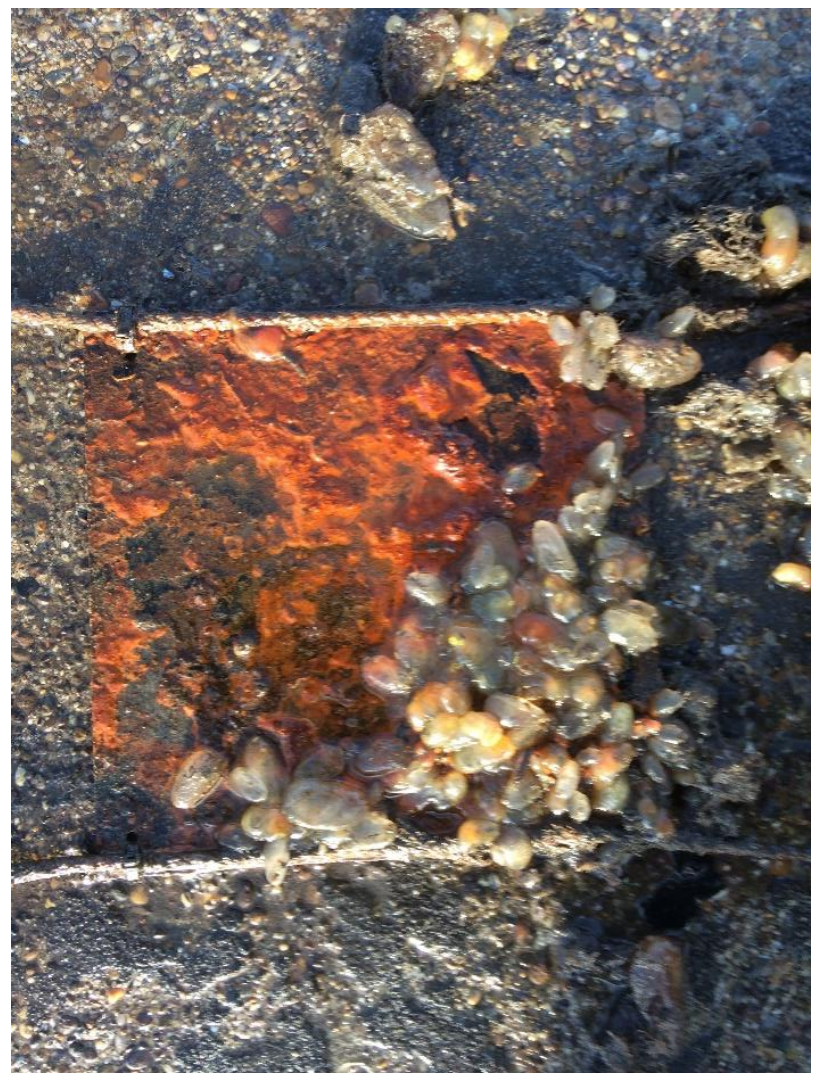

Swansea: Mop head

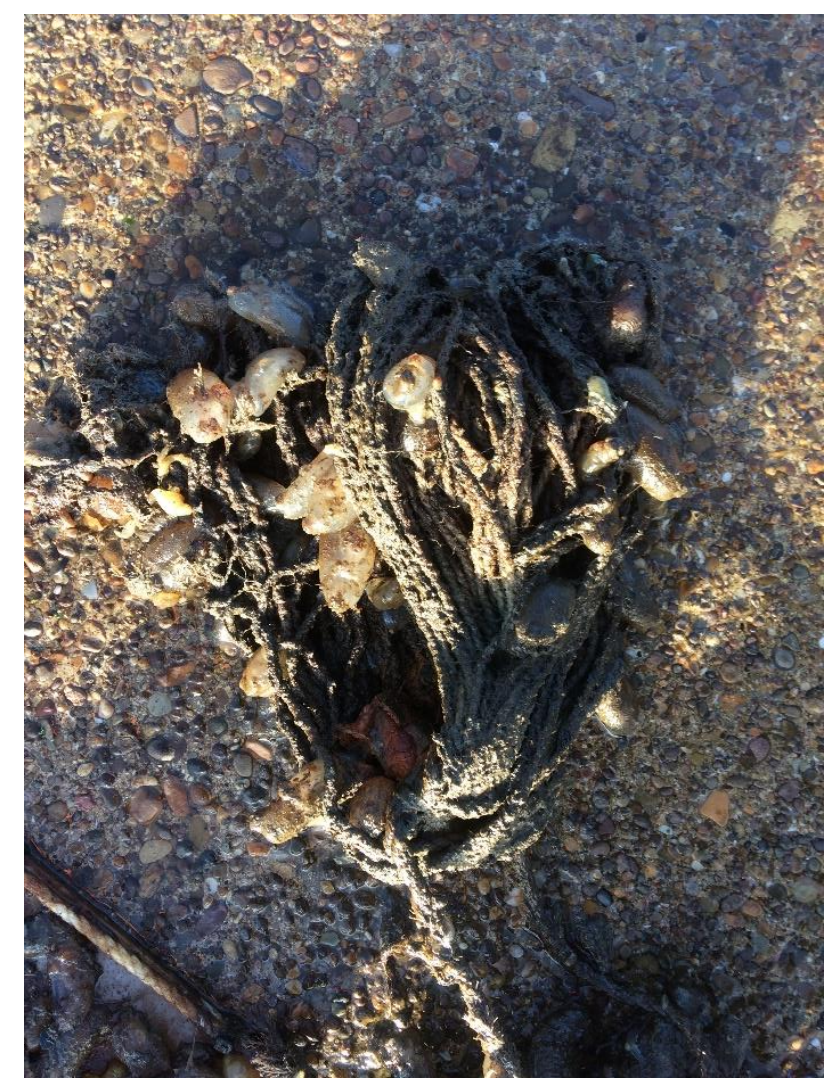


Swansea: PVC

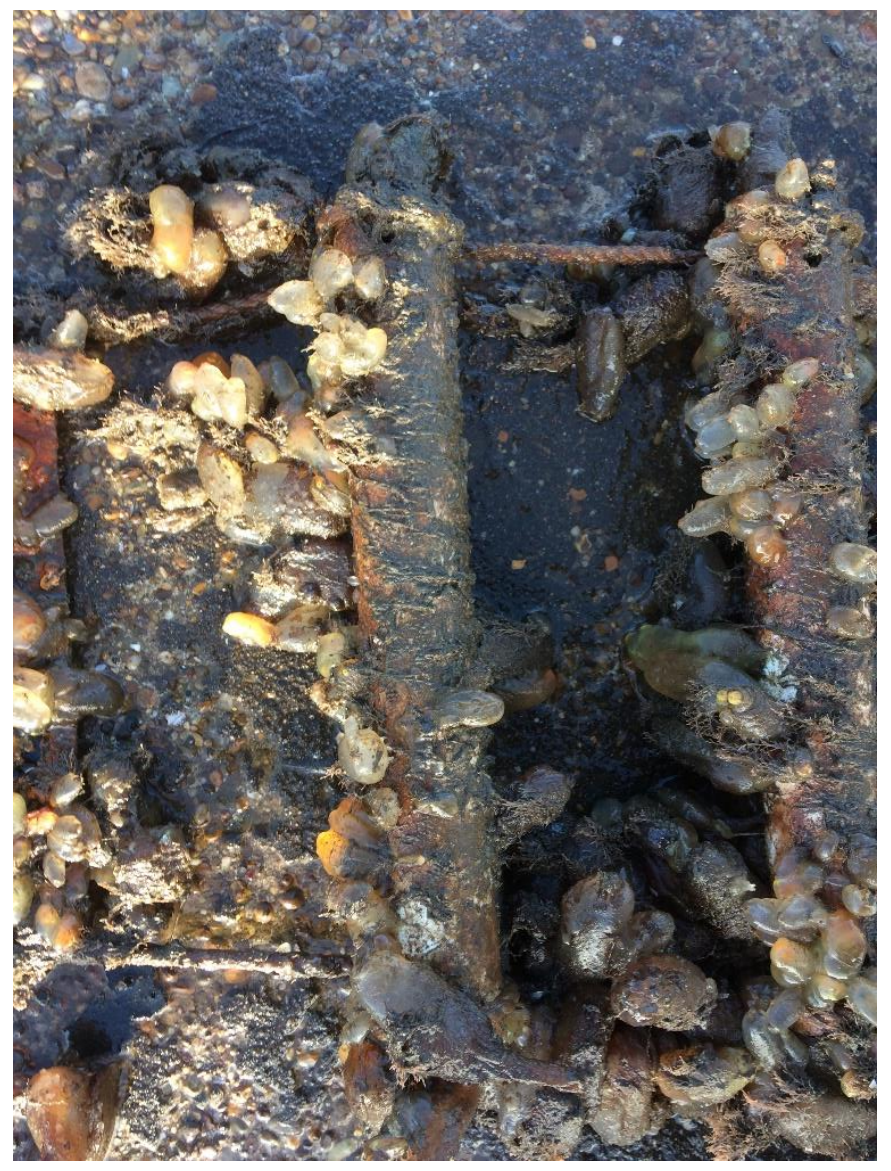

CHAPTER 4:

Plastid:

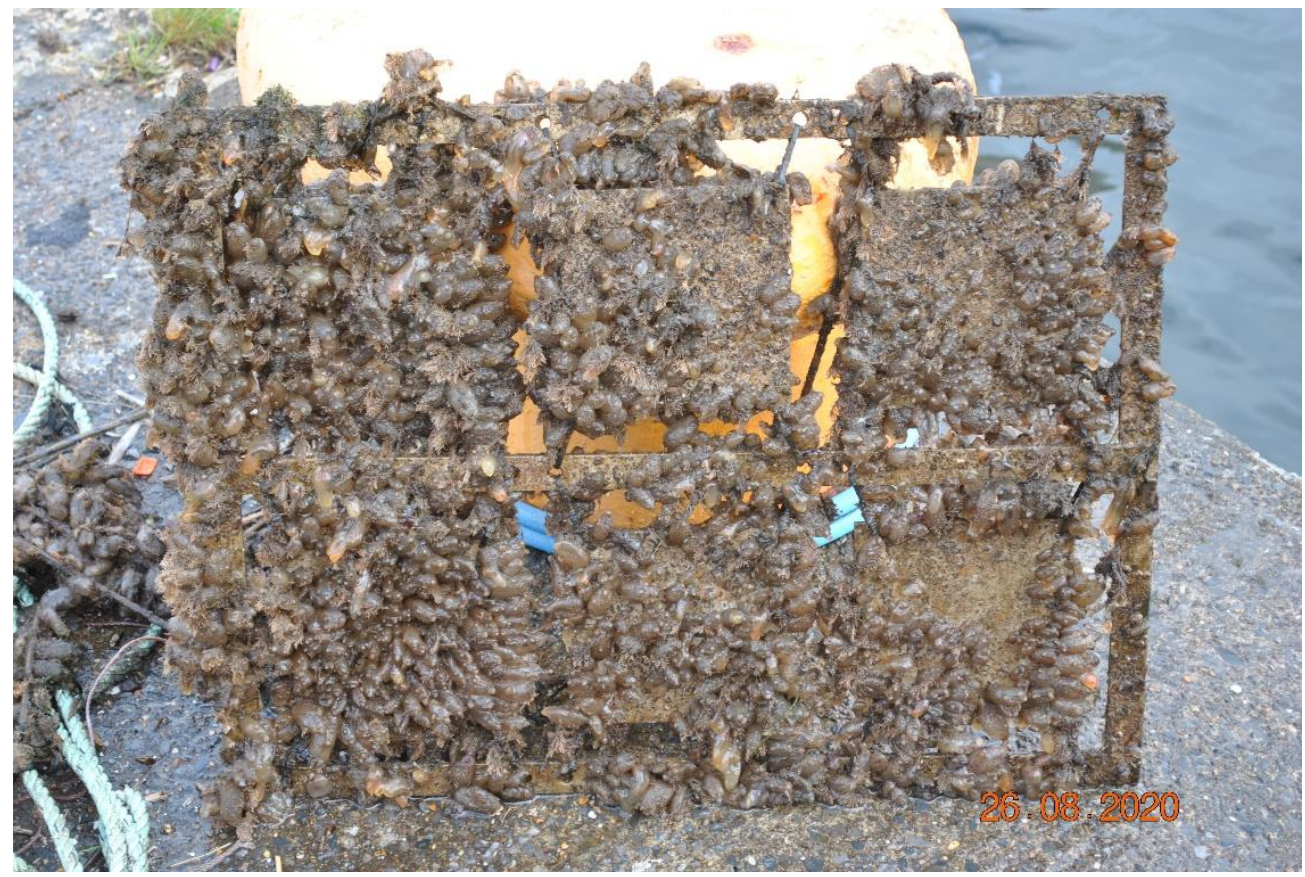


Slate:

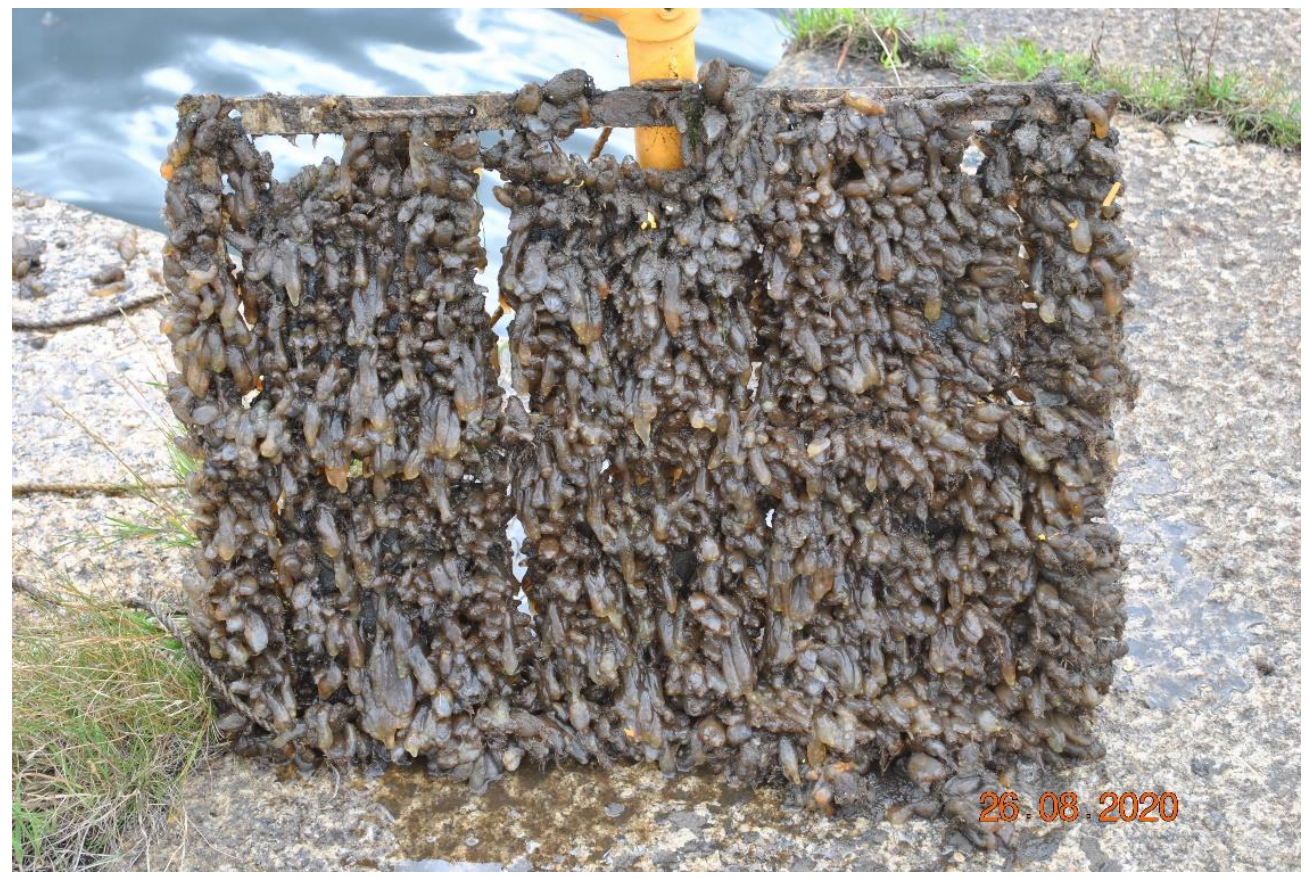




\section{APPENDIX IV: NON-NATIVE SPECIES FIELD IDENTIFICAITON GUIDE}

This appendix contains a non-native species (NNS) field guide for use by Associated British Ports (ABP) in future passive monitoring of NNS within their ports. The intended use of the field guide is for ABP employees who regularly spend time in and around the ports to familiarise themselves with the NNS known to be present within the region and report sightings to the appropriate port-based biosecurity manager. It is not expected that all NNS sightings will be recorded or indeed that all recordings will be accurate, however, the field guide is thought to be a valuable tool in achieving two key aims:

1. Increase the NNS monitoring capacity within ports

2. Engage port employees with the idea of NNS monitoring, hopefully driving a change in attitude toward environmental issues within the ports 


\section{A Guide to the Non-Native Species of South Wales Ports}

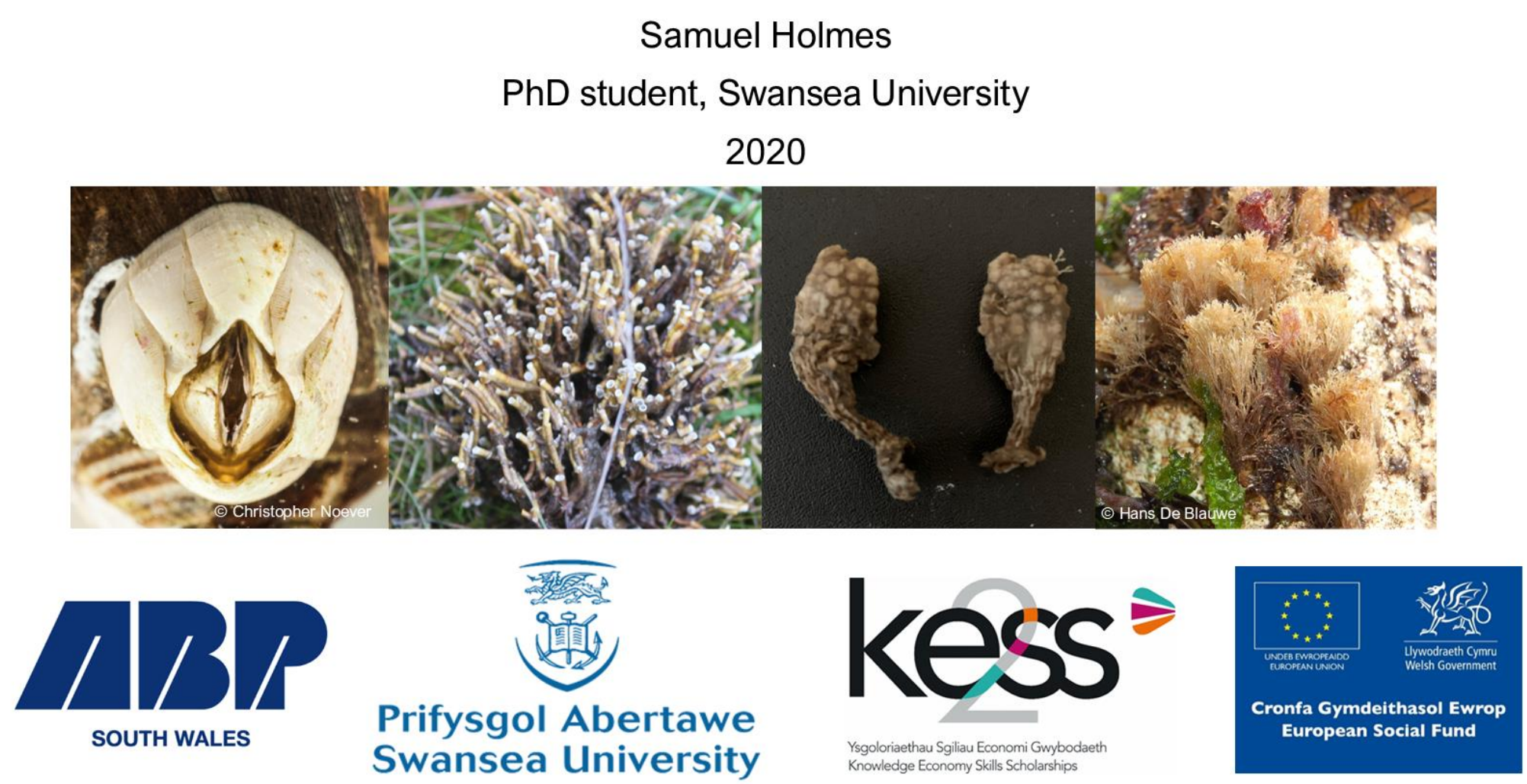




\section{Introduction:}

This guide has been developed following the deployment of field surveys within each of ABP's five South Wales ports (Newport, Cardiff, Barry, Port Talbot, Swansea). Surveys took place from 2018 into 2020 and were specifically focussed on detecting and identifying non-native species (NNS). Please note that the species listed in this guide are known to exist in the South Wales ports and may be present along with additional, currently unknown NNS in other ABP ports. The intended use of this guide for ABP staff is to both familiarise themselves with the known NNS from within the ports they frequently operate within, but to also assist in monitoring the spread of NNS between ports and the potential introduction of new NNS into the ports.

\section{Sightings of NNS in new ports/ areas of ports must be reported to your local Biosecurity Manager \& Environmental Coordinator}

NNS are known to cause huge financial losses in the shipping industry through the interference in processes and damaging of equipment. NNS can also pose a significant threat to our native UK species, including rare and endangered species. Every aspect of NNS monitoring can be vital in understanding how to limit the risk of future invasions and protect our native ecosystems and our industry. 


\section{Using the guide:}

This is a typical information sheet for each NNS:

Latin species name (Common name, if available)

List of ports this species

known to be present in
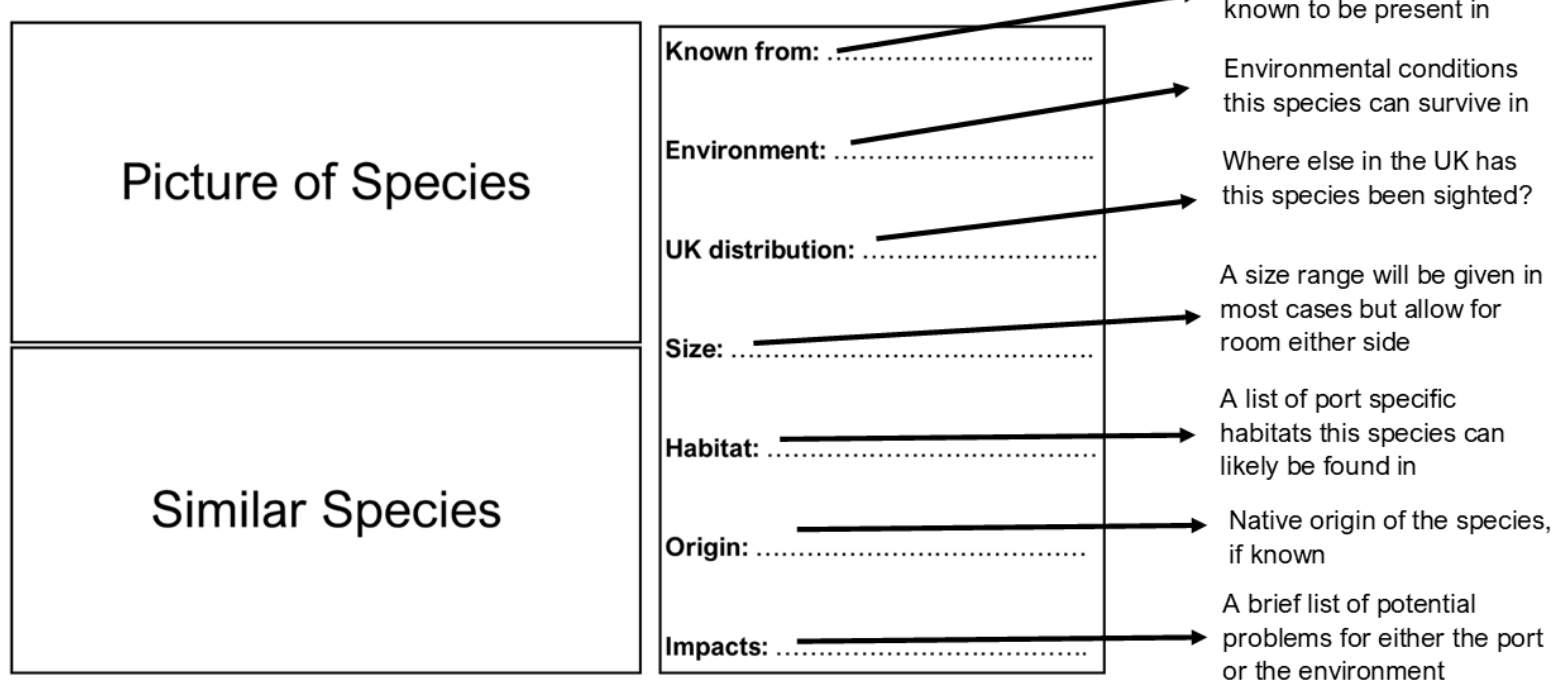

To identify NNS focus on the PICTURE, SIZE and HABITAT. Use the information on which port the species is known to be present in and the environmental conditions to assist you, but don't rely heavily on this information as species can often turn up in surprising locations! And remember, always take photos when you can. 


\section{Information on the South Wales ports:}

One of the key findings from surveying each of the five South Wales ports was the variation in species recorded from each port. This was due in a large part to the difference in salinity between ports, shown in the table below.

\begin{tabular}{|l|l|}
\hline Port & Salinity range \\
\hline Newport & $8-16$ (Brackish) \\
\hline Cardiff & $1-12$ (Brackish) \\
\hline Barry & $22-24$ (Brackish) \\
\hline Port Talbot & $0.1-0.3$ (Freshwater) \\
\hline Swansea & $28-30$ (Marine) \\
\hline
\end{tabular}

These ports are all located within around 55 miles of coastline, and display significant differences in the environmental conditions and species present. This is a good illustration of exactly why it is important to report sightings of non-native species from all ports, as it cannot be assumed that any two ports will share the same species. 


\section{Amphibalanus improvisus (Bay barnacle)}

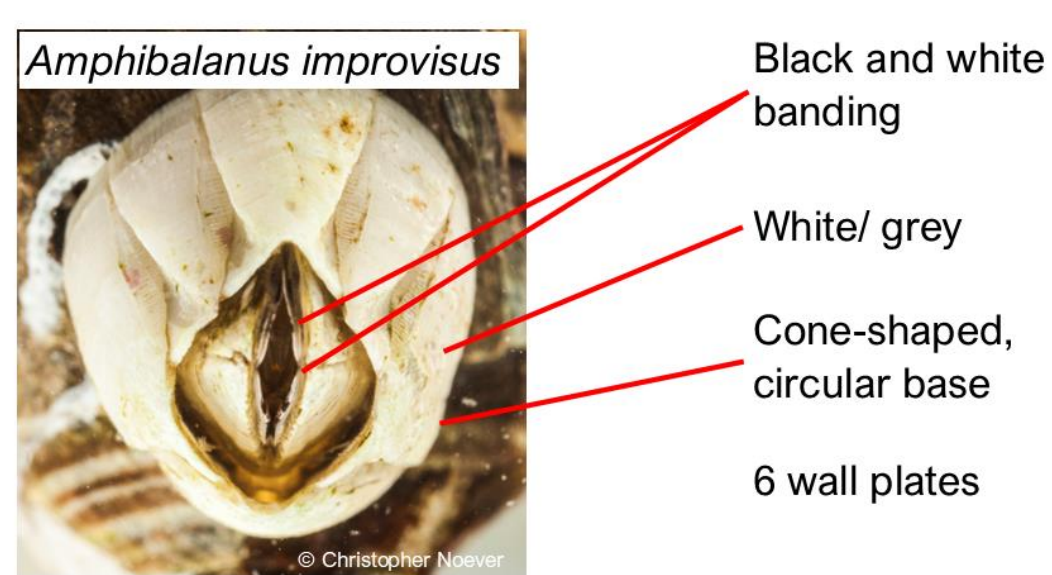

Similar species:

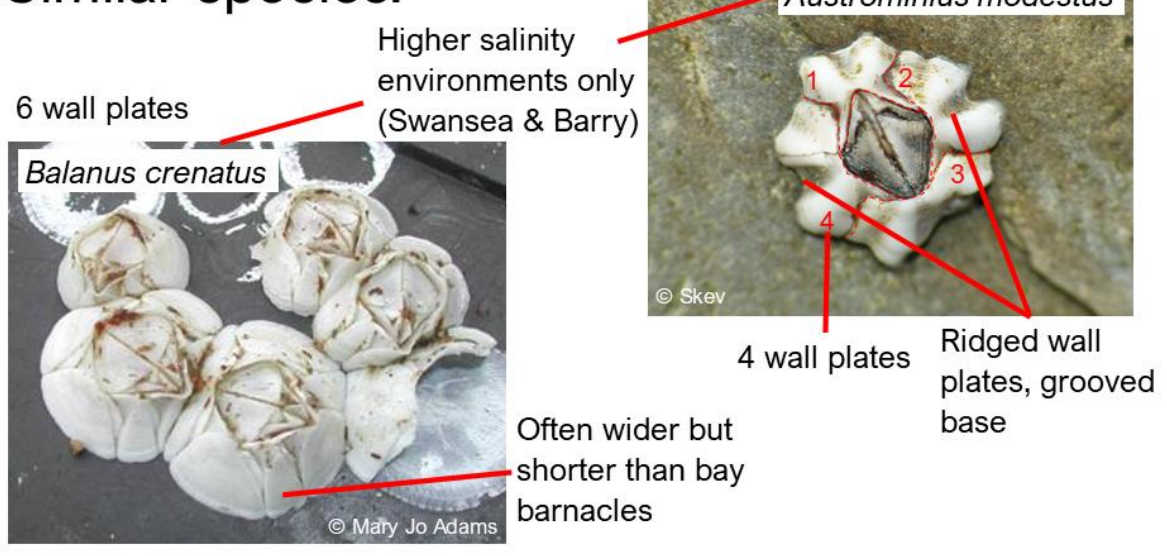

Known from: Newport, Cardiff

Environment: Brackish to low marine, subtidal or intertidal

UK distribution: Present on all coasts but restricted to estuaries

Size: Usually $\sim 10 \mathrm{~mm}$ diameter (max $23 \mathrm{~mm}$ )

Habitat: Hard surfaces: port walls, metal, lock gates etc.

Origin: Western Atlantic

Impacts: Fouling, competition, habitat modification 


\section{Austrominius modestus (Australasian barnacle)}

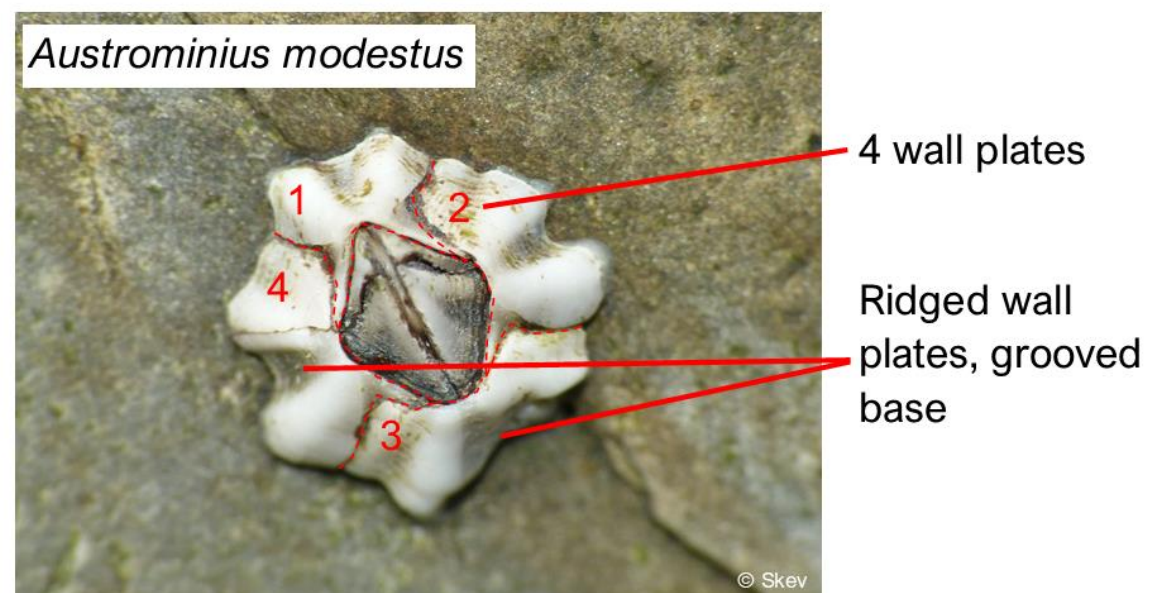

\section{Similar species:}

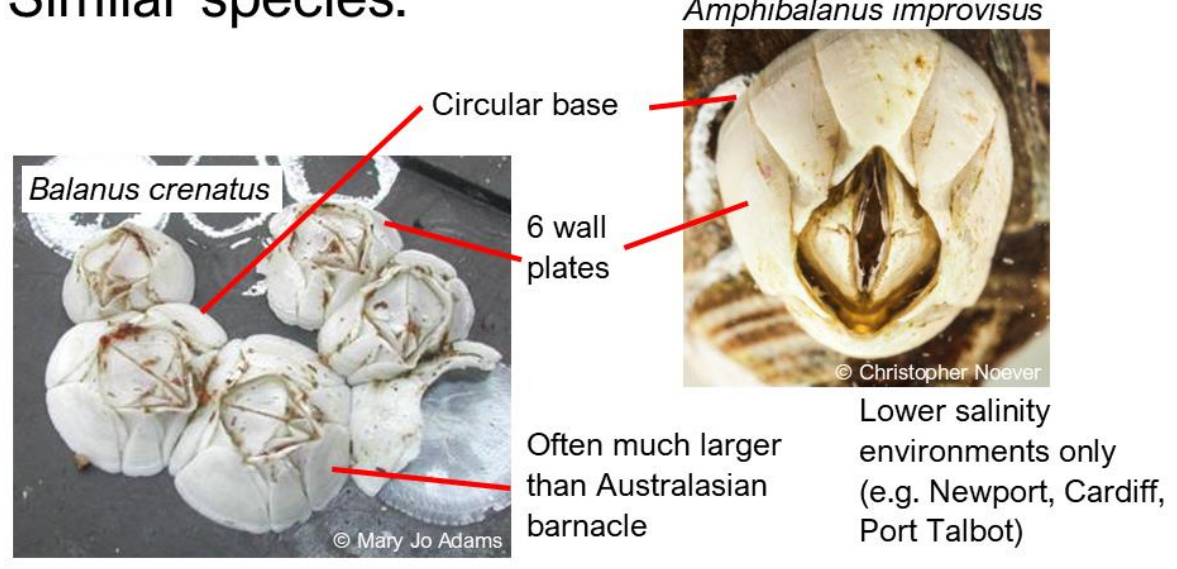

Known from: Barry, Swansea

Environment: Marine to high brackish, subtidal and intertidal

UK distribution: Widespread on all coasts

Size: up to $10 \mathrm{~mm}$ diameter

Habitat: Hard surfaces: port walls, metal, lock gates etc.

Origin: New Zealand

Impacts: Fouling, competition 


\section{Brachynotus sexdentatus (Mediterranean crab)}

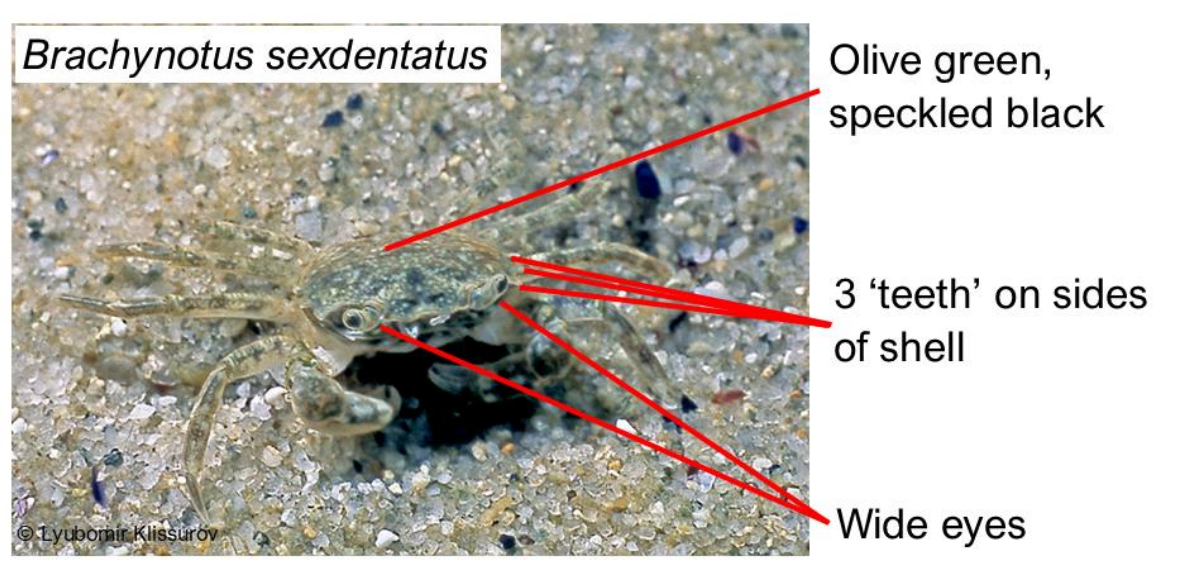

Similar species:

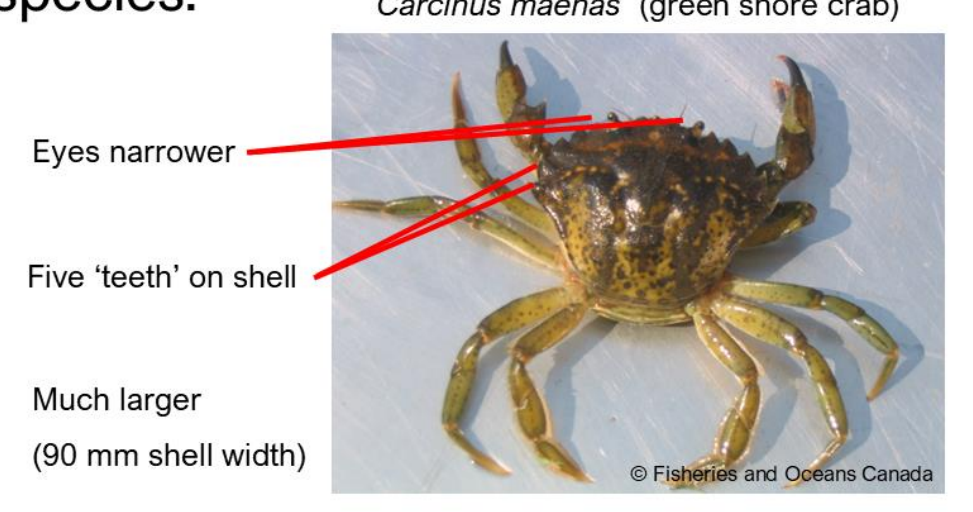

Known from: Swansea

Environment: Marine, initially introduced in artificially warmed water

UK distribution: Only ever found in the Port of Swansea

Size: Small; $<20 \mathrm{~mm}$ shell width

Habitat: Most materials but often hidden amongst other organisms

Origin: Mediterranean and Black Seas

Impacts: Largely unknown,

competition is likely 


\section{Bugula neritina (Brown bryozoan)}

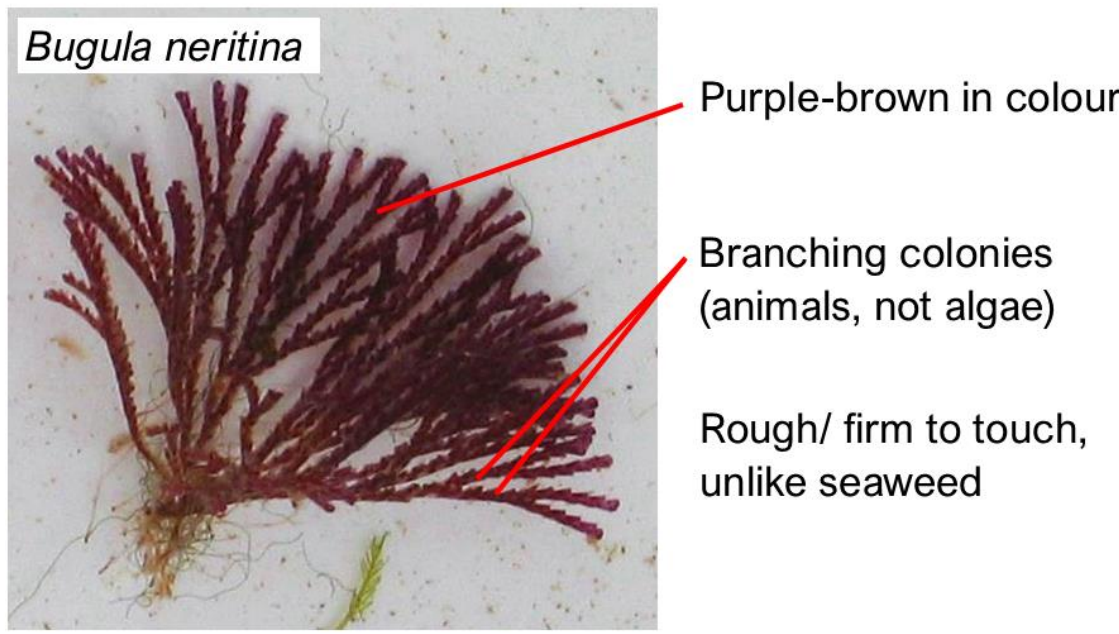

Similar species:

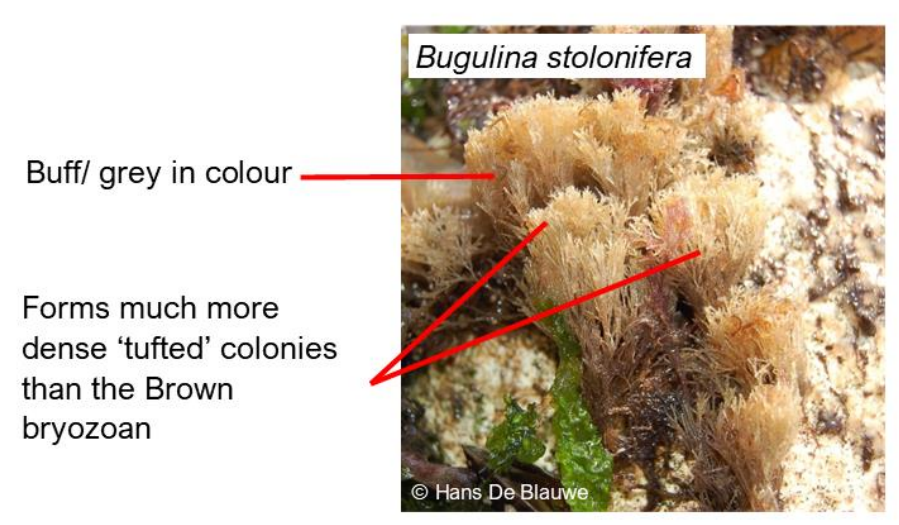

Known from: Swansea

Environment: Brackish and marine, intertidal and subtidal

UK distribution: Found on all coasts, predominantly in ports and marinas. Most common along south coast of England

Size: Colonies up to $10 \mathrm{~cm}$ tall

Habitat: Most port habitats (walls, rope, chains etc.)

Origin: Disputed

Impacts: Fouling 


\section{Bugulina stolonifera (A bryozoan; no official common name)}

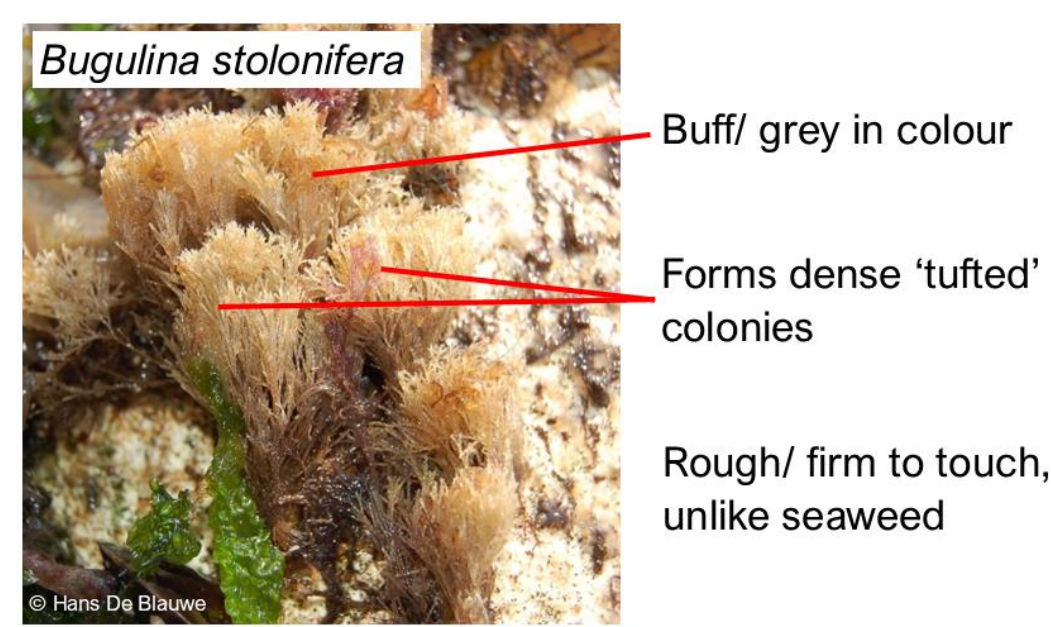

Similar species:

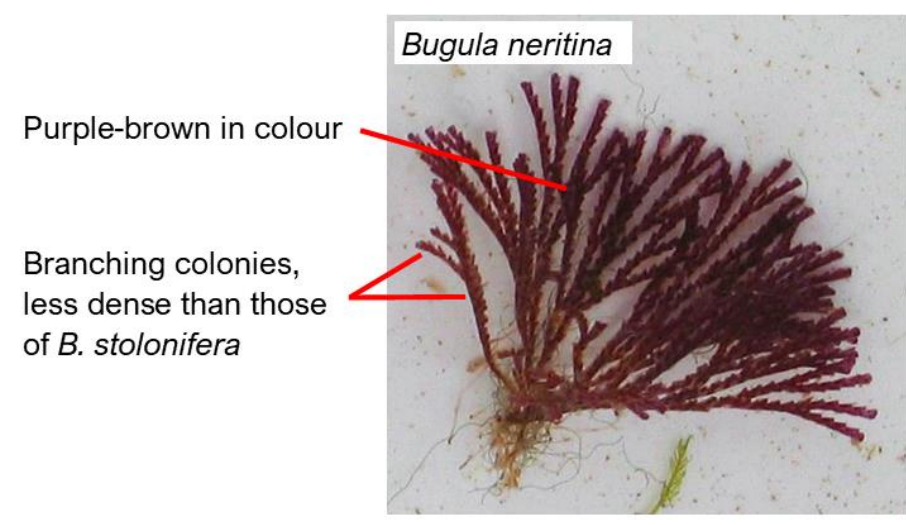

Known from: Barry, Swansea

Environment: Marine, subtidal to low intertidal

UK distribution: Present around the UK but most common on the south coast of England. Found in high abundance in South Wales ports

Size: Colonies up to approx. $7 \mathrm{~cm}$ tall Habitat: Most port habitats (walls, rope, chains etc.)

Origin: Disputed

Impacts: Fouling 


\section{Caprella mutica (Japanese skeleton shrimp)}

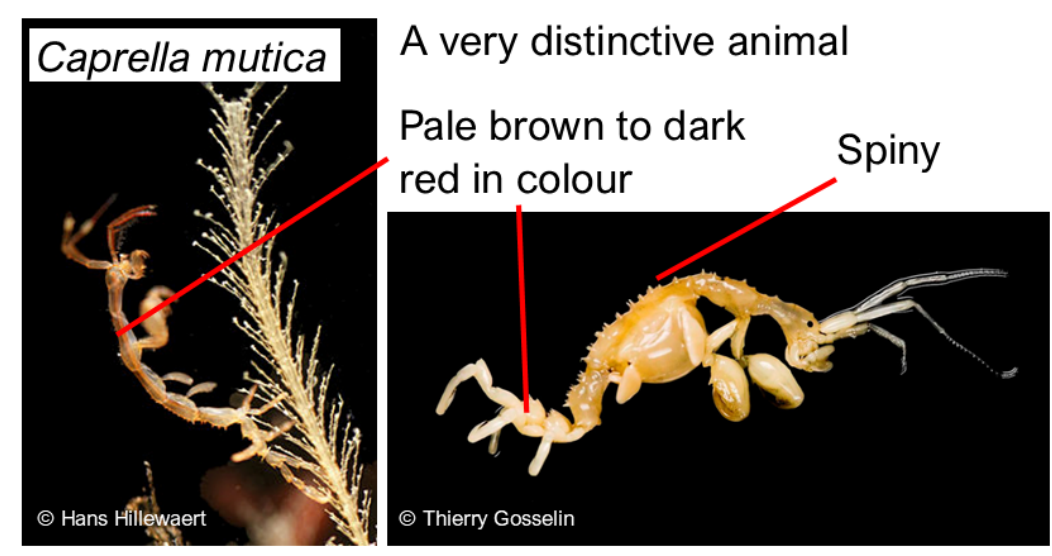

Similar species:

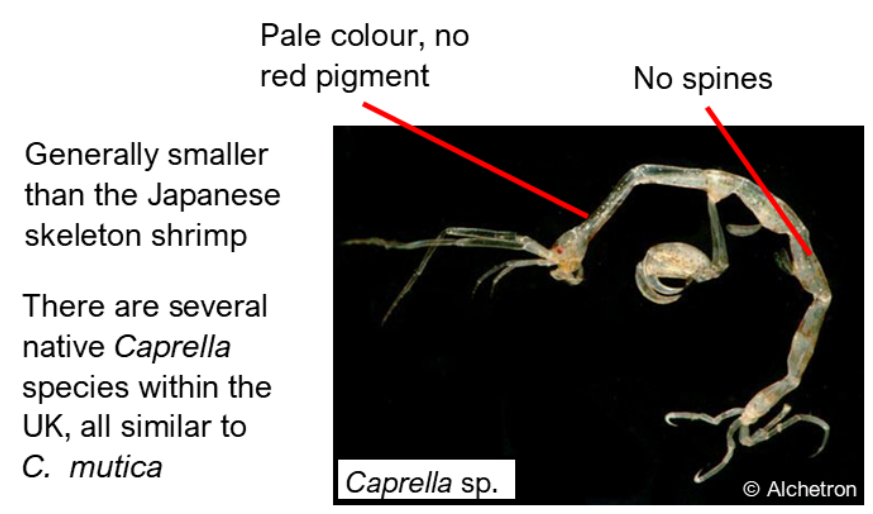

Known from: Barry, Swansea

Environment: Marine to mid-brackish, subtidal

UK distribution: Widespread, most common in Scotland and southern England

Size: Up to $50 \mathrm{~mm}$ in length

Habitat: Can be found in most habitats. Common on rope, often found alongside mussels.

Origin: North-west Asia, Sea of Japan Impacts: Competition, can form very dense aggregations 


\section{Cordylophora caspia (A hydroid, no common name)}

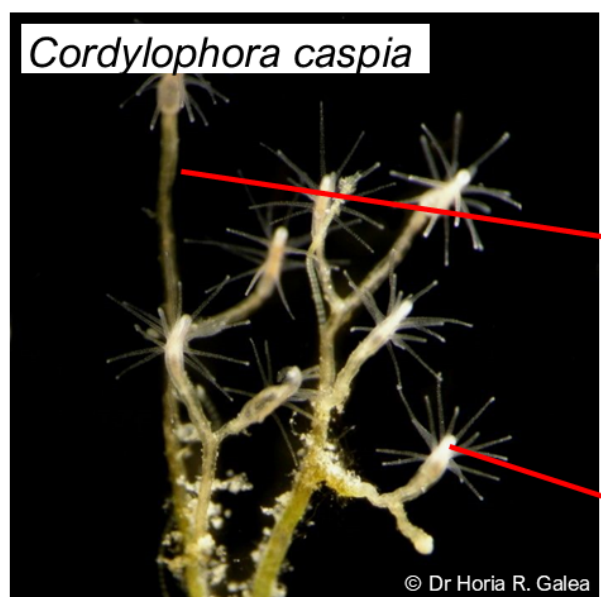

Similar species:

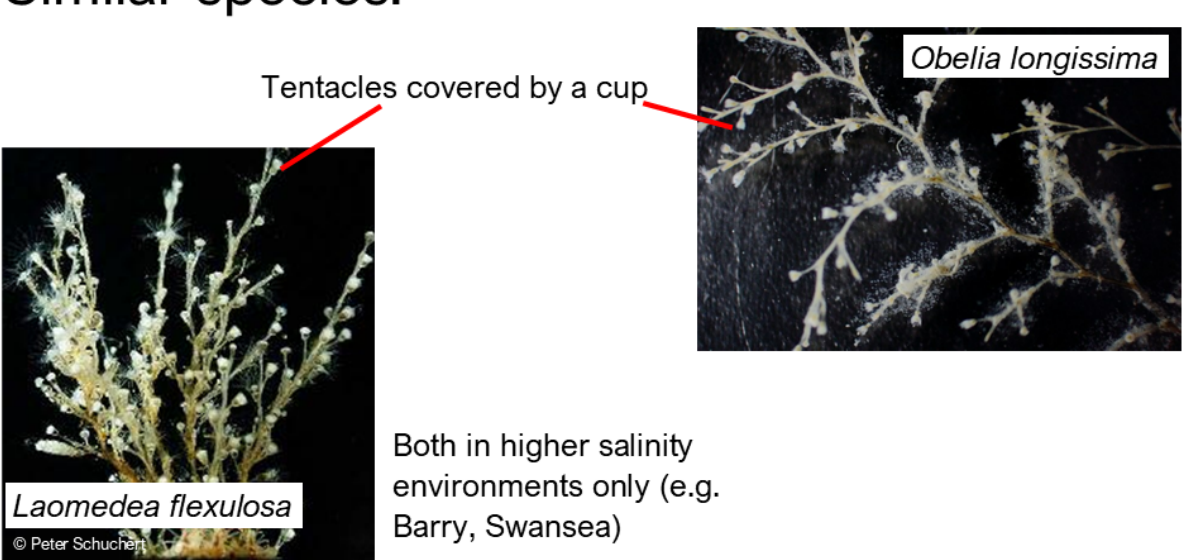

Unlikely to encounter or identify this species without using magnification

Very thin $(<1 \mathrm{~mm})$ 'stems'

Will lie flat out of the water and be erect in the water

Tentacles exposed with no covering (see below)

Barry, Swansea)
Known from: Cardiff, Port Talbot

Environment: Freshwater to low brackish, subtidal

UK distribution: Widespread in freshwater habitats. Most commonly reported in East Anglia.

Size: Up to $7 \mathrm{~cm}$ high

Habitat: Various habitats: walls, ropes, metal etc.

Origin: Black and Caspian Seas

Impacts: Fouling, competition 


\section{Diadumene lineata (Orange-striped anemone)}

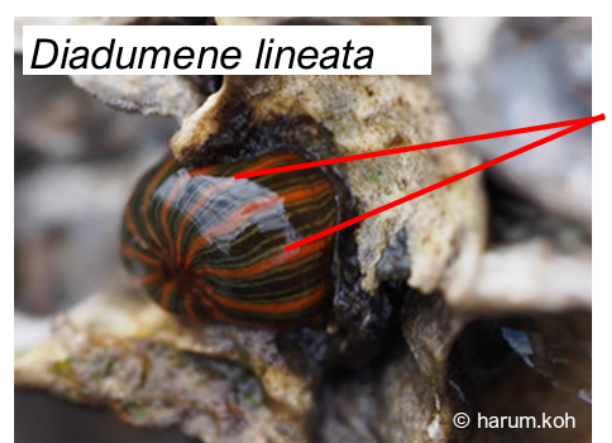

Distinctive colouring: green bands with orange strips

Can be intertidal, found on lock gates/ walls etc.

\section{Similar species:}

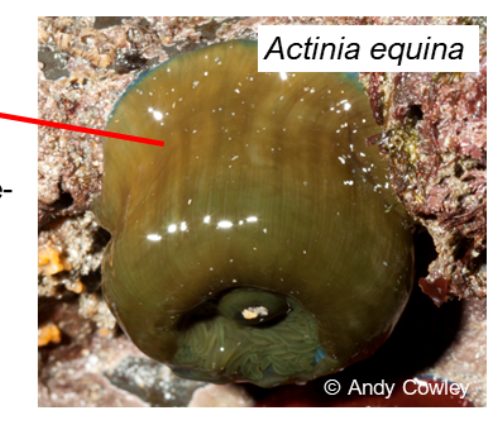

\section{Known from: Barry}

Environment: Brackish and marine, subtidal and intertidal

UK distribution: Widespread, most common along south coast of England

Size: Up to $20 \mathrm{~mm}$ diameter

Habitat: Hard surfaces: port walls, metal, lock gates etc.

Origin: Pacific coast of Asia

Impacts: Fouling, competition 


\section{Ficopomatus enigmaticus (Australian/Trumpet tube worm)}

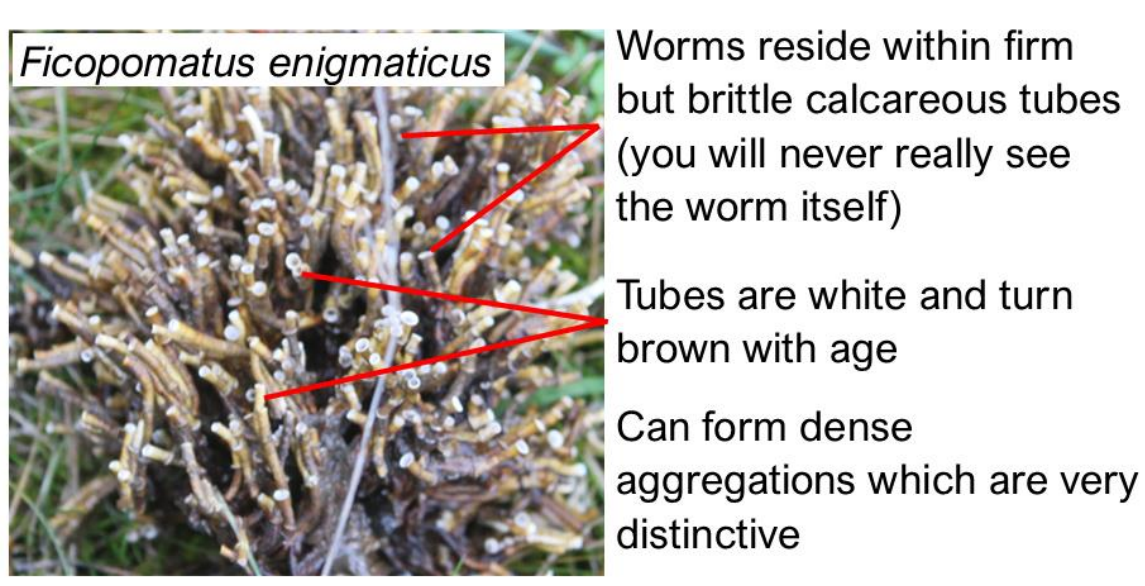

Similar species:

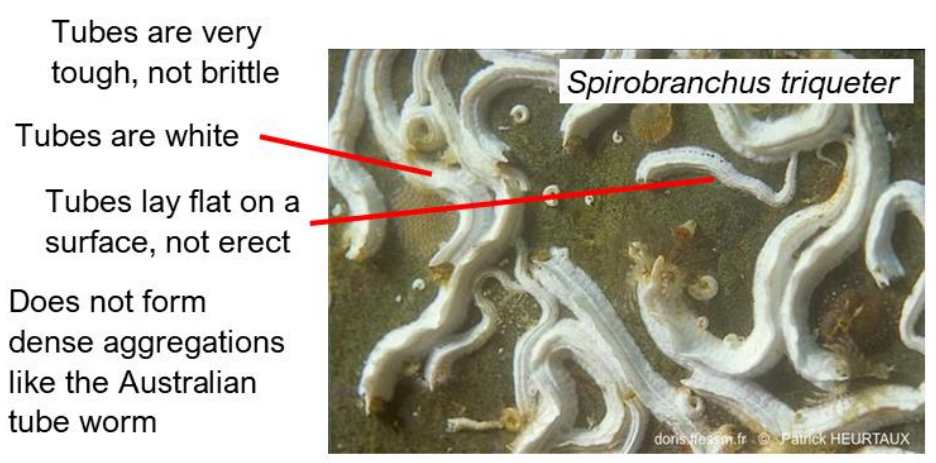

Known from: Newport, Cardiff, Barry

Environment: Brackish, subtidal

UK distribution: Widespread, most common in south Wales and southern England

Size: Tubes up to $10 \mathrm{~cm}$ in length

Habitat: Common on hard surfaces, also found on rope

Origin: Disputed

Impacts: Fouling, competition, habitat modification 


\section{Monocorophium sp. (An amphipod; no common name)}

Unlikely to encounter or identify these species without using magnification

Two known species of Monocorophium present in South Wales ports:

- M. acherusicum

- M. sextonae

There are no distinguishing features visible without magnification to tell the difference between the two

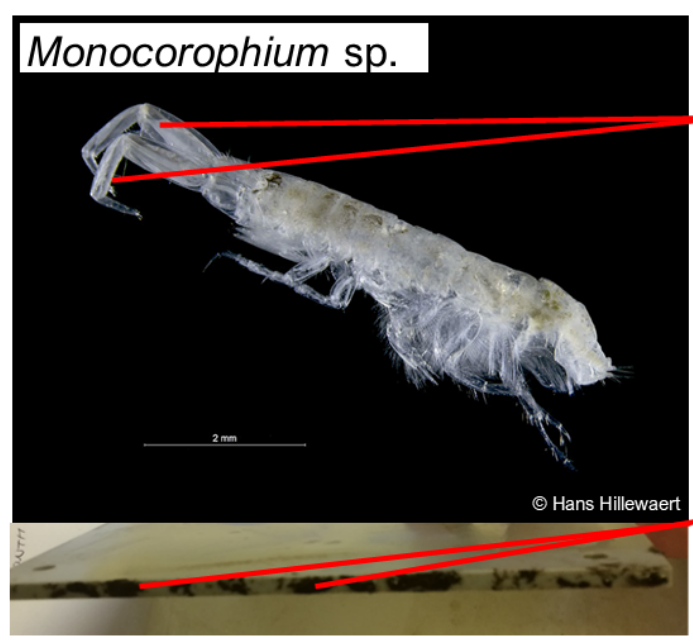

Large, thick second antennae

Generally lie flat when out of water

Often reside within clumps of mud and algae

Known from: Newport, Cardiff, Barry, Swansea

Environment: Brackish to marine, subtidal

UK distribution: Widespread, all coasts

Size: Small; $5 \mathrm{~mm}$ in length

Habitat: Potentially all surfaces, commonly in muddier/ silted areas

Origin: M. acherusicum - Disputed M. sextonae - New Zealand Impacts: Competition 


\section{Mytilopsis leucophaeata (Dark false mussel)}

Mytilopsis leucophaeata

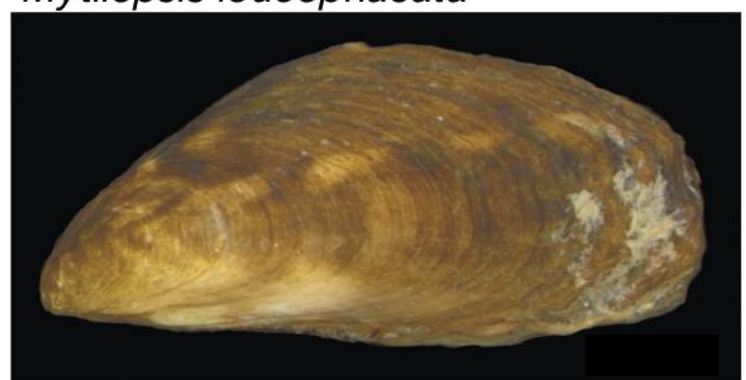

Distinctive size and

shape

No stripes

Low salinity habitats only (Port Talbot,

Cardiff)

Dark brown to black in colour

Similar species:

Common blue mussel

Adults much larger

than dark false mussel

Juveniles may be

similar size, shape and

colour to dark false

mussel but shells will

be weak and

translucent

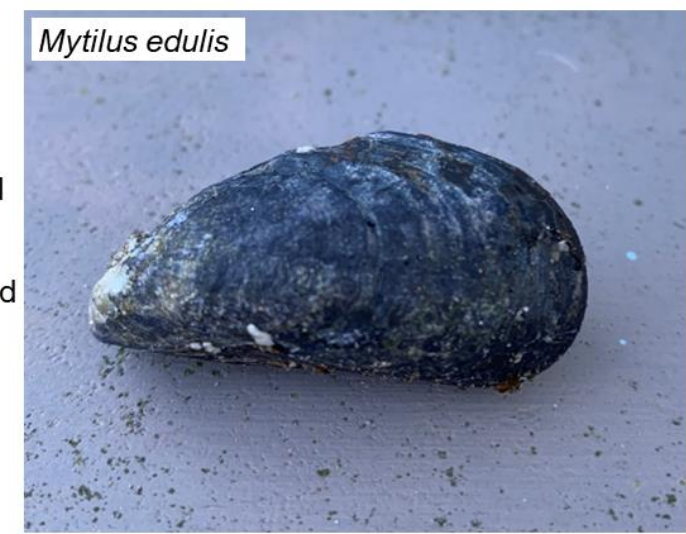

Known from: Port Talbot

Environment: Freshwater to low brackish, subtidal and intertidal

UK distribution: England and Wales, although not widespread. Can be found in isolated pools/ lakes and rivers

Size: Small; $<2 \mathrm{~cm}$ in length

Habitat: Most port habitats (walls, rope, chains etc.)

Origin: Gulf of Mexico

Impacts: Fouling, competition 


\section{Styela clava (Leathery sea squirt)}

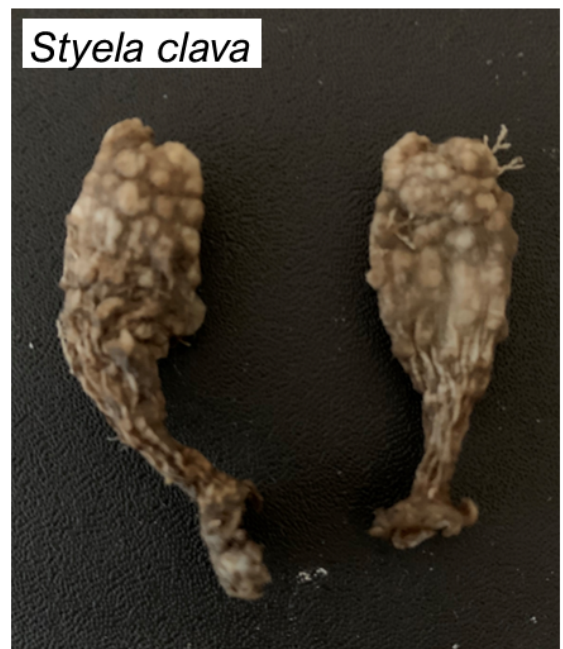

Similar species:

Can be similar in size to leathery sea squirt

Much softer, less rigid than leathery sea squirt but stil rough in texture

Translucent
Very tough, leathery to the touch with obvious bumps

‘Club’ shaped

Commonly reddishbrown in colour

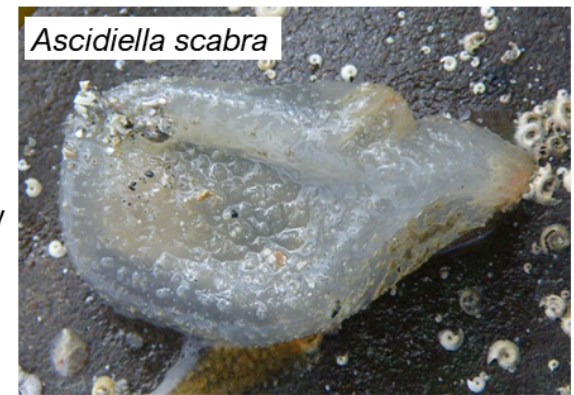

Known from: Barry, Swansea

Environment: Brackish to marine, subtidal and intertidal

UK distribution: Widespread, all coasts

Size: Up to $16 \mathrm{~cm}$ in length, usually more like $7-8 \mathrm{~cm}$

Habitat: Most port habitats (walls, rope, chains etc.)

Origin: Pacific coast of Asia

Impacts: Fouling, competition 


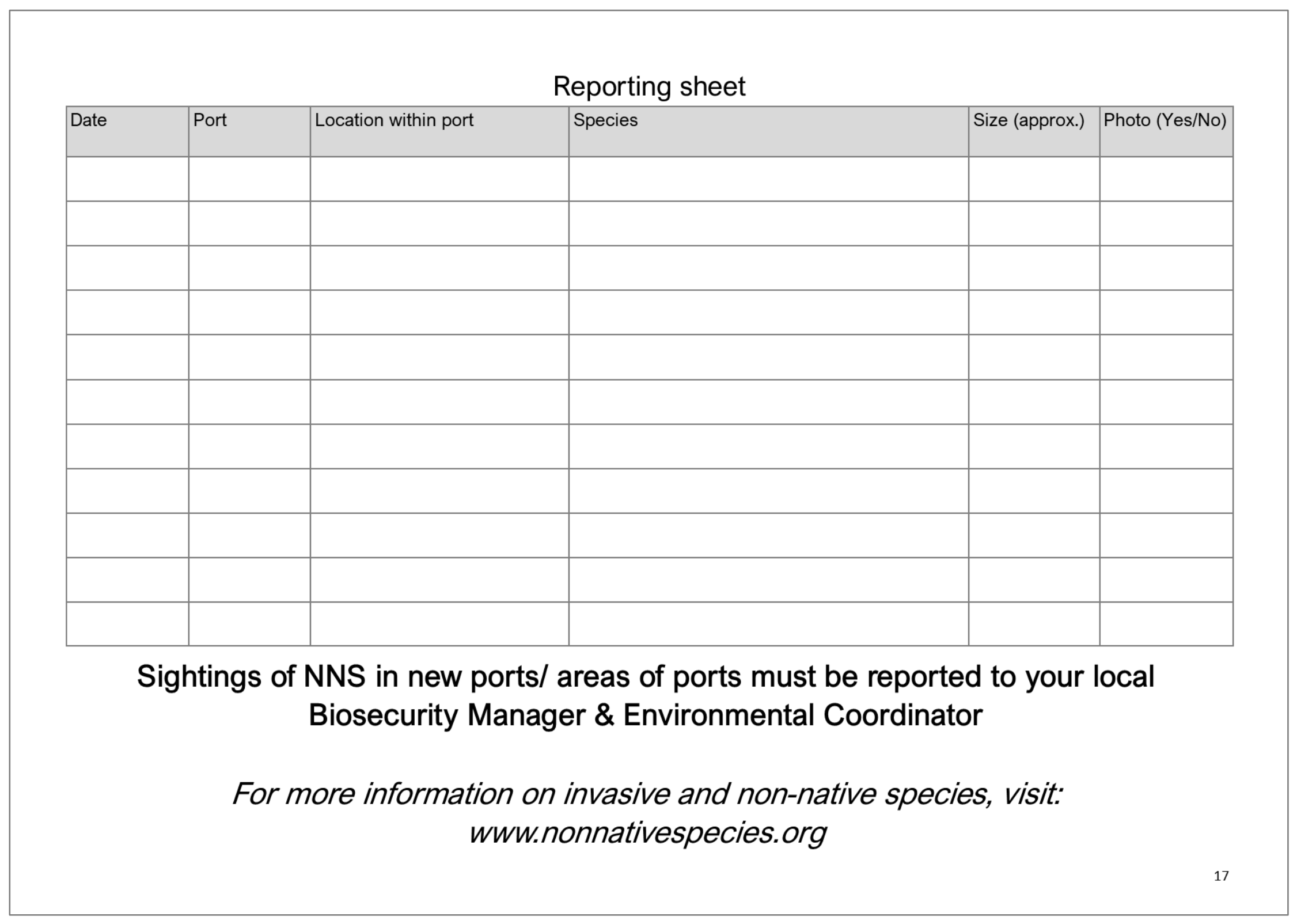




\section{REFERENCES}

Abt Associates Inc. 2008. Ecological and Economic Impacts and Invasion Management Strategies for the European Green Crab. [Online] Available from: https://www.epa.gov/environmental-economics/ecological-andeconomic-impacts-and-invasion-management-strategies-european

Agamuthu, P., Mehran, S.B., Norkhairah, A. and Norkhairiyah, A. 2019. Marine debris: A review of impacts and global initiatives. Waste Management and Research. 37(10), pp.987-1002.

Aikins, S. and Kikuchi, E. 2001. Studies on habitat selection by amphipods using artificial substrates within an estuarine environment. Hydrobiologia. 457, pp.77-86.

Anderson, L.W.J. 2005. California's reaction to Caulerpa taxifolia: A model for invasive species rapid response. Biological Invasions. 7(6), pp.1003-1016.

Anderson, L.W.J. 2007. Control of invasive seaweeds. Botanica Marina. 50(5-6), pp.418-437.

Anderson, M., Gorley, R. and Clarke, K. 2008. PERMANOVA+ for PRIMER. Guide to software and statistical methods. Plymouth, UK: PRIMER-E.

Andrady, A.L. 2011. Microplastics in the marine environment. Marine Pollution Bulletin. 62(8), pp.1596-1605.

Arenas, F., Bishop, J.D.D., Carlton, J.T., Dyrynda, P.J., Farnham, W.F., Gonzalez, D.J., Jacobs, M.W., Lambert, C., Lambert, G., Nielsen, S.E., Pederson, J.A., Porter, J.S., Ward, S. and Wood, C.A. 2006. Alien species and other notable records from a rapid assessment survey of marinas on the south coast of England. Journal of the Marine Biological Association of the United Kingdom. 86(6), pp.1329-1337.

Ashton, G. V., Willis, K.J., Burrows, M.T. and Cook, E.J. 2007. Environmental tolerance of Caprella mutica: Implications for its distribution as a marine nonnative species. Marine Environmental Research. 64(3), pp.305-312. 
Ashton, G. V., Willis, K.J., Cook, E.J. and Burrows, M. 2007. Distribution of the introduced amphipod, Caprella mutica Schurin, 1935 (Amphipoda: Caprellida: Caprellidae) on the west coast of Scotland and a review of its global distribution. Hydrobiologia. 590(1), pp.31-41.

Associated British Ports, 2020. Locations. [Online] [Accessed 16 June 2020] Available from: https://www.abports.co.uk/

Astudillo, J.C., Bravo, M., Dumont, C.P. and Thiel, M. 2009. Detached aquaculture buoys in the SE Pacific: Potential dispersal vehicles for associated organisms. Aquatic Biology. 5(3), pp.219-231.

Au, S.Y., Bruce, T.F., Bridges, W.C. and Klaine, S.J. 2015. Responses of Hyalella azteca to acute and chronic microplastic exposures. Environmental Toxicology and Chemistry. 34(11), pp.2564-2572.

Aubet, M.E. 2001. The Phoenicians and the West: politics, colonies and trade. Cambridge: Cambridge University Press.

Avio, C.G., Gorbi, S. and Regoli, F. 2017. Plastics and microplastics in the oceans: From emerging pollutants to emerged threat. Marine Environmental Research. 128, pp.2-11.

Azmi, F., Primo, C., Hewitt, C.L. and Campbell, M.L. 2015. Assessing marine biosecurity risks when data are limited: bioregion pathway and species-based exposure analyses. ICES Journal of Marine Science. 72(3), pp.1078-1091.

Bailey, S.A. 2015. An overview of thirty years of research on ballast water as a vector for aquatic invasive species to freshwater and marine environments. Aquatic Ecosystem Health and Management. 18(3), pp.261-268.

Balbi, T., Franzellitti, S., Fabbri, R., Montagna, M., Fabbri, E. and Canesi, L. 2016. Impact of bisphenol A (BPA) on early embryo development in the marine mussel Mytilus galloprovincialis: Effects on gene transcription. Environmental Pollution. 218, pp.996-1004.

Bangor University 2015. Wales Marine Non-native Species Inshore Monitoring Network. 
Bax, N., Williamson, A., Aguero, M., Gonzalez, E. and Geeves, W. 2003. Marine invasive alien species: A threat to global biodiversity. Marine Policy. 27(4), pp.313-323.

Beaumont, J.C., Brown, C.J. and Sayer, M.D.J. 2007. Evaluation of techniques used in the assessment of subtidal epibiotic assemblage structure. Biofouling. 23(5), pp.343-356.

Bellard, C., Cassey, P. and Blackburn, T.M. 2016. Alien species as a driver of recent extinctions. Biology Letters. 12(4).

Benton, T.G., Solan, M., Travis, J.M.J. and Sait, S.M. 2007. Microcosm experiments can inform global ecological problems. Trends in Ecology and Evolution. 22(10), pp.516-521.

Berntsson, K. and Jonsson, P. 2003. Temporal and Spatial Patterns in Recruitment and Succession of a Temperate Marine Fouling Assemblage: a Comparison of Static Panels and Boat Hulls during the Boating Season. Biofouling. 19(3), pp.187-195.

Berry, K.J., Mielke, P.W. and Mielke, H.W. 2002. The Fisher-Pitman permutation test: An attractive alternative to the F test. Psychological Reports. 90, pp.495502.

Bishop, J.D.D., Wood, C.A., Yunnie, A.L.E. and Griffiths, C.A. 2015. Unheralded arrivals: Non-native sessile invertebrates in marinas on the English coast. Aquatic Invasions. 10(3), pp.249-264.

Blackburn, T.M., Essl, F., Evans, T., Hulme, P.E., Jeschke, J.M., Kühn, I., Kumschick, S., Marková, Z., Mrugała, A., Nentwig, W., Pergl, J., Pyšek, P., Rabitsch, W., Ricciardi, A., Richardson, D.M., Sendek, A., Vilà, M., Wilson, J.R.U., Winter, M., Genovesi, P. and Bacher, S. 2014. A Unified Classification of Alien Species Based on the Magnitude of their Environmental Impacts. PLoS Biology. 12(5).

Blackburn, T.M., Pyšek, P., Bacher, S., Carlton, J.T., Duncan, R.P., Jarošík, V., Wilson, J.R.U. and Richardson, D.M. 2011. A proposed unified framework for biological invasions. Trends in Ecology and Evolution. 26(7), pp.333-339. 
Blockley, D.J. and Chapman, M.G. 2006. Recruitment determines differences between assemblages on shaded or unshaded seawalls. Marine Ecology Progress Series. 327(December), pp.27-36.

Bo, M., Bava, S., Canese, S., Angiolillo, M., Cattaneo-Vietti, R. and Bavestrello, G. 2014. Fishing impact on deep Mediterranean rocky habitats as revealed by ROV investigation. Biological Conservation. 171, pp.167-176.

Boon, P.J., Clarke, S.A. and Copp, G.H. 2020. Alien species and the EU Water Framework Directive: a comparative assessment of European approaches. Biological Invasions. 22(4), pp.1497-1512.

Booy, O., Mill, A.C., Roy, H.E., Hiley, A., Moore, N., Robertson, P., Baker, S., Brazier, M., Bue, M., Bullock, R., Campbell, S., Eyre, D., Foster, J., HattonEllis, M., Long, J., Macadam, C., Morrison-Bell, C., Mumford, J., Newman, J., Parrott, D., Payne, R., Renals, T., Rodgers, E., Spencer, M., Stebbing, P., Sutton-Croft, M., Walker, K.J., Ward, A., Whittaker, S. and Wyn, G. 2017. Risk management to prioritise the eradication of new and emerging invasive non-native species. Biological Invasions. 19(8), pp.2401-2417.

Borrell, Y.J., Miralles, L., Do Huu, H., Mohammed-Geba, K. and Garcia-Vazquez, E. 2017. DNA in a bottle - Rapid metabarcoding survey for early alerts of invasive species in ports. PLoS ONE. 12(9), pp.1-17.

Boudouresque, C.F. and Verlaque, M. 2002. Biological pollution in the Mediterranean Sea: invasive versus introduced macrophytes. Marine Pollution Bulletin. 44, pp.32-38.

Bracewell, S.A., Spencer, M., Marrs, R.H., Iles, M. and Robinson, L.A. 2012. Cleft, Crevice, or the Inner Thigh: 'Another Place' for the Establishment of the Invasive Barnacle Austrominius modestus (Darwin, 1854). PLoS ONE. 7(11).

Bringer, A., Thomas, H., Prunier, G., Dubillot, E., Bossut, N., Churlaud, C., Cléreandeau, C., Le Bihanic, F. and Cachot, J. 2020. High density polyethylene (HDPE) microplastics impair development and swimming activity of Pacific oyster D-larvae, Crassostrea gigas, depending on particle size. Environmental Pollution. 260, 113978. 
Brown, N.E.M., Milazzo, M., Rastrick, S.P.S., Hall-Spencer, J.M., Therriault, T.W. and Harley, C.D.G. 2018. Natural acidification changes the timing and rate of succession, alters community structure, and increases homogeneity in marine biofouling communities. Global Change Biology. 24(1), pp.1-38.

Bruschetti, M., Luppi, T., Fanjul, E., Rosenthal, A. and Iribarne, O. 2008. Grazing effect of the invasive reef-forming polychaete Ficopomatus enigmaticus (Fauvel) on phytoplankton biomass in a SW Atlantic coastal lagoon. Journal of Experimental Marine Biology and Ecology. 354(2), pp.212-219.

Buscher, E., Mathews, D.L., Bryce, C., Bryce, K., Joseph, D. and Ban, N.C. 2020. Applying a Low Cost, Mini Remotely Operated Vehicle (ROV) to Assess an Ecological Baseline of an Indigenous Seascape in Canada. Frontiers in Marine Science. 7, pp.1-12.

Byrnes, J.E., Reynolds, P.L. and Stachowicz, J.J. 2007. Invasions and extinctions reshape coastal marine food webs. PLoS ONE. 2(3), pp.1-7.

Callow, J.A. and Callow, M.E. 2011. Trends in the development of environmentally friendly fouling-resistant marine coatings. Nature Communications. 2:244.

Campbell, M.L. 2011. Assessing biosecurity risk associated with the importation of non-indigenous microalgae. Environmental Research. 111(7), pp.989-998.

Canning-Clode, J., Fofonoff, P., McCann, L., Carlton, J.T. and Ruiz, G. 2013.

Marine invasions on a subtropical island: Fouling studies and new records in a recent marina on Madeira island (Eastern Atlantic Ocean). Aquatic Invasions. 8(3), pp.261-270.

Cánovas-Molina, A., Montefalcone, M., Bavestrello, G., Cau, A., Bianchi, C.N., Morri, C., Canese, S. and Bo, M. 2016. A new ecological index for the status of mesophotic megabenthic assemblages in the mediterranean based on ROV photography and video footage. Continental Shelf Research. 121, pp.13-20.

Carlton, J.T. 1996. Pattern, process, and prediction in marine invasion ecology. Biological Conservation. 78(1-2), pp.97-106. 
Carlton, J.T. 1985. Transoceanic and interoceanic dispersal of coastal marine organisms: the biology of ballast water In: M. Barnes, ed. Oceanography and Marine Biology. An Annual Review. Aberdeen, Scotland, UK: Aberdeen University Press, pp.313-371.

Carlton, J.T. and Geller, J.B. 1993. Ecological Roulette: The global transport of nonindigenous marine organisms. Science. 261(5117), pp.78-82.

Carlton, J.T. and Hodder, J. 1995. Biogeography and dispersal of coastal marine organisms: experimental studies on a replica of a 16th-century sailing vessel. Marine Biology. 121(4), pp.721-730.

Carlton, J.T., Chapman, J.W., Geller, J.B., Miller, J.A., Carlton, D.A., McCuller, M.I., Treneman, N.C., Steves, B.P. and Ruiz, G.M. 2017. Tsunami-driven rafting: Transoceanic species dispersal and implications for marine biogeography. Science. 357, pp. 1402-1406.

Charles, M., Faillettaz, R., Desroy, N., Fournier, J. and Costil, K. 2018. Distribution, associated species and extent of biofouling "reefs" formed by the alien species Ficopomatus enigmaticus (Annelida, Polychaeta) in marinas. Estuarine, Coastal and Shelf Science. 212, pp.164-175.

Cifuentes, M., Krueger, I., Dumont, C.P., Lenz, M. and Thiel, M. 2010. Does primary colonization or community structure determine the succession of fouling communities? Journal of Experimental Marine Biology and Ecology. 395, pp.10-20.

Clare, D.S., Spencer, M., Robinson, L.A. and Frid, C.L.J. 2016. Species-specific effects on ecosystem functioning can be altered by interspecific interactions. PLoS ONE. 11(11), pp.1-17.

Clarke Murray, C., Pakhomov, E.A. and Therriault, T.W. 2011. Recreational boating: A large unregulated vector transporting marine invasive species. Diversity and Distributions. 17(6), pp.1161-1172.

Cognetti, G. and Maltagliati, F. 2000. Biodiversity and adaptive mechanisms in brackish water fauna. Marine Pollution Bulletin. 40(1), pp.7-14. 
Cohen, A.N., Harris, L.H., Bingham, B.L., Carlton, J.T., Chapman, J.W., Lambert, C.C., Lambert, G., Ljubenkov, J.C., Murray, S.N., Rao, L.C., Reardon, K. and Schwindt, E. 2005. Rapid Assessment Survey for exotic organisms in southern California bays and harbors, and abundance in port and non-port areas. Biological Invasions. 7(6), pp.995-1002.

Colautti, R.I. and MacIsaac, H.J. 2004. A neutral terminology to define 'invasive' species. Diversity and Distributions. 10, pp.135-141.

Cole, M., Lindeque, P., Halsband, C. and Galloway, T.S. 2011. Microplastics as contaminants in the marine environment: A review. Marine Pollution Bulletin. 62(12), pp.2588-2597.

Collin, S.B., Tweddle, J.F. and Shucksmith, R.J. 2015. Rapid assessment of marine non-native species in the Shetland Islands, Scotland. BioInvasions Records. 4(3), pp.147-155.

Commission Regulation (EC) No. 535/2008 Laying Down Detailed Rules for the implementation of Council Regulation (EC) No 708/2007 Concerning Use of Alien and Locally Absent Species in Aquaculture. [Online] [Accessed 20 May 2020] Available from: http://eur-lex.europa.eu/

Connell, S.D. 2000. Floating pontoons create novel habitats for subtidal epibiota. Journal of Experimental Marine Biology and Ecology. 247(2), pp.183-194.

Cook, E.J., Beveridge, C., Twigg, G. \& Macleod, A. 2015. Assessing the effectiveness of early warning systems for the detection of marine invasive non-native species in Scottish waters. Scottish Natural Heritage Commissioned Report No. 874.

Cook, E.J., Willis, K.J. and Lozano-Fernandez, M. 2007. Survivorship, growth and reproduction of the non-native Caprella mutica Schurin, 1935 (Crustacea: Amphipoda). Hydrobiologia. 590(1), pp.55-64.

Costello, M.J., Claus, S., Dekeyzer, S., Vandepitte, L., Tuama, É., Lear, D. and Tyler-Walters, H. 2015. Biological and ecological traits of marine species. PeerJ. 3:e1201, pp.1-29. 
Council Directive 2000/60/EC of the European Parliament and of the Council of 23 October 2000 establishing a framework for Community action in the field of water policy. [Online]. [Accessed 13 May 2020] Available from: https://eurlex.europa.eu/

Council Directive 2008/56/EC of the European Parliament and of the Council of 17 June 2008 establishing a framework for community action in the field of marine environmental policy (Marine Strategy Framework Directive).

[Online]. [Accessed 13 May 2020] Available from: https://eur-lex.europa.eu/ Council Regulation (EC) No. 708/2007 of 11 June 2007 concerning use of alien and locally absent species in aquaculture. [Online] [Accessed 20 May 2020] Available from: http://eur-lex.europa.eu/

Courtois, P., Figuieres, C., Mulier, C. and Weill, J. 2018. A Cost-Benefit Approach for Prioritizing Invasive Species. Ecological Economics. 146, pp.607-620.

Coutts, A.D.M. and Forrest, B.M. 2007. Development and application of tools for incursion response: Lessons learned from the management of the fouling pest Didemnum vexillum. Journal of Experimental Marine Biology and Ecology. 342, pp.154-162.

Coutts, A.D.M., Piola, R.F., Hewitt, C.L., Connell, S.D. and Gardner, J.P.A. 2010. Effect of vessel voyage speed on survival of biofouling organisms: Implications for translocation of non-indigenous marine species. Biofouling. 26(1), pp.1-13.

Critchley, A.T., Farnham, W.F. and Morrell, S.L. 1983. A Chronology of New European Sites of Attachment for the Invasive Brown Alga, Sargassum Muticum, 1973-1981. Journal of the Marine Biological Association of the United Kingdom. 63, pp.799-811.

Crooks, J.A. 2005. Lag times and exotic species: The ecology and management of biological invasions in slow-motion. Ecoscience. 12(3), pp.316-329.

Crooks, J.A. 2009. The Role of Exotic Marine Ecosystem Engineers In: G. Rilov and J. A. Crooks, eds. Biological Invasions in Marine Ecosystems. Berlin Heidelberg: Springe-Verlag, pp.287-304. 
Crooks, J.A., Chang, A.L. and Ruiz, G.M. 2011. Aquatic pollution increases the relative success of invasive species. Biological Invasions. 13(1), pp.165-176.

Cuesta, J.A., Schubart, C.D. and Rodriguez, A. 2000. Larval development of Brachynotus sexdentatus (Risso, 1827) (Decapoda, Brachyura) reared under laboratory conditions, with notes on larval characters of the Varunidae. Invertebrate Reproduction \& Development. 38(3), pp.207-223.

Dafforn, K.A., Johnston, E.L. and Glasby, T.M. 2009. Shallow moving structures promote marine invader dominance. Biofouling. 25(3), pp.277-287.

Dahlstrom, A., Hewitt, C.L. and Campbell, M.L. 2011. A review of international, regional and national biosecurity risk assessment frameworks. Marine Policy. 35(2), pp.208-217.

Davidson, I.C., Brown, C.W., Sytsma, M.D. and Ruiz, G.M. 2009. The role of containerships as transfer mechanisms of marine biofouling species. Biofouling. 25(7), pp.645-655.

Davidson, I.C., Zabin, C.J., Chang, A.L., Brown, C.W., Sytsma, M.D. and Ruiz, G.M. 2010. Recreational boats as potential vectors of marine organisms at an invasion hotspot. Aquatic Biology. 11(2), pp.179-191.

DEFRA 2011. Biodiversity 2020: A strategy for England's wildlife and ecosystem services. [Online] Available from: https://www.gov.uk/government/publications/biodiversity-2020-a-strategy-forengland-s-wildlife-and-ecosystem-services

Delaney, D.G., Edwards, P.K. and Leung, B. 2012. Predicting regional spread of non-native species using oceanographic models: Validation and identification of gaps. Marine Biology. 159(2), pp.269-282.

Devin, S. and Beisel, J.N. 2007. Biological and ecological characteristics of invasive species: A gammarid study. Biological Invasions. 9(1), pp.13-24. 
Di Poi, E., Kraus, R., Cabrini, M., Finotto, S., Flander-Putrle, V., Grego, M., Kužat, N., Ninčević Gladan, Ž., Pezzolesi, L., Riccardi, E., Bernardi Aubry, F. and Bastianini, M. 2019. Dinoflagellate resting cysts from surface sediments of the Adriatic Ports: Distribution and potential spreading patterns. Marine Pollution Bulletin. 147, pp.185-208.

Dineen Jr., J.F. and Hines, A.H. 1992. Interactive effects of salinity and adult extract upon settlement of the estuarine barnacle Balanus improvisus (Darwin, 1854). Journal of Experimental Marine Biology and Ecology. 156(2), pp.239-252.

Dobretsov, S. V., Qian, P.Y. and Wahl, M. 2005. Effect of solar ultraviolet radiation on the formation of shallow, early successional biofouling communities in Hong Kong. Marine Ecology Progress Series. 290, pp.55-65.

Duffy, J.E. 2009. Why biodiversity is important to the functioning of real-world ecosystems. Frontiers in Ecology and the Environment. 7(8), pp.437-444.

Dupont, L., Viard, F., Davis, M.H., Nishikawa, T. and Bishop, J.D.D. 2010. Pathways of spread of the introduced ascidian Styela clava (Tunicata) in Northern Europe, as revealed by microsatellite markers. Biological Invasions. 12(8), pp.2707-2721.

EEA 2007. Halting the loss of biodiversity by 2010: proposal for a first set of indicators to monitor progress in Europe [Online]. Available from: http://www.eea.europa.eu/publications/technical_report_2007_11/.

EEA, 2012. The impacts of invasive alien species in Europe, EEA Technical Report No. 16/2012. [Online] [Accessed 28 September 2020] Available from: https://www.eea.europa.eu/publications/impacts-of-invasive-alien-species

Endresen, Ø., Lee Behrens, H., Brynestad, S., Bjørn Andersen, A. and Skjong, R. 2004. Challenges in global ballast water management. Marine Pollution Bulletin. 48(7-8), pp.615-623.

Eno, N.C., Robin, A.C. and Sanderson, W.G. 1997. Non-native marine species in British waters: a review and directory. Peterborough: Joint Nature Conservation Committee, JNCC. 
Environment (Wales) Act 2016. [Online] [Accessed 20 December 2019] Available from: http://www.legislation.gov.uk/

Epstein, G., Hawkins, S.J. and Smale, D.A. 2018. Removal treatments alter the recruitment dynamics of a global marine invader - Implications for management feasibility. Marine Environmental Research. 140, pp.322-331.

Evans, A.J., Firth, L.B., Hawkins, S.J., Hall, A.E., Ironside, J.E., Thompson, R.C. and Moore, P.J. 2019. From ocean sprawl to blue-green infrastructure - A UK perspective on an issue of global significance. Environmental Science and Policy. 91, pp.60-69.

Ficetola, G.F., Miaud, C., Pompanon, F. and Taberlet, P. 2008. Species detection using environmental DNA from water samples. Biology Letters. 4, pp.423-425.

Firestone, J. and Corbett, J.J. 2005. Coastal and port environments: International legal and policy responses to reduce Ballast Water introductions of potentially invasive species. Ocean Development and International Law. 36(3), pp.291316.

Fitridge, I., Dempster, T., Guenther, J. and de Nys, R. 2012. The impact and control of biofouling in marine aquaculture: A review. Biofouling. 28(7), pp.649-669.

Floerl, O. and Coutts, A. 2009. Potential ramifications of the global economic crisis on human-mediated dispersal of marine non-indigenous species. Marine Pollution Bulletin. 58(11), pp.1595-1598.

Floerl, O., Inglis, G.J., Dey, K. and Smith, A. 2009. The importance of transport hubs in stepping-stone invasions. Journal of Applied Ecology. 46(1), pp.37-45.

Floerl, O., Inglis, G.J., Peacock, L. and Plew, D. 2012. The Efficacy of Settlement Plate Arrays for Marine Surveillance. MPI Technical Paper No: 2012/16.

Folino-Rorem, N.C. and Indelicato, J. 2005. Controlling biofouling caused by the colonial hydroid Cordylophora caspia. Water Research. 39(12), pp.27312737.

Foster, V., Giesler, R.J., Wilson, A.M.W., Nall, C.R. and Cook, E.J. 2016. Identifying the physical features of marina infrastructure associated with the presence of non-native species in the UK. Marine Biology. 163(8), pp.1-14. 
Fridley, J.D., Stachowicz, J.J., Naeem, S., Sax, D.F., Seabloom, E.W., Smith, M.D., Stohlgren, T.J., Tilman, D. and Von Holle, B. 2007. The invasion paradox: Reconciling pattern and process in species invasions. Ecology. 88(1), pp.3-17.

Galil, B.S., Marchini, A., Occhipinti-Ambrogi, A., Minchin, D., Narščius, A., Ojaveer, H. and Olenin, S. 2014. International arrivals: Widespread bioinvasions in European Seas. Ethology Ecology and Evolution.

Galil, B. S., Boero, F., Campbell, M. L., Carlton, J. T., Cook, E., Fraschetti, S., Gollasch, S., Hewitt, C. L., Jelmert, A., Macpherson, E., Marchini, A., McKenzie, C., Minchin, D., Occhipinti-Ambrogi, A., Ojaveer, H., Olenin, S., Piraino, S. and Ruiz, G. M. 2015a. Letter to the editor: 'Double trouble': the expansion of the Suez Canal and marine bioinvasions in the Mediterranean Sea. Biological Invasions. 17, 973-976.

Galil, B.S., Boero, F., Fraschetti, S., Piraino, S., Campbell, M., Hewitt, C., Carlton, J., Cook, E., Jelmert, A., Macpherson, E., Marchini, A., Occhipinti-Ambrogi, A., McKenzie, C., Minchin, D., Ojaveer, H., Olenin, S. and Ruiz, G. 2015 b. Letter to the editor: Non-indigenous species to the Mediterranean Sea. Limnology and Oceanography Bulletin. 24, 43-45.

Galil, B., Marchini, A., Occhipinti-Ambrogi, A. and Ojaveer, H. 2017. The enlargement of the Suez Canal - Erythraean introductions and management challenges. Management of Biological Invasions. 8(2), 141-152.

Galil, B. S., Mienis, H. K., Hoffman, R. and Goren, M. 2021. Non-indigenous species along the Israeli Mediterranean coast: tally, policy, outlook. Hydrobiologia. 848(9), 2011-2029.

Gallardo, B., Clavero, M., Sánchez, M.I. and Vilà, M. 2016. Global ecological impacts of invasive species in aquatic ecosystems. Global Change Biology. 22(1), pp.151-163.

Gardon, T., Huvet, A., Paul-pont, I., Cassone, A., Koua, M.S., Soyez, C., Jezequel, R., Receveur, J. and Le Moullac, G. 2020. Toxic effects of leachates from plastic pearl-farming gear on embryo-larval development in the pearl oyster Pinctada margaritifera. Water Research. 179. 115890. 
GB Non-native species secretariat, 2020. Recording non-native species. [Online] [Accessed 3 December 2020] Available from: http://www.nonnativespecies.org/index.cfm?sectionid=81

Geburzi, J.C. and McCarthy, M.L. 2018. How Do They Do It? Understanding the Success of Marine Invasive Species In: S. Junblut, V. Liebich and M. Bode, eds. YOUMARES 8 - Oceans Across Boundaries: Learning from each other., pp.109-124.

Gertzen, E., Familiar, O. and Leung, B. 2008. Quantifying invasion pathways: Fish introductions from the aquarium trade. Canadian Journal of Fisheries and Aquatic Sciences. 65, pp.1265-1273.

Giachetti, C.B., Battini, N., Bortolus, A., Tatián, M. and Schwindt, E. 2019. Macropredators as shapers of invaded fouling communities in a cold temperate port. Journal of Experimental Marine Biology and Ecology. 518, p.151177.

Giakoumi, S., Katsanevakis, S., Albano, P.G., Azzurro, E., Cardoso, A.C., Cebrian, E., Deidun, A., Edelist, D., Francour, P., Jimenez, C., Mačić, V., OcchipintiAmbrogi, A., Rilov, G. and Sghaier, Y.R. 2019. Management priorities for marine invasive species. Science of the Total Environment. 688, pp.976-982.

Glasby, T.M. 1999. Effects of shading on subtidal epibiotic assemblages. Journal of Experimental Marine Biology and Ecology. 234(2), pp.275-290.

Glasby, T.M. 2000. Surface composition and orientation interact to affect subtidal epibiota. Journal of Experimental Marine Biology and Ecology. 248(2), pp.177-190.

Glasby, T.M. and Connell, S.D. 2001. Orientation and position of substrata have large effects on epibiotic assemblages. Marine Ecology Progress Series. 214, pp.127-135.

Glasby, T.M., Connell, S.D., Holloway, M.G. and Hewitt, C.L. 2007. Nonindigenous biota on artificial structures: Could habitat creation facilitate biological invasions? Marine Biology. 151(3), pp.887-895. 
Goldberg, C.S., Turner, C.R., Deiner, K., Klymus, K.E., Thomsen, P.F., Murphy, M.A., Spear, S.F., Mckee, A., Oyler-McCance, S.J., Cornman, R.S., Laramie, M.B., Mahon, A.R., Lance, R.F., Pilliod, D.S., Strickler, K.M., Waits, L.P., Fremier, A.K., Takahara, T., Herder, J.E. and Taberlet, P. 2016. Critical considerations for the application of environmental DNA methods to detect aquatic species. Methods in Ecology and Evolution. 7, pp.1299-1307.

Gollasch, S. 2002. The importance of ship hull fouling as a vector of species introductions into the North Sea. Biofouling. 18(2), pp.105-121.

Gonçalves, C., Martins, M., Sobral, P., Costa, P.M. and Costa, M.H. 2019. An assessment of the ability to ingest and excrete microplastics by filter-feeders: A case study with the Mediterranean mussel. Environmental Pollution. 245, pp.600-606.

González-Duarte, M.M., Fernández-Montblanc, T., Bethencourt, M. and Izquierdo, A. 2018. Effects of substrata and environmental conditions on ecological succession on historic shipwrecks. Estuarine, Coastal and Shelf Science. 200, pp.301-310.

Goodenough, A.E. 2010. Are the ecological impacts of alien species misrepresented? A review of the 'native good, alien bad' philosophy. Community Ecology. 11(1), pp.13-21.

Gregory, M.R. 2009. Environmental implications of plastic debris in marine settingsentanglement, ingestion, smothering, hangers-on, hitch-hiking and alien invasions. Philosophical Transactions of the Royal Society B: Biological Sciences. 364, pp.2013-2025.

Gren, I.-M., Isacs, L. and Carlsson, M. 2009. Costs of Alien Invasive Species in Sweden. AMBIO: A Journal of the Human Environment. 38(3), pp.135-140.

Guerra-García, J.M., Ros, M. and Baeza-Rojano, E. 2015. Seasonal fluctuations and dietary analysis of fouling caprellids (Crustacea: Amphipoda) from marinas of southern Spain. Marine Biology Research. 11(7), pp.703-715. 
Gündoğdu, S., Çevik, C. and Karaca, S. 2017. Fouling assemblage of benthic plastic debris collected from Mersin Bay, NE Levantine coast of Turkey. Marine Pollution Bulletin. 124(1), pp.147-154.

Haegerbaeumer, A., Mueller, M., Fueser, H. and Traunspurger, W. 2019. Impacts of Micro- and Nano-Sized Plastic Particles on Benthic Invertebrates: A Literature Review and Gap Analysis. Frontiers in Environmental Science. 7(17), pp.1-33.

Harries, D.B., Cook, E., Donnan, D.W., Mair, J.M., Harrow, S. and Wilson, J.R. 2007. The establishment of the invasive alga Sargassum muticum on the west coast of Scotland: Rapid northwards spread and identification of potential new areas for colonisation. Aquatic Invasions. 2(4), pp.367-377.

Harris, L.G. and Irons, K.P. 1982. Substrate angle and predation as determinants in fouling community succession In: J. Cairns, ed. Artificial substrates. Ann Arbor, MI: Ann Arbor Science Publishers, pp.243-248.

Hayward, P.J. and Ryland, J.S. eds. 2017. Handbook of the Marine Fauna of NorthWest Europe. Oxford: Oxford University Press.

Head, R.M., Davenport, J. and Thomason, J.C. 2004. The effect of depth on the accrual of marine biofilms on glass substrata deployed in the Clyde Sea, Scotland. Biofouling. 20(3), pp.177-180.

Heiman, K.W. and Micheli, F. 2010. Non-native ecosystem engineer alters estuarine communities. Integrative and Comparative Biology. 50(2), pp.226-236.

HELCOM 2013. Joint HELCOM/OSPAR Guidelines on the granting of exemptions under the International Convention for the Control and Management of Ships' Ballast Water and Sediments, Regulation A-4. [Online] Available from: https://helcom.fi/media/documents/Joint-HELCOM_OSPAR-Guidelines.pdf

Henderson, P.A., Plenty, S.J., Newton, L.C. and Bird, D.J. 2012. Evidence for a population collapse of European eel (Anguilla anguilla) in the Bristol Channel. Journal of the Marine Biological Association of the United Kingdom. 92(4), pp.843-851. 
Herbert, R.J.H., Humphreys, J., Davies, C.J., Roberts, C., Fletcher, S. and Crowe, T.P. 2016. Ecological impacts of non-native Pacific oysters (Crassostrea gigas) and management measures for protected areas in Europe. Biodiversity and Conservation. 25, pp.2835-2865.

Hewitt, C.L. and Martin, R.B. 1996. Port surveys for introduced marine speciesbackground considerations and sampling protocols. CRIMP Technical Report No 4, 40 pp.

Hewitt, C.L. and Martin, R.B. 2001. Revised protocols for baseline port surveys for introduced marine species: survey design, sampling protocols and specimen handling. Centre for Research on Introduced Marine Pests. CSIRO Marine Research, Hobart. Technical Report No 22, 46 pp.

Hiscock, K. 2006. A sea squirt (Ascidiella scabra) In: H. Tyler-Walters and K. Hiscock, eds. Marine Life Information Network: Biology and Sensitivity Key Information Reviews [Online]. Plymouth: Marine Biological Association of the United Kingdom. Available from: https://www.marlin.ac.uk/species/detail/1823.

Holmes, S. and Callaway, R. 2020. Design and implementation of two surveys targeted at describing fouling communities and identifying non-native species within active ports. Journal of the Marine Biological Association of the UK. 100, 1191-1204.

Holman, L.E., de Bruyn, M., Creer, S., Carvalho, G., Robidart, J. and Rius, M. (2019) Detection of introduced and resident marine species using environmental DNA metabarcoding of sediment and water. Scientific Reports. 9(1), 11559.

Hooper, D.U., Chapin III, F.S., Ewel, J.J., Hector, A., Inchausti, P., Lavorel, S., Lawton, J.H., Lodge, D.M., Loreau, M., Naeem, S., Schmid, B., Setälä, H., Symstad, A.J., Vandermeer, J. and Wardle, D.A. 2005. Effects of biodiversity on ecosystem functioning: A concensus of current knowledge. Ecological Monographs. 75(1), pp.3-35.

Hulme, P.E. 2009. Trade, transport and trouble: Managing invasive species pathways in an era of globalization. Journal of Applied Ecology. 46(1), pp.10-18. 
Hulme, P.E., Pyšek, P., Jarošík, V., Pergl, J., Schaffner, U. and Vilà, M. 2013. Bias and error in understanding plant invasion impacts. Trends in Ecology and Evolution. 28(4), pp.212-218.

Hurlbut, C.J. 1991. The effects of larval abundance, settlement and juvenile mortality on the depth distribution of a colonial ascidian. Journal of Experimental Marine Biology and Ecology. 150, pp.183-202.

Hurst, H. 2016. Monitoring invasive non-native species in marinas of North West. Report to Natural England. Available from: https://www.livingseasnw.org.uk/sites/default/files/ 201804/NW\%20INNS\%20report\%20HH\%202016\%20FINAL.pdf

IMO, 2011. Guidelines for the control and management of ships' biofouling to minimize the transfer of invasive aquatic species. [Online] [Accessed 11 May 2020] Available from:

https://www.imo.org/en/OurWork/Environment/Pages/Biofouling.aspx

IMO, 2012. Guidance for minimizing the transfer of invasive aquatic species as biofouling (hull fouling) for recreational craft. [Online] [Accessed 11 May 2020] Available from:

https://www.imo.org/en/OurWork/Environment/Pages/Biofouling.aspx

IMO, 2019. International Convention for the Control and Management of Ships'

Ballast Water and Sediments (BWM). [Online] [Accessed 11 May 2020] Available from:

https://www.imo.org/en/About/Conventions/Pages/International-Conventionfor-the-Control-and-Management-of-Ships \%27-Ballast-Water-and-Sediments\%28BWM\%29.aspx

IMO, 2020. Status of Conventions. [Online] [Accessed 11 May 2020] Available from:

https://www.imo.org/en/About/Conventions/Pages/StatusOfConventions.aspx

Inglis, G., Gust, N., Fitridge, I., Floerl, O., Woods, C., Hayden, B. and Fenwick, G. 2005. Opиa Marina Baseline survey for non-indigenous marine species.

Biosecurity New Zealand Technical Paper No: 2005/14. 50pp. 
Jeschke, J.M., Bacher, S., Blackburn, T.M., Dick, J.T.A., Essl, F., Evans, T., Gaertner, M., Hulme, P.E., Kühn, I., Mrugała, A., Pergl, J., Pyšek, P., Rabitsch, W., Ricciardi, A., Richardson, D.M., Sendek, A., Vilà, M., Winter, M. and Kumschick, S. 2014. Defining the impact of non-native species. Conservation Biology. 28(5), pp.1188-1194.

Kakkonen, J.E., Worsfold, T. M., Ashelby, C. W., Taylor, A. and Beaton, K. 2019. The value of regular monitoring and diverse sampling techniques to assess aquatic non-native species: A case study from Orkney. Management of Biological Invasions. 10(1), pp. 46-79.

Kaluza, P., Kölzsch, A., Gastner, M.T. and Blasius, B. 2010. The complex network of global cargo ship movements. Journal of the Royal Society Interface. 7(48), pp.1093-1103.

Kaposi, K.L., Mos, B., Kelaher, B.P. and Dworjanyn, S.A. 2014. Ingestion of microplastic has limited impact on a marine larva. Environmental Science and Technology. 48(3), pp.1638-1645.

Katsanevakis, S., Zenetos, A., Belchior, C. and Cardoso, A.C. 2013. Invading European Seas: Assessing pathways of introduction of marine aliens. Ocean and Coastal Management. 76, pp.64-74.

Katsanevakis, S., Wallentinus, I., Zenetos, A., Leppäkoski, E., Çinar, M.E., Oztürk, B., Grabowski, M., Golani, D. and Cardoso, A.C. 2014. Impacts of invasive alien marine species on ecosystem services and biodiversity: A pan-European review. Aquatic Invasions. 9(4), pp.391-423.

Kauano, R. V., Roper, J.J. and Rocha, R.M. 2017. Small boats as vectors of marine invasion: experimental test of velocity and desiccation as limits. Marine Biology. 164(1), pp.1-10.

Kazmi, S.S.U.H., Xuexi, T., Xu, G., Sikder, M.N.A. and Xu, H. 2020. Vertical variability in taxonomic breadth of biofilm-dwelling ciliates in marine bioassessment surveys. Regional Studies in Marine Science. 38, p.101366. 
Keller, R.P., Drake, J.M., Drew, M.B. and Lodge, D.M. 2011. Linking environmental conditions and ship movements to estimate invasive species transport across the global shipping network. Diversity and Distributions. 17(1), pp.93-102.

Keough, M.J. 1983. Patterns of recruitment of sessile invertebrates in two subtidal habitats. Journal of Experimental Marine Biology and Ecology. 66(3), pp.213245.

Keough, M.J. and Chernoff, H. 1987. Dispersal and Population Variation in the Bryozoan Bugula Neritina. Ecology. 68(1), pp.199-210.

Keough, M.J. and Downes, B.J. 1982. Recruitment of marine invertebrates: the role of active larval choices and early mortality. Oecologia. 54, pp.348-352.

Kettunen, M., Genovesi, P., Gollasch, S., Pagad, S. and Starfinger, U. 2009. Technical support to EU strategy on invasive species (IAS) - Assessment of the impacts of IAS in Europe and the EU (final module report for the European Commission). Institute for European Environmental Policy (IEEP), Brussels, Belgium. 44 pp.

Kraus, R., Ninčević-Gladan, Ž., Auriemma, R., Bastianini, M., Bolognini, L., Cabrini, M., Cara, M., Čalić, M., Campanelli, A., Cvitković, I., Despalatović, M., Dragičević, B., Drakulović, D., Dulčić, J., Flander-Putrle, V., Grati, F., Grego, M., Grilli, F., Jaklin, A., Janeković, I., Kolitari, J., Lipej, L., Magaletti, E., Marini, M., Matić-Skoko, S., Mavrič, B., Mikuš, J., Mozetič, P., OrlandoBonaca, M., Petović, S., Precali, R., Supić, N., Trabucco, B., Travizi, A. and Žuljević, A. 2019. Strategy of port baseline surveys (PBS) in the Adriatic Sea. Marine Pollution Bulletin. 147, pp.47-58.

Lacasa, E., Tsolaki, E., Sbokou, Z., Rodrigo, M.A., Mantzavinos, D. and Diamadopoulos, E. 2013. Electrochemical disinfection of simulated ballast water on conductive diamond electrodes. Chemical Engineering Journal. 223, pp.516-523. 
Lallias, D., Boudry, P., Batista, F.M., Beaumont, A., King, J.W., Turner, J.R. and Lapègue, S. 2015. Invasion genetics of the Pacific oyster Crassostrea gigas in the British Isles inferred from microsatellite and mitochondrial markers. Biological Invasions. 17(9), pp.2581-2595.

Lam, N.W.Y., Huang, R. and Chan, B.K.K. 2009. Variations in intertidal assemblages and zonation patterns between vertical artificial seawalls and natural rocky shores: A case study from Victoria Harbour, Hong Kong. Zoological Studies. 48(2), pp.184-195.

Langhamer, O., Wilhelmsson, D. and Engström, J. 2009. Artificial reef effect and fouling impacts on offshore wave power foundations and buoys - a pilot study. Estuarine, Coastal and Shelf Science. 82(3), pp.426-432.

Leclerc, J.C. and Viard, F. 2018. Habitat formation prevails over predation in influencing fouling communities. Ecology and Evolution. 8(1), pp.477-492.

Leclerc, J.C., Viard, F., González Sepúlveda, E., Díaz, C., Neira Hinojosa, J., Pérez Araneda, K., Silva, F. and Brante, A. 2019. Habitat type drives the distribution of non-indigenous species in fouling communities regardless of associated maritime traffic. Diversity and Distributions. 26(1), pp.62-75.

Lee, S.Y., Jeong, H.J., Seong, K.A., Lim, A.S., Kim, J.H., Lee, K.H., Lee, M.J. and Jang, S.H. 2017. Improved real-time PCR method for quantification of the abundance of all known ribotypes of the ichthyotoxic dinoflagellate Cochlodinium polykrikoides by comparing 4 different preparation methods. Harmful Algae. 63, pp.23-31.

Lehtiniemi, M., Ojaveer, H., David, M., Galil, B., Gollasch, S., McKenzie, C., Minchin, D., Occhipinti-Ambrogi, A., Olenin, S. and Pederson, J. 2015. Dose of truth-Monitoring marine non-indigenous species to serve legislative requirements. Marine Policy. 54, pp.26-35.

Leppäkoski, E. and Olenin, S. 2000. Non-native species and rates of spread: Lessons from the brackish Baltic Sea. Biological Invasions. 2(2), pp.151-163. 
Lezzi, M. and Giangrande, A. 2018. Seasonal and bathymetric effects on macrofouling invertebrates' primary succession in a mediterraenan nonindigenous species hotspot area. Mediterranean Marine Science. 19(3), pp.572-588.

Li, H.X., Orihuela, B., Zhu, M. and Rittschof, D. 2016. Recyclable plastics as substrata for settlement and growth of bryozoans Bugula neritina and barnacles Amphibalanus amphitrite. Environmental Pollution. 218, pp.973-980.

Lincoln, R.J. 1979. British marine amphipoda: gammaridea. London: British Museum Natural History.

Lindeyer, F. and Gittenberger, A. 2011. Ascidians in the succession of marine fouling communities. Aquatic Invasions. 6(4), pp.421-434.

Lins, D.M., de Marco, P., Andrade, A.F.A. and Rocha, R.M. 2018. Predicting global ascidian invasions. Diversity and Distributions. 24(5), pp.692-704.

Liquete, C., Piroddi, C., Drakou, E.G., Gurney, L., Katsanevakis, S., Charef, A. and Egoh, B. 2013. Current Status and Future Prospects for the Assessment of Marine and Coastal Ecosystem Services: A Systematic Review. PLoS ONE. $8(7)$.

Lovell, S.J. and Stone, S.F. 2005. National Center for Environmental Economics The Economic Impacts of Aquatic Invasive Species: A Review of the Literature.

Luan, L., Wang, X., Zheng, H., Liu, L. and Luo, X. 2019. Differential toxicity of functionalized polystyrene microplastics to clams (Meretrix meretrix) at three key development stages of life history. Marine Pollution Bulletin. 139, pp.346354.

Ma, K.C.K. 2020. Rare species detection and benthic recruitment across multiple scales of space and time with implications for early detection of marine invasive species. PhD Thesis. Université Laval, Québec, Canada.

Maceda-Veiga, A., Escribano-Alacid, J., de Sostoa, A. and García-Berthou, E. 2013. The aquarium trade as a potential source of fish introductions in southwestern Europe. Biological Invasions. 15(12), pp.2707-2716. 
Mandić, M., Pestorić, B., Marković, O., Durović, M. and Drakulović, D. 2019. Plankton community of trafficked ports as a baseline reference for Non Indigenous Species arrivals. Case study of the Port of Bar (South Adriatic Sea). Mediterranean Marine Science. 20(4), pp.718-726.

Marins, F.O., Novaes, R.L.M., Rocha, R.M. and Junqueira, A.O.R. 2010. Non indigenous ascidians in port and natural environments in a tropical Brazilian bay. Zoologia. 27(2), pp.213-221.

Maritime \& Coastguard Agency 2020. Ballast Water Management FAQ. [Online] Available from: https://www.gov.uk/government/publications/ballast-watermanagement-faq

Markert, A., Wehrmann, A. and Kröncke, I. 2010. Recently established Crassostreareefs versus native Mytilus-beds: Differences in ecosystem engineering affects the macrofaunal communities (Wadden Sea of Lower Saxony, southern German Bight). Biological Invasions. 12(1), pp.15-32.

Marraffini, M.L., Ashton, G. V., Brown, C.W., Chang, A.L. and Ruiz, G.M. 2017. Settlement plates as monitoring devices for non-indigenous species in marine fouling communities. Management of Biological Invasions. 8(4), pp.559-566.

Marraffini, M.L. and Geller, J.B. 2015. Species richness and interacting factors control invasibility of a marine community. Proceedings of the Royal Society B: Biological Sciences. 282(1812).

Martínez-Gómez, C., León, V.M., Calles, S., Gomáriz-Olcina, M. and Vethaak, A.D. 2017. The adverse effects of virgin microplastics on the fertilization and larval development of sea urchins. Marine Environmental Research. 130, pp.69-76.

Martínez-Laiz, G., Ulman, A., Ros, M. and Marchini, A. 2019. Is recreational boating a potential vector for non-indigenous peracarid crustaceans in the Mediterranean Sea? A combined biological and social approach. Marine Pollution Bulletin. 140, pp.403-415. 
Marty, F., Gueune, H., Malard, E., Sanchez-Amaya, J.M., Sjogren, L., Abbas, B., Quillet, L., van Loosdrecht, M.C.M. and Muyzer, G. 2014. Identification of key factors in Accelerated Low Water Corrosion through experimental simulation of tidal conditions: influence of stimulated indigenous microbiota. Biofouling. 30(3), pp.281-297.

McDonald, J.I., Wilkens, S.L., Stanley, J.A. and Jeffs, A.G. 2014. Vessel generator noise as a settlement cue for marine biofouling species. Biofouling. 30(6), pp.741-749.

McGeoch, M.A., Butchart, S.H.M., Spear, D., Marais, E., Kleynhans, E.J., Symes, A., Chanson, J. and Hoffmann, M. 2010. Global indicators of biological invasion: Species numbers, biodiversity impact and policy responses. Diversity and Distributions. 16(1), pp.95-108.

McGeoch, M.A., Genovesi, P., Bellingham, P.J., Costello, M.J., McGrannachan, C. and Sheppard, A. 2016. Prioritizing species, pathways, and sites to achieve conservation targets for biological invasion. Biological Invasions. 18(2), pp.299-314.

McKenzie, C.H., Matheson, K., Caines, S. and Wells, T. 2016. Surveys for nonindigenous tunicate species in Newfoundland, Canada (2006 - 2014): A first step towards understanding impact and control. Management of Biological Invasions. 7(1), pp.21-32.

Meinesz, A. and Hesse, B. 1991. Introduction of the tropical alga Caulerpa taxifolia and its invasion of the Northwestern Mediterranean. Oceanologica acta. 14(4), pp.415-426.

Mendez, M.M., Schwindt, E. and Bortolus, A. 2015. Differential benthic community response to increased habitat complexity mediated by an invasive barnacle. Aquatic Ecology. 49(4), pp.441-452.

Messinetti, S., Mercurio, S., Scarì, G., Pennati, A. and Pennati, R. 2019. Ingested microscopic plastics translocate from the gut cavity of juveniles of the ascidian Ciona intestinalis. The European Zoological Journal. 86(1), pp.189-195. 
Millennium Ecosystem Assessment 2005. Ecosystems and Human Well-being:

Synthesis. Washington, DC: Island Press. [Online] Available from:

https://www.unenvironment.org/resources/report/ecosystem-and-human-wellbeing-synthesis

Mills, E.L., Leach, J.H., Carlton, J.T. and Secor, C.L. 1993. Exotic Species in the Great Lakes: A History of Biotic Crises and Anthropogenic Introductions. Journal of Great Lakes Research. 19(1), pp.1-54.

Minchin, D., Cook, E.J. and Clark, P.F. 2013. Alien species in British brackish and marine waters. Aquatic Invasions. 8(1), pp.3-19.

Mineur, F., Cook, E., Minchin, D., Bohn, K., MacLeod, A. and Maggs, C. 2012. Changing coasts: Marine aliens and artificial structures. Oceanography and Marine Biology: An Annual Review. 50, pp.189-234.

Miralles, L., Gomez-Agenjo, M., Rayon-Viña, F., Gyraitè, G. and Garcia-Vazquez, E. 2018. Alert calling in port areas: Marine litter as possible secondary dispersal vector for hitchhiking invasive species. Journal for Nature Conservation. 42, pp.12-18.

Molnar, J.L., Gamboa, R.L., Revenga, C. and Spalding, M.D. 2008. Assessing the global threat of invasive species to marine biodiversity. Frontiers in Ecology and the Environment. 6(9), pp.485-492.

Muniz, P., Clemente, J. and Brugnoli, E. 2005. Benthic invasive pests in Uruguay: A new problem or an old one recently perceived? Marine Pollution Bulletin. 50(9), pp.1014-1018.

Muñoz-Colmenero, M., Ardura, A., Clusa, L., Miralles, L., Gower, F., Zaiko, A. and Garcia-Vazquez, E. 2018. New specific molecular marker detects Ficopomatus enigmaticus from water eDNA before positive results of conventional sampling. Journal for Nature Conservation. 43, pp.173-178.

Naylor, E. 1965. Biological effects of a heated effluent in docks at Swansea, S. Wales. Proceedings of the Zoological Society of London. 144, pp.253-268.

Naylor, E. 1957. Brachynotus sexdentatus (Risso), a grapsoid crab new to Britain. Annals and Magazine of Natural History. 10(115), pp.521-523. 
Naylor, R.L., Williams, S.L. and Strong, D.R. 2001. Aquaculture - A gateway for exotic species. Science. 294(5547), pp.1655-1656.

NBN Atlas 2019. National Biodiversity Network (NBN) atlas. Available from: https://nbnatlas.org/.

NBN Atlas 2020. National Biodiversity Network (NBN) atlas. Available from: https://nbnatlas.org/.

Nydam, M. and Stachowicz, J.J. 2007. Predator effects on fouling community development. Marine Ecology Progress Series. 337, pp.93-101.

O’Shaughnessy, K.A., Hawkins, S.J., Yunnie, A.L.E., Hanley, M.E., Lunt, P., Thompson, R.C. and Firth, L.B. 2020. Occurrence and assemblage composition of intertidal non-native species may be influenced by shipping patterns and artificial structures. Marine Pollution Bulletin. 154, p.111082.

Oliviero, M., Tato, T., Schiavo, S., Fernández, V., Manzo, S. and Beiras, R. 2019. Leachates of micronized plastic toys provoke embryotoxic effects upon sea urchin Paracentrotus lividus. Environmental Pollution. 247, pp.706-715.

Olyarnik, S. V., Bracken, M.E.S., Byrnes, J.E., Hughes, A.R., Hultgren, K.M. and Stachowicz, J.J. 2009. Ecological factors affecting community invasibility In: G. Rilov and J. A. Crooks, eds. Biological Invasions in Marine Ecosystems. Berlin Heidelberg: Springe-Verlag, pp.215-238.

Osman, R.W. 1977. The establishment and development of a marine epifaunal community. Ecological Monographs. 47, pp.37-63.

Ostroumov, S.A. 2005. Some aspects of water filtering activity of filter-feeders. Hydrobiologia. 542(1), pp.275-286.

Our life insurance, our natural capital: an EU biodiversity strategy to 2020, COM (2011) 0244, final. [Online] [Accessed 13 May 2020] Available from: https://eur-lex.europa.eu/

Padilla, D.K. 2010. Context-dependent impacts of a non-native ecosystem engineer, the pacific oyster Crassostrea gigas. Integrative and Comparative Biology. 50(2), pp.213-225. 
Padilla, D.K. and Williams, S.L. 2004. Beyond ballast water: aquarium and ornamental trades as sources of invasive species in aquatic ecosystems. Frontiers in Ecology and the Environment. 2(3), pp.131-138.

Painting, S.J., Collingridge, K.A., Durand, D., Grémare, A., Arvanitidis, C. and Bernard, G. 2020. Marine monitoring in Europe: Is it adequate to address environmental threats and pressures? Ocean Science. 16(1), pp.235-252.

Parr, A.C.S., Smith, M.J., Beveridge, C.M., Kerr, A., Cowling, M.J. and Hodgkiess, T. 1998. Optical assessment of a fouling-resistant surface (PHEMA/benzalkonium chloride) after exposure to a marine environment. Advanced Materials for Optics and Electronics. 8(4), pp.187-193.

Peeler, E.J., Oidtmann, B.C., Midtlyng, P.J., Miossec, L. and Gozlan, R.E. 2011. Non-native aquatic animals introductions have driven disease emergence in Europe. Biological Invasions. 13(6), pp.1291-1303.

Pereira, N.N. and Brinati, H.L. 2012. Onshore ballast water treatment: A viable option for major ports. Marine Pollution Bulletin. 64(11), pp.2296-2304.

Perkol-Finkel, S., Ferrario, F., Nicotera, V. and Airoldi, L. 2012. Conservation challenges in urban seascapes: Promoting the growth of threatened species on coastal infrastructures. Journal of Applied Ecology. 49(6), pp.1457-1466.

Pernet, B., Barton, M., Fitzhugh, K., Harris, L.H., Lizárraga, D., Ohl, R. and Whitcraft, C.R. 2016. Establishment of the reef-forming tubeworm Ficopomatus enigmaticus (Fauvel, 1923) (Annelida: Serpulidae) in southern California. BioInvasions Records. 5(1), pp.13-19.

Persson, J., Brett, M.T., Vrede, T. and Ravet, J.L. 2007. Food quantity and quality regulation of trophic transfer between primary producers and a keystone grazer (Daphnia) in pelagic freshwater food webs. Oikos. 116(7), pp.1152-1163.

Peters, K., Sink, K. and Robinson, T.B. 2019. Aliens cruising in: Explaining alien fouling macro-invertebrate species numbers on recreational yachts. Ocean and Coastal Management. 182, p.104986. 
Petrocelli, A., Antolić, B., Bolognini, L., Cecere, E., Cvitković, I., Despalatović, M., Falace, A., Finotto, S., Iveša, L., Mačić, V., Marini, M., Orlando-Bonaca, M., Rubino, F., Trabucco, B. and Žuljević, A. 2019. Port Baseline Biological Surveys and seaweed bioinvasions in port areas: What's the matter in the Adriatic Sea? Marine Pollution Bulletin. 147, pp.98-116.

Pimentel, D., McNair, S., Janecka, J., Wightman, J., Simmonds, C., O’Connell, C., Wong, E., Russel, L., Zern, J., Aquino, T. and Tsomondo, T. 2001. Economic and environmental threats of alien plant, animal, and microbe invasions. Agriculture, Ecosystems and Environment. 84(1), pp.1-20.

Porter, J.S., Spencer Jones, M.E., Kuklinski, P. and Rouse, S. 2015. First records of marine invasive non-native Bryozoa in Norwegian coastal waters from Bergen to Trondheim. BioInvasions Records. 4(3), pp.157-169.

Puth, L.M. and Post, D.M. 2005. Studying invasion: Have we missed the boat? Ecology Letters. 8(7), pp.715-721.

Pyšek, P. and Richardson, D.M. 2010. Invasive Species, Environmental Change and Management, and Health. Annual Review of Environment and Resources. 35(1), pp.25-55.

Qian, P.Y., Lau, S.C.K., Dahms, H.U., Dobretsov, S. and Harder, T. 2007. Marine biofilms as mediators of colonization by marine macroorganisms: Implications for antifouling and aquaculture. Marine Biotechnology. 9(4), pp.399-410.

R Core Team 2017. R: A language and environment for statistical computing. $\mathrm{R}$ Foundation for Statistical Computing, Vienna, Austria. Available from: https://www.r-project.org/.

Rech, S., Borrell Pichs, Y.J. and García-Vazquez, E. 2018. Anthropogenic marine litter composition in coastal areas may be a predictor of potentially invasive rafting fauna. PLoS ONE. 13(1), pp.1-22.

Rech, S., Borrell, Y. and García-Vazquez, E. 2016. Marine litter as a vector for nonnative species: What we need to know. Marine Pollution Bulletin. 113(1-2), pp.40-43. 
Regulation (EU) No 1143/2014 of the European Parliament and of the Council of 22 October 2014 on the prevention and management of the introduction and spread of invasive alien species. [Online] [Accessed 20 December 2019] Available from: http://eur-lex.europa.eu/

Regulation (EU) No. 1143/2014 of 22 October 2014 concerning the prevention and management of the introduction and spread of invasive alien species. [Online] [Accessed 7 August 2020] Available from: http://eur-lex.europa.eu/

Ricciardi, A. and Cohen, J. 2007. The invasiveness of an introduced species does not predict its impact. Biological Invasions. 9(3), pp.309-315.

Ricciardi, A., Hoopes, M.F., Marchetti, M.P. and Lockwood, J.L. 2013. Progress toward understanding the ecological impacts of nonnative species. Ecological Monographs. 83(3), pp.263-282.

Richardson, D.M., Pyšek, P. and Carlton, J.T. 2011. A compendium of essential concepts and terminology in biological invasions In: D. M. Richardson, ed. Fifty years of invasion ecology: The legacy of Charles Elton. Blackwell Publishing Ltd., pp.409-420.

Rilov, G. and Galil, B. 2009. Marine Bioinvasions in the Mediterranean Sea History, Distribution and Ecology In: G. Rilov and J. A. Crooks, eds. Biological Invasions in Marine Ecosystems. Berlin Heidelberg: SpringeVerlag, pp.549-575.

Rius, M., Bourne, S., Hornsby, H.G. and Chapman, M.A. 2015. Applications of nextgeneration sequencing to the study of biological invasions. Current Zoology. 61(3), pp.488-504.

Robinson, C.V., Uren Webster, T.M., Cable, J., James, J. and Consuegra, S. 2018. Simultaneous detection of invasive signal crayfish, endangered white-clawed crayfish and the crayfish plague pathogen using environmental DNA. Biological Conservation. 222, pp.241-252. 
Roche, R.C., Monnington, J.M., Newstead, R.G., Sambrook, K., Griffith, K., Holt, R.H.F. and Jenkins, S.R. 2014. Recreational vessels as a vector for marine nonnatives: developing biosecurity measures and managing risk through an inwater encapsulation system. Hydrobiologia. 750(1), pp.187-199.

Rohde, S., Schupp, P.J., Markert, A. and Wehrmann, A. 2017. Only half of the truth: Managing invasive alien species by rapid assessment. Ocean and Coastal Management. 146, pp.26-35.

Rollins, L.A. 2015. Progress and Challenges in Genetics and Invasion. Current Zoology. 61(3), pp.454-455.

Roman, J. 2006. Diluting the founder effect: Cryptic invasions expand a marine invader's range. Proceedings of the Royal Society B: Biological Sciences. 273(1600), pp.2453-2459.

Ronowicz, M., Kukliński, P., Lock, K., Newman, P.B., Burton, M. and Jones, J. 2014. Temporal and spatial variability of zoobenthos recruitment in a northeast Atlantic marine reserve. Journal of the Marine Biological Association of the United Kingdom. 94(7), pp.1367-1376.

Roy, H. E., Peyton, J., Aldridge, C., Bantock, T., Blackburn, T. M., Britton, R., Clark, P., Cook, E., Dehnen-Schmutz, K., Dines, T., Dobson, M., Edwards, F., Harrower, C., Harvey, M. C., Minchin, D., Noble, D. G., Parrott, D., Pocock, M. J. O., Preston, C. D., Roy, S., Salisbury, A., Schrönrogge, K., Sewell, J., Shaw, R. H., Stebbing, P., Stewart, A. J. and Walker, K. J. 2014. Horizon scanning for invasive alien species with the potential to threaten biodiversity in Great Britain. Global Change Biology. 20(12), 3859-3871.

Roy, H. E., Rabitsch, W., Scalera, R., Stewart, A., Gallardo, B., Genovesi, P., Essl, F., Adriaens, T., Bacher, S., Booy, O., Branquart, E., Brunel, S., Copp, G. H., Dean, H., D’hondt, B., Josefsson, M., Kenis, M., Kettunen, M., Linnamagi, M., Lucy, F., Martinou, A., Moore, N., Nentwig, W., Nieto, A., Pergl, J., Peyton, J., Roques, A., Schindler, S., Schrönrogge, K., Solarz, W., Stebbing, P, D., Trichkova, T., Vanderhoeven, S., van Valkenburg, J. and Zenetos, A. 2018. Developing a framework of minimum standards for the risk assessment of alien species. Journal of Applied Ecology. 55(2), 526-538. 
Ruiz, G.M., Carlton, J.T., Grosholz, E.D. and Hines, A.H. 1997. Global invasions of marine and estuarine habitats by non-indigenous species: mechanisms, extent, and consequences. American Zoologist. 37(6), pp.621-632.

Ruiz, G.M., Fofonoff, P.W., Carlton, J.T., Wonham, M.J. and Hines, A.H. 2000. Invasion of coastal marine communities in North America: Apparent patterns, processes, and biases. Annual Review of Ecology and Systematics. 31, pp.481531.

Ryland, J.S. 1960. The British species of Bugula (Polyzoa). Proceedings of the Zoological Society of London. 134, pp.65-105.

Sakai, A.K., Allendorf, F.W., Holt, J.S., Lodge, M., Molofsky, J., With, K.A., Cabin, R.J., Cohen, J.E., Norman, C., Mccauley, D.E., Neil, P.O., Parker, M., Thompson, J.N. and Weller, S.G. 2001. The population biology of invasive species. Annual Review of Ecology and Systematics. 32, pp.305-332.

Sala, O.E., Chapin, F.S., Armesto, J.J., Berlow, E., Bloomfield, J., Dirzo, R., HuberSanwald, E., Huenneke, L.F., Jackson, R.B., Kinzig, A., Leemans, R., Lodge, D.M., Mooney, H.A., Oesterheld, M., Poff, N.L.R., Sykes, M.T., Walker, B.H., Walker, M. and Wall, D.H. 2000. Global biodiversity scenarios for the year 2100. Science. 287, pp.1770-1774.

Sambrook, K., Holt, R.H.F., Sharp, R., Griffith, K., Roche, R.C., Newstead, R.G., Wyn, G. and Jenkins, S.R. 2014. Capacity, capability and cross-border challenges associated with marine eradication programmes in Europe: The attempted eradication of an invasive non-native ascidian, Didemnum vexillum in Wales, United Kingdom. Marine Policy. 48, pp.51-58.

Sánchez, M.I., Paredes, I., Lebouvier, M. and Green, A.J. 2016. Functional role of native and invasive filter-feeders, and the effect of parasites: Learning from hypersaline ecosystems. PLoS ONE. 11(8), pp.1-19.

Sardain, A., Sardain, E. and Leung, B. 2019. Global forecasts of shipping traffic and biological invasions to 2050. Nature Sustainability. 2, pp.274-282. 
Savini, D., Occhipinti-Ambrogi, A., Marchini, A., Tricarico, E., Gherardi, F., Olenin, S. and Gollasch, S. 2010. The top 27 animal alien species introduced into Europe for aquaculture and related activities. Journal of Applied Ichthyology. 26, pp.1-7.

Schevchenko, O.G., Orlova, T.Y. and Maslennikov, S.I. 2004. Seasonal dynamics of the diatoms of the genus Chaetoceros Ehrenberg in Amursky Bay (Sea of Japan). Russian Journal of Marine Biology. 30, pp.11-19.

Schimanski, K.B., Goldstien, S.J., Hopkins, G.A., Atalah, J. and Floerl, O. 2017. Life history stage and vessel voyage profile can influence shipping-mediated propagule pressure of non-indigenous biofouling species. Biological Invasions. 19(7), pp.2089-2099.

Schimanski, K.B., Piola, R.F., Goldstien, S.J., Floerl, O., Grandison, C., Atalah, J. and Hopkins, G.A. 2016. Factors influencing the en route survivorship and post-voyage growth of a common ship biofouling organism, Bugula neritina. Biofouling. 32(8), pp.969-978.

Schlaepfer, M.A., Sax, D.F. and Olden, J.D. 2011. Revisiting the Potential Conservation Value of Non-Native Species. Conservation Biology. 25(3), pp.428-437.

Schultz, M.P., Bendick, J.A., Holm, E.R. and Hertel, W.M. 2011. Economic impact of biofouling on a naval surface ship. Biofouling. 27(1).

Seebens, H., Gastner, M.T. and Blasius, B. 2013. The risk of marine bioinvasion caused by global shipping. Ecology Letters. 16(6), pp.782-790.

Seebens, H., Schwartz, N., Schupp, P.J. and Blasius, B. 2016. Predicting the spread of marine species introduced by global shipping. Proceedings of the National Academy of Sciences of the United States of America. 113(20), pp.5646-5651.

Sella, I. and Perkol-Finkel, S. 2015. Blue is the new green - Ecological enhancement of concrete based coastal and marine infrastructure. Ecological Engineering. 84, pp.260-272.

Shenkar, N., Shmuel, Y. and Huchon, D. 2018. The invasive ascidian Ciona robusta recorded from a Red Sea marina. Marine Biodiversity. 48(4), pp.2211-2214. 
Shiganova, T.A. 1998. Invasion of the Black Sea by the ctenophore Mnemiopsis leidyi and recent changes in pelagic community structure. Fisheries Oceanography. 7(3-4), pp.305-310.

Shucksmith, R., Cook, E.J., Hughes, D.J. and Burrows, M.T. 2009. Competition between the non-native amphipod Caprella mutica and two native species of caprellids Pseudoprotella phasma and Caprella linearis. Journal of the Marine Biological Association of the United Kingdom. 89(6), pp.1125-1132.

Silva, P.P.G.E., Nobre, C.R., Resaffe, P., Pereira, C.D.S. and Gusmão, F. 2016. Leachate from microplastics impairs larval development in brown mussels. Water Research. 106, pp.364-370.

Simberloff, D., Martin, J.L., Genovesi, P., Maris, V., Wardle, D.A., Aronson, J., Courchamp, F., Galil, B., García-Berthou, E., Pascal, M., Pyšek, P., Sousa, R., Tabacchi, E. and Vilà, M. 2013. Impacts of biological invasions: What's what and the way forward. Trends in Ecology and Evolution. 28(1), pp.58-66.

Skovhus, T.L., Ramsing, N.B., Holmström, C., Kjelleberg, S. and Dahllöf, I. 2004. Real-Time Quantitative PCR for Assessment of Abundance of Pseudoalteromonas Species in Marine Samples. Applied and Environmental Microbiology. 70(4), pp.2373-2382.

Smith, M., Bardiau, M., Brennan, R., Burgess, H., Caplin, J., Ray, S. and Urios, T. 2019. Accelerated low water corrosion: the microbial sulfur cycle in microcosm. Materials Degradation. 3(1).

Sorte, C.J.B, Williams, S.L., Carlton, J.T. 2010. Marine range shifts and species introductions: comparative spread rates and community impacts. Global Ecology and Biogeography. 19(3), pp. 303-316.

South, P., Floerl, O. and Jeffs, A. 2019. The role of biofouling development in the loss of seed mussels in aquaculture. Biofouling. 35(2), pp.259-272.

Stein, R.A., De Vries, D.R. and Dettmers, J.M. 1995. Food-web regulation by a planktivore: exploring the generality of the trophic cascade hypothesis. Canadian Journal of Fisheries and Aquatic Sciences. 52(11), pp.2518-2526. 
Streftaris, N. and Zenetos, A. 2006. Alien Marine Species in the Mediterranean - the 100 'Worst Invasives' and their Impact. Mediterranean Marine Science. 7(1), pp.87-118.

Streftaris, N., Zenetos, A. and Papathanassiou, E. 2005. Globalisation in marine ecosystems: The story of non-indigenous marine species across European seas. Oceanography and Marine Biology. 43, pp.419-453.

Sueiro, M.C., Bortolus, A. and Schwindt, E. 2011. Habitat complexity and community composition: Relationships between different ecosystem engineers and the associated macroinvertebrate assemblages. Helgoland Marine Research.65(4), pp.467-477.

Sutherland, J.P. and Karlson, R.H. 1977. Development and Stability of the Fouling Community at Beaufort, North Carolina. Ecological Monographs. 47(4), pp.425-446.

Svane, I. and Gröndahl, F. 1988. Recruitment and development of epibioses on artificial and cleared substrata at two site in Gullmarsfjorden on the Swedish west coast. Ophelia. 29, pp.25-41.

Tallec, K., Huvet, A., Di, C., Gonz, C., Lambert, C., Petton, B., Le, N., Berchel, M., Soudant, P. and Paul-pont, I. 2018. Nanoplastics impaired oyster free living stages, gametes and embryos. Environmental Pollution. 242, pp.1226-1235.

Thiel, M. 1998. Extended parental care in marine amphipods. I. Juvenile survival without parents. Journal of Experimental Marine Biology and Ecology. 227(2), pp.187-201.

Tøttrup, A.P., Chan, B.K.K., Koskinen, H. and Høeg, J.T. 2010. 'Flying barnacles': implications for the spread of non-indigenous species. Biofouling. 26(5), pp.577-582. 
Travizi, A., Balković, I., Bacci, T., Bertasi, F., Cuicchi, C., Flander-Putrle, V., Grati, F., Grossi, L., Jaklin, A., Lipej, L., Mavrič, B., Mikac, B., Marusso, V., Montagnini, L., Nerlović, V., Penna, M., Salvalaggio, V., Santelli, A., Scirocco, T., Spagnolo, A., Trabucco, B. and Vani, D. 2019. Macrozoobenthos in the Adriatic Sea ports: Soft-bottom communities with an overview of nonindigenous species. Marine Pollution Bulletin. 147, pp.159-170.

Tricarico, E., Borrell, Y.J., García-Vázquez, E., Rico, J.M., Rech, S., Scapini, F., Johović, I., Rodríguez-Ezpeleta, N., Basurko, O.C., Rey, A., Gough, P., Aquiloni, L., Sposimo, P., Inghilesi, A.F., Haubrock, P., Delgado, J.F., Skukan, R., Hall, D., Marsh-Smith, S., Kilbey, D., Monteoliva, A.P., Muha, T.P., Rodríguez-Rey, M., Rolla, M., Rehwald, H.K., De Leaniz, C.G. and Consuegra, S. 2017. Developing innovative methods to face aquatic invasions in Europe: The Aquainvad-ED project. Management of Biological Invasions. 8(3), pp.403-408.

Tsiamis, K., Azzurro, E., Bariche, M., Çinar, M. E., Crocetta, F., De Clerck, O., Galil, B., Gómez, F., Hoffman, R., Jensen, K. R., Kamburska, L., Langeneck, J., Langer, M. R., Levitt-Barmats, Y., Lezzi, M., Marchini, A., OcchipintiAmbrogi, A., Ojaveer, H., Piraino, S., Shenkar, N., Yankova, M., Zenetos, A., Žuljević, A. and Cardoso, A. C. 2020. Aquatic Conservation: Marine and Freshwater Ecosystems. 30(4), 794-845.

Tsolaki, E. and Diamadopoulos, E. 2010. Technologies for ballast water treatment: A review. Journal of Chemical Technology and Biotechnology. 85(1), pp.19-32.

Tyrrell, M.C. and Byers, J.E. 2007. Do artificial substrates favor nonindigenous fouling species over native species? Journal of Experimental Marine Biology and Ecology. 342, pp.54-60.

UK Government 2015. The Great Britain Invasive Non-native Species Strategy. [Online] Available from: https://www.gov.uk/government/publications/thegreat-britain-invasive-non-native-species-strategy 
Ulman, A., Ferrario, J., Forcada, A., Seebens, H., Arvanitidis, C., OcchipintiAmbrogi, A. and Marchini, A. 2019. Alien species spreading via biofouling on recreational vessels in the Mediterranean Sea. Journal of Applied Ecology. 56(12), pp.2620-2629.

UN, 1992. Convention on Biological Diversity. [Online] [Accessed 14 May 2020] Available from: https://www.cbd.int/convention/text/

UNCED, 1992. United Nations Conference on Environment and Development, Agenda 21. [Online] [Accessed 14 May 2020] Available from: https://sustainabledevelopment.un.org/outcomedocuments/agenda21

UNEP, 2011. Annual Report 2011. [Online] [Accessed 14 May 2020] Available from: https://www.unenvironment.org/resources/annual-report/unep-2011annual-report

Viard, F., Ellien, C. and Dupont, L. 2006. Dispersal ability and invasion success of Crepidula fornicata in a single gulf: Insights from genetic markers and larvaldispersal model. Helgoland Marine Research. 60(2), pp.144-152.

Vidjak, O., Bojanić, N., de Olazabal, A., Benzi, M., Brautović, I., Camatti, E., Hure, M., Lipej, L., Lučić, D., Pansera, M., Pećarević, M., Pestorić, B., Pigozzi, S. and Tirelli, V. 2019. Zooplankton in Adriatic port environments: Indigenous communities and non-indigenous species. Marine Pollution Bulletin. 147, pp.133-149.

Vieira, E.A., Flores, A.A.V. and Dias, G.M. 2018. Persistence and space preemption explain species-specific founder effects on the organization of marine sessile communities. Ecology and Evolution. 8(6), pp.3430-3442.

Vilà, M. and Hulme, P. (eds.). 2017. Impact of Biological Invasions on Ecosystem Services. Springer International Publishing.

Vitule, J.R.S., Freire, C.A. and Simberloff, D. 2009. Introduction of non-native freshwater fish can certainly be bad. Fish and Fisheries. 10(1), pp.98-108. 
Walsh, J.R., Carpenter, S.R. and Van Der Zanden, M.J. 2016. Invasive species triggers a massive loss of ecosystem services through a trophic cascade. Proceedings of the National Academy of Sciences of the United States of America. 113(15), pp.4081-4085.

Welsh Government 2017. Marine Invasive Non-native Species Priority Monitoring and Surveillance List for Wales. [Online] Available from: https://gov.wales/sites/default/files/publications/2018-02/invasive-aquaticspecies-priority-marine-species.pdf

Williams, F.E., Eschen, R., Harris, A., Djeddour, D.H., Pratt, C.F., Shaw, R.S., Varia, S., Lamontagne-Godwin, J.D., Thomas, S.E. and Murphy, S.T. 2010. The economic cost of invasive non-native species on Great Britain. Wallingford, UK. [Online] Available from: https://www.cabi.org/VetMedResource/ebook/20123122024

Williams, S.L., Davidson, I.C., Pasari, J.R., Ashton, G. V, Carlton, J.T., Crafton, R.E., Fontana, R.E., Grosholz, E.D., Miller, A.W., Ruiz, G.M. and Zabin, C.J. 2013. Managing Multiple Vectors for Marine Invasions in an Increasingly Connected World. BioScience. 63(12), pp.952-966.

Willis, K.J., Cook, E.J., Lozano-Fernandez, M. and Takeuchi, I. 2004. First record of the alien caprellid amphipod, Caprella mutica, for the UK. Journal of the Marine Biological Association of the United Kingdom. 84(5), pp.1027-1028.

Wonham, M.J., O’Connor, M. and Harley, C.D.G. 2005. Positive effects of a dominant invader on introduced and native mudflat species. Marine Ecology Progress Series. 289, pp.109-116.

Wrange, A.L., Charrier, G., Thonig, A., Rosenblad, M.A., Blomberg, A., Havenhand, J.N., Jonsson, P.R. and André, C. 2016. The story of a hitchhiker: Population genetic patterns in the invasive barnacle Balanus (Amphibalanus) improvisus Darwin 1854. PLoS ONE. 11(1), pp.1-28.

Wright, D.A., Gensemer, R.W., Mitchelmore, C.L., Stubblefield, W.A., van Genderen, E., Dawson, R., Orano-Dawson, C.E., Bearr, J.S., Mueller, R.A. and Cooper, W.J. 2010. Shipboard trials of an ozone-based ballast water treatment system. Marine Pollution Bulletin. 60(9), pp.1571-1583. 
Yee, A., Mackie, J. and Pernet, B. 2019. The distribution and unexpected genetic diversity of the non-indigenous annelid Ficopomatus enigmaticus in California. Aquatic Invasions. 14(2), pp.250-266. 